Daniel Akira Maêda

Modelagem da estampagem profunda de chapas metálicas via o método dos elementos finitos associado ao critério de escoamento não-quadrático de Hill 

Daniel Akira Maêda

\section{Modelagem da estampagem profunda de chapas metálicas via o método dos elementos finitos associado ao critério de escoamento não-quadrático de Hill}

Dissertação apresentada à Escola de Engenharia de São Carlos da Universidade de São Paulo para a obtenção do título de Mestre em Engenharia Mecânica.

Área de Concentração: Projeto Mecânico.

Orientador:

Prof. Dr.-ing Carlos Henrique Daros

São Carlos - SP

Abril / 2009 
AUTORIZO A REPRODUÇÃO E DIVULGAÇÃO TOTAL OU PARCIAL DESTE TRABALHO, POR QUALQUER MEIO CONVENCIONAL OU ELETRÔNICO, PARA FINS DE ESTUDO E PESQUISA, DESDE QUE CITADA A FONTE.

Ficha catalográfica preparada pela Seção de Tratamento da Informação do Serviço de Biblioteca - EESC/USP

Maeda, Daniel Akira

M184m

Modelagem da estampagem profunda de chapas metálicas via o método dos elementos finitos associado ao critério de escoamento não-quadrático de Hill / Daniel Akira Maeda ; orientador Carlos Henrique Daros. -- São Carlos, 2009.

Dissertação (Mestrado-Programa de Pós-Graduação em Engenharia Mecânica e Área de Concentração Projeto Mecânico) -- Escola de Engenharia de São Carlos da Universidade de São Paulo, 2009.

1. Método dos elementos finitos. 2. Critério de escoamento não quadrático. 3. Conformação de chapas metálicas. 4. Estampagem profunda. I. Título. 
Aos meus pais Hélio e Leiko. 


\section{Agradecimentos}

Ao Professor Carlos Henrique Daros pela dedicação, orientação e amizade

Aos Professores Jaime, Gilmar, Renato e Luciana pelo todo apoio dado.

Aos amigos do Laboratório de Mecânica Computacional e do Laboratório de Engenharia de Precisão, Alexandre, Mariano, Toddy, João Paulo e Zé Antônio.

Aos funcionários da secretaria de Pós-graduação, Ana Paula e Iuri, pela eficiência na resolução de questões burocráticas.

À empresa HPB pelo apoio dado para a finalização deste trabalho.

Ao CNPq, Conselho Nacional de Desenvolvimento Científico e Tecnológico, pela bolsa de estudo sem a qual seria impossível a realização deste trabalho. 


\section{Resumo}

MAEDA, D. A.(2009). Modelagem da estampagem profunda de chapas metálicas via o método dos elementos finitos associado ao critério de escoamento não-quadrático de Hill. Dissertação (Mestrado) - Escola de Engenharia de São Carlos, Universidade de São Paulo.

Os processos de conformação de chapas metálicas são largamente usados na fabricação de produtos em diversas áreas, desde partes aeronáuticas a utensílios domésticos, devido a sua alta produtividade, confiabilidade e baixo custo de produção. Para se atingir tais qualidades, o desenvolvimento de um produto conformado deve levar em consideração os fatores metalúrgicos da chapa a ser usada. As chapas são geralmente produzidas por laminação a frio, o que as levam ter propriedades mecânicas distintas em relação à direção de laminação. Para modelar esta anisotropia, vários critérios de escoamento foram propostos. A fim de verificar a influência do critério de escoamento na distribuição de deformação no produto conformado, este trabalho teve como objetivo implementar os critérios de Hill (1979) e de Barlat et al. (1993) em um programa acadêmico que modela o processo de conformação de chapas via o Método dos Elementos Finitos. O critério de Hill (1979) foi implementado e apresentou bons resultados, em acordo com a literatura. O critério de Barlat et al. (1993) foi deduzido em sua formulação para elementos finitos, embora não implementado. Com o critério de Hill (1979) foi possível analisar a distribuição da deformação para várias superfícies de escoamento, alterando-se apenas o valor de um parâmetro da função de escoamento.

Palavras-chave: Método dos elementos finitos, critério de escoamento não quadrático, conformação de chapas metálicas, estampagem profunda. 


\section{Abstract}

MAEDA, D. A.(2009). Modelling of sheet metal deep drawing via finite element method associated with Hill's non-quadratic yield criterion. Dissertation (M. Sc.) - Escola de Engenharia de São Carlos, Universidade de São Paulo.

The sheet metal forming processes are widely used in the manufacturing of products in several areas, from aviation to household utensils, due to its high productivity, reliability and low cost of production. To achieve these qualities, the design of a product made by sheet metal forming should take into consideration the metallurgical factors of the blank to be used. The blank is usually cold-rolled, which leads to different mechanical properties in the rolled and transverse directions. To take into account this anisotropy, several yield criteria were proposed. To check the influence of the yield criterion in the strain distribution of the final product, this study focused on the implementation of the yield criteria of Hill (1979) and Barlat et al. (1993) in an academic computer program that modeled the sheet metal forming process by the FInite Element Method. The Hill (1979) criterion was implemented and had good results, in accordance with the literature. The Barlat et al. (1993) criterion was formulated for a FInite Element Method analysis, though not implemented. By using Hill's non-quadratic yield criterion it was possible to analyze the strain distribution for various yield surfaces, changing only the value of one parameter of the yield function.

Keywords: FInite element method, non-quadratic yield criterion, sheet metal forming, deep-drawing. 


\section{Lista de Figuras}

1 Estampagem profunda: (A) Primeira operação; (B)Segunda operação; (C) Extração; Em estampagem profunda, o blank é pressionado contra a matriz, através do punção, rebaixando a superfície sem alterar sensivelmente a espessura original (Kobayashi et al. (1989)). . . . . . . . . . . $\quad$ p. 2

2 Defeitos observados em estampagem de peças ocas (Kobayashi et al.

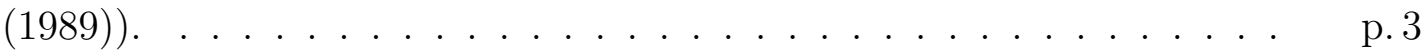

3 Diagramas de tensão: (a) Piola-Kirchhoff; (b) Cauchy (tensão verdadeira) (Kobayashi et al. (1989)). . . . . . . . . . . . . . . p. 12

4 Ilustração Geométrica da regra associada de fluxo (Chen e Han (1988)).

5 Convexidade da superfície de campo e normalidade do fluxo plástico.

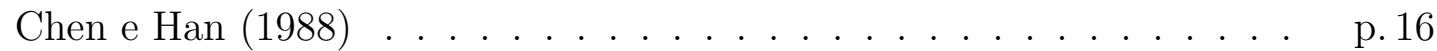

6 Representação geometrica de estado plástico de tensão no espaço (Kobayashi et al. (1989)). . . . . . . . . . . . . . . . p. 20

7 "Locci" do escoamento no plano p para o critério de distorção de energia e tensão cisalhante (Kobayashi et al. (1989)). . . . . . . . . . . . . p. 21

$8 \quad$ Curva teórica de tensão-deformação. Toh (1983) . . . . . . . . . . p. 33

9 Um elemento de chapa mostrando: a) estado inicial com uma grade de círculos e quadrados marcados, b) estado deformado com os círculos deformados em elipses com os eixos $d_{1}$ e $d_{2}$ e c) as trações aplicadas (Marciniak et al. (2002)). . . . . . . . . . . . . . . . 
10 a) Estampagem profunda de um copo circular, b) seção do copo mostrando a localização das deformações medidas e c) deformações plotadas para dois estágios do processo de estampagem (Marciniak et al. (2002)).

11 O diagrama de deformação mostrando os diferentes modos de deformação correspondendo a diferentes razões de deformação (Marciniak et al. (2002)). p. 40

12 Estiramento equibiaxial no centro da chapa (Marciniak et al. (2002)).

13 Deformação no estado plano de deformação em uma parede de uma chapa comprida (Marciniak et al. (2002)). . . . . . . . . . . . p. 41

14 Tração uniaxial em um furo expandido (Marciniak et al. (2002)). . . . . p. 42

15 Repuxo ou cisalhamento puro na flange de uma estampagem profunda de copo cilíndrico (Marciniak et al. (2002)). . . . . . . . . . . . . . p. p. 42

16 Compressão uniaxial na borda da estampagem profunda (Marciniak et al.

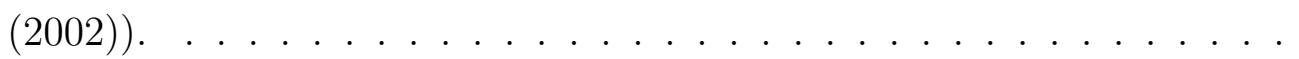

O processo mostrado na figura 10 representado no espaço de tensões (a elipse de escoamento representada em linha tracejada) (Marciniak et al.

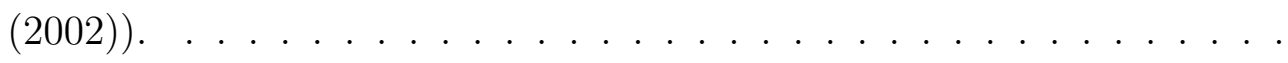

18 Variáveis do dobramento (Marciniak e Duncan (1992)) . . . . . . . . p. 46

19 Conformação de um blank circular (Marciniak e Duncan (1992)). . . .

20 Superfície de revolução gerada pela revolução da curva $C$ (Marciniak e Duncan (1992)). . . . . . . . . . . . . . . . . p 48

21 Conformando uma chapa fina com pressão de fluido (Marciniak e Duncan (1992)) 
23 (a)Força de atrito na interface punção-chapa; (b) influencia do atrito na deformação; (c) ruptura usualmente próxima a região $B$ (Marciniak e Duncan $(1992)) \ldots \ldots \ldots \ldots \ldots \ldots \ldots \ldots \ldots \ldots \ldots \ldots \ldots \ldots$

24 Geometria e forças na estampagem profunda de um copo cilíndrico (Marciniak e Duncan (1992)). . . . . . . . . . . . . . p. 54

25 (a) Regiões de estiramento e estampagem; (b) força máxima Marciniak

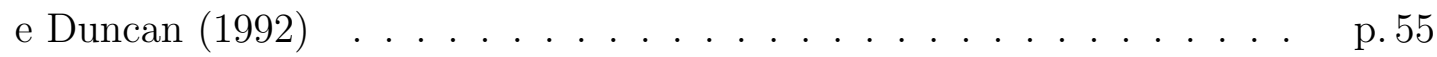

26 (a) Forças na aba; (b) coordenadas em um espaço oblíquo; (c) deformações no espaço oblíquo (Marciniak e Duncan (1992)). . . . . . . . . . p. p.56

27 Espessura na parede do copo (Marciniak e Duncan (1992)). . . . . . . . p p.56

28 Forças de atrito no prensa-chapas (Marciniak e Duncan (1992)) . . . . p. p.58

29 Efeito do atrito em uma tira puxada em um cilindro (Marciniak e Duncan

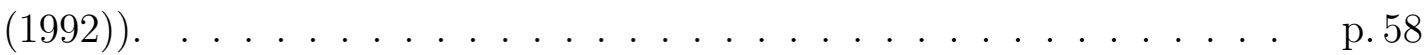

30 Força no punção (Marciniak e Duncan (1992)). . . . . . . . . . . . . p. 59

31 (a)Pressão de contato no raio do punção; (b) distribuição da tensão (Marciniak e Duncan (1992)). . . . . . . . . . . . p. 60

32 Afinamento no raio do punção (Marciniak e Duncan (1992)). . . . . . . p. 61

33 Atrito na parede do punção (Marciniak e Duncan (1992)). . . . . . . . p. p. 62

34 (a) Variação na força $T_{\phi b}$, de estiramento no fundo, com a profundidade $h_{b}$ da região estirada (Marciniak e Duncan (1992)). . . . . . . . . . . . p. p.63

35 Cinemática da deformação do elemento de chapa nos vários estágios

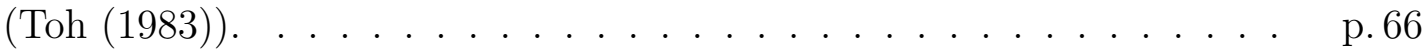

36 Coordenadas locais do elemento triangular com os deslocamentos nodais u, ve w $($ Toh $(1983)) . \ldots \ldots \ldots \ldots \ldots$ p. . . . . . . . . . . . . . . . . 
37 Sistemas de coordenadas Cartesianas global e local (Toh (1983)). . . . $\quad$ p.76

38 Vista esquemática dos requisitos geometricos para o nó em contato com o punção $($ Toh $(1983)) \ldots \ldots \ldots \ldots$ p. . . . . . . . . . . . . . . . .

39 Condição de contorno para o nó em contato com o punção (Toh (1983)). p. 83

40 Condições de contorno para o estiramento (Toh (1983)). . . . . . . . p p. 84

41 Condições de contorno para a parte da flange na estampagem profunda. Área hachurada indica a região da flange (Toh (1983)). . . . . . . . . . . p. 85

42 Vista esquemática da estampagem profunda com copo quadrado. Toh (1983) p. 86

43 Vista das condições de contorno em uma chapa quadrada antes da deformação. Toh $(1983) \ldots \ldots$. . . . . . . . . . . . p. 87

44 Vista esquemática do (a) estiramento e (b) estampagem profunda. (Toh

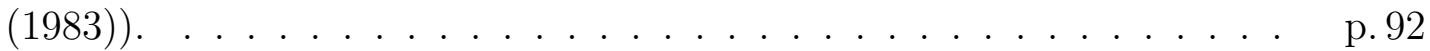

45 Fluxograma do programa SHEET (Toh (1983)). . . . . . . . . . . p. 95

$46 \quad$ Malha usada na estampagem profunda com punção hemisférico. . . . . p.97

47 Comparação entre os resultados obtidos por Toh (1983) e a presente dissertação, para a distribuição da deformação na espessura para vários

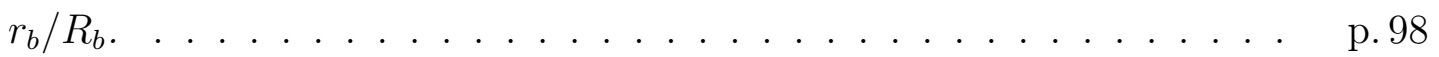

48 Comparação entre os resultados obtidos por Toh (1983) e a presente dissertação, para a força no punção. . . . . . . . . . . . . . . . . p. p. 99

49 Malha usada na modelagem. . . . . . . . . . . . . . . . p. 99

50 Deformação na espessura na diagonal, comparação dos resultado obtidos com os de Toh (1983). . . . . . . . . . . . . . . . . . p. 100

51 Deformação na espessura em uma malha não otimizada. . . . . . . . . p. 100 
52 Deformação na espessura no eixo $x$, comparação dos resultados obtidos com os de Toh (1983). . . . . . . . . . . . . . . . . . . p. 101

53 Distribuição da tensão no eixo $x$ (dividido pela constante $K$, equação 3.87), comparação dos resultados obtidos com os de Toh (1983). . . . . . . p. 101

54 Distribuição da tensão na diagonal (dividido pela constante $K$, equação 3.87), comparação dos resultados obtidos com os de Toh (1983). . . . . . p p. 102

55 Malha deformada na profundidade final de $20,2 \mathrm{~mm} . \ldots \ldots$. . . . . 102

56 Comparação entre os resultados obtidos pelo programa SHEET original e o SHEET modificado. . . . . . . . . . . . . . . . . . . p. 104

57 Malha usada no estiramento de blank circular . . . . . . . . . . p. 105

58 Comparação entre os resultados obtidos numericamente e os resultados

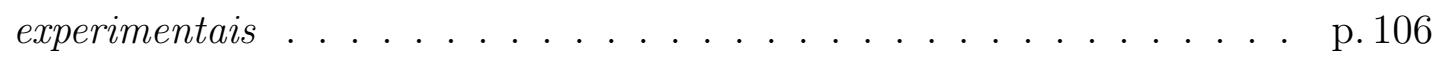

59 Superfícies de escoamento para a tensão plana (linhas contínuas) e superfície da taxa de deformação associada (linhas descontínuas) (Lian et al. (1989)) . . . . . . . . . . . . . . . . . . . 107

60 Superfície de escoamento para tensão plana, $r=R=0,5$ e $m$ é o mesmo que $M$ (Parmar e Mellor (1978)). . . . . . . . . . . . . . . p. 108

61 Malha usada na estampagem profunda . . . . . . . . . . . p. 111

62 Deformação na espessura na diagonal . . . . . . . . . . . . . . p. 111

63 Distribuição da deformação na espessura na peça final: a) $M=1,6$ e

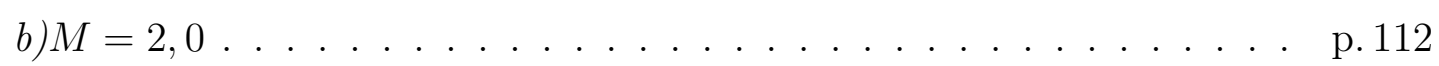

64 Distribuição da deformação na espessura: a) $M=1,6$ e b) $M=2,0 \quad \ldots \quad$ p. 113

65 Parâmetros mensuradas Danckert (1995). . . . . . . . . . . p. 116

66 Deformação na espessura na diagonal. . . . . . . . . . . . . . p. 116 
67 Deformação na espessura na diagonal. . . . . . . . . . . . . . . . p. 117

68 Deformações principais na diagonal. . . . . . . . . . . . . . . p. 117

69 Distribuição da deformação na espessura (profundidade $15 \mathrm{~mm}$ ): a) $M=$

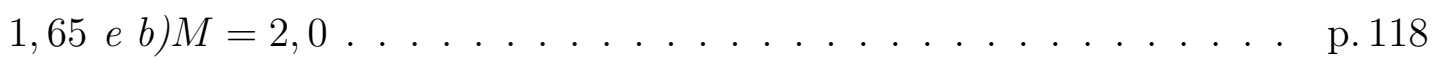

70 Distribuição da deformação na espessura na peça final (profundidade 15 mm): a) $M=1,65$ e b) $M=2,0 \ldots \ldots \ldots$. . . . . . . . . . 119

71 Formação de orelhas (profundidade $15 \mathrm{~mm}$ ): a) $M=1,65$ e b) $M=2,0 \quad$ p. 120

72 Distribuição da deformação na espessura (profundidade $40 \mathrm{~mm}$ ): a) $M=$

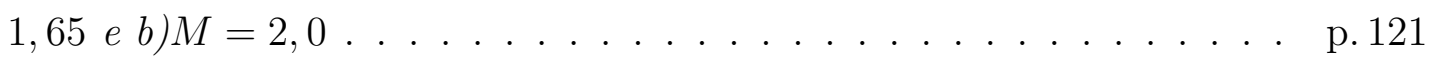

73 Distribuição da deformação na espessura na peça final (profundidade 40 mm): a) $M=1,65$ e b) $M=2,0 \ldots \ldots \ldots$. . . . . . . . 122

74 Formação de orelhas (profundidade $40 \mathrm{~mm}$ ): a) $M=1,65$ e b) $M=2,0 \quad$ p. 123 


\section{Lista de Tabelas}

1 Tabela com deslocamentos em DD. Valores experimentais por Danckert

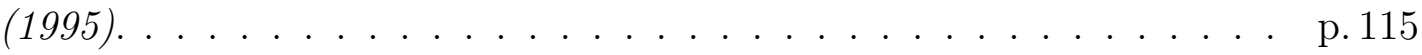

2 Tabela com deslocamentos em DX, Valores experimentais por Danckert

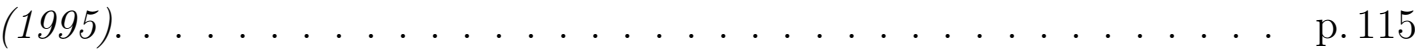




\section{Sumário}

1 Introdução $\quad$ p. 1

1.1 Escopo de Estudo . . . . . . . . . . . . . . . . . p. 1

1.2 Objetivos da Dissertação . . . . . . . . . . . . . . . . p. 3

1.3 Conteúdo desta Dissertação . . . . . . . . . . . . . . p. 4

2 Revisão Bibliográfica p. 5

3 Elementos de Plasticidade $\quad$ p. 11

3.1 Introdução . . . . . . . . . . . . . . . . . . p. 11

3.2 Regra do Fluxo e Potencial Plástico . . . . . . . . . . . . . . p. 14

3.3 Critério de Escoamento . . . . . . . . . . . . . p. 18

3.3.1 Equações de Prandtl-Reuss e Levy-Mises . . . . . . . . . . . . . p. 20

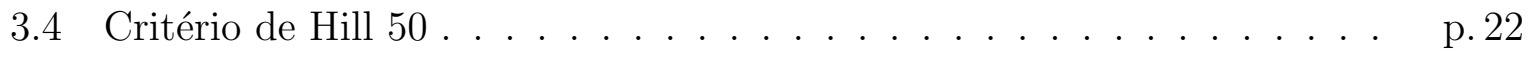

3.5 Critério de Hill $79 \ldots \ldots \ldots \ldots \ldots$ p. . . . . . . . . . . . . . . . . .

3.6 Critério de Barlat . . . . . . . . . . . . . . . . . . p. 29

3.7 Equação Constitutiva para a Curva Tensão Deformação de Materiais Rígido-plásticos . . . . . . . . . . . . . . . p. 32

3.8 Zona Rígida . . . . . . . . . . . . . . . . . . . . p. 34

4 Mecânica da Deformação de chapas $\quad$ p. 37 
4.1 Deformação da Chapa no Estado Plano de Tensão . . . . . . . . . . . . p. 37

4.1 .1 Distribuição de Deformações . . . . . . . . . . . . . . . . . . p. 37

4.1.2 Diagrama de Deformação . . . . . . . . . . . . . . . p. p. 38

4.1 .3 Modos de Deformação . . . . . . . . . . . . . . . . . . . . p. 39

4.1 .4 Diagrama de tensão . . . . . . . . . . . . . . . . . . . . p. 42

4.2 Estudo do Dobramento . . . . . . . . . . . . . . p. 45

4.3 Estudo do Estiramento . . . . . . . . . . . . . . . . p. p 47

4.3.1 Estiramento de um Diafragma Circular . . . . . . . . . . p. 47

4.3.2 Condições de Equilíbrio . . . . . . . . . . . . . . . . p. 50

4.3.3 Estiramento com Punção Hemisférico Rígido . . . . . . . . . . . p. p.51

4.3 .4 Efeito do Atrito . . . . . . . . . . . . . . p. 52

4.4 Estampagem Profunda . . . . . . . . . . . . . . . . . . . . p. 53

4.4 .1 Deformação da Espessura na Aba . . . . . . . . . . . . . . p. 54

4.4 .2 Espessura da Parede . . . . . . . . . . . . . . . . p. 55

4.4.3 Efeito do Atrito do Prensa-chapas . . . . . . . . . . p. 57

4.4.4 Atrito no Raio da Matriz . . . . . . . . . . . . p. 57

4.4.5 Efeito do Contato de Pressão no Punção Cilíndrico . . . . . . p. p.59

4.4.6 Afinamento no Raio do Punção . . . . . . . . . . . . . . . . p. 60

4.4 .7 Atrito no Punção . . . . . . . . . . . . . . . . p. 61

5 Modelagem do Processo de Estampagem pelo Método dos Elemen$\begin{array}{ll}\text { tos Finitos p. } 65 & \text { p }\end{array}$

5.1 Abordagem Variacional . . . . . . . . . . . . . p. 65 
5.2 Cinemática da Deformação . . . . . . . . . . . . . . p. 66

5.3 Discretização . . . . . . . . . . . . . . . . . . . . . . . . . . . p. 69

5.4 Translação de Coordenadas . . . . . . . . . . . . . . p. 76

5.5 Método de Solução . . . . . . . . . . . . . . . . . . . . . p. 78

5.6 Condições de Contorno . . . . . . . . . . . . . . . . . p. 81

5.6.1 Condições de Contato - Punção Hemisférico . . . . . . . . . . p. 81

5.6.2 Condições de Contato - Punção Quadrado . . . . . . . . . . . p. 85

5.6 .3 Condições de atrito . . . . . . . . . . . . . . . . . p. 89

6 Implementação Computacional p. 91

6.1 Funcionamento do Programa . . . . . . . . . . . . . . . p. 91

6.2 Estrutura do Programa . . . . . . . . . . . . . . . p. 93

6.3 Validação do Programa SHEET . . . . . . . . . . . . . . . p. 94

6.3.1 Estampagem profunda com punção hemisférico . . . . . . . p p.95

6.3.2 Estampagem profunda com punção quadrado . . . . . . . . p. 96

7 Resultados e Discussões $\quad$ p. 103

7.1 Estiramanto com punção hemisférico . . . . . . . . . . . . . p. 103

7.2 Estampagem profunda com punção quadrado . . . . . . . . . . . p. 109

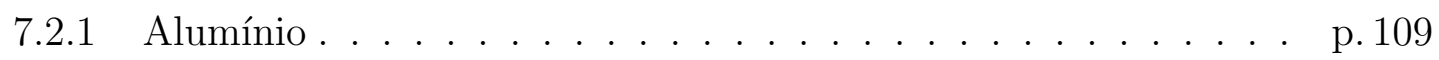

7.2 .2 Aço baixo carbono . . . . . . . . . . . . . p. 114

8 Conclusões e Sugestões para Trabalhos Futuros p. 125

8.1 Conclusões . . . . . . . . . . . . . . . . . . p. 125 
8.2 Sugestões para Trabalhos Futuros . . . . . . . . . . . . p. 126

$\begin{array}{ll}\text { Referências } & \text { p. } 127\end{array}$

Anexo A - Sub-rotinas Modificadas p. 131

A.1 Sub-rotina ASEMBL . . . . . . . . . . . . . . . p. 131

A.2 Sub-rotina STRAIN . . . . . . . . . . . . . . . . p. 143

$\begin{array}{ll}\text { Anexo B - Obtenção de } \mathbf{P}^{*} & \text { p. } 147\end{array}$ 


\section{Introdução}

\section{$1.1 \quad$ Escopo de Estudo}

A estampagem profunda, também conhecida como embutimento, é um processo de fabricação que permite a produção de peças a partir de chapas metálicas. É um processo de grande importância e de elevada produtividade, cujo campo de atuação inclui peças automobilísticas, aeronáuticas e produtos domésticos (pias, panelas, copos, etc).

A estampagem, figura 1, pode ser definida como um processo de transformação de uma chapa, no qual a chapa metálica é forçada a escoar controladamente pela atuação de um prensa-chapas para o interior de uma matriz em consequência da atuação de um punção. Tem-se como resultado uma peça oca com geometria definida pelo punção e pela matriz, e onde a espessura sofre pouca variação em relação ao seu valor inicial (Rodrigues e Martins (2005)).

Em um processo de estampagem profunda os produtos são geralmente vendidos a um valor muito baixo e em mercados altamente competitivos. Para ser competitivo o estudo do processo de estampagem deve levar em consideração que o formato do produto deve ser obtido sem defeitos, figura 2, e com uma margem de segurança suficiente para tolerar as variações nas propriedades do material conformado e nas condições de uso das ferramentas de conformação.

Para se evitarem as falhas, deve-se considerar os fatores mecânicos e metalúrgicos que controlam a estampagem profunda. Dentre os fatores mecânicos temos a forma e dimensões da peça, a máquina de conformação (tipo de prensa), a forma e dimensões 


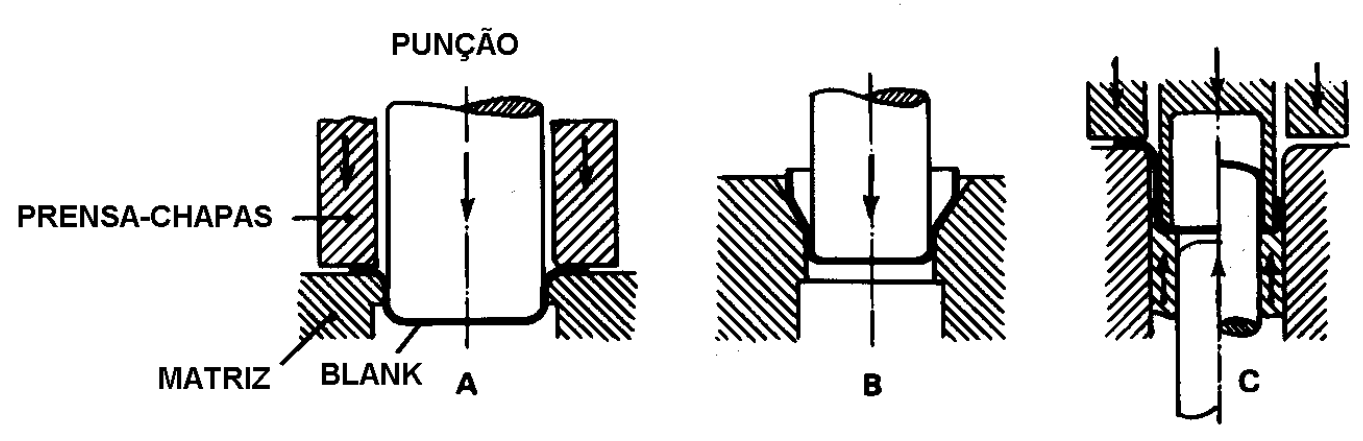

Figura 1: Estampagem profunda: (A) Primeira operação; (B)Segunda operação; (C) Extração; Em estampagem profunda, o blank é pressionado contra a matriz, através do punção, rebaixando a superfície sem alterar sensivelmente a espessura original (Kobayashi et al. (1989)).

das ferramentas. Estes fatores têm influência direta na definição dos estados de tensão e deformação existentes, em cada instante do processo, nas diversas regiões da peça em formação.

Dentre os fatores metalúrgicos, relacionados ao material do produto, tem-se a sua composição química e sua estrutura. Fatores estes que dependem dos processos de fabricação e de laminação associados aos tratamentos térmicos, os quais afetam as propriedades mecânicas da chapa e que são de importância fundamental em seu comportamento na estampagem.

O processo de fabricação de uma chapa faz com que ela tenha propriedades mecânicas dependentes de direção. No caso de chapas, propriedades distintas na direção de laminação e transversal de laminação. Este efeito é chamado de anisotropia e pode ter efeitos tanto benéficos como maléficos. Como efeito maléfico pode-se destacar a formação de orelhas e como efeito benéfico tem-se o aumento da resistência na direção normal da chapa, que reduz o afinamento da espessura.

Para analisar materiais com anisotropia deve-se adotar um critério adequado de escoamento, o qual define o limite elástico do material. Um critério de escoamento clássico que leva em conta a anisotropia do material foi proposto por Hill (1950), e é largamente 


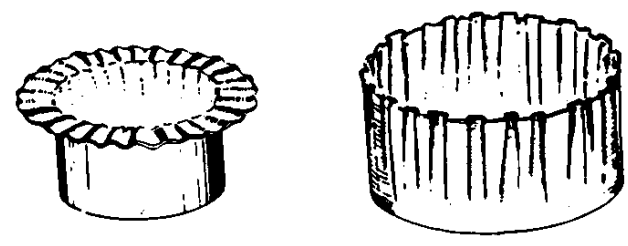

Dobras
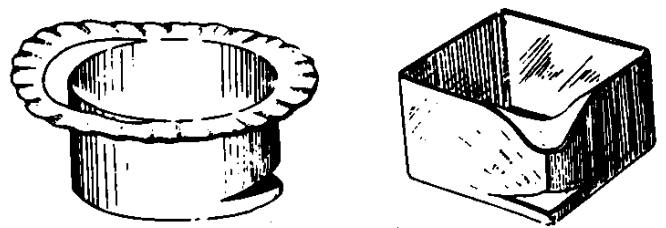

Fraturas
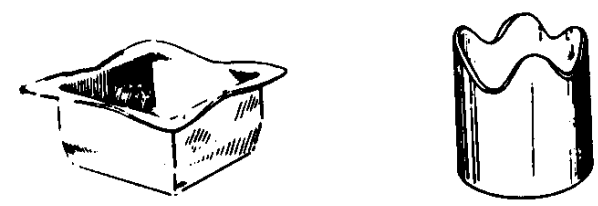

Alças

Figura 2: Defeitos observados em estampagem de peças ocas (Kobayashi et al. (1989)).

usado na análise de estampagem profunda. Tendo em vista que este critério não considera o 'comportamento anormal' observado por Woodthorpe e Pearce (1970) em chapas de alumínio, Hill (1979) propôs uma nova função para o critério de escoamento de materiais anisotrópicos. Barlat et al. (1991) propuseram um critério de seis dimensões para melhor descrever o comportamento em ligas de alumínio.

\subsection{Objetivos da Dissertação}

O presente trabalho tem como objetivo implementar os critérios de escoamentos não quadráticos para materiais anisotrópicos de Hill (1979) e de Barlat et al. (1993). Para tal, os critérios de escoamento são implementados em sua forma de deformações efetivas, em um programa acadêmico em FORTRAN denominado SHEET, desenvolvido por Toh (1983) e modificado por Olienick Filho (2003). O programa é capaz de modelar o processo de estampagem via o método dos elementos finitos utilizando o modelo constitutivo rígidoplástico e a formulação tradicional de elemento de membrana. Com os critérios não 
quadráticos faz-se um estudo da influência do critério de escoamento na distribuição de deformação na estampagem profunda e assim obtém-se um critério mais próximo do comportamento real do material.

\subsection{Conteúdo desta Dissertação}

O conteúdo desta dissertação é dividido em 8 capítulos descritos a seguir:

Capítulo 2 Apresenta um breve histórico do desenvolvimento do Método dos Elementos Finitos aplicado ao estudo da conformação mecânica. Os critérios de escoamento na análise de estampagem profunda são igualmente revistos.

Capítulo 3 Revisa conceitos da teoria da plasticidade envolvidos na descrição da deformação plástica. Apresenta-se um estudo dos critérios de escoamento implementados.

Capitulo 4 Aborda a transmissão das tensões e deformações para o caso de chapas metálicas, ilustrando-se ao final a mecânica da estampagem profunda de chapas.

Capítulo 5 Apresenta a formulação do método dos elementos finitos e sua aplicação na estampagem de chapas.

Capítulo 6 Mostra a estrutura do programa e resultados numéricos obtidos.

Capítulo 7 Mostra os resultados obtidos com a utilização do critério de escoamento de Hill (1979).

Capítulo 8 Apresenta as conclusões e sugestões para trabalhos futuros. 


\section{Revisão Bibliográfica}

A comformação de chapas metálicas é um processo altamente baseado na experiência e envolve vários ciclos de tentativa e erro. Este ciclos são mais repetidos quando há menos experiência de uso do material e da geometria da peça. Os ciclos de tentativa e erro podem ser reduzidos pela utilização de modernas técnicas de análise numérica. O uso de métodos numéricos na análise de conformação de chapas metálicas teve seu início na década de 60 .

Woo (1968) obteve soluções para a estampagem profunda com um punção hemisférico utilizando o Método das Diferenças Finitas, obtendo bons resultados comparado com os resultados experimentais. Entretanto, este método está restrito a problemas axissimétricos (Tekkaya (2000)).

Grande avanço na análise numérica veio com o uso do Método dos Elementos Finitos. Mehta e Kobayashi (1971) aplicaram o Método dos Elementos Finitos na análise elastoplástica não axissimétrica de uma chapa estirada com anisotropia normal.

Usando um modelo elasto-plástico, Wifi (1976) obteve uma solução completa para o estiramento e estampagem profunda, considerando o contato entre a chapa e o prensachapas, a matriz, e o punção.

Baseado na formulação variacional do problema de conformação de chapas e assumindo um material rígido-plástico, Kim e Kobayashi (1978) analisaram três processos axissimétricos: estiramento com pressão hidrostática, estiramento e estampagem profunda com punção hemisférico.

Wang e Budianskky (1978) desenvolveram um procedimento para o cálculo de de- 
formações para formas arbitrárias de punção e matriz, baseado na teoria não linear de elementos de casca e usando um modelo elasto-plástico.

Onate e Zienkiewics (1983) analisaram a deformação de chapas finas usando uma formulação baseada na teoria do escoamento viscoplástico aplicado ao elemento de casca fina.

Toh (1983) analisou a conformação de chapas metálicas, axissimétricas e não simétricas, usando elementos de membrana e modelo rígido-plástico, incluindo efeitos de anisotropia normal.

Germain et al. (1989) modelaram o estiramento usando um modelo rígido-viscoplástico, com anisotropia normal e elementos de membrana. Também implementaram o critério de escoamento de Hill (1979) e algoritmos especiais para tratar o contato com a matriz e a fricção de Coulomb.

Guo et al. (1990) desenvolveram um algoritmo em elementos finitos para avaliar a formabilidade em estágios iniciais de desenvolvimento do produto. O algoritmo é chamado de inverse approach, é também conhecido como one-step, pois ele usa simplificações que possibilitam o cálculo a partir do produto final para o blank inicial em apenas um passo.

Yoo et al. (1994) utilizaram elementos de membrana modificados para levar em conta o efeito da flexão, na análise de estampagem profunda, junto com um método melhorado de tratamento do contato.

Yoon et al. (1999) desenvolveram uma formulação de elementos finitos elasto-plásticos baseado na teoria de deformação incremental, para materiais com anisotropia planar. Para considerar os efeitos de flexão, e também o retorno elástico, um elemento de casca que permite grandes rotações e grandes deformações é utilizado. O critério de escoamento usado é o de Barlat et al. (1991).

Cardoso et al. (2002) desenvolveram um elemento de casca para aplicações em problemas não lineares, incluindo-se a conformação de chapas metálicas, baseada na integração 
reduzida das matrizes em nível de elemento. Este elemento possui cinco graus de liberdade e quatro nós, e incorpora materiais elasto-plásticos com anisotropia planar.

Gan e Wagoner (2004) desenvolveram um novo método para projeto de matrizes para conformação de chapas que leva em conta o efeito de retorno elástico. O método é baseado na comparação iterativa entre a forma final do produto desejado com o resultado obtido a partir de uma simulação em elementos finitos.

Manabe et al. (2008) validaram um modelo em elementos finitos que considera a rugosidade da superfície, para estudar os efeitos da rugosidade do blank e do ferramental no processo de micro estampagem profunda.

A seguir faz-se um breve histórico dos esforços para se obter um critério de escoamento que reproduza o comportamento anisotrópico de chapas

Hill (1950) propôs um critério de escoamento quadrático, generalizando o critério de escoamento de von Mises adicionando-se seis constantes de anisotropia. É um dos critérios mais usados na análise de conformação de chapas.

O critério de Hill (1950) fornece uma relação entre a tensão de escoamento sobre carregamento biaxial e a tensão de escoamento sobre carregamento uniaxial onde a tensão em carregamento biaxial deve ser menor que a tensão sobre carregamento uniaxial, para valores de $R^{1}$ menores que a unidade. Entretanto, dados experimentais de Woodthorpe e Pearce (1970) mostram que a tensão de escoamento sob carregamento biaxial de alguns alumínios é maior que a tensão de escoamento sob carregamento uniaxial, sendo conhecido como comportamento anormal do alumínio. Para levar em conta o comportamento anormal, Hill (1979) propôs um critério não-quadrático de ordem $M$, sendo uma extensão de seu critério anterior.

Outros critérios também foram propostos, como o de Hosford (1972) que trocou o expoente quadrático de Hill (1950) por uma variável, este critério pode ser considerado um caso especial de Hill (1979). Gotoh (1977) propôs uma função polinomial de quarta

\footnotetext{
${ }^{1} \mathrm{R}$ é um coeficiente de anisotropia normal
} 
ordem com nove parâmetros, que caracterizam o comportamento do material. O critério de Bassani (1977) possui dois expoentes variáveis, adicionando graus de liberdade a mais para descrever a superfície de escoamento. Considerando anisotropia planar, o critério de Barlat e Lian (1989) é uma generalização de Hosford (1972), com quatro parâmetros e um termo de cisalhamento.

Barlat et al. (1991) propuseram um critério de escoamento de seis componentes apropriado para metais poli-cristalinos com simetria ortotrópica, e que mostra ser consistente com a superfície de escoamento baseada em análise cristalográfica. Este critério foi usado por Chung e Shah (1992) para analisar o processo de estampagem de um copo cilíndrico.

Para a utilização do critério de Barlat et al. (1991) em uma formulação de elementos finitos rígido-plásticos, Barlat et al. (1993) propuseram uma taxa de deformação efetiva para metais ortotrópicos. Essa nova taxa de deformação efetiva não é conjugada (não está matematicamente relacionada) com a tesão efetiva do critério de Barlat et al. (1991), porém alguns testes numéricos mostram que são aproximadamente conjugados (Barlat e Chung (1993)).

Baseado nessa nova definição de taxa de deformação efetiva Yoon et al. (1995), usando um sistema de coordenadas naturais, simularam o processo de estampagem de um copo cilíndrico para estudar a formação de "orelhas" na liga de alumínio 2008-T4. Chung et al. (1996) usando coordenadas cartesianas, estudaram a formação de "orelhas" na liga de alumínio-lítio 2090-T3.

Barlat et al. (1991) e Barlat et al. (1993) usaram a tensão efetiva de ensaio uniaxial ou os valores de $R$ para a obtenção dos coeficientes de anisotropia. Para usar a tensão efetiva e valores de $R$ simultaneamente Barlat et al. (1997) desenvolveram um novo critério de escoamento e Chung et al. (1999) a taxa de deformação efetiva associada a esse critério de escoamento.

Barlat et al. (1997) e Chung et al. (1999) descrevem bem o comportamento plástico das chapas metálicas, porem a convexidade não foi rigorosamente provada e sua forma 
matemática não é conveniente para a implementação no método dos elementos finitos. Para contornar este fato Barlat et al. (2003) propuseram um critério para o estado plano de tensões com rigorosa prova de convexidade. Ela é baseada em duas transformações lineares da tensão desviadora e contém oito coeficientes de anisotropia.

A formulação de Barlat et al. (2003) foi extendida para o caso tridimensional por Barlat et al. (2005), contendo 18 coeficientes de anisotropia. Como resultado ela pode predizer a formação de seis a oito 'orelhas' em simulações de elementos finitos de estampagem de copos cilíndricos, como observado em Yoon et al. (2006). Mais recentemente Kim et al. (2007) propuseram uma taxa de deformação efetiva pseudo-conjugada a Barlat et al. (2003). 


\section{Elementos de Plasticidade}

\subsection{Introdução}

Os parâmetros básicos que podem ser utilizados para descrever a mecânica da deformação são a tensão, deformação e taxa de deformação. Para ilustrar estas quantidades, utiliza-se aqui um corpo de prova de formato cilíndrico com comprimento inicial $l_{0}$ e área de seção transversal $A_{0}$, figura 3 .

Submetido a uma carga axial $P$, o corpo assumirá um comprimento $l$ e uma área de seção transversal $A$. O histórico da deformação pode ser visto em um diagrama tensão x deformação, onde o comportamento do material pode ser analisado. Duas formulações são tradicionalmente utilizadas na descrição deste histórico: a Lagrangeana e a Euleriana.

$\mathrm{Na}$ formulação Lagrangeana, as coordenadas $X_{i}$ de uma partícula genérica no seu estado indeformado (variável independente) são usadas como referência. Na formulação Euleriana, as variáveis independentes são as coordenadas $x_{i}$ de um ponto material no estado deformado.

Em um estado uniaxial de tensões, tensão, deformação e taxa de deformação podem ser definidos por:

- Tensão de Cauchy (ou tensão verdadeira): $\sigma=\frac{P}{A}$

- Taxa de deformação: $\dot{\varepsilon}=\frac{i}{l}$

- Deformação (infinitesimal): $d \varepsilon=\frac{d l}{l}$ 

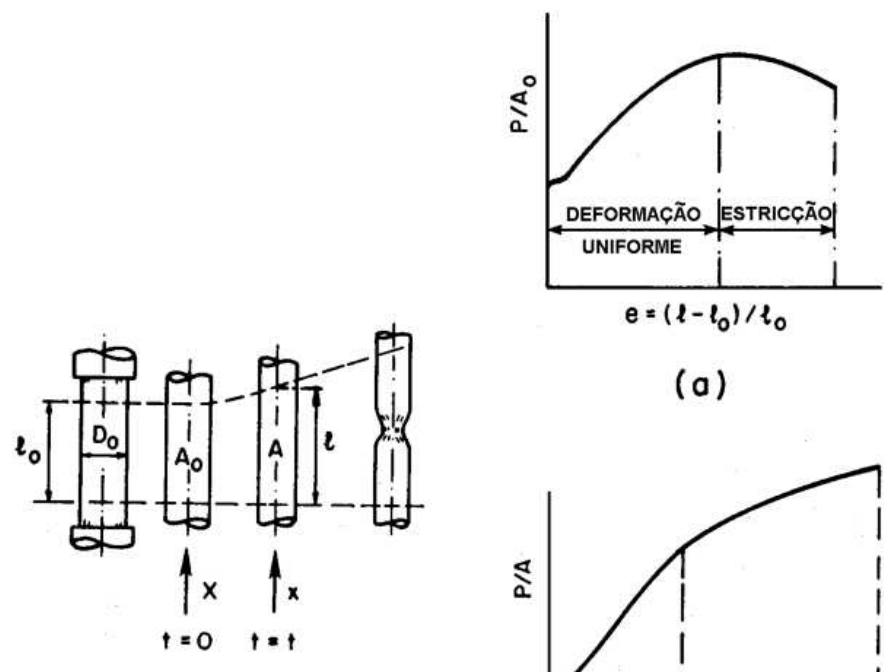

(a)

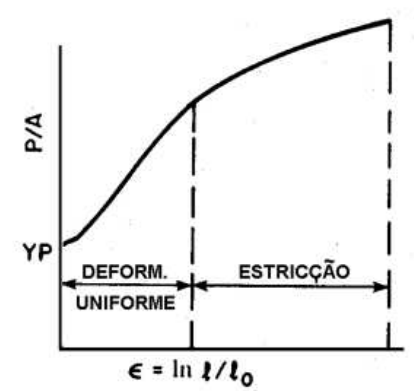

(b)

Figura 3: Diagramas de tensão: (a) Piola-Kirchhoff; (b) Cauchy (tensão verdadeira) (Kobayashi et al. (1989)).

onde o ponto denota a derivação em relação ao tempo. A deformação total pode ser obtida pela integração das deformações infinitesimais:

$$
\varepsilon=\int_{l_{0}}^{l} d \varepsilon=\ln \left(\frac{l}{l_{0}}\right)
$$

onde $\varepsilon$ é chamada de deformação natural.

A descrição Lagrangeana da deformação finita, usando tensão, deformação e taxa de deformação, pode ser expressa, tomando a posição de uma partícula na configuração deformada, em um instante $t$.

$$
x=\chi(X, t)
$$

Em um estado uniaxial de tensões, com $X$ medido longitudinalmente ao espécime,

$$
x=X+\left(\frac{l-l_{0}}{l_{0}}\right) X
$$

e o gradiente de deformação relativa, usado em engenharia é definido como:

$$
e=\frac{\partial(x-X)}{\partial X}=\frac{l-l_{0}}{l_{0}}
$$


A componente de deformação Lagrangeana $E_{11}$ é:

$$
E_{11}=\frac{1}{2}\left(\frac{\partial x}{\partial X} \frac{\partial x}{\partial X}-1\right)=\frac{1}{2}\left[(1+e)^{2}-1\right]=e+\frac{1}{2} e^{2}
$$

onde

$$
\begin{aligned}
& \dot{e}=\frac{i}{l_{0}} \\
& \dot{E_{11}}=\frac{\partial x}{\partial X} \frac{\partial \dot{x}}{\partial X}=(1+e) \dot{e} \\
& \dot{x}=\left.\frac{\partial \chi}{\partial t}\right|_{\chi=\text { const }}
\end{aligned}
$$

O tensor tensão de Piola-Kirchhoff é a tensão usada na Engenharia, definido como

$$
P_{11}=\frac{P}{A_{0}}
$$

A taxa de trabalho por unidade de volume pode ser definida nas seguintes formas:

$$
\dot{W}_{0}=\frac{P \dot{l}}{A_{0} l_{0}}=P_{11} \frac{\partial \dot{x}}{\partial X}=\frac{P_{11}}{1+e} \dot{E}_{11}
$$

e a medida de tensão correspondente à taxa de deformação Lagrangeana é:

$$
S_{11}=\frac{P_{11}}{1+e}
$$

que corresponde a um componente do tensor segundo de Piola-Kirchhoff (PK-2), o qual é simétrico.

O tensor Tensão de Piola-Kirchhoff $(P K)$ é definido considerando a área de seção transversal na configuração indeformada. Na análise dos processos de conformação metálica, formulações de fluxo e sólida envolvem a teoria das deformações finitas, daí a importância deste tensor para este estudo. Em estudos de processos que envolvem grandes deformações, a formulação sólida e de fluxo são usadas, envolvendo os tensores $P K$ e $P K$-2.

Por outro lado, em vários processos de conformação mecânica (como os que envolvem as deformações no plano, por exemplo) os estudos são baseados na deformação infinitesimal.

O tensor taxa de deformação $\dot{\varepsilon}_{i j}$ é simétrico e pode ser escrito para o caso tridimen- 
sional da seguinte forma:

$$
\dot{\varepsilon}_{i j}=\frac{1}{2}\left(\dot{u}_{i, j}+\dot{u}_{j, i}\right)
$$

onde $u_{i}$ e $u_{j}$ são os deslocamentos e o ponto denota a derivação em relação ao tempo. O tensor tensão de Cauchy pode ser representado da seguinte forma: $\sigma_{i j}$

A tensão também pode ser especificada pelos seus três componentes principais, ou, seus três invariantes tensoriais. As tensões principais $\left(\sigma_{1}, \sigma_{2}, \sigma_{3}\right)$, são as raízes da equação

$$
\sigma^{3}-I_{1} \sigma^{2}-I_{2} \sigma-I_{3}=0
$$

onde:

$$
\begin{aligned}
& I_{1}=\sigma_{x x}+\sigma_{y y}+\sigma_{z z}=\sigma_{1}+\sigma_{2}+\sigma_{3} \\
& I_{2}=-\left(\sigma_{x x} \sigma_{y y}+\sigma_{y y} \sigma_{z z}+\sigma_{z z} \sigma_{x x}\right)+\tau_{x y}^{2}+\tau_{y z}^{2}+\tau_{z x}^{2}=-\left(\sigma_{1} \sigma_{2}+\sigma_{2} \sigma_{3}+\sigma_{3} \sigma_{1}\right) \\
& I_{3}=\sigma_{x x} \sigma_{y y} \sigma_{z z}+2 \tau_{x y} \tau_{y z} \tau_{z x}-\sigma_{x x} \tau_{y z}^{2}-\sigma_{y y} \tau_{z x}^{2}-\sigma_{z z} \tau_{x y}^{2}=\sigma_{1} \sigma_{2} \sigma_{3}
\end{aligned}
$$

\subsection{Regra do Fluxo e Potencial Plástico}

A regra do fluxo fornece a relação ou a grandeza relativa dos componentes do tensor incremento das deformações plásticas ${ }^{1} \mathrm{~d} \varepsilon_{i j}^{p}$. Este incremento pode ser representado geometricamente por um vetor com nove componentes no espaço de deformações (figura 4). Pode-se também definir a direção do tensor incremento de deformação plástica $\mathrm{d} \varepsilon_{i j}^{p}$ no espaço de deformações.

Portanto, quando as deformações se estendem além do regime elástico (Lei de Hooke), as relações entre tensões e deformações são derivadas usando o conceito de potencial plástico.

\section{Regra do Fluxo e Potencial Plástico}

$$
\dot{\varepsilon}_{i j}^{p}=h \frac{\partial g}{\partial \sigma_{i j}} \dot{f} \quad \text { ou } \quad \mathrm{d} \varepsilon_{i j}^{p}=h \frac{\partial g}{\partial \sigma_{i j}} \mathrm{~d} f
$$

onde $g$ e $h$ são funções escalares dos invariantes das componentes desviatórias das tensões

\footnotetext{
${ }^{1}$ o super-escrito $p$ indica a parte plástica da deformação
} 


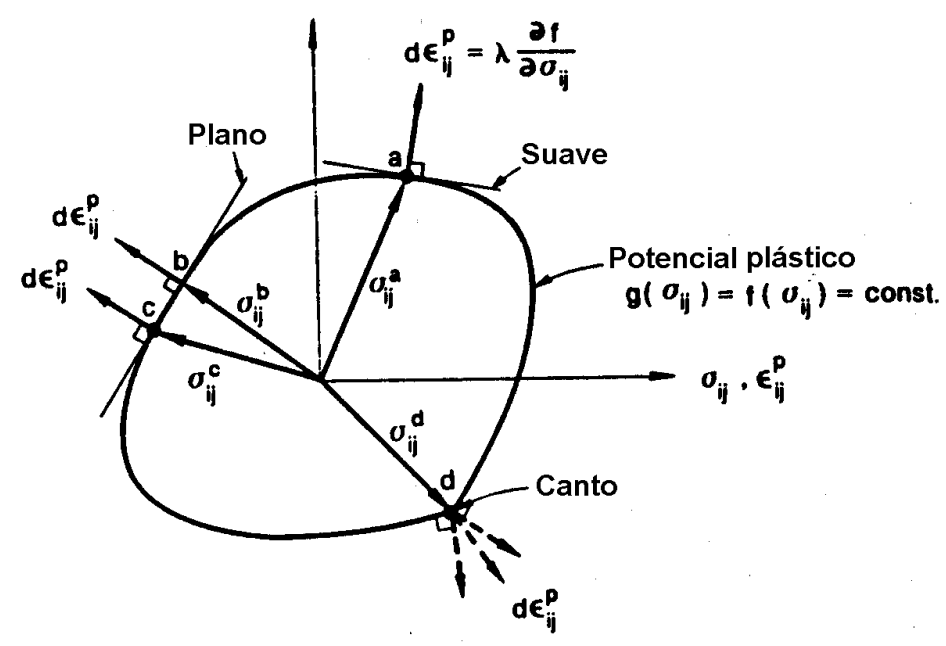

Figura 4: Ilustração Geométrica da regra associada de fluxo (Chen e Han (1988)).

e $f$ é uma função de escoamento (Kobayashi et al. (1989)).

A função $g\left(\sigma_{i j}\right)$ é uma constante que define uma superfície (hiper-superfície) do potencial plástico no espaço de tensões de nove dimensões.

Assumindo a relação $g=f$, as equações 3.12 ficam:

$$
\dot{\varepsilon}_{i j}^{p}=\frac{\partial f}{\partial \sigma_{i j}} \dot{\lambda} \quad \text { ou } \quad \mathrm{d} \varepsilon_{i j}^{p}=\frac{\partial f}{\partial \sigma_{i j}} \mathrm{~d} \lambda
$$

onde $\mathrm{d} \lambda$ um escalar positivo, chamado fator de proporcionalidade, que é diferente de zero somente quando a deformação plástica ocorre. Interpretando geometricamente $f$ como uma superfície, $\partial f / \partial \sigma_{i j}$ são os cossenos diretores de um vetor normal à tangente a esta superfície em qualquer ponto $x_{i}$. O gradiente de $f, \partial f / \partial \sigma_{i j}$ no ponto $\sigma_{i j}$ está na direção normal a esta superfície. A relação 3.13 implica que o vetor fluxo plástico d $\varepsilon_{i j}^{p}$, é posicionado normal à superfície do potencial plástico. Esta equação é denominada de regra de fluxo associada, porque o fluxo plástico é associado ao critério de escoamento, enquanto que se $f \neq g$, a equação 3.13 é chamada uma regra de fluxo não associada.

Princípio do Máximo Trabalho Plástico: o princípio do trabalho plástico fornece, como será visto, restrições adicionais à superfície de escoamento e ao vetor $\mathrm{d} \varepsilon_{i j}^{p}$. Este princípio pode ser ilustrado através das seguintes considerações: considera-se uma unidade 


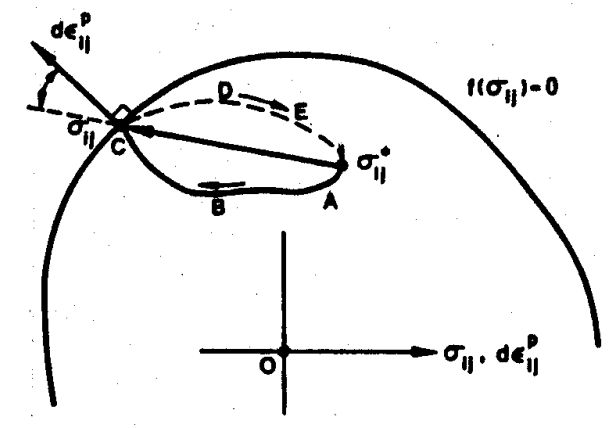

(a)

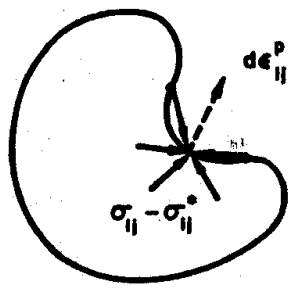

Não Permissível

(b)

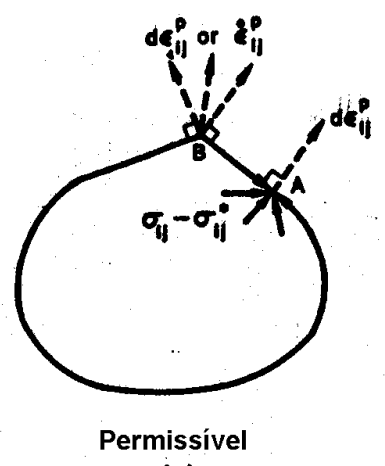

(c)

Figura 5: Convexidade da superfície de campo e normalidade do fluxo plástico. Chen e Han (1988)

de volume de material no qual exista um estado de tensões homogêneo $\sigma_{i j}^{*}$ na superfície de escoamento ou internamente à ela (figura 5 a). A seguir supõe-se que um agente externo adicione tensões ao longo do caminho $\mathrm{ABC}$, internamente à superfície até que $\sigma_{i j}$ seja alcançado na superfície de escoamento. Até este momento, somente trabalho elástico terá sido realizado . Agora, supondo que o agente externo mantenha o estado de tensões $\sigma_{i j}$ por um curto espaço de tempo. Como toda mudança puramente elástica é completamente reversível e independente do caminho, de $\sigma_{i j}^{*}$ para $\sigma_{i j}$ e de volta à $\sigma_{i j}^{*}$, toda energia elástica é recuperada. O trabalho plástico feito pelo agente externo neste ciclo de carregamentodescarregamento é um produto escalar do vetor tensão $\left(\sigma_{i j}-\sigma_{i j}^{*}\right)$ e vetor incremento de deformação plástica $\mathrm{d} \varepsilon_{i j}^{p}$.

Deste modo, o princípio do máximo trabalho plástico, é expresso , a partir da irreversibilidade e conseqüentemente, do fato de ser positivo por:

$$
\left(\sigma_{i j}-\sigma_{i j}^{*}\right) \dot{\varepsilon}_{i j}^{p} \geq 0 \quad \text { ou } \quad\left(\sigma_{i j}-\sigma_{i j}^{*}\right) \mathrm{d} \varepsilon_{i j}^{p} \geq 0
$$


A interpretação geométrica de 3.14 pode ser dada da seguinte forma: se as coordenadas das deformações plásticas são superpostas às coordenadas de tensão, como na figura 5, o produto escalar positivo requer um ângulo agudo entre o vetor tensão $\sigma_{i j}-\sigma_{i j}^{*}$ e o vetor incremento de deformação $\mathrm{d} \varepsilon_{i j}^{p}$. Uma vez que todos os possíveis vetores tensão devem satisfazer 3.14, isto conduz, inevitavelmente às seguintes conseqüências:

I - Convexidade: a superfície de escoamento deve ser convexa. Se não convexa como mostrado na figura $5 \mathrm{~b}$, as direções possíveis de $\mathrm{d} \sigma_{i j}$ cobrem mais de $180^{\circ}$ para alguns planos através de $\mathrm{d} \varepsilon_{i j}^{p}$. Então, o ângulo entre $\sigma_{i j}-\sigma_{i j}^{*}$ e $\mathrm{d} \varepsilon_{i j}^{p}$ pode ser maior que $90^{\circ}$. Como a equação 3.14 requer que o ângulo entre eles seja menor que $90^{\circ}$, estabelece-se que a superfície deve ser convexa.

II - Normalidade: o vetor incremento de deformação plástica deve ser normal à superfície de escoamento em um ponto de superfície regular e se ajustar entre normais adjacentes em um canto. Como pode ser visto na figura 5 c, se a superfície é convexa e suave em um ponto $\mathrm{A}, \mathrm{d} \varepsilon_{i j}^{p}$ deve ser normal à superfície de maneira que tenha um ângulo reto ou menor com qualquer $\sigma_{i j}-\sigma_{i j}^{*}$, e a condição 3.14 seja satisfeita. Se a superfície tem um canto no ponto B, existe uma liberdade na direção de $\mathrm{d} \varepsilon_{i j}^{p}$, mas o vetor deve se ajustar entre as normais aos pontos adjacentes ao canto de modo que 3.13 seja satisfeita.

\subsection{Critério de Escoamento}

Um critério de escoamento é definido como uma lei onde o limite de elasticidade sob qualquer combinação de tensões é expresso da seguinte forma:

$$
f\left(\sigma_{i j}\right)=C(\text { constante })
$$

Nos materiais isotrópicos, o escoamento plástico depende somente da magnitude das três tensões principais e não das suas direções. Assim, pode-se definir qualquer critério 
de escoamento na forma:

$$
f\left(I_{1}, I_{2}, I_{3}\right)=C
$$

Experimentalmente, sabe-se que o escoamento de um material, numa primeira instância, não é afetado pela pressão hidrostática $\sigma_{m}=1 / 3\left(\sigma_{1}+\sigma_{2}+\sigma_{3}\right)$. Segue então, que que o escoamento depende somente dos componentes principais $\left(\sigma_{1}^{\prime}, \sigma_{2}^{\prime}, \sigma_{3}^{\prime}\right)$ do tensor de tensões desviadoras:

$$
\sigma_{i j}^{\prime}=\sigma_{i j}-\delta_{i j} \sigma_{m}
$$

onde $\delta_{i j}(=1$ para $i=j$ e $=0$ para $i \neq j)$ é o delta de Kronecker.

Então o critério de escoamento fica na forma

$$
f\left(J_{2}, J_{3}\right)=C
$$

onde

$$
\begin{array}{r}
J_{2}=-\left(\sigma_{1}^{\prime} \sigma_{2}^{\prime}+\sigma_{2}^{\prime} \sigma_{3}^{\prime}+\sigma_{3}^{\prime} \sigma_{1}^{\prime}\right) \\
J_{3}=\sigma_{1}^{\prime} \sigma_{2}^{\prime} \sigma_{3}^{\prime}
\end{array}
$$

Dois critérios têm sido usados para a análise de deformação dos metais, considerando os isotrópicos:

\section{- Critério de Tresca:}

Critério de máxima tensão de cisalhamento

$$
\sigma_{1}-\sigma_{3}=\text { const }
$$

$\operatorname{com} \sigma_{1} \geq \sigma_{2} \geq \sigma_{3}$

\section{- Critério de von Mises}

Este critério, também conhecido como critério da energia de distorção, estabelece que o escoamento ocorre quando $J_{2}$ alcança um valor crítico, ou, que a função escoamento $f$ 


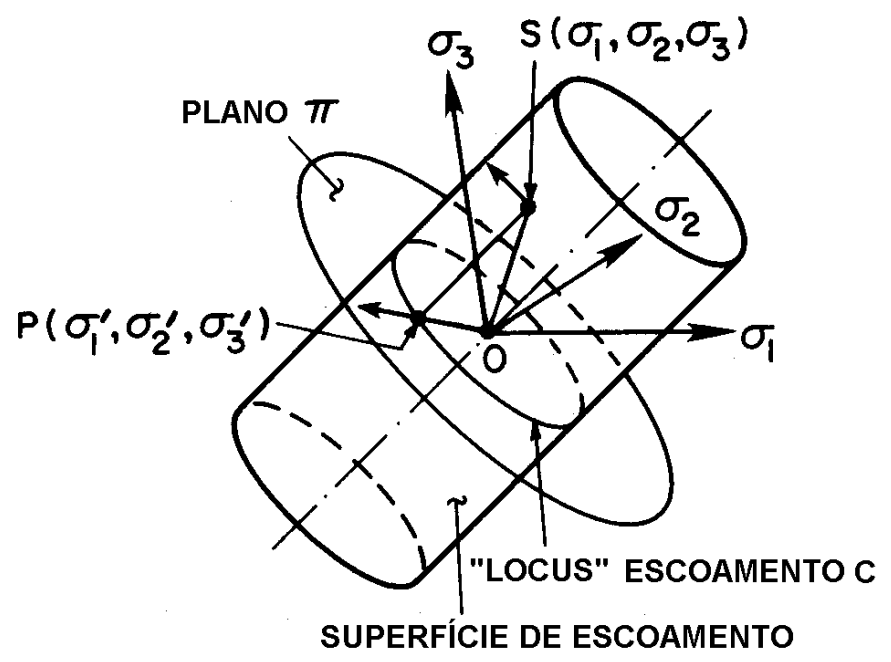

Figura 6: Representação geometrica de estado plástico de tensão no espaço (Kobayashi et al. (1989)).

da eq.(3.18) não envolve $J_{3}$ :

$$
\begin{array}{r}
J_{2}=\frac{1}{2}\left(\sigma_{1}^{\prime 2}+\sigma_{2}^{\prime 2}+\sigma_{3}^{\prime 2}\right)=\frac{1}{2} \sigma_{i j}^{\prime} \sigma_{i j}^{\prime}=k^{2} \\
\text { ou } \\
\left(\sigma_{1}-\sigma_{2}\right)^{2}+\left(\sigma_{2}-\sigma_{3}\right)^{2}+\left(\sigma_{3}-\sigma_{1}\right)^{2}=6 k^{2}
\end{array}
$$

onde $k=\frac{\sigma_{0}}{\sqrt{3}}, \sigma_{0}$ é a tensão de escoamento em um ensaio de tração simples.

Deve-se notar que o critério de escoamento dado pela equação acima depende, no processo prévio, da deformação plástica. Se é assumido que ocorra encruamento, e só trabalho plástico seja feito, então a pressuposição de que o critério de escoamento é independente da componente hidrostática, implica em que não haja mudança de volume na deformação plástica.

A representação geométrica do estado de tensão acima pode ser feia através de um vetor em um espaço tridimensional de tensões, onde as tensões principais são tomadas segundo os eixos ortogonais do sistema cartesiano (figuras 6 e 7).

Neste estudo, estes critérios não são adequados. A matéria prima empregada em 


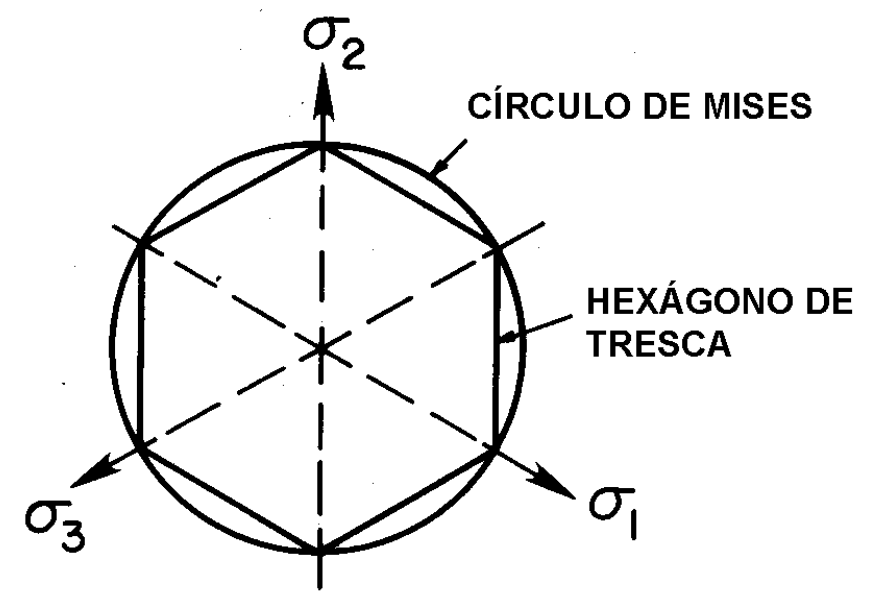

Figura 7: "Locci" do escoamento no plano p para o critério de distorção de energia e tensão cisalhante (Kobayashi et al. (1989)).

processos de estampagem, as chapas metálicas, são produzidas por meio de laminação. Assim, o comportamento observado neste material é anisotrópico. Hill (1950) estabeleceu um critério adequado para estes casos.

\subsubsection{Equações de Prandtl-Reuss e Levy-Mises}

Para materiais elasto-plásticos, as equações constitutivas relacionam a taxa de deformação à taxa de tensão, em vez de valor das tensões. Pela aplicação da equação (3.21) na equação de $\dot{\varepsilon}_{i j}$ e considerando a função de escoamento de von Mises $f\left(\sigma_{i j}\right)=J_{2}-k^{2}=0$ como potencial plástico, então a regra de fluxo tem a forma (Toh (1983)):

$$
\dot{\varepsilon}_{i j}^{p}=\sigma_{i j}^{\prime} \dot{\lambda} \quad \text { ou } \quad d \varepsilon_{i j}^{p}=\sigma_{i j}^{\prime} d \lambda
$$

e considerando ainda:

$$
\frac{\partial f}{\partial \sigma_{i j}}=\frac{\partial f}{\partial \sigma_{k l}^{\prime}} \frac{\partial \sigma_{k l}^{\prime}}{\partial \sigma_{i j}}=\sigma_{i j}^{\prime}
$$

onde os subscritos repetidos $k$ e $l$ indicam somatório com relação a estas quantidades. As equações $\dot{\varepsilon}_{i j}^{p}=\sigma_{i j}^{\prime} \dot{\lambda}$ ou $d \varepsilon_{i j}^{p}=\sigma_{i j}^{\prime} d \lambda$ podem ser escritas na forma:

$$
\frac{\dot{\varepsilon}_{x}^{p}}{\sigma_{x}^{\prime}}=\frac{\dot{\varepsilon}_{y}^{p}}{\sigma_{y}^{\prime}}=\frac{\dot{\varepsilon}_{z}^{p}}{\sigma_{z}^{\prime}}=\frac{\dot{\gamma}_{x y}^{p}}{2 \tau_{x y}}=\frac{\dot{\gamma}_{y z}^{p}}{2 \tau_{y z}}=\frac{\dot{\gamma}_{z x}^{p}}{2 \tau_{z x}}=\dot{\lambda} \quad \text { ou } \frac{d \varepsilon_{x}}{\sigma_{x}^{\prime}}=\frac{d \varepsilon_{y}}{\sigma_{y}^{\prime}}=d \lambda
$$


em que $d \lambda$ é um fator de proporcionalidade com o valor:

$$
d \lambda\left\{\begin{array}{l}
=0 \text { para } J_{2}<k^{2} \text { ou } J_{2} \text {, mas } d J_{2}<0 \\
>0 \text { para } J_{2}=k^{2} \text { e } \quad d J_{2}=0
\end{array}\right.
$$

Combinando componentes elásticas da taxa de deformação e componentes plásticas, na forma $\dot{\varepsilon}_{i j}=\dot{\varepsilon}_{i j}^{p}+\dot{\varepsilon}_{i j}^{e}$, obtemos as equações de Prandtl-Reuss, para sólidos elastoplásticos:

$$
\dot{\varepsilon}_{i j}=\sigma_{i j}^{\prime} \dot{\lambda}+\frac{1}{2 G} \dot{\sigma}_{i j}{ }^{\prime}+\delta_{i j}\left(\frac{1-2 \nu}{E}\right) \dot{\sigma}_{m m} \quad \text { ou } \quad d \varepsilon_{i j}=\sigma_{i j}^{\prime} d \lambda+\frac{1}{2 G}{\dot{\sigma_{i j}}}^{\prime}+\delta_{i j}\left(\frac{1-2 \nu}{E}\right) \dot{\sigma}_{m m}
$$

Para materiais rígido-plástico, assume-se que $\dot{\varepsilon}_{i j} \cong \varepsilon_{i j}^{p}$ e obtém-se as equações de Levy-Mises, com a remoção do superescrito $p$ de (3.22). Estas equações são expressas em termo de componentes $\sigma_{i j}$, por três equações do tipo:

$$
\dot{\varepsilon}_{x}=\left\{\sigma_{x}-\frac{1}{3}\left(\sigma_{x}+\sigma_{y}+\sigma_{z}\right)\right\} \dot{\lambda}=\left\{\sigma_{x}-\frac{1}{2}\left(\sigma_{y}+\sigma_{z}\right)\right\} \frac{2}{3} \dot{\lambda}
$$

e três do tipo:

$$
\dot{\varepsilon}_{x y}=\frac{\dot{\gamma}_{x y}}{2}=\tau_{x y} \dot{\lambda}
$$

\subsection{Critério de Hill 50}

Um material ortotrópico tem três planos mutuamentes ortogonais de simetria em cada ponto. A interseção destes três planos são conhecidas como os eixos pricipais de anisotropia. O critério de escoamento proposto por Hill (1950), referenciado em relação a estes três eixos é dado na forma:

$$
2 f\left(\sigma_{i j}\right)=F\left(\sigma_{y}-\sigma_{z}\right)^{2}+G\left(\sigma_{z}-\sigma_{x}\right)^{2}+H\left(\sigma_{x}-\sigma_{y}\right)^{2}+2 L \tau_{y z}^{2}+2 M \tau_{z x}^{2}+2 N \tau_{x y}^{2}=1
$$

onde F, G, H, L, M, N, são os parâmetros característicos do estado atual de anisotropia. A omissão de termos lineares e a aparição somente de diferenças entre componentes normais de tensão, implica assumir que as respostas do material são iguais em tração e compressão 
e que tensões hidrostáticas não infuenciam no escoamento.

Os parâmetros de material podem ser determinados a partir de três simples ensaios de tração nas direções dos eixos principais de anisotropia e três ensaios simples de cisalhamento entre estes eixos de simetria.

A partir da equação 3.28, Hill propôs a definição de tensão equivalente como

$$
\begin{gathered}
2 f\left(\sigma_{i j}\right)=2 \bar{\sigma}^{2} \quad, \text { ou } \\
\bar{\sigma}=\sqrt{\frac{3}{2}}\left(\frac{F\left(\sigma_{y}-\sigma_{z}\right)^{2}+G\left(\sigma_{z}-\sigma_{x}\right)^{2}+H\left(\sigma_{x}-\sigma_{y}\right)^{2}+2 L \tau_{y z}^{2}+2 M \tau_{z x}^{2}+2 N \tau_{x y}^{2}}{F+G+H}\right)^{1 / 2}
\end{gathered}
$$

Se a função de escoamento, $f\left(\sigma_{i j}\right)$, é o potencial plástico, os incrementos de deformação podem ser derivados pela função

$$
\mathrm{d} \varepsilon_{i j}=\frac{\partial f\left(\sigma_{i j}\right)}{\partial \sigma_{i j}} \mathrm{~d} \lambda^{\prime}
$$

onde $\mathrm{d} \lambda^{\prime}$ é uma constante não negativa a condição de incompressibilidade, $\mathrm{d} \varepsilon_{x}+\mathrm{d} \varepsilon_{y}+\mathrm{d} \varepsilon_{z}=$ 0 é satisfeita.

Hill assumiu, para materiais rígido-plásticos, que $\bar{\sigma}$ é uma função do trabalho plástico, sendo o trabalho plástico por unidade de volume

$$
\mathrm{d} w=\sigma_{i j} \mathrm{~d} \varepsilon_{i j}=\bar{\sigma} \mathrm{d} \bar{\varepsilon}
$$

e dessa relação temos uma expressão generalizada para o incremento de deformação efetiva, $\mathrm{d} \bar{\varepsilon}$

$$
\mathrm{d} \bar{\varepsilon}=\sqrt{\frac{3}{2}}(F+G+H)^{1 / 2}\left(F\left(F \frac{G \mathrm{~d} \bar{\varepsilon}_{y}-H \bar{\varepsilon}_{z}}{F G+G H+H F}\right)^{2}+\cdots+\frac{2 \mathrm{~d} \bar{\varepsilon}_{y z}^{2}}{L}+\cdots\right)^{1 / 2}
$$

Na condição de estado plano de tensão a equação (3.28) reduz-se a

$$
2 f\left(\sigma_{i j}\right)=2 \bar{\sigma}^{2}=(G+H) \sigma_{x}^{2}-2 H \sigma_{x} \sigma_{y}+(H+F) \sigma_{y}^{2}+2 N \sigma_{x y}^{2}
$$


com parâmetros de anisotropia sendo

$$
R_{0}=\frac{H}{G}, R_{90}=\frac{H}{F}, R_{45}=\frac{2 N-(F+G)}{2(F+G)}
$$

onde $R_{0}, R_{45}$ e $R_{90}$ são os valores $\mathrm{R}$ na direção paralela, a $45^{\circ}$ e perpendicular a direção de laminação na chapa, respectivamente. O valor R é definido como sendo a razão entre a deformação verdadeira na largura e a deformação verdadeira na espessura na região de alongamento uniforme em um corpo de prova submetido a um ensaio de tração, isto é

$$
R=\frac{\ln \frac{w_{0}}{w}}{\ln \frac{t_{0}}{t}}=\frac{\ln \frac{w_{0}}{w}}{\ln \frac{w l}{w_{0} l_{0}}}
$$

onde $w, l$ e $t$ são respectivamente as largura, comprimentos e espessura do corpo de prova.

Na conformação de chapas metálicas, a abordagem ideal é considerar a condição isotrópica para o plano da chapa e uma anisotropia rotacional simétrica em torno do eixo $z$ (espessura). Tem-se a chamada isotropia planar.

$$
N=F+2 H=G+2 H,
$$

e

$$
G=F
$$

Então, aplicando a equação (3.30), no estado plano de tensão, temos

$$
\begin{aligned}
& \mathrm{d} \varepsilon_{x}=\left((H+G) \sigma_{x}-H \sigma_{y}\right) \mathrm{d} \lambda^{\prime} \\
& \mathrm{d} \varepsilon_{y}=\left((H+G) \sigma_{y}-H \sigma_{x}\right) \mathrm{d} \lambda^{\prime} \\
& \mathrm{d} \varepsilon_{x y}=(G+2 H) \sigma_{x y} \mathrm{~d} \lambda^{\prime}
\end{aligned}
$$

Pela definição de $R$, junto com as equações (3.37), a equação (3.38) pode ser expressa em termos de $R$ como 


$$
\begin{aligned}
& \mathrm{d} \varepsilon_{x}=\left((1+R) \sigma_{x}-R \sigma_{y}\right) \mathrm{d} \lambda \\
& \mathrm{d} \varepsilon_{y}=\left((1+R) \sigma_{y}-R \sigma_{x}\right) \mathrm{d} \lambda \\
& \mathrm{d} \varepsilon_{x y}=(1+2 R) \sigma_{x y} \mathrm{~d} \lambda \\
& \mathrm{d} \varepsilon_{z}=-\left(\mathrm{d} \varepsilon_{x}+\mathrm{d} \varepsilon_{y}\right)
\end{aligned}
$$

onde

$$
\mathrm{d} \lambda=F \mathrm{~d} \lambda^{\prime}=G \mathrm{~d} \lambda^{\prime}
$$

$\mathrm{Ou}$

$$
\frac{\mathrm{d} \varepsilon_{x}}{(1+R) \sigma_{x}-R \sigma_{y}}=\frac{\mathrm{d} \varepsilon_{y}}{(1+R) \sigma_{y}-R \sigma_{x}}=\frac{\mathrm{d} \varepsilon_{x y}}{(1+2 R) \sigma_{x y}}=\mathrm{d} \lambda
$$

A tensão equivalente, equação (3.29), em termos de R fica

$$
\bar{\sigma}=\sqrt{\frac{3(1+R)}{2(2+R)}} \sqrt{\sigma_{x}^{2}+\sigma_{y}^{2}-\frac{2 R}{1+R} \sigma_{x} \sigma_{y}+\frac{2(1+2 R)}{1+R} \sigma_{x y}^{2}}
$$

A equação 3.42 vem do fato de $F+G+H=3$ e que a isotropia planar $F=G$ e $N=F+2 H$ e considerando-se $R_{0}=R_{90}=R_{45}=R, H / F=H / G=R$ e $N / G=1+2 R$.

Do trabalho plástico, equação (3.31), com (3.39) e (3.42), a constante não negativa $\mathrm{d} \lambda$ é determinada como

$$
\mathrm{d} \lambda=\frac{1}{1+R} \frac{\mathrm{d} \bar{\varepsilon}}{\bar{\sigma}}
$$

e o incremento de deformação efetiva

$$
\mathrm{d} \bar{\varepsilon}=\sqrt{\frac{2}{3} \frac{(1+R)(2+R)}{(1+2 R)}} \sqrt{\mathrm{d} \varepsilon_{x}^{2}+\mathrm{d} \varepsilon_{y}^{2}-\frac{2 R}{1+R} \mathrm{~d} \varepsilon_{x} \mathrm{~d} \varepsilon_{y}+\frac{2}{1+R} \mathrm{~d} \varepsilon_{x y}^{2}}
$$

A tensão equivalente pode ser redefinida como

$$
\begin{aligned}
& \bar{\sigma}=\sqrt{\sigma_{x}^{2}+\sigma_{y}^{2}-\frac{2 R}{1+R} \sigma_{x} \sigma_{y}+\frac{2(1+2 R)}{1+R} \sigma_{x y}^{2}} \\
& =\sqrt{\frac{3}{2} \boldsymbol{\sigma}^{T} \mathbf{D}_{1} \boldsymbol{\sigma}}
\end{aligned}
$$


onde, por conveniência, utilizou-se a notação matricial, com

$$
\boldsymbol{\sigma}^{T}=\left[\begin{array}{lll}
\sigma_{x} & \sigma_{y} & \sigma_{z}
\end{array}\right]
$$

e

$$
\mathbf{D}_{1}=\frac{2}{3(1+R)}\left(\begin{array}{ccc}
1+R & -R & 0 \\
-R & 1+R & 0 \\
0 & 0 & 2(1+2 R)
\end{array}\right)
$$

com o incremento de deformação efetiva correspondente

$$
\begin{aligned}
& \mathrm{d} \bar{\varepsilon}=\frac{(1+R)}{\sqrt{(1+2 R)}} \sqrt{\mathrm{d} \varepsilon_{x}^{2}+\mathrm{d} \varepsilon_{y}^{2}-\frac{2 R}{1+R} \mathrm{~d} \varepsilon_{x} \mathrm{~d} \varepsilon_{y}+\frac{2}{1+R} \mathrm{~d} \varepsilon_{x y}^{2}} \\
& =\sqrt{\frac{2}{3} \mathrm{~d} \boldsymbol{\varepsilon}^{T} \mathbf{D} \mathrm{d} \boldsymbol{\varepsilon}}
\end{aligned}
$$

onde

$$
\mathrm{d} \varepsilon^{T}=\left[\begin{array}{lll}
\mathrm{d} \varepsilon_{x} & \mathrm{~d} \varepsilon_{y} & \mathrm{~d} \varepsilon_{x y}
\end{array}\right]
$$

e

$$
\mathbf{D}=\frac{3}{2} \frac{1+R}{1+2 R}\left(\begin{array}{ccc}
1+R & R & 0 \\
R & 1+R & 0 \\
0 & 0 & 2
\end{array}\right)
$$

O valor de $R$ usado é uma média dos valores de $R_{0}, R_{45}$ e $R_{90}$, ou seja

$$
R=\frac{1}{4}\left(R_{0}+2 R_{45}+R_{90}\right)
$$

\subsection{Critério de Hill 79}

Para a isotropia no plano $(F=G)$, a equação (3.33) reduz-se, em termos das tensões principais, a

$$
F\left(\sigma_{1}^{2}+\sigma_{2}^{2}\right)+H\left(\sigma_{1}-\sigma_{2}\right)^{2}=2 \bar{\sigma}^{2}
$$


E aplicando a equação (3.30) temos

$$
\mathrm{d} \varepsilon_{1}=\frac{\bar{\varepsilon}}{2 \bar{\sigma}}\left[(F+H) \sigma_{1}-H \sigma_{2}\right] \quad \mathrm{d} \varepsilon_{2}=\frac{\bar{\varepsilon}}{2 \bar{\sigma}}\left[(F+H) \sigma_{2}-H \sigma_{1}\right]
$$

Em tração uniaxial $\left(\sigma_{1}, \sigma_{2}\right)=\left(\sigma_{u}, 0\right)$, então $(3.52)$ e (3.53) fornecem,

$$
(F+H) \sigma_{u}^{2}=2 \bar{\sigma}^{2} \quad \text { e } \quad \frac{\mathrm{d} \varepsilon_{1}}{\mathrm{~d} \varepsilon_{2}}=-\frac{F+H}{H}
$$

Em tração biaxial idêntica $\left(\sigma_{1}, \sigma_{2}\right)=\left(\sigma_{b}, \sigma_{b}\right)$, temos $2 F \sigma_{b}^{2}=2 \bar{\sigma}^{2}$, e

$$
\left(\frac{\sigma_{b}}{\sigma_{u}}\right)^{2}=\frac{(F+H)}{2 F}=\frac{1}{2}\left(1+\frac{H}{F}\right)=\frac{1}{2}(1+R)
$$

Woodthorpe e Pearce (1970) encontraram entretanto $R<1$ e $\sigma_{b} / \sigma_{u}>1$ para o alumínio. Estas inequalidades estão em desacordo com a equação (3.55), então Hill (1979) propôs o seguinte critério de escoamento não quadrático:

$F\left|\sigma_{2}-\sigma_{3}\right|^{M}+G\left|\sigma_{3}-\sigma_{1}\right|^{M}+H\left|\sigma_{1}-\sigma_{2}\right|^{M}+A\left|2 \sigma_{1}-\sigma_{2}-\sigma_{3}\right|^{M}+B\left|2 \sigma_{2}-\sigma_{1}-\sigma_{3}\right|^{M}+C\left|2 \sigma_{3}-\sigma_{1}-\sigma_{2}\right|^{M}=\bar{\sigma}^{M}$

com $M>1$ e seis coeficientes que caracterizam a anisotropia. Quando $M=2$ temos o critério de Hill (1950).

Hill (1979) propôs 4 casos especiais da equação (3.56) para isotropia planar e valores particulares de coeficientes

Caso 1: $A=B=H=0$ e $F=G$

$$
C\left|\sigma_{1}+\sigma_{2}\right|^{M}+F\left(\left|\sigma_{1}\right|^{M}+\left|\sigma_{2}\right|^{M}\right)=\bar{\sigma}^{M}
$$

Caso 2: $C=F=G=0$ e $A=B$

$$
A\left(\left|2 \sigma_{1}-\sigma_{2}\right|^{M}+\left|2 \sigma_{2}-\sigma_{1}\right|^{M}\right)+H\left|\sigma_{1}-\sigma_{2}\right|^{M}=\bar{\sigma}^{M}
$$


Caso 3: $C=H=0, F=G$ e $A=B$

$$
A\left(\left|2 \sigma_{1}-\sigma_{2}\right|^{M}+\left|2 \sigma_{2}-\sigma_{1}\right|^{M}\right)+F\left(\left|\sigma_{1}\right|^{M}+\left|\sigma_{2}\right|^{M}\right)=\bar{\sigma}^{M}
$$

Caso 4: $A=B=F=G=0$

$$
C\left|\sigma_{1}+\sigma_{2}\right|^{M}+H\left|\sigma_{1}-\sigma_{2}\right|^{M}=\bar{\sigma}^{M}
$$

Para o caso 4, (3.60), no lugar da equação (3.54),

$$
(C+H) \sigma_{u}^{M}=\bar{\sigma}^{M} \quad \text { e } \quad \frac{H}{C}=1+2 R
$$

e o caso 4 é reescrito

$$
\left|\sigma_{1}+\sigma_{2}\right|^{M}+(1+2 R)\left|\sigma_{1}-\sigma_{2}\right|^{M}=2(1+R) \bar{\sigma}_{u}^{M}
$$

e no lugar de 3.55

$$
\left(\frac{\sigma_{b}}{\sigma_{u}}\right)^{M}=\frac{1+R}{2^{M-1}}
$$

O comportamento previsto é portanto anômalo com $M$, tal que $\sigma_{b} / \sigma_{u}<1$ quando $R>1$ ou $\sigma_{b} / \sigma_{u}>1$ quando $R<1$.

O incremento de deformação efetivo associado ao caso 4 pode ser obtido da regra do fluxo (expressa aqui em função das deformações principais)

$$
\begin{array}{r}
\frac{\mathrm{d} \varepsilon_{1}+\mathrm{d} \varepsilon_{2}}{\left|\sigma_{1}+\sigma_{2}\right|^{M-1}}=\frac{\mathrm{d} \varepsilon_{1}-\mathrm{d} \varepsilon_{2}}{(1+2 R)\left|\sigma_{1}-\sigma_{2}\right|^{M-1}}=\frac{\mathrm{d} \bar{\varepsilon}}{(1+R) \sigma_{u}^{M-1}}=\mathrm{d} \lambda \\
\sigma_{u} \mathrm{~d} \bar{\varepsilon}=\sigma_{1} \mathrm{~d} \varepsilon_{1}+\sigma_{2} \mathrm{~d} \varepsilon_{2}=\frac{1}{2}\left(\sigma_{1}+\sigma_{2}\right)\left(\mathrm{d} \varepsilon_{1}+\mathrm{d} \varepsilon_{2}\right)+\frac{1}{2}\left(\sigma_{1}-\sigma_{2}\right)\left(\mathrm{d} \varepsilon_{1}-\mathrm{d} \varepsilon_{2}\right)
\end{array}
$$

Então d $\bar{\varepsilon}$ é obtido das equações (3.62) e (3.64) eliminando-se $\sigma_{1}$ e $\sigma_{2}$

$$
\mathrm{d} \bar{\varepsilon}=\frac{1}{2}(2(1+R))^{1 / M}\left(\left|\mathrm{~d} \varepsilon_{1}+\mathrm{d} \varepsilon_{2}\right|^{M /(M-1)}+(1+2 R)^{1 /(M-1)}\left|\mathrm{d} \varepsilon_{1}-\mathrm{d} \varepsilon_{2}\right|^{M /(M-1)}\right)^{(M-1) / M}
$$


Lian et al. (1989) obtiveram os incrementos de deformação efetivos para os demais casos.

Sendo as deformações principais dadas por

$$
\begin{aligned}
& \mathrm{d} \varepsilon_{1}=\frac{1}{2}\left(\mathrm{~d} \varepsilon_{x}+\mathrm{d} \varepsilon_{y}+\sqrt{\mathrm{d} \varepsilon_{x}^{2}+\mathrm{d} \varepsilon_{x y}^{2}-2 \mathrm{~d} \varepsilon_{x} \mathrm{~d} \varepsilon_{y}+\mathrm{d} \varepsilon_{y}^{2}}\right) \\
& \mathrm{d} \varepsilon_{2}=\frac{1}{2}\left(\mathrm{~d} \varepsilon_{x}+\mathrm{d} \varepsilon_{y}-\sqrt{\mathrm{d} \varepsilon_{x}^{2}+\mathrm{d} \varepsilon_{x y}^{2}-2 \mathrm{~d} \varepsilon_{x} \mathrm{~d} \varepsilon_{y}+\mathrm{d} \varepsilon_{y}^{2}}\right)
\end{aligned}
$$

e o módulo dado por

$$
\begin{aligned}
& \left.\left|\mathrm{d} \varepsilon_{1}+\mathrm{d} \varepsilon_{2}\right|=\sqrt{(}\left(\mathrm{d} \varepsilon_{1}+\mathrm{d} \varepsilon_{2}\right)^{2}\right) \\
& \left.\left|\mathrm{d} \varepsilon_{1}-\mathrm{d} \varepsilon_{2}\right|=\sqrt{(}\left(\mathrm{d} \varepsilon_{1}-\mathrm{d} \varepsilon_{2}\right)^{2}\right)
\end{aligned}
$$

O incremento de deformação efetivo fica

$$
\begin{aligned}
& \mathrm{d} \bar{\varepsilon}=\frac{1}{2}(2(1+R))^{1 / M}\left(\left(\left(\mathrm{~d} \varepsilon_{x}+\mathrm{d} \varepsilon_{y}\right)^{2}\right)^{M / 2(M-1)}+\right. \\
& \left.+(1+2 R)^{1 /(M-1)}\left(4 \mathrm{~d} \varepsilon_{x y}^{2}+\left(\mathrm{d} \varepsilon_{x}-\mathrm{d} \varepsilon_{y}\right)^{2}\right)^{M / 2(M-1)}\right)^{(M-1) / M}
\end{aligned}
$$

Em forma matricial

$\mathrm{d} \bar{\varepsilon}=\frac{1}{2}(2(1+R))^{1 / M}\left(\left(\mathrm{~d} \boldsymbol{\varepsilon}^{T} \mathbf{D}_{\mathbf{H} \mathbf{1}} \mathrm{d} \boldsymbol{\varepsilon}\right)^{M / 2(M-1)}+(1+2 R)^{1 /(M-1)}\left(\mathrm{d} \boldsymbol{\varepsilon}^{T} \mathbf{D}_{\mathbf{H} \mathbf{2}} \mathrm{d} \boldsymbol{\varepsilon}\right)^{M / 2(M-1)}\right)^{(M-1) / M}$

com

$$
\mathbf{D}_{\mathbf{H} 2}=\left(\begin{array}{ccc}
1 & -1 & 0 \\
-1 & 1 & 0 \\
0 & 0 & 4
\end{array}\right)
$$

e

$$
\mathbf{D}_{\mathbf{H} 1}=\left(\begin{array}{lll}
1 & 1 & 0 \\
1 & 1 & 0 \\
0 & 0 & 0
\end{array}\right)
$$

\subsection{Critério de Barlat}

O critério de Barlat et al. (1991) para o caso tridimensional é definido como

$$
\Psi=\left|S_{1}-S_{2}\right|^{\varrho}+\left|S_{2}-S_{3}\right|^{\varrho}+\left|S_{3}-S_{1}\right|^{\varrho}=2 \bar{\sigma}^{\varrho}
$$


onde $S_{i=1,2,3}$ são os autovalores da matriz simetrica $\mathbf{S}$ definida em relação aos componentes de tensão de Cauchy

$$
\mathbf{S}=\left(\begin{array}{ccc}
\frac{c_{3}\left(\sigma_{x x}-\sigma_{y y}\right)-c_{2}\left(\sigma_{z z}-\sigma_{x x}\right)}{3} & c_{6} \sigma_{x y} & c_{5} \sigma_{z x} \\
c_{6} \sigma_{x y} & \frac{c_{1}\left(\sigma_{y y}-\sigma_{z z}\right)-c_{3}\left(\sigma_{x x}-\sigma_{y y}\right)}{3} & c_{4} \sigma_{z y} \\
c_{5} \sigma_{z x} & c_{4} \sigma_{z y} & \frac{c_{2}\left(\sigma_{z z}-\sigma_{x x}\right)-c_{1}\left(\sigma_{y y}-\sigma_{z z}\right)}{3}
\end{array}\right)
$$

O coeficientes $c_{i=1 \sim 6}$ representam a propriedades anisotrópicas. O expoente ' $\varrho$ ' é associado à estrutura cristalina.

A deformação incremental efetiva, d $\bar{\varepsilon}$, associada a tensão efetiva, pode ser obtida analiticamente através do princípio de trabalho equivalente

$$
\bar{\sigma} \mathrm{d} \bar{\varepsilon}=\sigma_{i j} \mathrm{~d} \varepsilon_{i j}
$$

porém este problema não possui uma solução analítica. Então, Barlat et al. (1993) propuseram uma deformação incremental efetiva que não é conjugada com a tensão efetiva, porém alguns resultados numéricos mostram que são aproximadamente conjugados (Barlat e Chung (1993)).

O critério de Barlat et al. (1993) para o caso tridimensional é definido como

$$
2(k \mathrm{~d} \bar{\varepsilon})^{\zeta}=|p|^{\zeta}+|q|^{\zeta}+|p+q|^{\zeta}
$$

onde

$$
p=\frac{2 \mathrm{~d} \varepsilon_{1}-\mathrm{d} \varepsilon_{2}-\mathrm{d} \varepsilon_{3}}{3} \quad \text { e } \quad q=\frac{2 \mathrm{~d} \varepsilon_{2}-\mathrm{d} \varepsilon_{3}-\mathrm{d} \varepsilon_{1}}{3}
$$

sendo $k$ um número real usado como fator de correção. d $\varepsilon_{i=1,2,3}$ são os autovalores da matriz simetrica $\mathbf{L}$, definida pelas componentes do tensor de deformações incrementais $\mathrm{d} \varepsilon_{i j}^{p}$

$$
\mathbf{L}=\left(\begin{array}{ccc}
\frac{c_{3}\left(\mathrm{~d} \varepsilon_{x x}^{p}-\mathrm{d} \varepsilon_{y y}^{p}\right)-c_{2}\left(\mathrm{~d} \varepsilon_{z z}^{p}-\mathrm{d} \varepsilon_{x x}^{p}\right)}{3} & c_{6} \mathrm{~d} \varepsilon_{x y}^{p} & c_{5} \mathrm{~d} \varepsilon_{z x}^{p} \\
c_{6} \mathrm{~d} \varepsilon_{x y}^{p} & \frac{c_{1}\left(\mathrm{~d} \varepsilon_{y y}^{p}-\mathrm{d} \varepsilon_{z z}^{p}\right)-c_{3}\left(\mathrm{~d} \varepsilon_{x x}^{p}-\mathrm{d} \varepsilon_{y y}^{p}\right)}{3} & c_{4} \mathrm{~d} \varepsilon_{z y}^{p} \\
c_{5} \mathrm{~d} \varepsilon_{z x}^{p} & c_{4} \mathrm{~d} \varepsilon_{z y}^{p} & \frac{c_{2}\left(\mathrm{~d} \varepsilon_{z z}^{p}-\mathrm{d} \varepsilon_{x x}^{p}\right)-c_{1}\left(\mathrm{~d} \varepsilon_{y y}^{p}-\mathrm{d} \varepsilon_{z z}^{p}\right)}{3}
\end{array}\right)
$$


onde os subscritos $x, y$ e $z$ representam os eixos ortogonais de ortotropia. Para as chapas laminadas estes eixos estão alinhados com as direções de laminação, transversal e com a espessura. Os coeficientes $c_{i=1 \sim 6}$ representam a anisotropia do material. O expoente $\zeta$ está associado à estrutura cristalina do material, e tem valores de 1 até $\infty$, e afeta a curvatura da superfície da deformação efetiva. Valores recomendados para este critério são de $\zeta=4 / 3$ para materiais $\mathrm{CFC}^{2}$ e $\zeta=3 / 2$ para materiais $\mathrm{CCC}^{3}$.

Para o estado plano de tensão $\mathbf{L}$ pode ser espresso

$$
\mathbf{L}=\left(\begin{array}{cc}
\frac{c_{3}\left(\mathrm{~d} \varepsilon_{x x}^{p}-\mathrm{d} \varepsilon_{y y}^{p}\right)-c_{2}\left(\mathrm{~d} \varepsilon_{z z}^{p}-\mathrm{d} \varepsilon_{x x}^{p}\right)}{3} & c_{6} \mathrm{~d} \varepsilon_{x y}^{p} \\
c_{6} \mathrm{~d} \varepsilon_{x y}^{p} & \frac{c_{1}\left(\mathrm{~d} \varepsilon_{y y}^{p}-\mathrm{d} \varepsilon_{z z}^{p}\right)-c_{3}\left(\mathrm{~d} \varepsilon_{x x}^{p}-\mathrm{d} \varepsilon_{y y}^{p}\right)}{3}
\end{array}\right)
$$

Os autovalores de (3.79) fornecem $\mathrm{d} \varepsilon_{1}, \mathrm{~d} \varepsilon_{2}$ e $\mathrm{d} \varepsilon_{3}$ vem da condição de incompressibilidade, $\mathrm{d} \varepsilon_{3}=-\left(\mathrm{d} \varepsilon_{1}+\mathrm{d} \varepsilon_{2}\right)$

De (3.79) temos os autovalores

$$
\begin{aligned}
& \mathrm{d} \varepsilon_{1}=\frac{1}{6}\left(c_{2}\left(2 \mathrm{~d} \varepsilon_{x x}+\mathrm{d} \varepsilon_{y y}\right)+c_{1}\left(\mathrm{~d} \varepsilon_{x x}+2 \mathrm{~d} \varepsilon_{y y}\right)+\right. \\
& \left.+\sqrt{36 c_{6}^{2} \mathrm{~d} \varepsilon_{x y}^{2}+\left(\left(c_{1}-2\left(c_{2}+c_{3}\right)\right) \mathrm{d} \varepsilon_{x x}+\left(2 c_{1}-c_{2}+2 c_{3}\right) \mathrm{d} \varepsilon_{y y}\right)^{2}}\right) \\
& \mathrm{d} \varepsilon_{2}=\frac{1}{6}\left(c_{2}\left(2 \mathrm{~d} \varepsilon_{x x}+\mathrm{d} \varepsilon_{y y}\right)+c_{1}\left(\mathrm{~d} \varepsilon_{x x}+2 \mathrm{~d} \varepsilon_{y y}\right)-\right. \\
& \left.-\sqrt{36 c_{6}^{2} \mathrm{~d} \varepsilon_{x y}^{2}+\left(\left(c_{1}-2\left(c_{2}+c_{3}\right)\right) \mathrm{d} \varepsilon_{x x}+\left(2 c_{1}-c_{2}+2 c_{3}\right) \mathrm{d} \varepsilon_{y y}\right)^{2}}\right)
\end{aligned}
$$

onde $\mathrm{d} \varepsilon_{z z}=-\left(\mathrm{d} \varepsilon_{x x}+\mathrm{d} \varepsilon_{y y}\right)$

Substituindo (3.80) em (3.76) temos

$$
\begin{aligned}
\mathrm{d} \bar{\varepsilon} & =\frac{1}{k}\left(2 ^ { - 1 - \zeta } 3 ^ { - \zeta } \left(2^{\zeta}\left|c_{2}\left(2 \mathrm{~d} \varepsilon_{x x}+\mathrm{d} \varepsilon_{y y}\right)+c_{1}\left(\mathrm{~d} \varepsilon_{x x}+2 \mathrm{~d} \varepsilon_{y y}\right)\right|^{\zeta}+\right.\right. \\
& +\mid c_{2}\left(2 \mathrm{~d} \varepsilon_{x x}+\mathrm{d} \varepsilon_{y y}\right)+c_{1}\left(\mathrm{~d} \varepsilon_{x x}+2 \mathrm{~d} \varepsilon_{y y}\right)+ \\
& +\left.\sqrt{36 c_{6}^{2} \mathrm{~d} \varepsilon_{x y}^{2}+\left(\left(c_{1}-2\left(c_{2}+c_{3}\right)\right) \mathrm{d} \varepsilon_{x x}+\left(2 c_{1}-c_{2}+2 c_{3}\right) \mathrm{d} \varepsilon_{y y}\right)^{2}}\right|^{\zeta}+ \\
& +\mid c_{2}\left(2 \mathrm{~d} \varepsilon_{x x}+\mathrm{d} \varepsilon_{y y}\right)+c_{1}\left(\mathrm{~d} \varepsilon_{x x}+2 \mathrm{~d} \varepsilon_{y y}\right)- \\
& \left.\left.-\left.\sqrt{36 c_{6}^{2} \mathrm{~d} \varepsilon_{x y}^{2}+\left(\left(c_{1}-2\left(c_{2}+c_{3}\right)\right) \mathrm{d} \varepsilon_{x x}+\left(2 c_{1}-c_{2}+2 c_{3}\right) \mathrm{d} \varepsilon_{y y}\right)^{2}}\right|^{\zeta}\right)\right)^{1 / \zeta}
\end{aligned}
$$

\footnotetext{
${ }^{2}$ cúbico de face centrada

${ }^{3}$ cúbico de corpo centrado
} 
em forma matricial

$$
\mathrm{d} \bar{\varepsilon}=\frac{1}{k}\left(2^{-1-\zeta} 3^{-\zeta}\left(2^{\zeta}\left|\mathbf{D}_{\mathrm{B} \mathbf{1}} \mathrm{d} \varepsilon\right|^{\zeta}+\left|\mathbf{D}_{\mathrm{B} \mathbf{1}} \mathrm{d} \boldsymbol{\varepsilon}+\sqrt{\mathrm{d} \varepsilon^{T} \mathbf{D}_{\mathbf{B}} \mathrm{d} \varepsilon}\right|^{\zeta}+\left|\mathbf{D}_{\mathrm{B} \mathbf{1}} \boldsymbol{\varepsilon}-\sqrt{\mathrm{d} \varepsilon^{T} \mathbf{D}_{\mathbf{B}} \mathrm{d} \varepsilon}\right|^{\zeta}\right)\right)^{1 / \zeta}
$$

com

$$
\mathrm{d} \varepsilon^{T}=\left[\begin{array}{lll}
\mathrm{d} \varepsilon_{x x} & \mathrm{~d} \varepsilon_{y y} & \mathrm{~d} \varepsilon_{x y}
\end{array}\right]
$$

e

$$
\begin{aligned}
& \mathbf{D}_{\mathbf{B}}=\left(\begin{array}{ccc}
a_{11} & a_{12} & a_{13} \\
a_{12} & a_{22} & a_{23} \\
a_{13} & a_{23} & a_{33}
\end{array}\right) \\
& a_{11}=c_{1}^{2}-4 c_{1} c_{2}+4 c_{2}^{2}-4 c_{1} c_{3}+8 c_{2} c_{3}+4 c_{3}^{2} \\
& a_{12}=\left(4 c_{1}^{2}-10 c_{1} c_{2}+4 c_{2}^{2}-4 c_{1} c_{3}-4 c_{2} c_{3}-8 c_{3}^{2}\right) / 2 \\
& a_{13}=0 \\
& a_{22}=4 c_{1}^{2}-4 c_{1} c_{2}+c_{2}^{2}+8 c_{1} c_{3}-4 c_{2} c_{3}+4 c_{3}^{2} \\
& a_{23}=0 \\
& a_{33}=36 c_{6}^{2}
\end{aligned}
$$

e

$$
\mathbf{D}_{\mathbf{B} 1}=\left(\begin{array}{c}
c_{1}+2 c_{2} \\
2 c_{1}+c_{2} \\
0
\end{array}\right)
$$

\subsection{Equação Constitutiva para a Curva Tensão De- formação de Materiais Rígido-plásticos}

As duas equações mais usadas para descrever as curvas de tensão-deformação na análise da conformação plástica em metais são:

Modelo de endurecimento exponencial:

$$
\bar{\sigma}=K \bar{\varepsilon}^{n}
$$


onde $K$ é uma constante e $n$, o coeficiente de endurecimento pela deformação. $\bar{\sigma}$ e $\bar{\varepsilon}$ são a tensão e deformação efetivas verdadeiras, respectivamente.

\section{Equação de Swift:}

$$
\bar{\sigma}=A(B+\bar{\varepsilon})^{n}
$$

onde $0 \leq n \leq 1$ e $A, B, n$ são constantes para um dado material.

As constantes de ambas as equações são determinadas por meio de ensaios com espécimes reais. Neste estudo, a equação constitutiva para a curva tensão deformação é baseada na consideração da dissipação de energia. Na abordagem para grandes deformações, $\bar{S}$, a atual tensão efetiva (Piola-Kirchoff, PK-2), tem a forma:

$$
\bar{S}=\bar{S}_{0}+H_{0}(\mathrm{~d} \bar{E})
$$

onde $\bar{S}_{0}$ é a tensão efetiva e $H_{0}=\mathrm{d} \bar{S} / \mathrm{d} \bar{E}$, ambos avaliados em $t=t_{0}$. Através da análise de uma curva tensão-deformação mostrada na figura 8, pode-se facilmente estabelecer uma relação entre $\mathrm{d} \bar{\sigma} / \mathrm{d} \bar{\varepsilon}$ e $\mathrm{d} \bar{S} / \mathrm{d} \bar{E}$. Assumindo que durante a deformação, a dissipação de energia seja a mesma em cada incremento, pode-se escrever

$$
\int_{\bar{\varepsilon}_{0}}^{\bar{\varepsilon}} \bar{\sigma} \mathrm{d} \bar{\varepsilon}=\int_{\bar{E}_{0}}^{\bar{E}} \bar{S} \mathrm{~d} \bar{E}
$$

ou, aproximadamente

$$
\left(\bar{\sigma}+\bar{\sigma}_{0}\right)(\mathrm{d} \bar{\varepsilon})=\left(\bar{S}+\bar{S}_{0}\right)(\mathrm{d} \bar{E})
$$

substituindo $\bar{S}_{0}=\bar{\sigma}_{0}$ e re-arranjando, obtém-se

$$
\bar{S}=\left(\frac{\bar{\varepsilon}}{\mathrm{d} \bar{E}}-1\right) \bar{\sigma}_{0}+\left(\frac{\bar{\varepsilon}}{\mathrm{d} \bar{E}}\right) \bar{\sigma}
$$




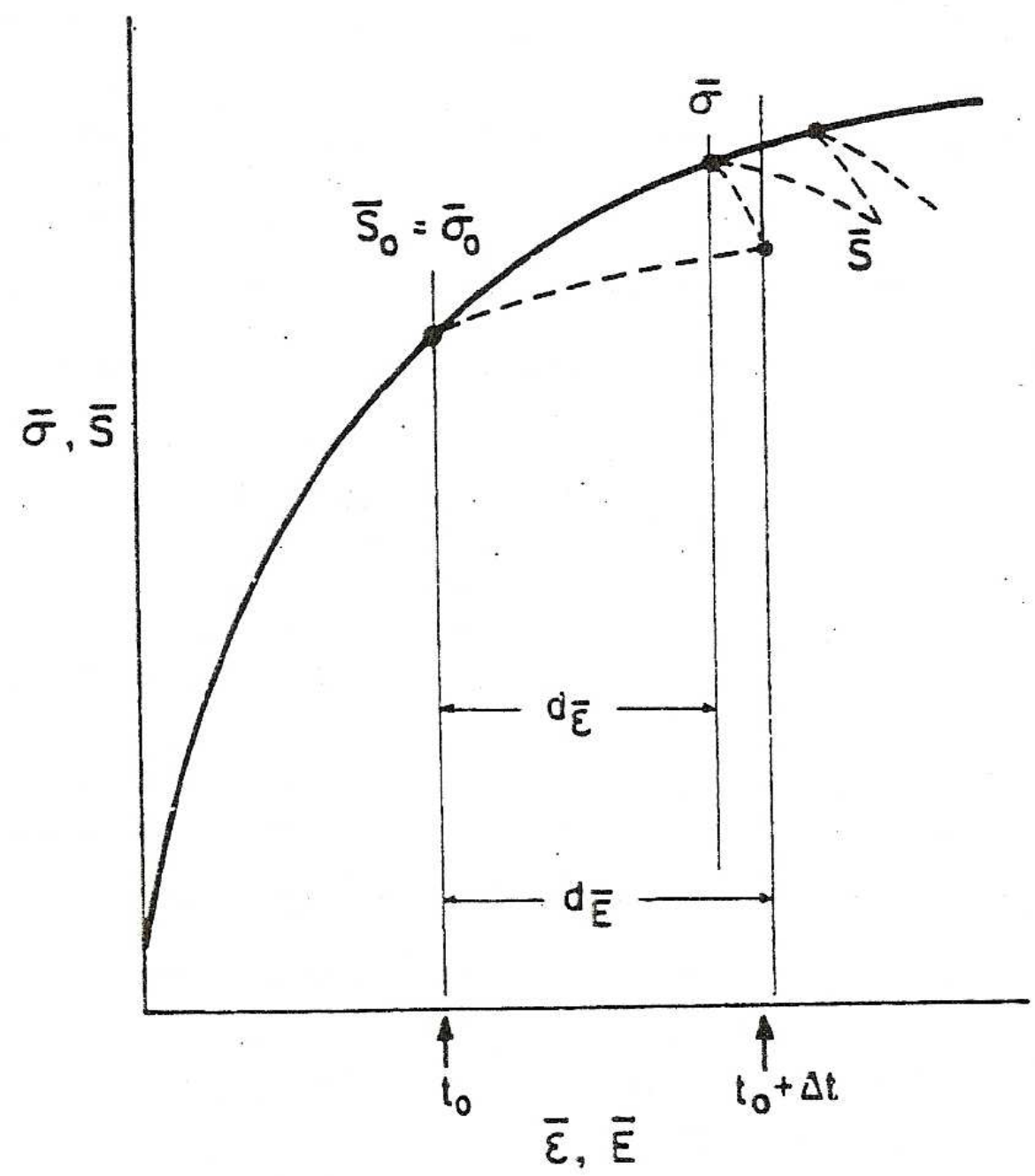

Figura 8: Curva teórica de tensão-deformação. Toh (1983)

Expandindo em séries de Taylor, e negligenciando os termos de maior ordem,

$$
\begin{aligned}
& \bar{S}=\bar{S}_{0}+H_{0}(\mathrm{~d} \bar{E}) \\
& \bar{\sigma}=\bar{\sigma}_{0}+h_{0}(\mathrm{~d} \bar{\varepsilon})
\end{aligned}
$$

onde $h_{0}=\mathrm{d} \bar{\sigma} / \mathrm{d} \bar{\varepsilon}$ é avaliado em $t=t_{0}$. Com a substituição da equação 3.93 em 3.92:

$$
H_{0}=\left(\frac{\mathrm{d} \bar{\varepsilon}}{\mathrm{d} \bar{E}}\right)^{2} h_{0}+2\left(\frac{\mathrm{d} \bar{\varepsilon}-\mathrm{d} \bar{E}}{\mathrm{~d} \bar{E}^{2}}\right) \bar{\sigma}_{0}
$$

Como o incremento de deformação efetiva (verdadeira) na forma

$$
\mathrm{d} \bar{\varepsilon}=\ln \left(1+\frac{\mathrm{d} l}{l_{0}}\right)=\frac{\mathrm{d} l}{l_{0}}-\frac{1}{2}\left(\frac{\mathrm{d} l}{l_{0}}\right)^{2}+\cdots
$$


e o incremento de deformação efetiva Lagrangeana

$$
\mathrm{d} \bar{E}=\frac{\mathrm{d} l}{l_{0}}+\frac{1}{2}\left(\frac{\mathrm{d} l}{l_{0}}\right)^{2}
$$

onde $\mathrm{d} l / l_{0}$ é a deformação nominal ou da engenharia no ensaio de tração uniaxial. Assim, a equação 3.94 pode ser escrita na forma:

$$
H_{0}=h_{0}-2 \bar{\sigma}_{0}
$$

com a substituição de 3.97 em 3.93 chega-se a

$$
\bar{S}=\bar{\sigma}_{0}+\left(h_{0}-2 \bar{\sigma}_{0}\right) \mathrm{d} \bar{E}
$$

Esta é a equação tensão-deformação que é usada para a implementação computacional.

\subsection{Zona Rígida}

Devido à geometria de alguns processos de estampagem, podem existir partes do material com comportamento quase rígido. Esta parte do material é caracterizada por ter um valor de deformação efetiva extremamente pequeno em comparação ao resto do corpo em deformação. Isto acontece em operações com punção de fundo plano, onde os materiais em torno da região plana deformam menos do que os materiais fora dessa região, criando uma zona em que o material está no regime elástico ou quase rígido, comparado com as outras regiões.

Para eliminar a zona rígida do cálculo numérico, e para evitar as dificuldades em determinar a zona rígida, um método de offset da deformação efetiva Chen (1978) é usada. Em cada deformação incremental que o elemento possuir deformações efetivas menores que um valor limite, $\mathrm{d} \bar{E}_{0}$, a tensão para estes elementos é assumida como variando linearmente neste passo incremental (como material Hookeano). 


\section{Mecânica da Deformação de chapas}

\subsection{Deformação da Chapa no Estado Plano de Tensão}

Se uma chapa de espessura inicial $t_{0}$ é marcada com uma grade de círculos de diâmetro $d_{0}$ ou com uma malha quadrada de tamanho $d_{0}$ como na figura 9 a, então durante uma deformação uniforme, os círculos deformarão em elipses com eixos $d_{1}$ e $d_{2}$ respectivamente. Se a malha quadrada está alinhada com as direções principais, ela se tornará retangular (figura $9 \mathrm{~b}$ ). No instante mostrado na figura $9 \mathrm{~b}$, a espessura é $t$ e as tensões são $\sigma_{1}$ e $\sigma_{2}$.

\subsubsection{Distribuição de Deformações}

No estudo de qualquer processo de conformação, geralmente é primeiramente determinada a deformação sobre uma parte. Isto pode ser feito medindo-se a grade de círculos da figura 9. Usando como exemplo a estampagem profunda de um copo cilíndrico (figura 10 a), devido a simetria em relação ao eixo, podem-se considerar as deformações sobre os pontos na linha mostrada na figura 10 b. Plotando estas deformações no espaço de deformações principais (figura 10 c), tem-se a curva de deformações para um estágio particular do processo. Conforme o processo continua, a curva se expande, não necessariamente de forma uniforme, alguns pontos podem parar de deformar e outros podem atingir o limite do processo. Para cada processo existirá uma curva de deformação como mostrado na figura 10 c e muitas informações podem ser obtidas deste diagrama. 


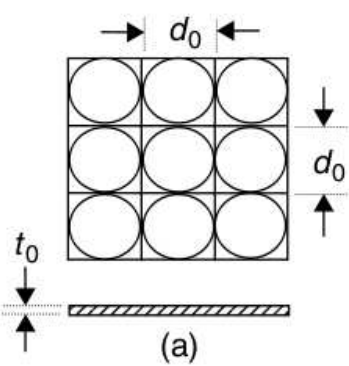

(a)

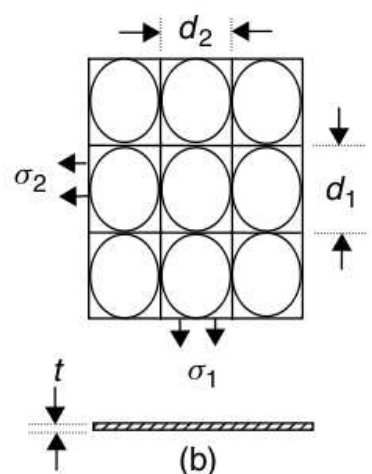

(b)

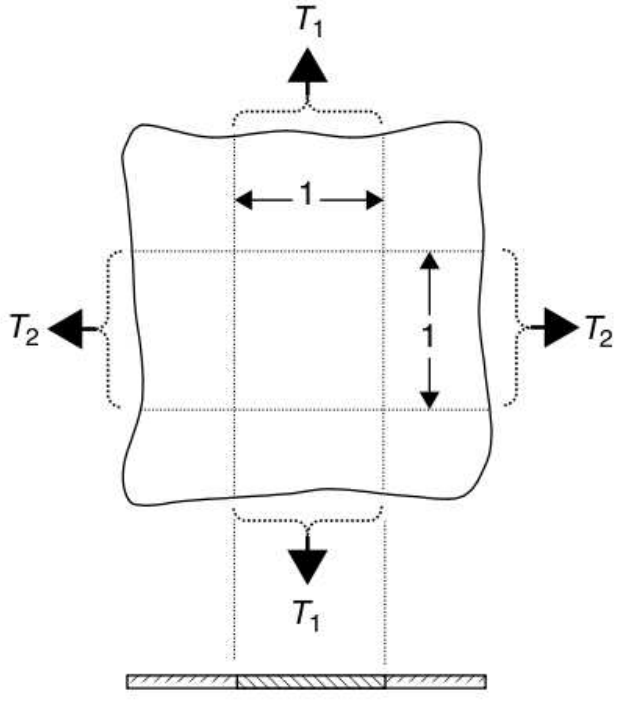

(c)

Figura 9: Um elemento de chapa mostrando: a) estado inicial com uma grade de círculos e quadrados marcados, b) estado deformado com os círculos deformados em elipses com os eixos $d_{1}$ e $d_{2}$ e c) as trações aplicadas (Marciniak et al. (2002)).

\subsubsection{Diagrama de Deformação}

As deformações dos pontos da figura 10 c podem ser obtidos medindo-se as grades de círculos da figura 9. Assim pode-se obter as deformações principais

$$
\varepsilon_{1}=\ln \frac{d_{1}}{d_{0}} ; \varepsilon_{2}=\ln \frac{d_{2}}{d_{0}} ; \varepsilon_{3}=\ln \frac{t}{t_{0}}
$$

Assumindo que o caminho de deformação é linear, a razão de deformação é constante e dada por

$$
\beta=\frac{\varepsilon_{2}}{\varepsilon_{1}}=\frac{\ln \frac{d_{2}}{d_{0}}}{\ln \frac{d_{1}}{d_{0}}}
$$

Na equação 4.1, a deformação na espessura é determinada medindo-se a espessura inicial e final, ela também pode ser obtida das deformações principais $\varepsilon_{1}$ e $\varepsilon_{2}$, assumindose volume constante

$$
\varepsilon_{3}=\ln \frac{t}{t_{0}}=-(1+\beta) \varepsilon_{1}=-(1+\beta) \ln \frac{d_{1}}{d_{0}}
$$




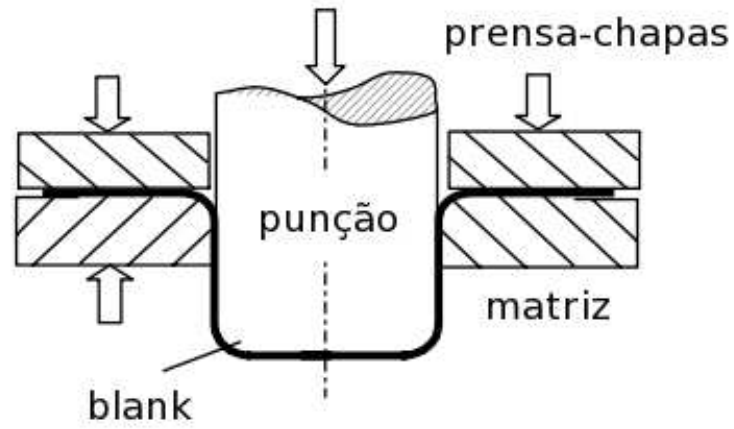

(a)

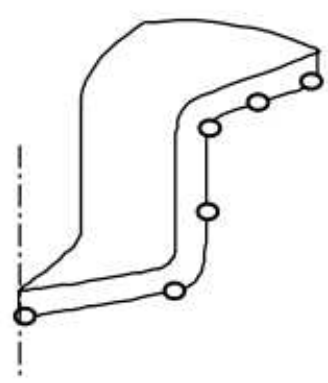

(b)

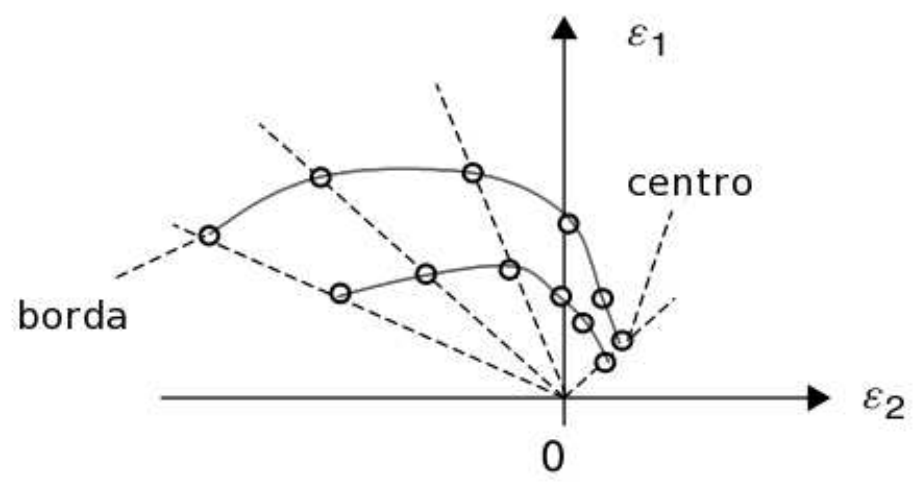

(c)

Figura 10: a) Estampagem profunda de um copo circular, b) seção do copo mostrando a localização das deformações medidas e c) deformações plotadas para dois estágios do processo de estampagem (Marciniak et al. (2002)).

Das equações acima, pode-se escrever as deformações principais de um modo mais conveniente

$$
\varepsilon_{1}=\ln \frac{d_{1}}{d_{0}} ; \varepsilon_{2}=\ln \frac{d_{2}}{d_{0}}=\beta \varepsilon_{1} ; \varepsilon_{3}=\ln \frac{t}{t_{0}}=-(1+\beta) \varepsilon_{1}
$$

onde $\beta$ é uma constante.

\subsubsection{Modos de Deformação}

Pode-se examinar com mais detalhes as características dos diferentes caminhos de deformação na figura 11. Este diagrama não representa nenhum processo em particular, mas será usado para discutir os diferentes modos de deformação. A elipse mostra o contorno para uma mesma deformação efetiva $\bar{\varepsilon}$. 


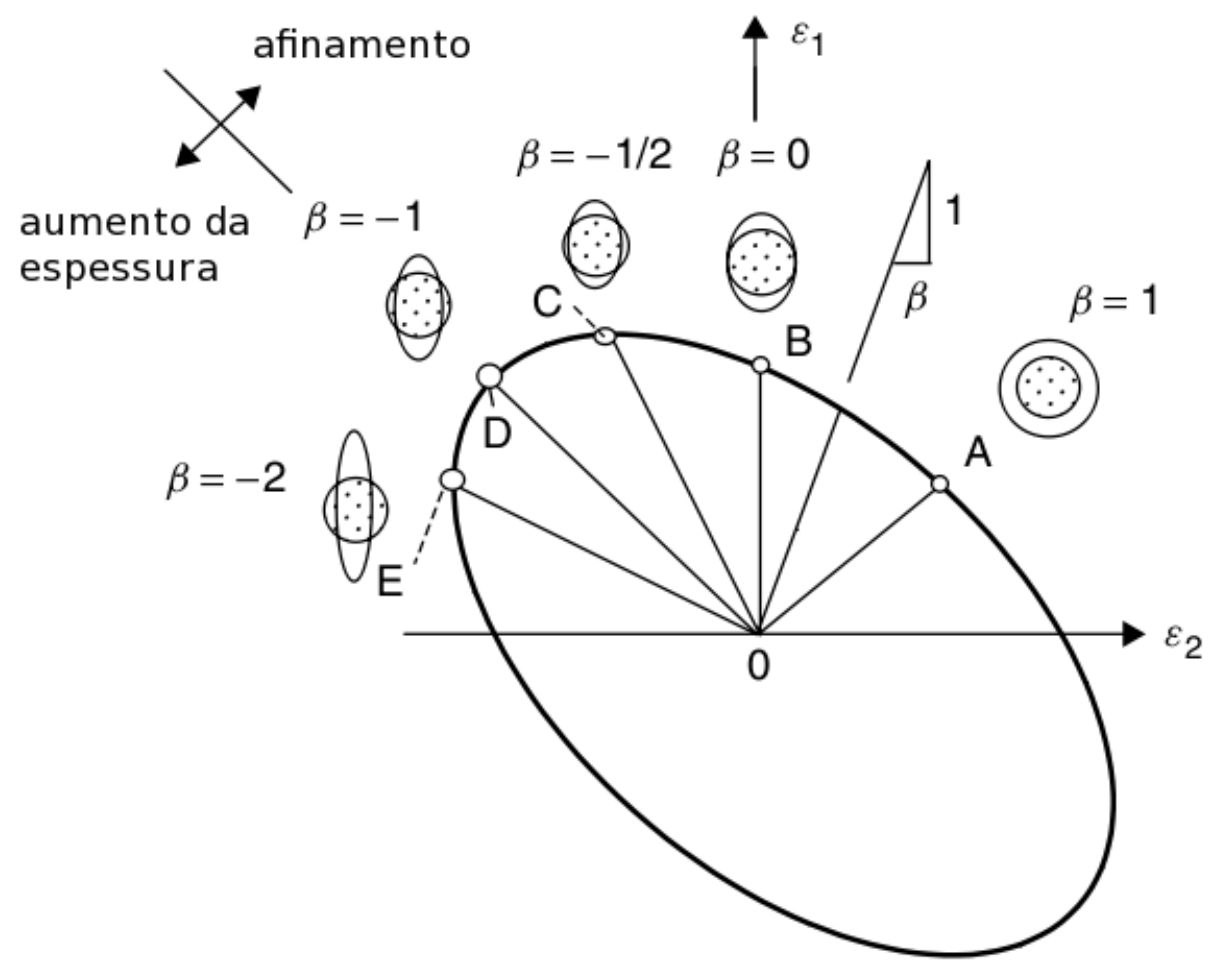

Figura 11: O diagrama de deformação mostrando os diferentes modos de deformação correspondendo a diferentes razões de deformação (Marciniak et al. (2002)).

Estiramento equibiaxial, $\beta=1$

O caminho 0A (figura 11) indica o estiramento equibiaxial. Uma chapa estirada com um punção hemisférico deformará deste modo no centro do processo, como mostrado na figura 12. A deformação é igual em todas as direções e o círculo da grade expandirá, mantendo-se circular. Como $\beta=1$, a deformação na espessura é $\varepsilon_{3}=-2 \varepsilon_{1}$.

Estado plano de deformação, $\beta=0$

Neste processo ilustrado pelo caminho 0B (figura 11), a chapa se espande apenas em uma direção e o círculo se torna uma elipse no qual o eixo menor não deforma. Este tipo de deformação é observado em paredes como na figura 13.

Tração uniaxial, $\beta=-\frac{1}{2}$

O ponto $\mathrm{C}$ na figura 11 é o processo em um teste de tração uniaxial e ocorre na 


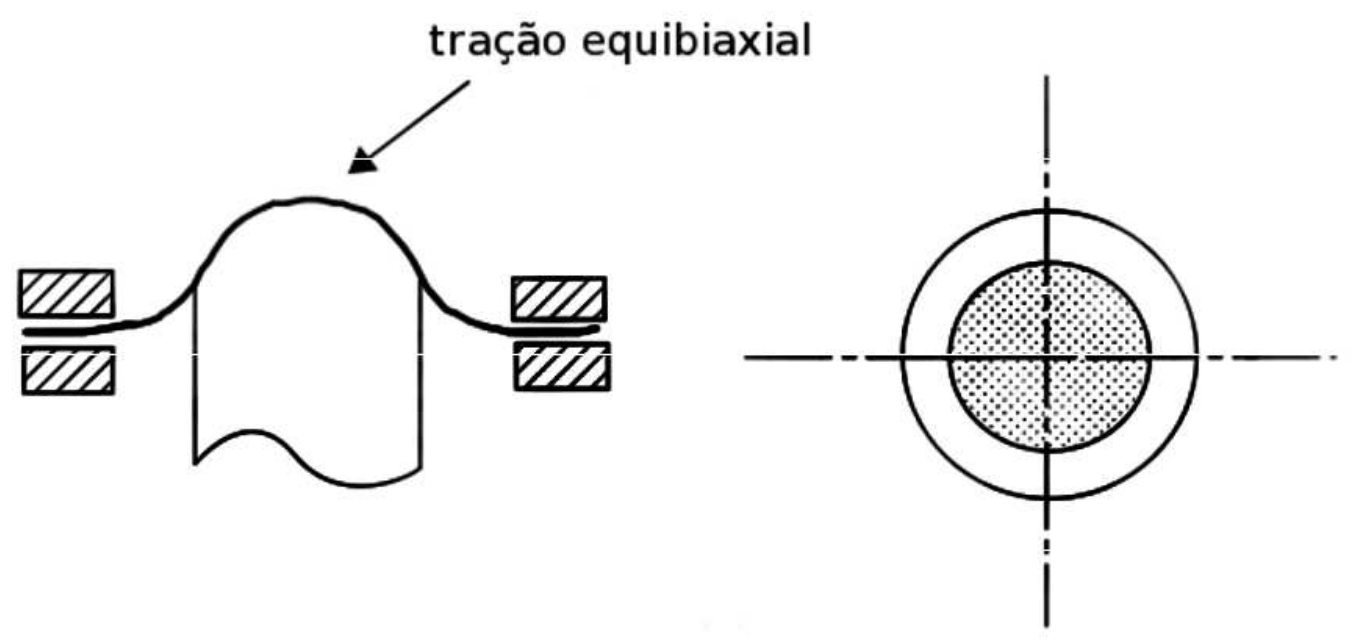

Figura 12: Estiramento equibiaxial no centro da chapa (Marciniak et al. (2002)).

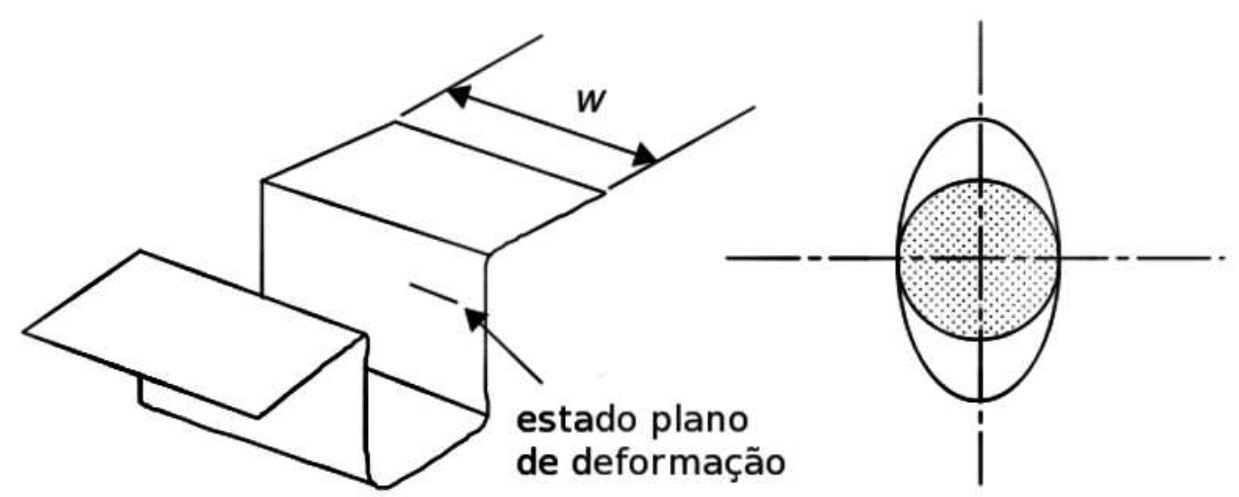

Figura 13: Deformação no estado plano de deformação em uma parede de uma chapa comprida (Marciniak et al. (2002)).

chapa quando $\sigma_{2}=0$. A chapa estira em uma direção e contrai na outra. Este processo acontecerá quando uma borda livre é estirada, como no caso da expansão de um furo (figura 14).

Espessura constante ou repuxo, $\beta=-1$

Neste processo, ponto D, as tensões e deformações são iguais e opostas e a chapa deforma sem mudança de espessura. É chamado de repuxo e é observado quando a chapa é empurrada em uma matriz, como na flange de uma estampagem profunda (figura 15). O processo também é chamado de cisalhamento puro.

Compressão uniaxial, $\beta=-2$ 

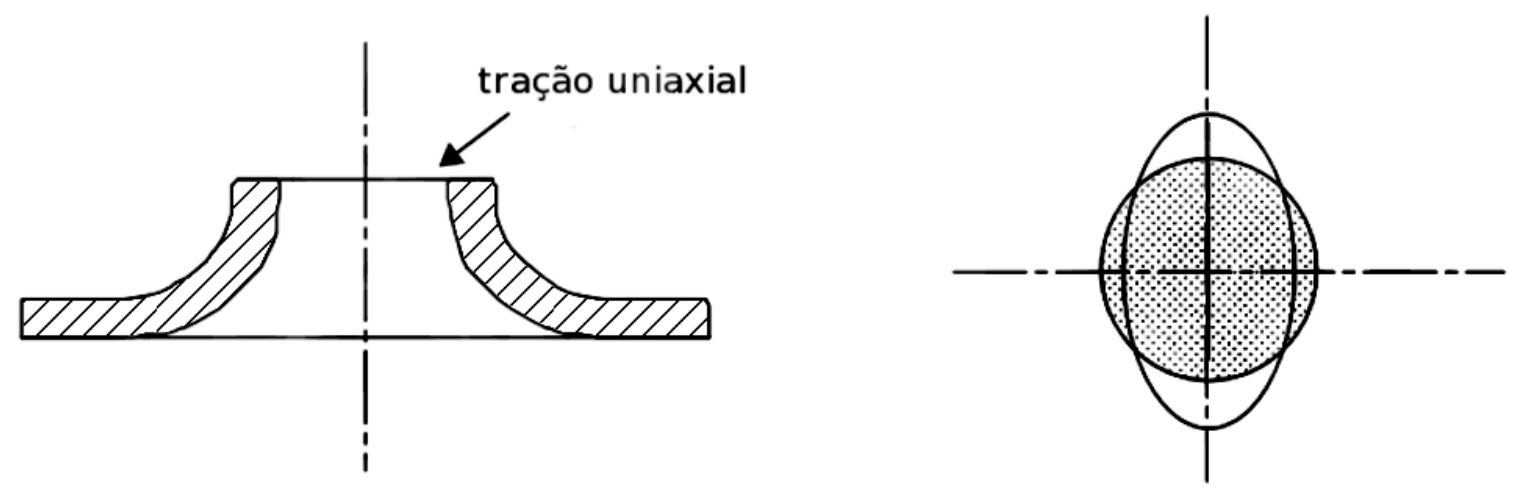

Figura 14: Tração uniaxial em um furo expandido (Marciniak et al. (2002)).
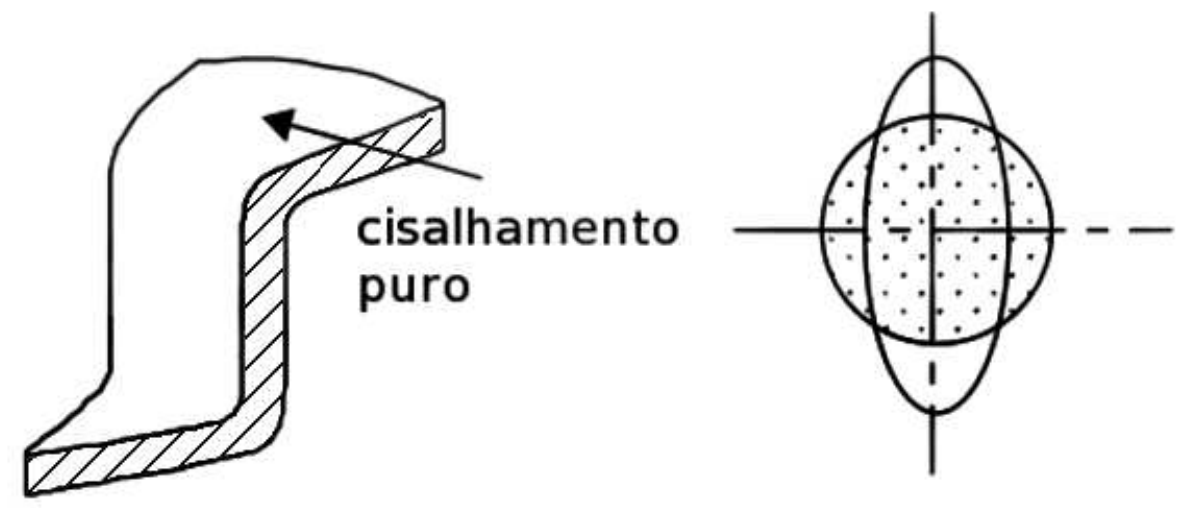

Figura 15: Repuxo ou cisalhamento puro na flange de uma estampagem profunda de copo cilindrico (Marciniak et al. (2002)).

Este processo, indicado pelo ponto E, é um caso extremo e ocorre quando a tensão principal $\sigma_{1}$ é zero, como na borda da chapa em uma estampagem profunda (figura 16). A tensão principal $\sigma_{2}$ é compressiva, isto é, $\sigma_{2}=-\bar{\sigma}$. Neste processo a chapa aumenta a espessura.

\subsubsection{Diagrama de tensão}

Um diagrama de tensão, onde os pontos são associados a um ponto de deformação, é útil para o entendimento das forças envolvidas nos processos de deformação. A figura 17 mostra um exemplo de diagrama de tensão, não representando um processo particular. Nele são mostradas curvas para tensão efetiva iguais, que são curvas de escoamento para uma dada tensão de escoamento $\left(\sigma_{e}\right)$. Durante a deformação, o escoamento plástico 


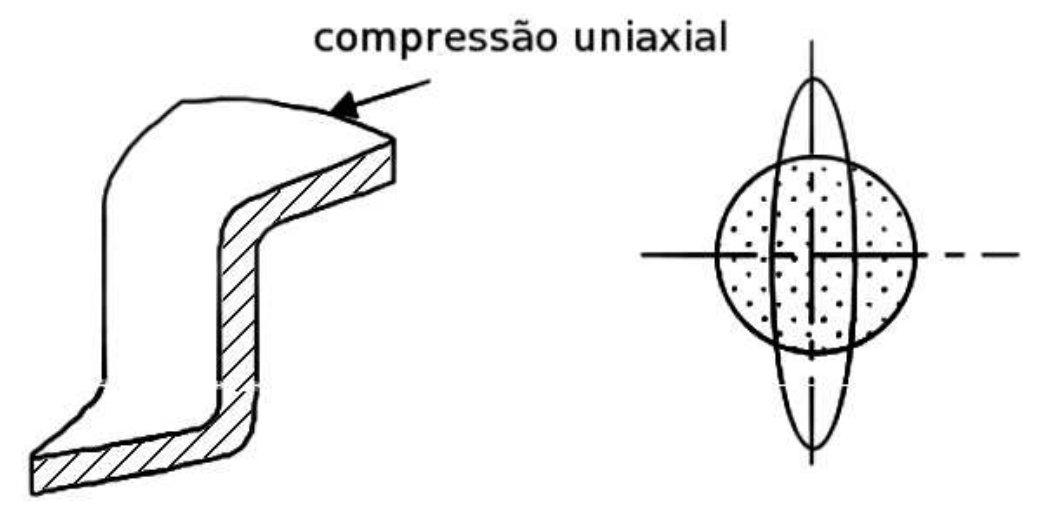

Figura 16: Compressão uniaxial na borda da estampagem profunda (Marciniak et al. (2002)).

iniciará na curva de escoamento indicada pela linha contínua, quando $\bar{\sigma}=\left(\sigma_{e}\right)_{0}$ e o caminho do carregamento será ao longo da linha radial de inclinação $1 / \alpha$. O estado de tensão atual é mostrado como linha tracejada.

As tensões principais são

$$
\sigma_{1} ; \quad \sigma_{2}=\alpha \sigma_{1} \quad \text { e } \quad \sigma_{3}=0
$$

e cada caminho no diagrama de deformação, figura 11, tem um correspondente no diagrama de tensão, listado abaixo.

Estiramento equibiaxial, $\alpha=\beta=1$

No ponto A, a chapa é estirada em tração equibiaxial e

$$
\sigma_{1}=\sigma_{2}=\bar{\sigma}
$$

Estado plano de deformação, $\alpha=1 / 2, \beta=0$

Para o estado plano de deformação, isto é, deformação zero na direção $2\left(\varepsilon_{2}=0\right)$, o estado de tensão é indicado pelo ponto B e

$$
\sigma_{1}=\frac{2}{\sqrt{3}} \bar{\sigma} \quad \text { e } \quad \sigma_{2}=\frac{1}{2} \sigma_{1}
$$




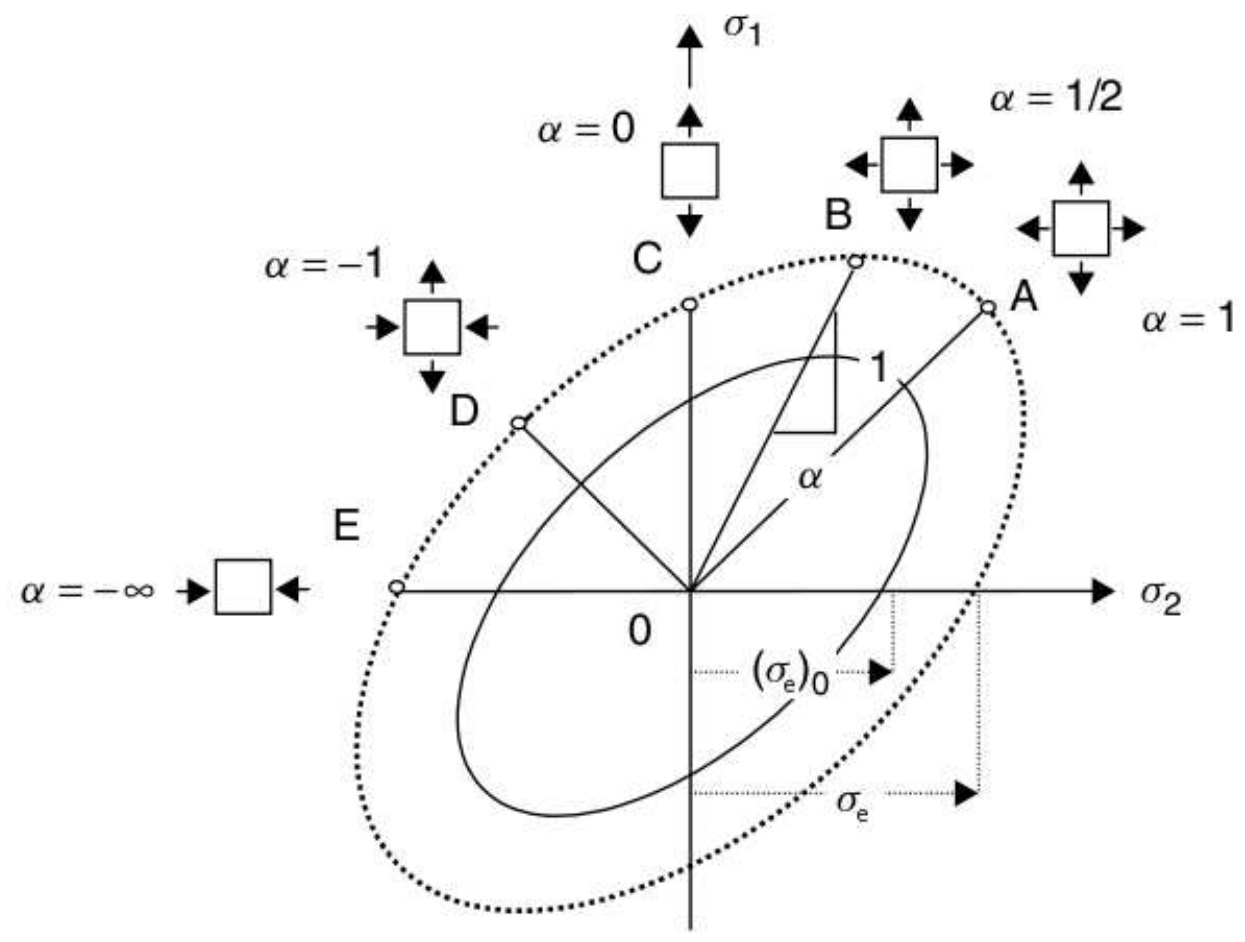

Figura 17: O processo mostrado na figura 10 representado no espaço de tensões (a elipse de escoamento representada em linha tracejada) (Marciniak et al. (2002)).

Tração uniaxial, $\alpha=0, \beta=-\frac{1}{2}$

Neste processo a tensão principal $\sigma_{1}$ é igual a tensão de escoamento $\sigma_{e}$ e a tensão principal $\sigma_{2}$ é igual a zero. Ela ocorre nos testes de tração uniaxial.

Espessura constante ou repuxo, $\alpha=-1, \beta=-1$

Ao longo da diagonal à esquerda em $\mathrm{D}$, as tensões e deformações são iguais e opostas e não há mudança na espessura. As tensões são

$$
\sigma_{1}=\frac{1}{\sqrt{3}} \sigma_{e}=0,58 \sigma_{e}=0,58 \bar{\sigma}
$$

$\mathrm{e}$

$$
\sigma_{2}=-\frac{1}{\sqrt{3}} \sigma_{e}=-0,58 \sigma_{e}=-0,58 \bar{\sigma}
$$

Pode-se notar que as magnitudes de tensão que causam a deformação são mínimas, elas são $58 \%$ da tensão requirida para escoar um elemento similar em tração simples. Este pode ser considerado um modo de deformação ideal, pois as tensões são baixas e não há 
mudança na espessura.

Compressão uniaxial, $\alpha=-\infty, \beta=-2$

Este modo ocorre geralmente nas bordas livres na estampagem profunda, sendo a tensão neste local igual a zero. A tensão principal $\sigma_{2}$ é igual a tensão de escoamento de compressão

$$
\sigma_{1}=0 \quad \text { e } \quad \sigma_{2}=-\sigma_{e}=\bar{\sigma}
$$

\subsection{Estudo do Dobramento}

O estudo do dobramento de chapas metálicas remete inicialmente à resistência dos materiais e ao estudo da flexão pura. Neste tipo de conformação, dois pontos são de particular interesse para o controle do processo: a possibilidade de ruptura e o controle da forma final. O raio de dobramento e a ductilidade são importantes para o controle da possibilidade de rasgamento, enquanto a forma final é afetada pelo retorno elástico, dependente da propriedade elástica do material. O processo de conformação ocorre pela aplicação de um momento ou uma tensão sobre uma chapa apoiada em uma matriz, ou pela combinação de tensão e momento.

No estudo de chapas finas, seções transversais permanecem planas no dobramento, como ilustrado na figura 18. Também é considerado que as direções das tensões e deformações coincidem com a direção radial e circunferencial, de modo que não exista cisalhamento no plano radial e que os gradientes de tensão e deformação sejam zero na direção circunferencial.

Considerando uma fibra $A_{0} B_{0}$ situada na linha neutra, seu comprimento permanece inalterado após a dobra e pode ser expresso como:

$$
l_{S}=\theta \cdot \rho
$$

Uma fibra AB afastada de y da linha neutra, terá seu comprimento após a dobra definido 


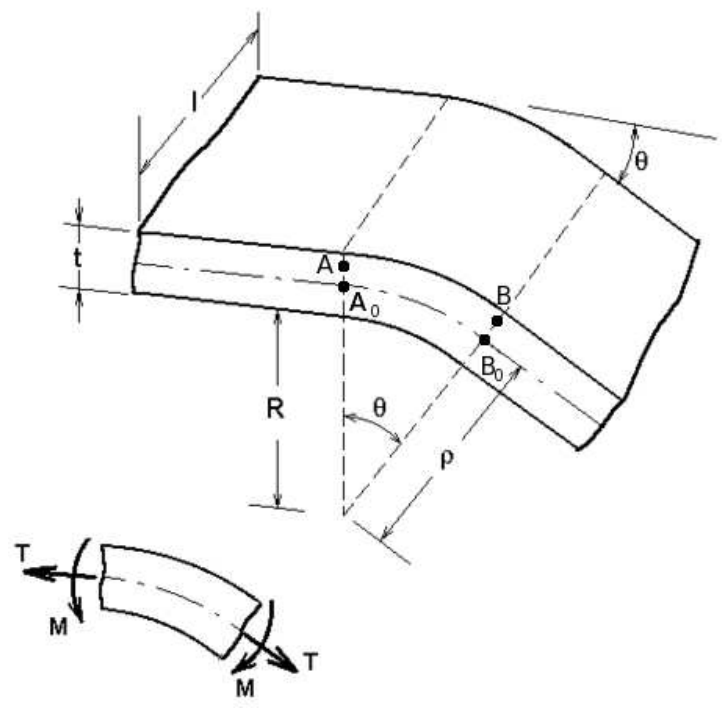

Figura 18: Variáveis do dobramento (Marciniak e Duncan (1992)).

por

$$
l=\theta(\rho+y)
$$

então

$$
l=l_{S}\left(1+\frac{y}{\rho}\right)
$$

A deformação axial da fibra $\mathrm{AB}$ é

$$
\varepsilon_{l}=\ln \left\{\left(l / l_{0}\right)[1+(y / \rho)]\right\}
$$

$\mathrm{Ou}$

$$
\varepsilon_{l}=\ln \left(l_{s} / l_{0}\right)+\ln [1+(y / \rho)]
$$

e podem ser identificadas duas componentes de deformação: uma associada à extensão da linha neutra, que será chamada de $\varepsilon_{a}$

$$
\varepsilon_{a}=\ln \left(l_{s} / l_{0}\right)
$$

e a componente de deformação por dobramento, $\varepsilon_{b}$

$$
\varepsilon_{b}=\ln [1+(y / \rho)]
$$




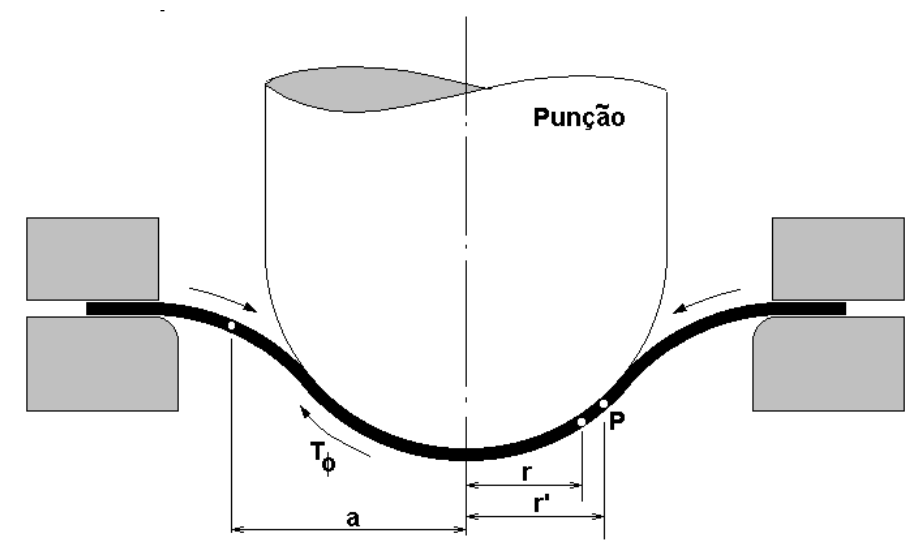

Figura 19: Conformação de um blank circular (Marciniak e Duncan (1992)).

então

$$
\varepsilon_{l}=\varepsilon_{a}+\varepsilon_{b}
$$

\subsection{Estudo do Estiramento}

Em um processo de conformação de uma chapa circular, como mostrado na figura 19, a chapa é estirada pelo punção por forças de tração meridianas, $\mathbf{T}_{\phi}$, que surgem da resistência à deformação plástica das regiões adjacentes. Se a posição de um ponto $P$ originalmente no raio $r$ na chapa, é dada por $r^{\prime}$, então a chapa sofreu um estiramento e $r^{\prime}>r$. Na região de deformação, há um círculo neutro de raio a, imutável, que divide a chapa em uma região de estampagem apenas, com espessura constante $(r>a)$ e outra de estiramento $(r<a)$. Neste estudo, será abordada a conformação de um disco fixo a partir do perímetro de uma circunferência de raio $a$.

\subsubsection{Estiramento de um Diafragma Circular}

Considerando a conformação de uma chapa circular em diafragma circular, através de uma pressão exercida por um fluido, o resultado pode ser descrito como uma superfície de revolução gerada pela rotação de uma curva, como pode ser visto na figura 20 .

Inicialmente será feito um estudo da distribuição de deformações. A partir da figura 21, pode-se afirmar que em um ponto $P$, de raio $r^{\prime}$, as deformações são: 


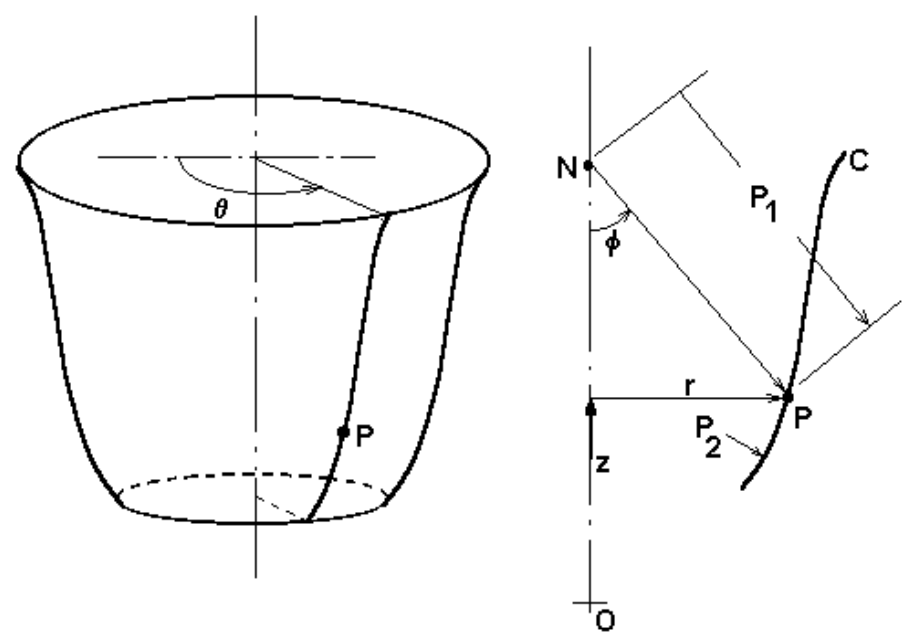

Figura 20: Superfície de revolução gerada pela revolução da curva $C$ (Marciniak e Duncan (1992)).

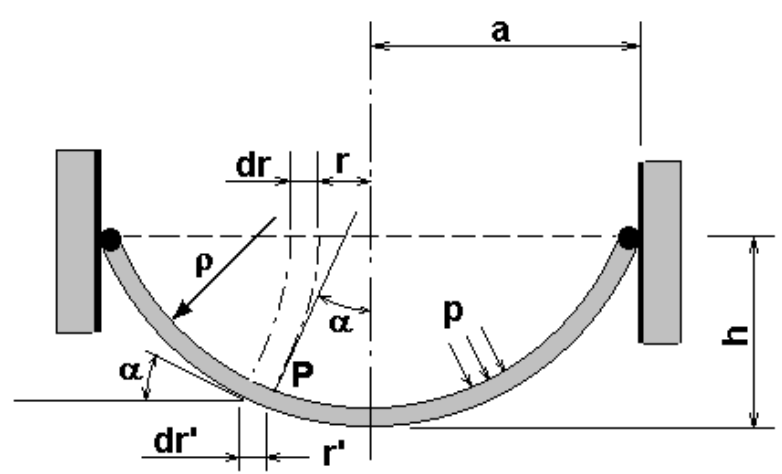

Figura 21: Conformando uma chapa fina com pressão de fluido (Marciniak e Duncan (1992)).

$$
\varepsilon_{\theta}=\ln \left(r^{\prime} / r\right) ; \quad \varepsilon_{\phi}=\ln \left[\mathrm{d} r^{\prime} / \mathrm{d} r \cdot \cos \alpha\right]
$$

onde r é o raio inicial do ponto. Por simetria, estas deformações são iguais no pólo. Nas bordas da chapa, em uma circunferência de raio $a$, a deformação $\varepsilon_{\theta}$ é zero.Existe também, um ponto intermediário em que um estado de deformação bi-axial é igual:

$$
\varepsilon_{\theta}=\varepsilon_{\phi}=-\frac{1}{2} \varepsilon_{i}
$$

da equação 4.19,

$$
\frac{\mathrm{d} r}{r}=\frac{\mathrm{d} r^{\prime}}{r^{\prime} \cos \alpha}=\frac{\rho \mathrm{d} r^{\prime}}{r^{\prime}\left(\rho^{2}-r^{\prime 2}\right)^{1 / 2}}
$$


onde,

$$
\cos \alpha=\left[1-\left(\frac{r^{\prime}}{\rho}\right)^{2}\right]^{1 / 2}
$$

e $\rho$ é o raio de curvatura da casca.

Integrando a equação 4.21, obtém-se:

$$
c_{1} \ln r=c_{2} \ln \left(\frac{r^{\prime}}{\rho+\left(\rho^{2}-r^{\prime 2}\right)^{1 / 2}}\right)
$$

Fazendo $r=r^{\prime}=a$,

$$
\begin{gathered}
r=r^{\prime} \frac{\rho^{\prime}+\left(\rho^{2}-a^{2}\right)^{1 / 2}}{\rho+\left(\rho^{2}-r^{\prime 2}\right)^{1 / 2}} \\
\varepsilon_{\theta}=\varepsilon_{\phi}=\ln \left(r^{\prime} / r\right)=\ln \left(\frac{\rho+\left(\rho^{2}-r^{\prime 2}\right)^{1 / 2}}{\rho+\left(\rho^{2}-a^{2}\right)^{1 / 2}}\right)
\end{gathered}
$$

A máxima deformação ocorre no pólo onde $r=r^{\prime}=0$. Da equação 4.25 obtem-se:

$$
\varepsilon_{\theta_{\max }}=\varepsilon_{\phi_{\max }}=\ln \left(\frac{2 \rho}{\rho+\left(\rho^{2}-a^{2}\right)^{1 / 2}}\right)
$$

Sendo,

$$
h=\rho-\left(\rho^{2}-a^{2}\right)^{1 / 2}
$$

E a equação 4.25 pode ser re-escrita:

$$
\varepsilon_{\theta_{\max }}=-\ln [1-(h / 2 \rho)]=\ln \left[1+(h / a)^{2}\right]
$$

onde

$$
\rho=\left(a^{2}+h^{2}\right) / 2 h
$$




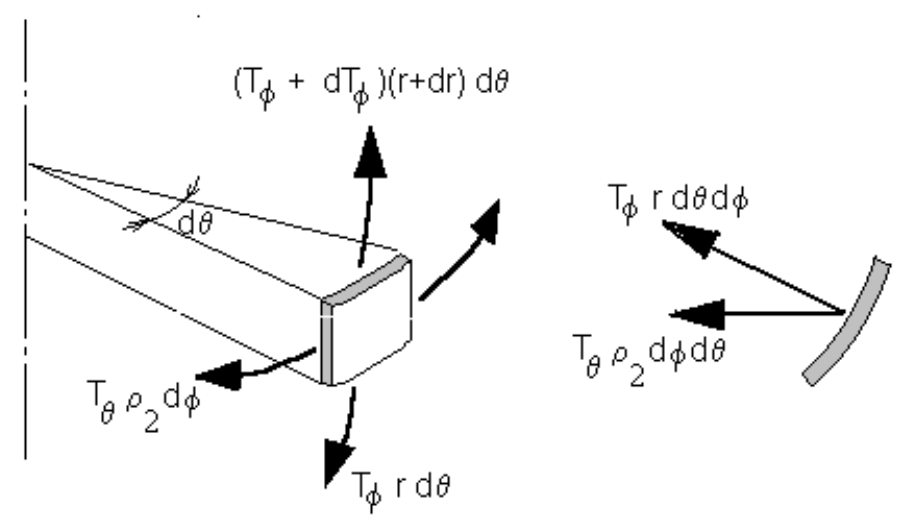

a) Forças atuando num elemento da casca com resultantes radial e normal.

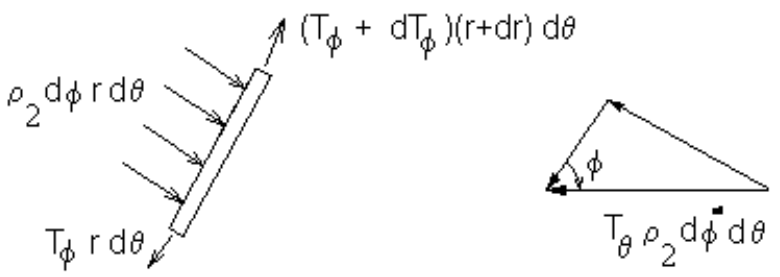

b) Componentes tangencial e normal das forças em um elemento de casca.

Figura 22: Forças atuantes em um elemento de casca (Marciniak e Duncan (1992)).

\subsubsection{Condições de Equilíbrio}

Para uma casca sujeita a um carregamento hidrostático, onde o momento fletor pode ser negligenciado, as forças atuantes em um elemento de casca podem ser representadas na forma da figura 22 a.

As tensões em arco, $T_{\theta}$, dão origem a uma componente radial:

$$
T_{\theta} \rho_{2} \mathrm{~d} \phi \mathrm{d} \theta
$$

Resolvendo estas forças segundo a superfície normal, como representado na figura 22 b,

$$
p \rho_{2} \mathrm{~d} \phi r \mathrm{~d} \theta-T_{\theta} \rho_{2} \mathrm{~d} \phi \mathrm{d} \theta \operatorname{sen} \phi-T_{\phi} r \mathrm{~d} \theta \mathrm{d} \phi=0
$$

e a pressão normal $p$ é:

$$
p=T_{\theta}[\operatorname{sen}(\phi / r)]+T_{\phi} / \rho_{2}
$$


$\mathrm{ou}$

$$
p=T_{\theta} / \rho_{1}+T_{\phi} / \rho_{2}
$$

Resolvendo estas forças tangencialmente, como indicado na figura 22 b,

$$
\left(T_{\phi}+\mathrm{d} T_{\phi}\right)(r+\mathrm{d} r) \mathrm{d} \theta-T_{\phi} r \mathrm{~d} \theta-T_{\theta} \rho_{2} \mathrm{~d} \phi \mathrm{d} \theta \cos \phi=0
$$

Considerando que $\mathrm{d} r / \cos \phi=\rho_{2} \mathrm{~d} \phi$,

$$
\mathrm{d} T_{\phi} / \mathrm{d} r-\left(T_{\theta}-T_{\phi}\right) / r=0
$$

Além disso, as tensões uniformemente distribuídas tangentes à casca, são equivalentes a uma resultante axial $Z$ definida por:

$$
Z=\left(T_{\phi}\right)_{0} 2 \pi r_{0} \operatorname{sen} \phi_{0}
$$

$\mathrm{Ou}$

$$
\left(T_{\phi}\right)_{0}=Z /\left(2 \pi r_{0} \operatorname{sen} \phi_{0}\right)
$$

\subsubsection{Estiramento com Punção Hemisférico Rígido}

O estiramento por meio de um punção difere de várias maneiras do estiramento por pressão hidrostática. Pontos em comum aos dois processos: o estado de tensões é o mesmo em qualquer lugar da chapa; as forças unitárias $T_{\theta}$ e $T_{\phi}$ são iguais:

$$
T_{\theta}=T_{\phi}=T
$$

O perfil da conformação nos dois processos, no entanto é diferente. Enquanto o perfil da conformação hidrostática é aproximadamente esférico, no caso do punção, isso só acontece com as regiões da chapa que entram em contato com o punção. A tensão do contato do punção será constante e, da equação 4.33: 


$$
q=2 T / \rho_{\rho}
$$

Claramente, pode ser percebido que a pressão de contato no caso do estiramento com punção é maior do que no caso do estiramento por pressão hidrostática, pois $\rho_{t}>\rho_{\rho}$.

No ponto de tangência B, o ângulo esférico subentendido é $\phi_{b}$ e a força axial exercida pelo punção na chapa é, da equação 4.36 :

$$
F_{p}=Z=T 2 \pi r_{b} \operatorname{sen} \phi_{b}=T 2 \pi \rho_{\rho} \operatorname{sen}^{2} \phi_{b}
$$

Na região A-B, a pressão atuante na chapa é zero e ambas as tensões principais são iguais. Segue que da equação 4.33:

$$
\rho_{1}=-\rho_{2}
$$

Como a força axial na região livre permanece constante, da equação 4.39:

$$
r^{\prime} \operatorname{sen} \phi=r_{b} \operatorname{sen} \phi_{b}=Z /(2 \pi T)
$$

para $r_{b}<r^{\prime}<a$. E isto define o perfil da região livre.

\subsubsection{Efeito do Atrito}

Se não existir atrito na interface punção-chapa, a distribuição de deformação para uma dado avanço do punção, $h$, pode remontar ao caso do estiramento hidrostático. Isto implica que, na medida em que o punção avança, o material no centro da chapa continua a 'deslizar' sobre a face do punção em direção às bordas do disco. Na presença de atrito, como mostrado na figura 23 a, a deformação próxima ao centro da chapa é reduzida e a maior redução de espessura ocorre próximo ao ponto de tangência B. Pela figura 23 b nota-se que, com atrito, a região que tende a superar primeiro a deformação limite é a 

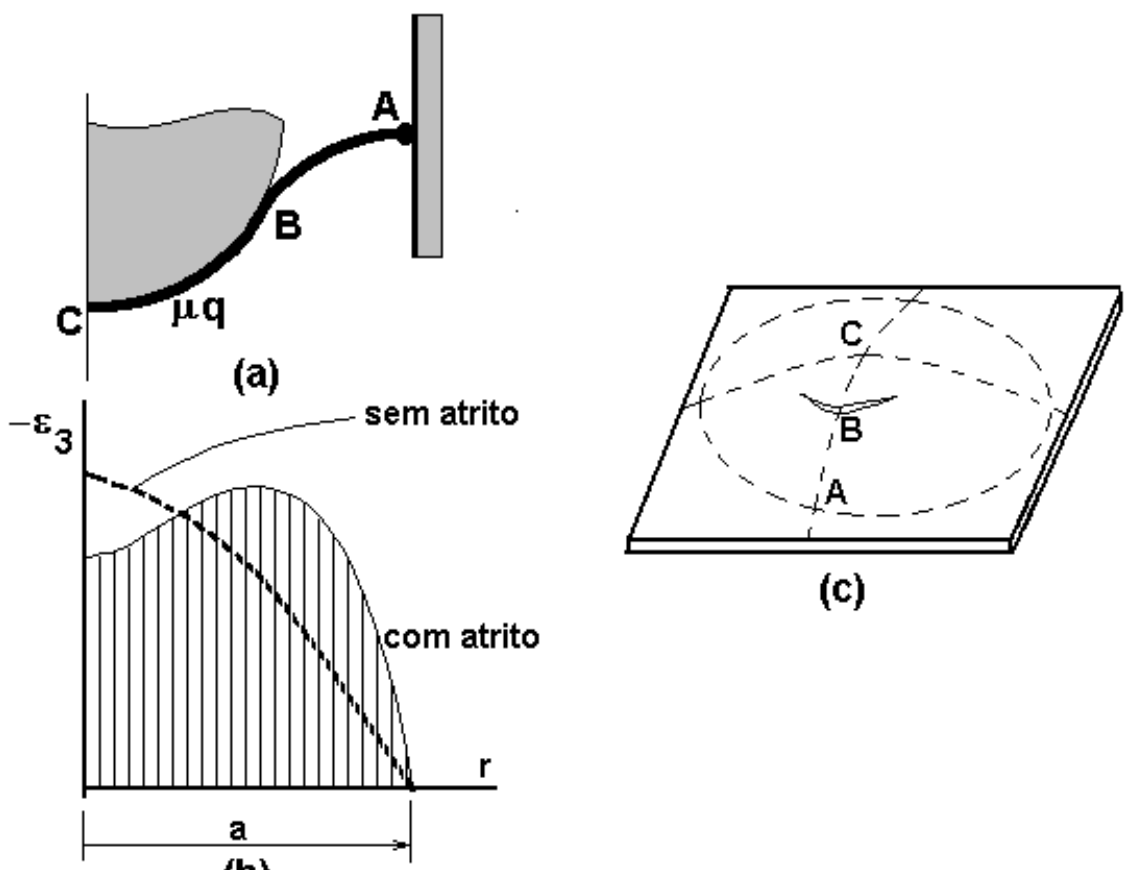

(c)

(b)

Figura 23: (a)Força de atrito na interface punção-chapa; (b) influencia do atrito na deformação; (c) ruptura usualmente próxima a região B (Marciniak e Duncan (1992)).

região próxima a $\mathrm{B}$ e não o pólo, figura 23 c. A proximidade da região limite em relação ao pólo, portanto, depende da eficiência da lubrificação.

\subsection{Estampagem Profunda}

A figura 19 mostra uma típica operação de estampagem, onde pode-se distinguir duas regiões de deformação. Na parte central a chapa é estirada para fora sobre a face do punção. Na região externa a chapa é puxada para dentro da matriz. Na estampagem profunda a chapa não está fixa na matriz, então ela é puxada completamente para para formar um copo, como mostrado na figura 24. Na figura 25 a linha $A$ indica pontos no blank onde os raios permanecem constantes. Abaixo de $A$, o blank é estirado sobre o punção enquanto acima de $A$ o material é empurrado sobre a matriz. A tração em $A$ mostra que há um equilíbrio entre a força que a aba resiste à estampagem e a força aplicada pelo estiramento,figura $25 \mathrm{~b}$. No processo, um blank circular de raio $r_{0}$ e espessura $t_{0}$ é segurado entre uma matriz e um prensa-chapas, e é empurrado por um copo de raio $R$ 


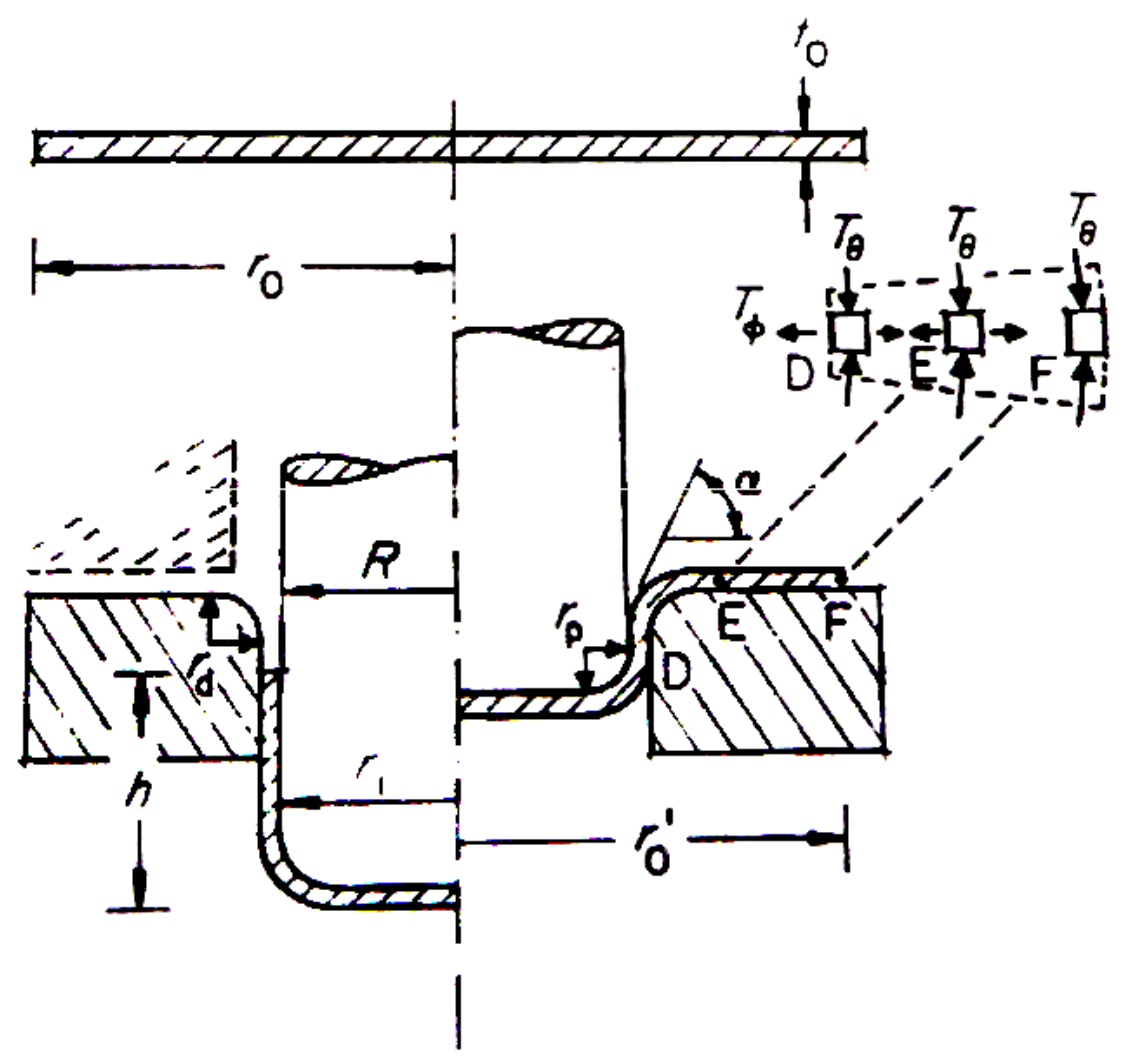

Figura 24: Geometria e forças na estampagem profunda de um copo cilíndrico (Marciniak e Duncan (1992)).

por uma profundidade $h$. O raio da parede do copo é $r_{i}$, o punção tem um raio $r_{p}$ e a matriz tem raio $r_{d}$.

\subsubsection{Deformação da Espessura na Aba}

As forças na aba são mostradas na figura 26 a. Assumindo que a tração efetiva, $\sigma_{f} t=\mathbf{T}$, permanece constante, o estado de tensão pode ser ilustrado em um espaço de tensões oblíquos (figura 26 b). Os vetores deformação são mostrados na figura 26 c e sua projeção no eixo da deformação na espessura dá $\varepsilon_{t}=\ln \left(t / t_{0}\right)$. No contorno da aba o estado de tensão é de compressão uniaxial, com $T_{\phi}=0$ e $T_{\theta}=-\mathbf{T}$. A deformação é

$$
\varepsilon_{\theta}=\ln \frac{r_{0}^{\prime}}{r_{0}}, \quad \varepsilon_{\phi}=\varepsilon_{t}=-\frac{1}{2} \varepsilon_{\theta}
$$



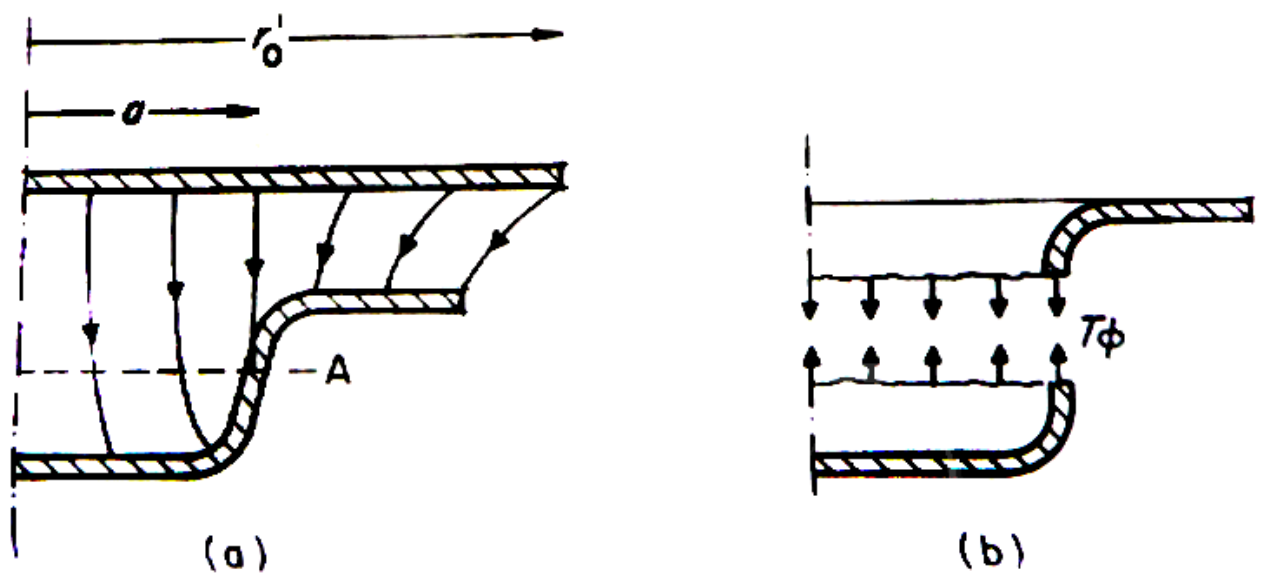

(b)

Figura 25: (a) Regiões de estiramento e estampagem; (b) força máxima Marciniak e Duncan (1992)

onde $r_{0}^{\prime}$ é o raio do blank durante a conformação. A espessura, portanto, irá aumentar,

$$
\varepsilon_{t}=-\frac{1}{2} \ln \frac{r_{0}^{\prime}}{r_{0}}
$$

$\mathrm{Ou}$

$$
t=t_{0}\left(\frac{r_{0}}{r_{0}^{\prime}}\right)^{1 / 2}
$$

No raio interno $r_{i}$, a figura 26 c mostra que a deformação na espessura é negativa, e então, a espessura diminuirá.

\subsubsection{Espessura da Parede}

A aba é empurrada sobre o raio da matriz, sendo dobrada e desdobrada pela ação da tração $T_{\phi}=\ln \left(r_{0}^{\prime} / r_{i}\right)$, causando afinamento. Em uma estampagem completa do copo (figura 27), a espessura na borda do copo deve ser, pela equação 4.45

$$
t_{f}=t_{0}\left(\frac{r_{0}}{r_{i}}\right)^{1 / 2}
$$

Mais abaixo em $E$ onde $T_{\theta} \approx-T_{\phi}$, a espessura não muda

$$
t_{e} \cong t_{0}
$$




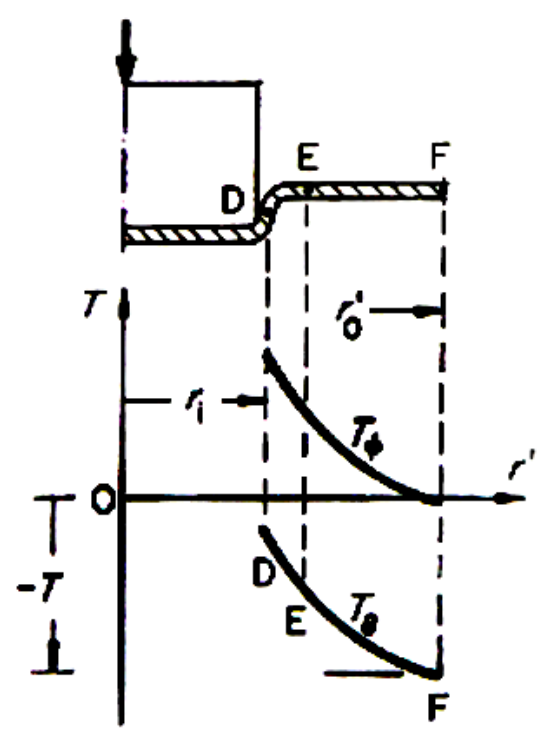

(a)

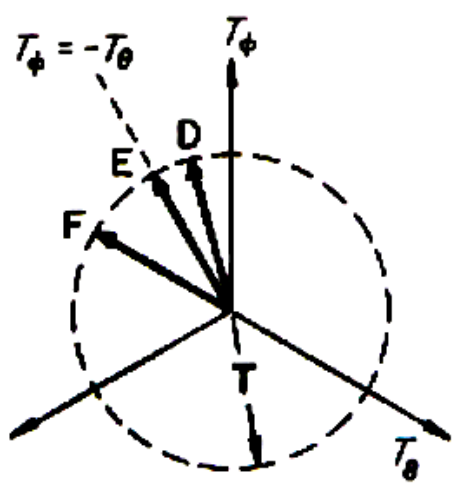

(b)

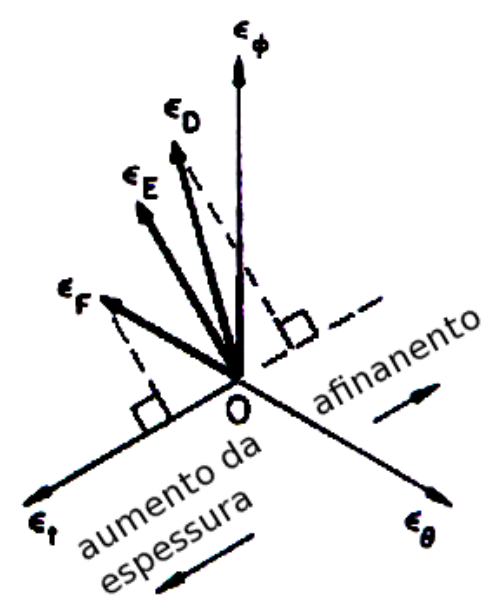

(c)

Figura 26: (a) Forças na aba; (b) coordenadas em um espaço oblíquo; (c) deformações no espaço oblíquo (Marciniak e Duncan (1992)).

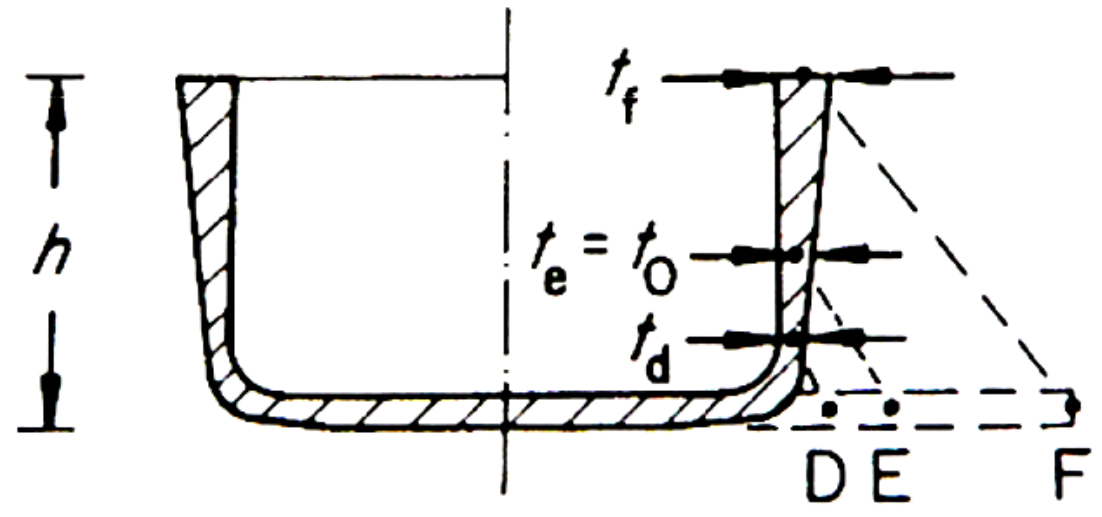

Figura 27: Espessura na parede do copo (Marciniak e Duncan (1992)).

enquanto perto da base

$$
t_{d}<t_{0}
$$

Em $D$, a região está sujeita a dobramento e desdobramento no raio da matriz, e dobramento no raio do punção que, para grande razões de estampagem, $r_{0} / R$, afinamento local pode ser observado. 


\subsubsection{Efeito do Atrito do Prensa-chapas}

O prensa-chapas é usado para prensar a chapa contra a matriz para previnir o aparecimento de orelhas, durante o processo de estampagem. Geralmente, uma força $B$ constante é aplicada no prensa-chapas, e como o afinamento ocorre no contorno da aba, a força de atrito radial provocada por $B$ atua como na figura 28 .

A força de atrito em cada lado do blank é $\mu B$, onde $\mu$ é o coeficiente de atrito de Coulomb e é distribuido ao longo da periferia de comprimento $2 \pi r_{0}^{\prime}$. Isto é equivalente a uma tração no contorno de

$$
T_{\phi_{0}}=\frac{2 \mu B}{2 \pi r_{0}^{\prime}}=\frac{\mu B}{\pi r_{0}^{\prime}}
$$

na estampagem tem-se $T_{\phi}-T_{\theta}=\mathbf{T}$ que é válido para $0<T_{\phi}<\mathbf{T}$ e $T_{\theta}<0$, substituindo em 4.35 tem-se

$$
\frac{\mathrm{d} T_{\phi}}{\mathrm{d} r}+\frac{\mathbf{T}}{r}=0
$$

integrando fica

$$
T_{\phi}=-\mathbf{T} \ln r+c
$$

e para a condição de contorno $T_{\phi}=T_{\phi_{0}}$ em $r=r_{0}^{\prime}$, obtém-se

$$
T_{\phi}=\mathbf{T} \ln \frac{r_{0}^{\prime}}{r}+\frac{\mu B}{\pi r_{0}^{\prime}}
$$

\subsubsection{Atrito no Raio da Matriz}

Se uma tira é puxada sobre um cilindro, como na figura 29, a pressão de contato é $p=T_{\phi} / \rho$, onde $\rho=r_{d}+(t / 2)$. A mudança na tração devido ao atrito, $\Delta T_{\phi}$, sobre um arco de comprimento $\rho \mathrm{d} \phi$ é

$$
\Delta T_{\phi}=\mu p \rho \mathrm{d} \phi=\mu T_{\phi} \mathrm{d} \phi
$$

integrando a equação acima

$$
\int_{T_{\phi_{1}}}^{T_{\phi_{2}}} \frac{\mathrm{d} T_{\phi}}{T_{\phi}}=\int_{0}^{\phi} \mu \mathrm{d} \phi
$$




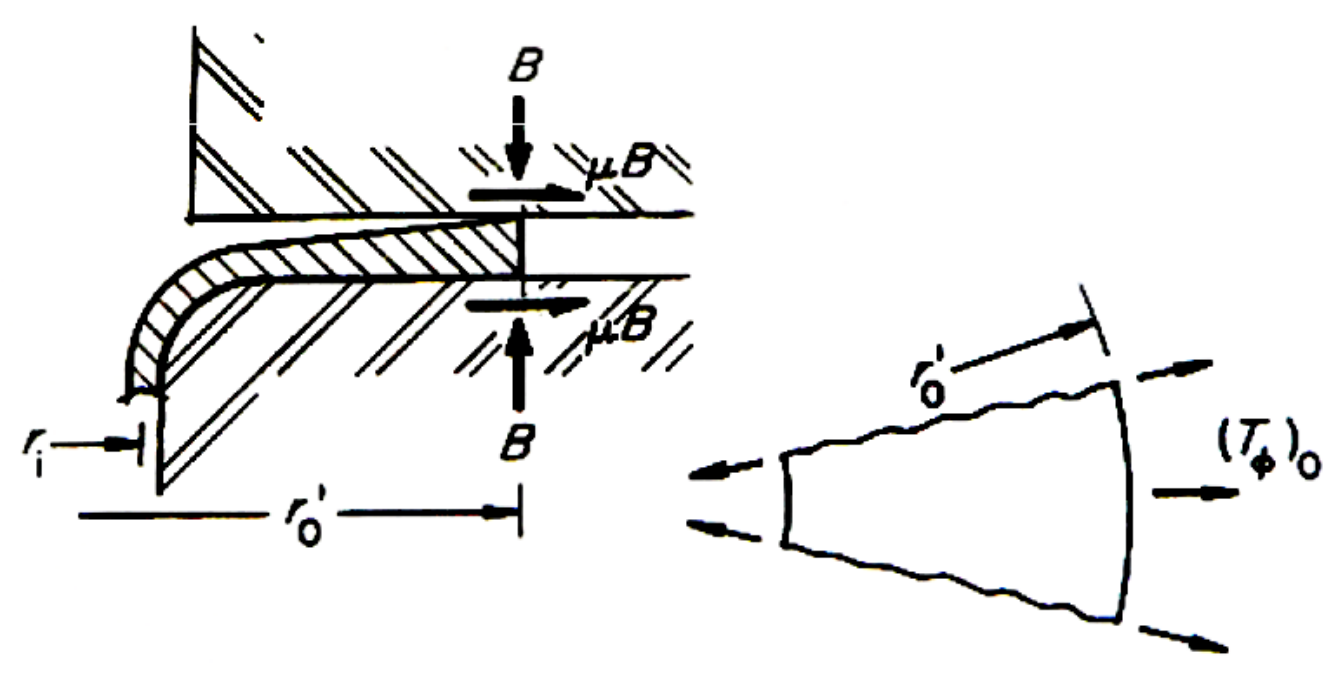

Figura 28: Forças de atrito no prensa-chapas (Marciniak e Duncan (1992)).

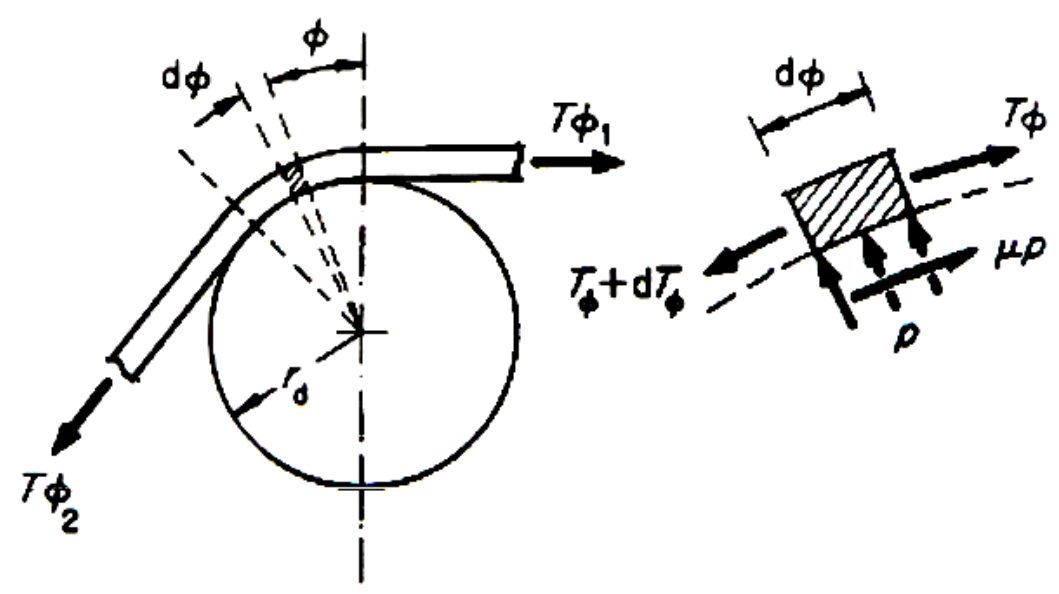

Figura 29: Efeito do atrito em uma tira puxada em um cilindro (Marciniak e Duncan (1992)).

ou seja

$$
\frac{T_{\phi_{2}}}{T_{\phi_{1}}}=\exp (\mu \phi)
$$

A condição no raio do punção, figura 30, é próxima do que ocorre na figura 29 e a tração aumentada pelo atrito é

$$
T_{\phi_{1}}=T_{\phi} \exp (\mu \alpha)
$$




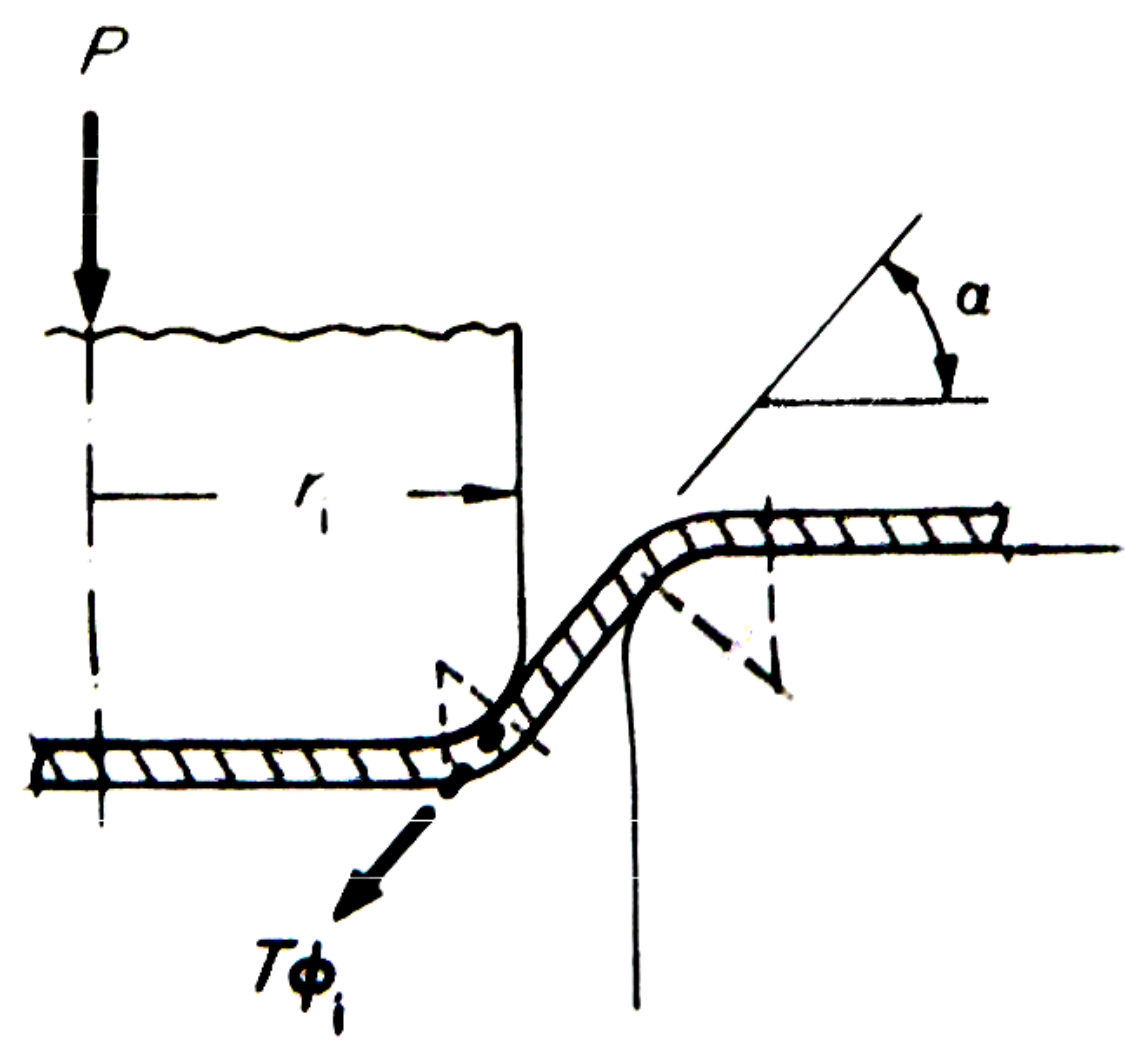

Figura 30: Força no punção (Marciniak e Duncan (1992)).

\subsubsection{Efeito do Contato de Pressão no Punção Cilíndrico}

Assumindo que há uma tração biaxial na região de estiramento, isto é,

$$
T_{\theta}=T_{\phi}=\mathbf{T}
$$

se a tração é suficiente para causar deformação plastica e estiramento em toda a área. Se a razão de dobramento, $r_{p} / t$, no raio do punção for pequena, como na figura 31 , a pressão de contato pode ser suficiente para influenciar no escoamento, e o estado plano de tensão não será mais válido. Da equação 4.33 onde $\rho_{1}=r_{p}$ a $\rho_{2} \approx r_{i}$, a tensão de contato em $B$ é

$$
p_{b}=\mathbf{T}\left(\frac{1}{r_{i}}+\frac{1}{r_{p}}\right)
$$

Se a pressão de contato fosse desconsiderada, e assumindo que a chapa deforme no 


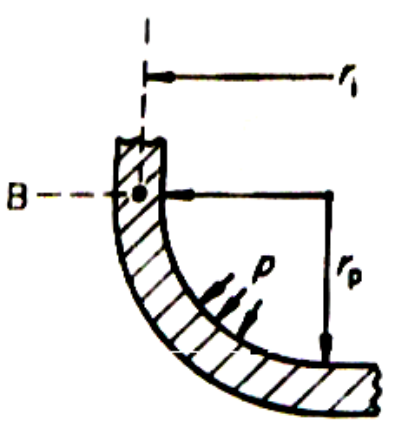

(a)

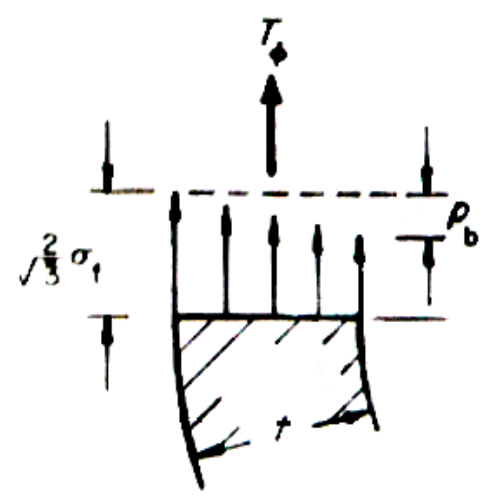

(b)

Figura 31: (a)Pressão de contato no raio do punção; (b) distribuição da tensão (Marciniak e Duncan (1992)).

estado plano de deformação, $\mathrm{d} \varepsilon_{\theta}=0$, então a força $T_{\phi}$ poderia ser escrita

$$
T_{\phi}=\frac{2}{\sqrt{3}} \sigma_{f} t
$$

Se a tração média através da tensão na espessura, $\sigma_{3}$, é $p_{b} / 2$, figura 31 b, a força pode ser reduzida para

$$
T_{\phi}=\left(\frac{2}{\sqrt{3}} \sigma_{f}-\frac{p_{b}}{2}\right) t
$$

Combinando as equações 4.58 e 4.59 obtém-se

$$
T_{\phi} \approx \frac{2}{\sqrt{3}} \sigma_{f} t\left(1-\frac{1}{2}\left(\frac{t}{r_{i}}+\frac{t}{r_{p}}\right)\right)
$$

\subsubsection{Afinamento no Raio do Punção}

O afinamento na chapa, no raio do punção, ocorre pela tração de dobramento. Assumindo que linha neutra ocorre como na figura 32 , temos

$$
t_{0} r_{p} r_{i} \approx t\left(r_{p}+(t / 2)\right)\left(r_{i}+(t / 2)\right)
$$




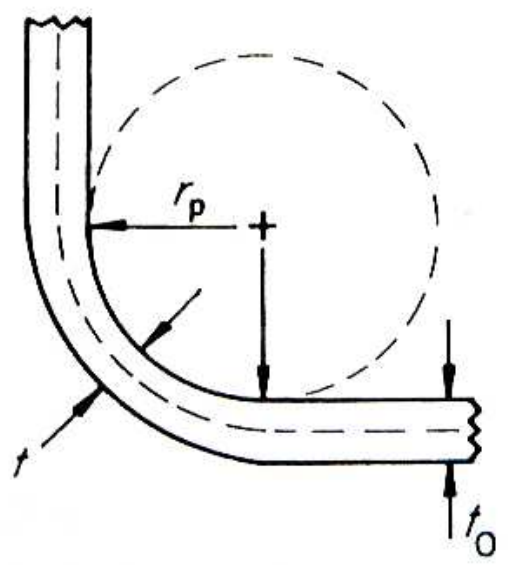

Figura 32: Afinamento no raio do punção (Marciniak e Duncan (1992)).

Da equação acima

$$
t \approx \frac{t_{0}}{1+\frac{1}{2}\left(\frac{t}{r_{i}}+\frac{t}{r_{p}}\right)}
$$

Combinando a equação acima com a equação 4.61 e escrevendo $\mathbf{T}=\sigma t_{0}$, obtém-se

$$
\begin{aligned}
& T_{\phi}=\frac{2}{\sqrt{3}} \sigma_{f} t_{0} \frac{1-\frac{1}{2}\left(\frac{t}{r_{i}}+\frac{t}{r_{p}}\right)}{1+\frac{1}{2}\left(\frac{t}{r_{i}}+\frac{t}{r_{p}}\right)} \\
& \approx \frac{2}{\sqrt{3}} \sigma_{f} t_{0}\left(1-\frac{t}{r_{i}}-\frac{t}{r_{p}}\right)
\end{aligned}
$$

que indica a redução na resistência da parede do copo neste ponto, devido aos efeitos ambos da pressão de contato e afinamento.

\subsubsection{Atrito no Punção}

Se a parede do copo está escoando, então, no lado do punção para a distância $h_{w}$, figura 33, a parede está deformando no estado plano de deformação onde

$$
\mathrm{d} \varepsilon_{\theta}=\beta \mathrm{d} \varepsilon_{\phi}=0
$$




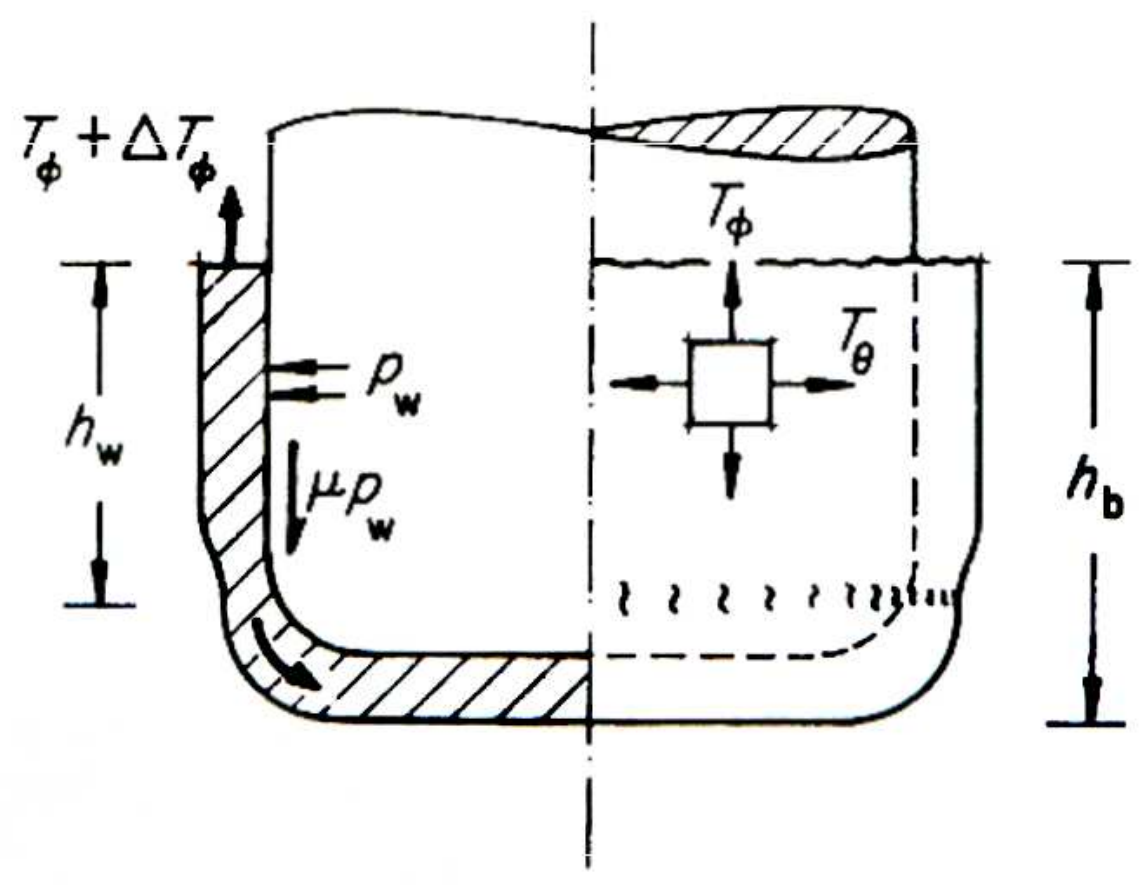

Figura 33: Atrito na parede do punção (Marciniak e Duncan (1992)).

assim temos

$$
T_{\theta}=\frac{T_{\phi}}{2}
$$

A pressão de contato na parede do punção, da equação 4.33, é

$$
p_{w}=\frac{T_{\theta}}{\rho_{1}} \approx \frac{1}{2} \frac{T_{\phi}}{r_{i}}
$$

onde $\rho_{1} \approx r_{i}$ e $\rho_{2}=\infty$.

Se a interface de atrito é $\mu p_{w}$, o equação de equilíbrio para uma unidade de largura circunferencial da parte cilíndrica do copo é

$$
T_{\phi}+\Delta T_{\phi}-h_{w} \mu p_{w}-T_{\phi}=0
$$

ou seja

$$
\Delta T_{\phi}=\frac{\mu h_{w}}{2 r_{i}} T_{\phi}
$$

A parte central do copo é progressivamente envolvida e estirada ao redor do punção, figura 34 a. A força no fundo do copo, $T_{\phi}$, irá aumentar daquela requirida para iniciar o escoamento plástico, $T_{y b}$, para o máximo, $T_{b \max }$, quando a falha por afinamento lo- 

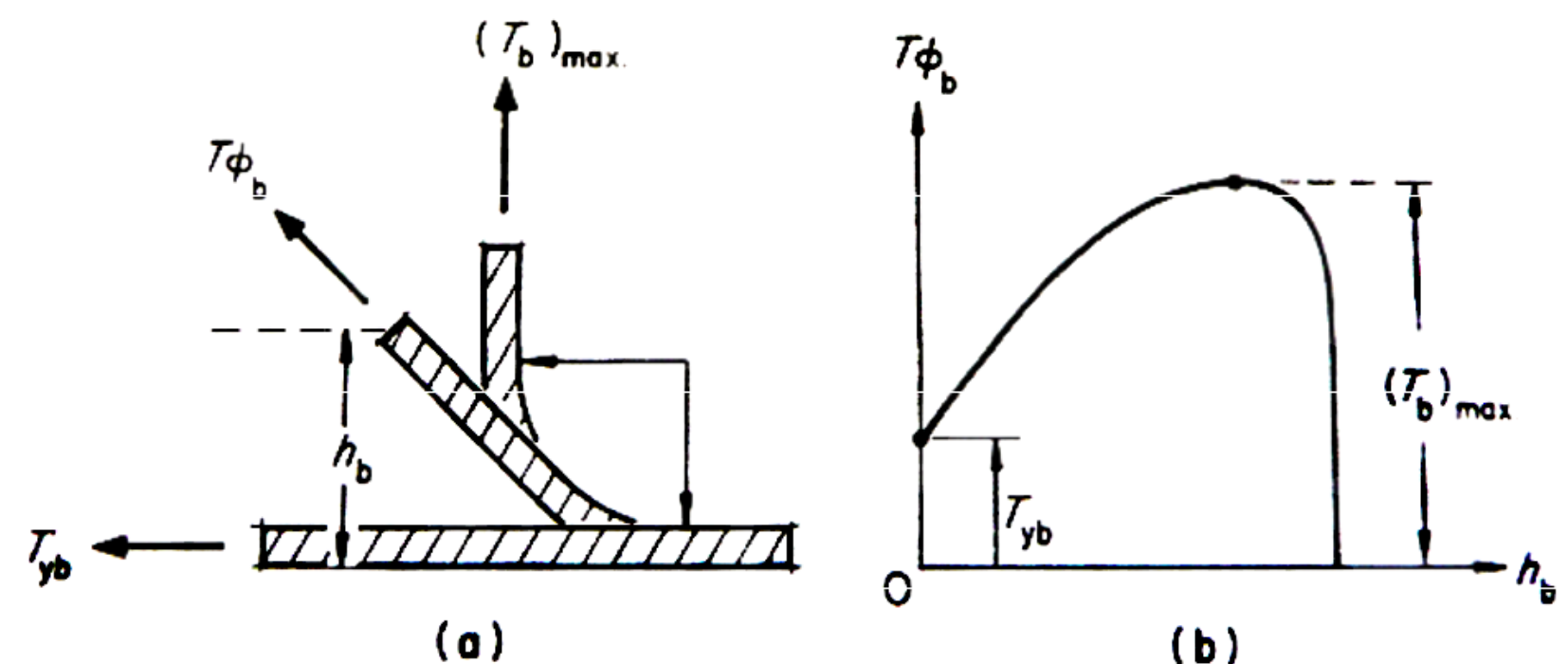

Figura 34: (a) Variação na força $T_{\phi b}$, de estiramento no fundo, com a profundidade $h_{b}$ da região estirada (Marciniak e Duncan (1992)).

cal ocorrerá, figura 34 b. Este afinamento geralmente ocorre acima do raio do punção, figura 33 . 


\section{Modelagem do Processo de Estampagem pelo Método dos Elementos Finitos}

A abordagem variacional é aqui usada para a derivação das equações básicas de elementos finitos. Ela requer que entre os deslocamentos admissíveis $u_{i}$ satisfaçam as condições de compatibilidade, bem como as condições de contorno.

\subsection{Abordagem Variacional}

A solução pela abordagem variacional dá ao funcional, um valor estacionário:

$$
\pi=\int_{V} \bar{\sigma} \bar{\varepsilon} d V-\int_{S} F_{i} u_{i} d S \quad \text { para materiais rígido-plásticos }
$$

onde $\bar{\sigma}$ é a tensão efetiva, $\bar{\varepsilon}$ é a deformação efetiva, $F_{i}$ representa as forças de tração de superfície, $V$ o volume e $S$ a superfície.

De acordo com a abordagem variacional, a variação de primeira ordem do funcional é zero:

$$
\delta \pi=\int_{V} \bar{\sigma} \delta \bar{\varepsilon} d V-\int_{S} F_{i} \delta u_{i} d S=0
$$

onde para materiais rígido-plásticos $\bar{\sigma}=\bar{\sigma}(\bar{\varepsilon})$.

A equação 5.2 é a equação básica para elementos finitos para o caso de deformações no plano definido em um estado plano de tensões. 


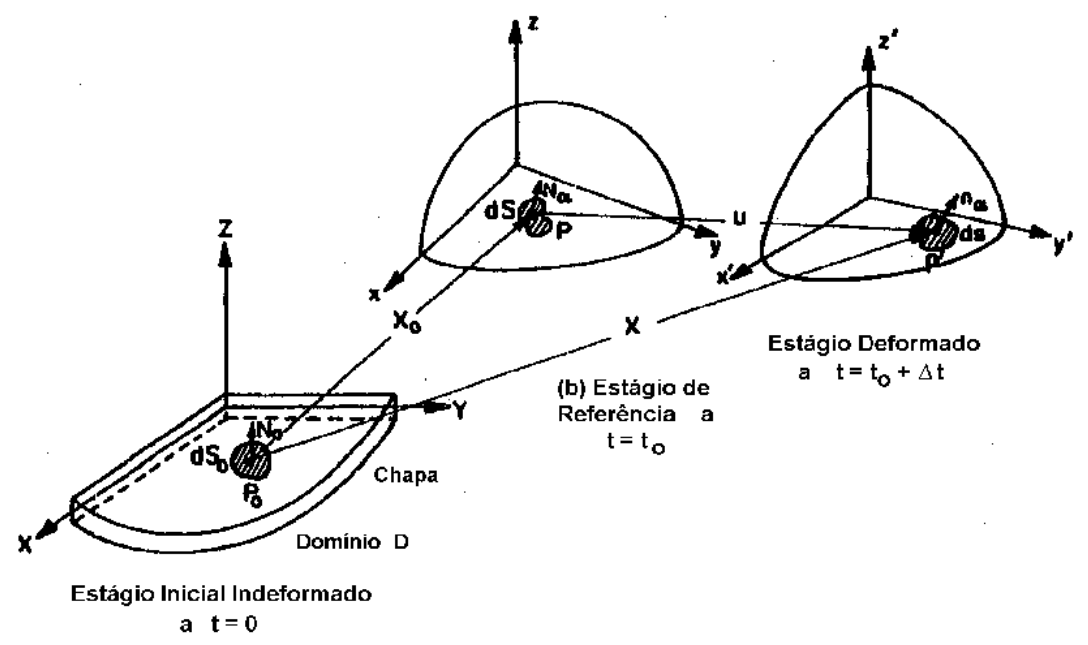

Figura 35: Cinemática da deformação do elemento de chapa nos vários estágios (Toh (1983)).

\subsection{Cinemática da Deformação}

Um estado de tensões é dito plano quando uma chapa é carregada ao longo de suas extremidades, segundo o plano da chapa (a direção normal a esse plano é uma direção de tensão principal cuja magnitude é zero). Deformação no plano da chapa, como a conformação de uma flange por estampagem é um problema de estado plano de tensão. Deformações fora do plano de chapas metálicas como dobramento e estampagem profunda de produtos em forma de copo, por exemplo, requerem solução pelo uso da Teoria da Membrana. Esta teoria despreza variações de tensão na direção da espessura da chapa e considera a distribuição das componentes de tensão somente no plano da chapa. Deste modo, as funções para análise de deformações no plano e fora do plano consideram somente componentes de tensão atuantes no plano da chapa. No entanto, as análises de deformações fora do plano requerem a consideração de grandes deformações, enquanto que a teoria infinitesimal é aplicável para análises de deformações no plano (Kobayashi et al. (1989)).

Na figura 35, tem-se uma análise da cinemática da deformação em um domínio cujo corpo é uma chapa. As expressões que definem o vetor força $d \mathbf{F}$, para os estágios de 
referência e deformado são:

$$
\begin{aligned}
& d \mathbf{F}=P_{\alpha \beta} N_{\alpha} \mathbf{g}_{\beta} d S_{0} \\
& d \mathbf{F}=\sigma_{\alpha \beta} n_{\alpha} \mathbf{g}_{\beta} d S
\end{aligned}
$$

onde $n_{\alpha}$ e $N_{\alpha}$ representam o vetor normal à superfície $d S$ e $d s$ do elemento, em ambas as configurações, $\mathbf{g}_{\beta}$ é o vetor base de sistema de referência $X^{\prime}-Y^{\prime}-Z^{\prime}, P_{\alpha \beta}$ são os componentes do primeiro tipo, do tensor tensão de Piola-Kirchhoff e $\sigma_{\alpha \beta}$ os componentes do tensor tensão de Cauchy. Os sistemas de coordenadas $X-Y-Z$ podem ser curvos e não são necessariamente os mesmos.

A equação (5.3) ainda pode ser escrita, na configuração de referência, como

$$
d \mathbf{F}=\left[u_{\beta}+\delta_{\beta \gamma}\right] S_{\alpha \gamma} N_{\alpha} \mathbf{g}_{\beta} d S
$$

onde $u_{\beta}$ é a derivada do vetor deslocamento $u, \delta_{\beta \gamma}$ é o delta de Kronecker e $S_{\alpha \gamma}$ são os componentes do tensor segundo de Piola-Kirchhoff (PK-2). Relembrando, a relação entre os três tensores pode ser escrita como:

$$
J \sigma_{\alpha \beta}=x_{\alpha, \gamma} P_{\beta \gamma}=x_{\alpha, \gamma} x_{\beta, \delta} S_{\gamma \delta}
$$

sendo $x_{\alpha, \gamma}=\frac{\partial x_{\alpha}}{\partial x_{\gamma}}=F, x_{\beta, \delta}=\frac{\partial x_{\beta}}{\partial x_{\delta}}=F$ e $J=\operatorname{det}(F)$, onde $F=$ gradiente de deformação.

Considera-se o estado de equilíbrio na configuração deformada, como no caso de chapas as forças de corpo são desprezíveis, tem-se a equação:

$$
\delta w=\int_{S} \mathbf{f} \cdot \delta \mathbf{u} d S
$$

onde $\delta w$ é o trabalho virtual total feito pelas forças de tração externas sobre a superfície $S, \mathbf{f}$ é a força de tração de superfície expressa por unidade de área da configuração de referência, $\mathbf{f}=\frac{d \mathbf{F}}{d S}$ e $\delta \mathbf{u}$ é o vetor de deslocamento virtual. 
Da equação (5.3) e da relação $\delta \mathbf{u}=\delta u_{\gamma} \mathbf{g}_{\gamma}$, a expressão (5.7) pode ser escrita

$$
\delta w=\int_{S_{0}}\left[P_{\alpha \beta} N_{\alpha} \mathbf{g}_{\beta}\right]\left[\delta u_{\gamma} \mathbf{g}_{\gamma}\right] d S_{0}=\int_{S_{0}} P_{\alpha \beta} N_{\alpha} \delta u_{\beta} d S_{0}
$$

desde que $\mathbf{g}_{\beta} \cdot \mathbf{g}_{\gamma}=\delta_{\beta \gamma}$ é o delta de Kronecker, e pelo teorema do divergente,

$$
\delta w=\int_{V}\left[P_{\alpha \beta} \delta u_{\beta}\right]_{, \alpha} d V=\int_{V}\left[P_{\alpha \beta} \delta u_{\beta, \alpha}+P_{\alpha \beta, \alpha} \delta u_{\beta}\right] d V
$$

onde $d V$ é o volume do elemento na configuração $t=t o$. Considerando no estado de equilíbrio, $P_{\alpha \beta, \alpha}=0$ então:

$$
\delta w=\int_{V} P_{\alpha \beta} \delta u_{\beta, \alpha} d V=\int_{S_{0}} \mathbf{f} \cdot \delta \mathbf{u} d S_{0}
$$

da eq.(5.6) temos:

$$
P_{\alpha \beta}=\left[u_{\beta, \gamma}+\delta_{\beta \gamma}\right] S_{\alpha \beta}
$$

e a eq.(5.10) pode ser escrita como

$$
\delta w=\int_{V}\left[u_{\beta, \gamma}+\delta_{\beta \gamma}\right] S_{\alpha \beta} \delta u_{\beta, \alpha} d V=\frac{1}{2} \int S_{\alpha \gamma} \delta\left[u_{\gamma, \alpha}+u_{\alpha, \gamma}+u_{\beta, \gamma} u_{\beta, \alpha}+\delta_{\alpha \gamma}\right]
$$

Introduzindo o tensor de deformação de Green

$$
d E_{\alpha \beta}=\frac{1}{2}\left[u_{\alpha, \beta}+u_{\beta, \alpha}+u_{\gamma, \alpha} u_{\gamma, \beta}\right]
$$

Do princípio do trabalho virtual temos o seguinte funcional:

$$
\delta w=\int_{S} \mathbf{f} \cdot \delta \mathbf{u} d S=\int_{V} S_{\alpha \beta} \delta\left(d E_{\alpha \beta}\right) d V
$$

Consequentemente, a forma variacional do funcional pode ser obtida de (5.14) e o problema de valor estacionário pode ser formulado pela condição de nulidade da variação da funcional:

$$
\delta \phi=\int_{V} S_{\alpha \beta} \delta\left(d E_{\alpha \beta}\right) d V-\int_{S} \mathbf{f} \cdot \delta \mathbf{u} d S=0
$$

A expressão do trabalho incremental fica

$$
d w=S_{\alpha \beta} d E_{\alpha \beta}=\bar{S} d \bar{E}
$$




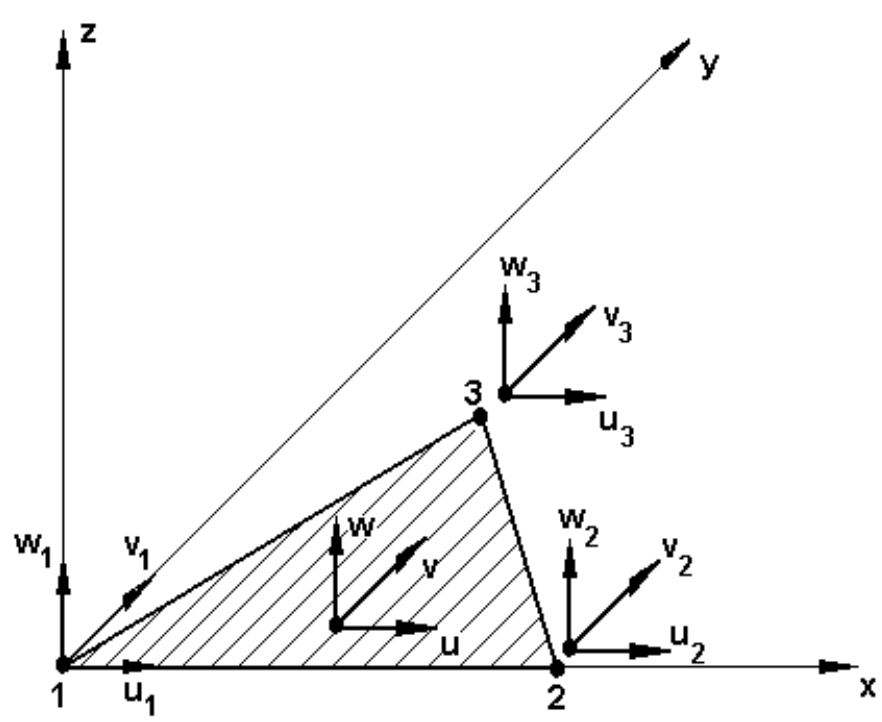

Figura 36: Coordenadas locais do elemento triangular com os deslocamentos nodais $u, v$ e w (Toh (1983)).

onde $\bar{S}$ e $d \bar{E}$ são a tensão e deformação incremental efetiva. A expressão (5.15) pode ser expressa na forma:

$$
\delta \phi=\int_{V} \bar{S} \delta(d \bar{E}) d V-\int_{S} \mathbf{f} \cdot \delta \mathbf{u} d S=0
$$

\subsection{Discretização}

A equação (5.17) é a equação a ser usada na discretização para o método de elementos finitos para o caso de materiais rígido-plásticos. Para a discretização é usada a configuração de elementos de forma triangular vantajosa quando são analisadas estruturas planas. É assumido que durante a deformação os elementos permanecem planos e assim, o efeito de dobramento pode ser negligenciado.

De modo a agilizar o procedimento, o cálculo das matrizes de elementos é feito em um sistema de coordenadas locais, e depois, a transformação das matrizes para o sistema global, que é o sistema comum para todos os elementos.

Dividindo a chapa metálica em elementos triangulares, e considerando um sistema de coordenadas cartesianas local para um elemento, o campo de deslocamentos, para um elemento tipo membrana triangular, dado por u, v e w (figura 36) as funções lineares de 
deslocamento no elemento serão:

$$
\begin{aligned}
& u=a_{1}+b_{1} x+c_{1} y \\
& v=a_{2}+b_{2} x+c_{2} y \\
& w=a_{3}+b_{3} x+c_{3} y
\end{aligned}
$$

$\mathrm{ou}$

$$
\left\{\begin{array}{l}
u \\
v \\
w
\end{array}\right\}=\left[\begin{array}{lllllllll}
1 & x & y & 0 & 0 & 0 & 0 & 0 & 0 \\
0 & 0 & 0 & 1 & x & y & 0 & 0 & 0 \\
0 & 0 & 0 & 0 & 0 & 0 & 1 & x & y
\end{array}\right]\left\{\begin{array}{c}
a_{1} \\
b_{1} \\
c_{1} \\
a_{2} \\
b_{2} \\
c_{2} \\
a_{3} \\
b_{3} \\
c_{3}
\end{array}\right\}
$$

e a equação 5.20, para os pontos nodais fica:

$$
\{\mathbf{u}\}=\mathbf{A}\{\varphi\}
$$

onde

$$
\{\mathbf{u}\}^{T}=\left[\begin{array}{lllllllll}
u_{1} & v_{1} & w_{1} & u_{2} & v_{2} & w_{2} & u_{3} & v_{3} & w_{3}
\end{array}\right]
$$




$$
A=\left[\begin{array}{ccccccccc}
1 & x_{1} & y_{1} & 0 & 0 & 0 & 0 & 0 & 0 \\
0 & 0 & 0 & 1 & x_{1} & y_{1} & 0 & 0 & 0 \\
0 & 0 & 0 & 0 & 0 & 0 & 1 & x_{1} & y_{1} \\
1 & x_{2} & y_{2} & 0 & 0 & 0 & 0 & 0 & 0 \\
0 & 0 & 0 & 1 & x_{2} & y_{2} & 0 & 0 & 0 \\
0 & 0 & 0 & 0 & 0 & 0 & 1 & x_{2} & y_{2} \\
1 & x_{3} & y_{3} & 0 & 0 & 0 & 0 & 0 & 0 \\
0 & 0 & 0 & 1 & x_{3} & y_{3} & 0 & 0 & 0 \\
0 & 0 & 0 & 0 & 0 & 0 & 1 & x_{3} & y_{3}
\end{array}\right]
$$

e

$$
\{\varphi\}^{t}=\left[\begin{array}{lllllllll}
a_{1} & b_{1} & c_{1} & a_{2} & b_{2} & c_{2} & a_{3} & b_{3} & c_{3}
\end{array}\right]
$$

Combinando as equações (5.20) e (5.21):

$$
\left\{\begin{array}{c}
u \\
v \\
w
\end{array}\right\}=\mathbf{N}\{\mathbf{u}\}
$$

onde $\mathbf{N}$ é a matriz interpolação, na forma

$$
\mathbf{N}=\left[\begin{array}{lllllllll}
1 & x & y & 0 & 0 & 0 & 0 & 0 & 0 \\
0 & 0 & 0 & 1 & x & y & 0 & 0 & 0 \\
0 & 0 & 0 & 0 & 0 & 0 & 1 & x & y
\end{array}\right] \mathbf{A}^{-1}
$$

Em geral, a equação (5.22) pode ser escrita como:

$$
u=\frac{1}{2 \Delta}\left\{\sum_{i=1}^{3}\left(a_{i}+b_{i} x+c_{i} y\right) u_{i}\right\}
$$

onde $a_{1}=x_{2} y_{3}-y_{2} x_{3}, a_{2}=x_{3} y_{1}-y_{3} x_{1}, a_{3}=x_{1} y_{2}-y_{1} x_{2}, b_{1}=y_{2}-y_{3}, b_{2}=y_{3}-y_{1}$, 
$b_{3}=y_{1}-y_{2}, c_{1}=x_{3}-x_{2}, c_{2}=x_{1}-x_{3}, c_{3}=x_{2}-x_{1} \mathrm{e}$

$$
2 \Delta=\left|\begin{array}{ccc}
1 & x_{1} & y_{1} \\
1 & x_{2} & y_{2} \\
1 & x_{3} & y_{3}
\end{array}\right|=2 \quad \mathrm{x} \quad \text { área do elemento triangular }
$$

Os gradientes de deslocamento, de (5.23) são

$$
\begin{array}{ll}
\frac{\partial u}{\partial x}=\frac{1}{2 \Delta}\left(b_{1} u_{1}+b_{2} u_{2}+b_{3} u_{3}\right) ; \quad \frac{\partial u}{\partial y}=\frac{1}{2 \Delta}\left(c_{1} u_{1}+c_{2} u_{2}+c_{3} u_{3}\right) \\
\frac{\partial v}{\partial x}=\frac{1}{2 \Delta}\left(b_{1} v_{1}+b_{2} v_{2}+b_{3} v_{3}\right) ; \quad \frac{\partial v}{\partial y}=\frac{1}{2 \Delta}\left(c_{1} v_{1}+c_{2} v_{2}+c_{3} v_{3}\right) \\
\frac{\partial w}{\partial x}=\frac{1}{2 \Delta}\left(b_{1} w_{1}+b_{2} w_{2}+b_{3} w_{3}\right) ; \quad \frac{\partial w}{\partial y}=\frac{1}{2 \Delta}\left(c_{1} w_{1}+c_{2} w_{2}+c_{3} w_{3}\right)
\end{array}
$$

$$
\left\{\begin{array}{c}
\frac{\partial u}{\partial x} \\
\frac{\partial u}{\partial y} \\
\frac{\partial v}{\partial x} \\
\frac{\partial v}{\partial y} \\
\frac{\partial w}{\partial x} \\
\frac{\partial w}{\partial y}
\end{array}\right\}=\frac{1}{2 \Delta}\left[\begin{array}{ccccccccc}
b_{1} & 0 & 0 & b_{2} & 0 & 0 & b_{3} & 0 & 0 \\
c_{1} & 0 & 0 & c_{2} & 0 & 0 & c_{3} & 0 & 0 \\
0 & b_{1} & 0 & 0 & b_{2} & 0 & 0 & b_{3} & 0 \\
0 & c_{1} & 0 & 0 & c_{2} & 0 & 0 & c_{3} & 0 \\
0 & 0 & b_{1} & 0 & 0 & b_{2} & 0 & 0 & b_{3} \\
0 & 0 & c_{1} & 0 & 0 & c_{2} & 0 & 0 & c_{3}
\end{array}\right]\left\{\begin{array}{c}
u_{1} \\
v_{1} \\
w_{1} \\
u_{2} \\
v_{2} \\
w_{2} \\
u_{3} \\
v_{3} \\
w_{3}
\end{array}\right\}
$$

os componentes de deformação Lagrangeanos se relacionam com os gradientes de deslocamento

$$
\begin{aligned}
d E_{x} & =\frac{\partial u}{\partial x}+\frac{1}{2}\left\{\left(\frac{\partial u}{\partial x}\right)^{2}+\left(\frac{\partial v}{\partial x}\right)^{2}+\left(\frac{\partial w}{\partial x}\right)^{2}\right\} \\
d E_{y} & =\frac{\partial v}{\partial y}+\frac{1}{2}\left\{\left(\frac{\partial u}{\partial y}\right)^{2}+\left(\frac{\partial v}{\partial y}\right)^{2}+\left(\frac{\partial w}{\partial y}\right)^{2}\right\} \\
d E_{x y} & =\frac{1}{2}\left\{\frac{\partial u}{\partial y}+\frac{\partial v}{\partial x}+\frac{\partial u}{\partial x} \frac{\partial u}{\partial y}+\frac{\partial v}{\partial x} \frac{\partial v}{\partial y}+\frac{\partial w}{\partial x} \frac{\partial w}{\partial y}\right\} \\
d E_{z} & =-\left(d E_{x}+d E_{y}\right)
\end{aligned}
$$


$\mathrm{Ou}$

$$
\{d \mathbf{E}\}=\mathbf{B}\{\mathbf{u}\}
$$

onde

$$
\{d \mathbf{E}\}^{T}=\left[\begin{array}{lll}
d E_{x} & d E_{y} & d E_{x y}
\end{array}\right]
$$

A matriz de deformação-deslocamento, B, que é uma função do deslocamento, contém termos lineares e não lineares

$$
\begin{aligned}
\mathbf{B}=\frac{1}{2 \Delta}\left[\begin{array}{lllllllll}
b_{1} & 0 & 0 & b_{2} & 0 & 0 & b_{3} & 0 & 0 \\
0 & c_{1} & 0 & 0 & c_{2} & 0 & 0 & c_{3} & 0 \\
\frac{c_{1}}{2} & \frac{b_{1}}{2} & 0 & \frac{c_{2}}{2} & \frac{b_{2}}{2} & 0 & \frac{c_{3}}{2} & \frac{b_{3}}{2} & 0
\end{array}\right]+ \\
\frac{1}{8 \Delta^{2}}\left[\begin{array}{lllllllllll}
B_{11} & B_{12} & B_{13} & B_{14} & B_{15} & B_{16} & B_{17} & B_{18} & B_{19} \\
B_{21} & B_{22} & B_{23} & B_{24} & B_{25} & B_{26} & B_{27} & B_{28} & B_{29} \\
B_{31} & B_{32} & B_{33} & B_{34} & B_{35} & B_{36} & B_{37} & B_{38} & B_{39}
\end{array}\right]
\end{aligned}
$$

Com

$$
\begin{aligned}
& X B U=b_{1} u_{1}+b_{2} u_{2}+b_{3} u_{3}, \quad X B V=b_{1} v_{1}+b_{2} v_{2}+b_{3} v_{3}, \quad X B W=b_{1} w_{1}+b_{2} w_{2}+b_{3} w_{3}, \\
& X C U=c_{1} u_{1}+c_{2} u_{2}+c_{3} u_{3}, \quad X C V=c_{1} v_{1}+c_{2} v_{2}+c_{3} v_{3}, \quad X C W=c_{1} w_{1}+c_{2} w_{2}+c_{3} w_{3},
\end{aligned}
$$

Das equações (5.26) e (5.27),

$$
\begin{aligned}
& B_{11}=(X B U) b_{1} \quad B_{21}=(X C U) c_{1} \quad B_{31}=(X B U) c_{1} \\
& B_{12}=(X B V) b_{1} \quad B_{22}=(X C V) c_{1} \quad B_{32}=(X B V) c_{1} \\
& B_{13}=(X B W) b_{1} \quad B_{23}=(X C W) c_{1} \quad B_{33}=(X B W) c_{1} \\
& B_{14}=(X B U) b_{2} \quad B_{24}=(X C U) c_{2} \quad B_{34}=(X B U) c_{2} \\
& B_{15}=(X B V) b_{2} \quad B_{25}=(X C V) c_{2} \quad B_{35}=(X B V) c_{2} \\
& B_{16}=(X B W) b_{2} \quad B_{26}=(X C W) c_{2} \quad B_{36}=(X B W) c_{2} \\
& B_{17}=(X B U) b_{3} \quad B_{27}=(X C U) c_{3} \quad B_{37}=(X B U) c_{3} \\
& B_{18}=(X B V) b_{3} \quad B_{28}=(X C V) c_{3} \quad B_{38}=(X B V) c_{3} \\
& B_{19}=(X B W) b_{3} \quad B_{29}=(X C W) c_{3} \quad B_{39}=(X B W) c_{3}
\end{aligned}
$$


A discretização feita utilizando as equações de Hill (1950) a seguir:

$$
\begin{aligned}
d \bar{E} & =\frac{(1+R)}{\sqrt{1+2 R}} \sqrt{d E_{x}^{2}+d E_{y}^{2}+\frac{2 R}{1+R} d E_{x} d E_{y}+\frac{2}{1+R} d E_{x y}^{2}}= \\
d \bar{E} & =\sqrt{\frac{2}{3} d \mathbf{E}^{T} \mathbf{D} d \mathbf{E}}
\end{aligned}
$$

e a equação (5.30).

Para a equação (5.33), tomando a variação com relação ao vetor deslocamento u, chega-se a:

$$
\delta(d \bar{E})=\frac{2}{3}\left(d \bar{E}^{-1}\right) d \mathbf{E}^{T} \mathbf{D} \delta(d \mathbf{E})
$$

e da equação (5.30),

$$
\delta(d \mathbf{E})=\left(\mathbf{B}+\mathbf{B}_{, \mathbf{u}} \mathbf{u}\right) \delta \mathbf{u}
$$

onde $B_{, u} \mathbf{u}$ é uma matriz $3 \times 9$.

A equação (5.17), para a discretização de cada elemento ficará:

$$
\delta \phi_{(m)}=\int_{V} \frac{2}{3} \frac{\bar{S}}{d \bar{E}} \delta \mathbf{u}^{T}\left(\mathbf{B}+\mathbf{B}_{, u} \mathbf{u}\right)^{T} \mathbf{D B} \mathbf{u} d V-\int_{S} \delta \mathbf{u}^{T} \mathbf{N}^{T} \mathbf{f} d S
$$

que ainda deverá ser transformada para o sistema de coordenadas globais.

A discretização para Hill (1979) fica (usando 3.70)

$$
\begin{aligned}
& \delta(\mathrm{d} \bar{E})=\frac{1}{M}\left(2^{1 / M-1}(M-1)(1+R)^{1 / M}\left(\left(d \mathbf{E}^{T} \mathbf{D}_{H 1} d \mathbf{E}\right)^{\frac{M}{2(M-1)}}+(1+2 R)^{\frac{1}{M-1}}\left(d \mathbf{E}^{T} \mathbf{D}_{H 2} d \mathbf{E}\right)^{\frac{M}{2(M-1)}}\right)^{\frac{M-1}{M}-1}\right. \\
& \left.\frac{M}{M-1}\left(\left(d \mathbf{E}^{T} \mathbf{D}_{H 1} d \mathbf{E}\right)^{\frac{M}{2(M-1)}-1} d \mathbf{E}^{T} \mathbf{D}_{H 1} \delta(d \mathbf{E})+(1+2 R)^{\frac{1}{M-1}}\left(d \mathbf{E}^{T} \mathbf{D}_{H 2} d \mathbf{E}\right)^{\frac{M}{2(M-1)}-1} d \mathbf{E}^{T} \mathbf{D}_{H 2} \delta(d \mathbf{E})\right)\right)
\end{aligned}
$$


onde $\delta(d \mathbf{E})$ é o mesmo de $(5.35)$

$$
\begin{aligned}
& \delta \phi_{(m)}=\int_{v} \bar{S} \frac{1}{M}\left(2 ^ { 1 / M - 1 } ( M - 1 ) ( 1 + R ) ^ { 1 / M } \left(\left(\mathbf{u B D}_{H 1} \mathbf{B u}\right)^{\frac{M}{2(M-1)}}\right.\right. \\
& \left.+(1+2 R)^{\frac{1}{M-1}}\left(\mathbf{u} \mathbf{B D}_{H 2} \mathbf{B u}\right)^{\frac{M}{2(M-1)}}\right)^{\frac{M-1}{M}-1} \\
& \frac{M}{M-1}\left(\left(\mathbf{u B D}{ }_{H 1} \mathbf{B u}\right)^{\frac{M}{2(M-1)}-1} \delta \mathbf{u}^{T}\left(\mathbf{B}+\mathbf{B}_{, u} \mathbf{u}\right)^{T} \mathbf{D}_{H 1} \mathbf{B u}\right. \\
& \left.\left.+(1+2 R)^{\frac{1}{M-1}}\left(\mathbf{u B D}_{H 2} \mathbf{B} \mathbf{u}\right)^{\frac{M}{2(M-1)}-1} \delta \mathbf{u}^{T}\left(\mathbf{B}+\mathbf{B}_{, u} \mathbf{u}\right)^{T} \mathbf{D}_{H 2} \mathbf{B u}\right)\right) d V \\
& -\int_{S} \delta \mathbf{u}^{T} \mathbf{N}^{T} \mathbf{f} d S
\end{aligned}
$$

A discretização para Barlat et al. (1993) fica (usando 3.82)

$$
\delta(\mathrm{d} \bar{E})=\frac{1}{k \zeta}\left(2^{-1-\zeta} 3^{-\zeta}(k \mathrm{~d} \bar{E})^{1-\zeta}\right)\left(\mathbf{T}_{1}+\mathbf{T}_{2}+\mathbf{T}_{3}\right)
$$

onde

$$
\begin{aligned}
& \mathbf{T}_{1}=2^{\zeta} \mathbf{D}_{B 1} d \mathbf{E}\left|\mathbf{D}_{B 1} d \mathbf{E}\right|^{\zeta-2} \mathbf{D}_{B 1} \delta(d \mathbf{E}) \\
& \mathbf{T}_{2}=\zeta\left(\mathbf{D}_{B 1} d \mathbf{E}-\sqrt{d \mathbf{E}^{T} \mathbf{D}_{B} d \mathbf{E}}\right)\left|\mathbf{D}_{B 1} d \mathbf{E}-\sqrt{d \mathbf{E}^{T} \mathbf{D}_{B} d \mathbf{E}}\right|^{\zeta-2}\left(\mathbf{D}_{B 1} \delta(d \mathbf{E})-\frac{d \mathbf{E}^{T} \mathbf{D}_{B} \delta(d \mathbf{E})}{\sqrt{d \mathbf{E}^{T} \mathbf{D}_{B} d \mathbf{E}}}\right) \\
& \mathbf{T}_{3}=\zeta\left(\mathbf{D}_{B 1} d \mathbf{E}+\sqrt{d \mathbf{E}^{T} \mathbf{D}_{B} d \mathbf{E}}\right)\left|\mathbf{D}_{B 1} d \mathbf{E}+\sqrt{d \mathbf{E}^{T} \mathbf{D}_{B} d \mathbf{E}}\right|^{\zeta-2}\left(\mathbf{D}_{B 1} \delta(d \mathbf{E})+\frac{d \mathbf{E}^{T} \mathbf{D}_{B} \delta(d \mathbf{E})}{\sqrt{d \mathbf{E}^{T} \mathbf{D}_{B} d \mathbf{E}}}\right)
\end{aligned}
$$

A equação (5.39) não é muito difícil de ser implementada, porém o critério de Barlat et al. (1993) é anisotrópico planar e necessita de um estudo da orientação dos eixos de anisotropia após cada deformação. Considerando um elemento em deformação, temos o gradiente de deformação $\Delta \mathbf{F}$, que pode ser decomposto em um produto de dois tensores, o tensor rotação $\Delta \mathbf{R}$ e o tensor de estiramento $\Delta \mathbf{R}$ :

$$
\Delta \mathbf{F}=\Delta \mathbf{R} \cdot \Delta \mathbf{U}
$$

Inicialmente os eixos de anisotropia estão alinhados com o eixo de coordenada cartesiano global. Durante a deformação $\Delta \mathbf{F}$, a superfície de escoamento rotaciona $\Delta \mathbf{R}$ e então os eixos de anisotropia não estão mais alinhados com o eixo de coordenada global depois do primeiro incremento. Então é necessário traçar a rotação dos eixos de anisotropia 


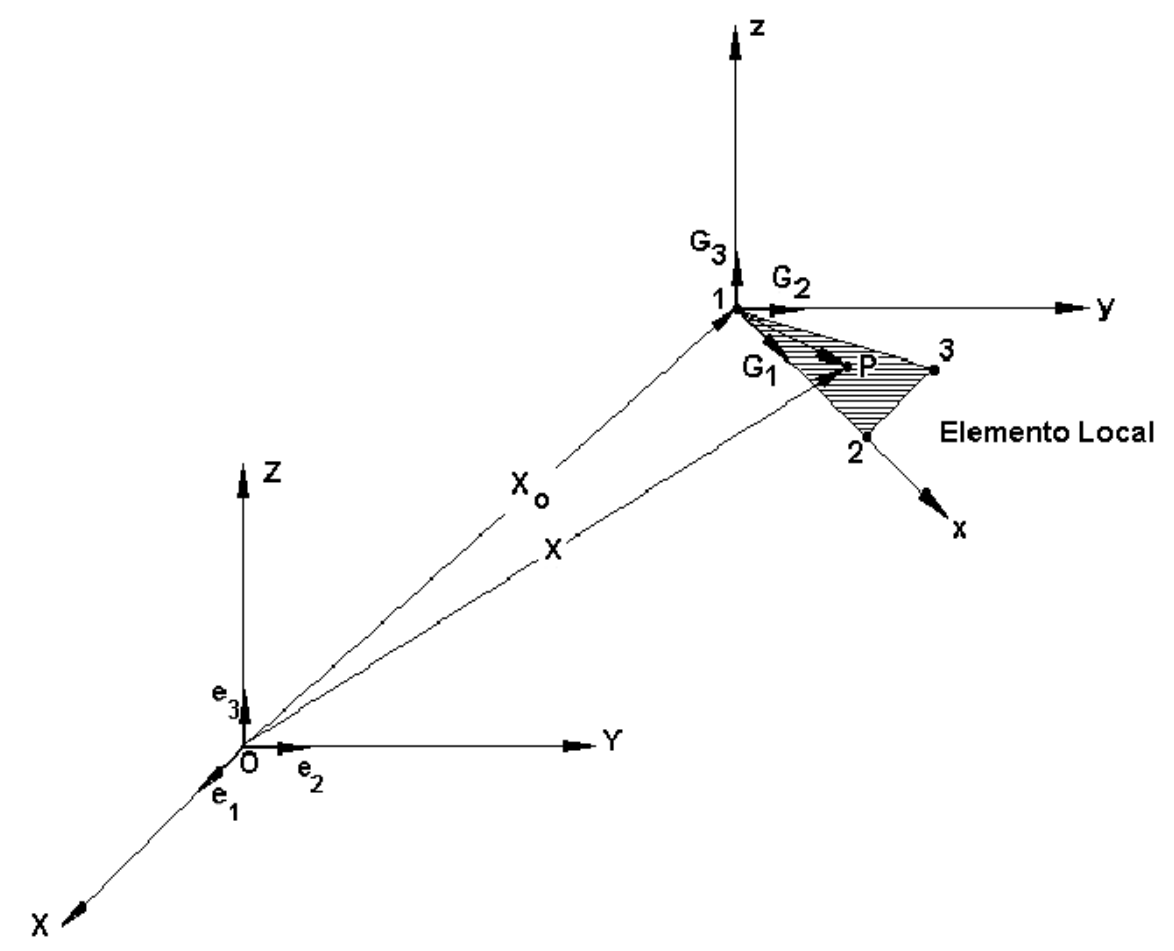

Figura 37: Sistemas de coordenadas Cartesianas global e local (Toh (1983)).

durante a deformação para expressar o incremento de deformação, equação (5.39), nas coordenadas locais. Para isotropia planar, o caso do programa SHEET que usa o critério de Hill (1950), este procedimento não é necessário, como será visto na seção 5.4, onde as coordenadas locais estão alinhadas com as coordenadas dos nós dos elementos, diferente de Barlat et al. (1993) em que as coordenadas locais devem estar alinhadas com os eixos de anisotropia.

\subsection{Translação de Coordenadas}

A partir da figura $37, \operatorname{com}(\mathrm{X}, \mathrm{Y}, \mathrm{Z})$ o vetor base $\mathbf{e}_{\mathbf{i}},(x, y, z)$, base do vetor $\mathbf{G}_{\mathbf{i}}$ onde $i=1,2,3$, a transformação do sistema de coordenadas local para o global, será feita a partir da seguinte formulação:

$$
\mathbf{x}=\boldsymbol{\lambda}\left(\mathrm{X}-\mathrm{X}_{\mathbf{0}}\right)
$$


onde:

$\mathbf{x}$ é o vetor posição de ponto arbitrário $\mathrm{p}$ referido ao sistema $(x, y, z)$,

$\mathbf{X}$ é o vetor posição do ponto referido ao sistema $(X, Y, Z)$,

$\mathbf{X}_{\mathbf{0}}$ denota a posição do sistema $(\mathrm{x}, \mathrm{y}, \mathrm{z})$ com relação ao sistema $(X, Y, Z)$,

e $\boldsymbol{\lambda}$ é a matriz de tranformação consistindo dos cosenos diretores:

$$
\begin{gathered}
\boldsymbol{\Lambda}=\left[\begin{array}{ccc}
G_{1} \cdot e_{1} & G_{1} \cdot e_{2} & G_{1} \cdot e_{3} \\
G_{2} \cdot e_{1} & G_{2} \cdot e_{2} & G_{2} \cdot e_{3} \\
G_{3} \cdot e_{1} & G_{3} \cdot e_{2} & G_{3} \cdot e_{3}
\end{array}\right] \\
\{\mathbf{u}\}=\lambda\{U\}
\end{gathered}
$$

onde $\mathbf{u}$ e $\mathbf{U}$ são os valores de incremento de deslocamento locais e globais, respectivamente. A matriz $\Lambda$ é 9x9, e representada por

$$
\Lambda=\left[\begin{array}{lll}
\lambda & \lambda & \lambda \\
\lambda & \lambda & \lambda \\
\lambda & \lambda & \lambda
\end{array}\right]
$$

Deste modo, a equação (5.36) (Hill (1950)) é transformada para um sitema global de coordenadas via equação (5.44).

Assim,

$$
\psi^{(m)}=\delta \phi_{(m)}=\int_{v} \frac{2}{3} \frac{\bar{S}}{d \bar{E}} \delta \mathbf{U}^{T} \boldsymbol{\Lambda}^{T} \mathbf{Q}^{T} \mathbf{D} \mathbf{B} \boldsymbol{\Lambda} \mathbf{U} d V-\int_{S} \delta \mathbf{U}^{T} \boldsymbol{\Lambda}^{T} \mathbf{N}^{T} \mathbf{f} d S
$$

com $\mathbf{Q}=\mathbf{B}+\mathbf{B}_{, u} \mathbf{u}$, com o superescrito $m$ associado ao m-ésimo elemento. 
E do mesmo modo a equação (5.38) de Hill (1979) fica

$$
\begin{aligned}
& \delta \phi_{(m)}=\int_{v} \bar{S} \frac{1}{M}\left(2 ^ { 1 / M - 1 } ( M - 1 ) ( 1 + R ) ^ { 1 / M } \left(\left(\mathbf{U}^{T} \boldsymbol{\Lambda}^{T} \mathbf{B}^{T} \mathbf{D}_{H 1} \mathbf{B} \boldsymbol{\Lambda} \mathbf{U}\right)^{\frac{M}{2(M-1)}}+\right.\right. \\
& \left.(1+2 R)^{\frac{1}{M-1}}\left(\mathbf{U}^{T} \boldsymbol{\Lambda}^{T} \mathbf{B}^{T} \mathbf{D}_{H 2} \mathbf{B} \boldsymbol{\Lambda} \mathbf{U}\right)^{\frac{M}{2(M-1)}}\right)^{\frac{M-1}{M}-1} \\
& \frac{M}{M-1}\left(\left(\mathbf{U}^{T} \boldsymbol{\Lambda}^{T} \mathbf{B}^{T} \mathbf{D}_{H 1} \mathbf{B} \boldsymbol{\Lambda} \mathbf{U}\right)^{\frac{M}{2(M-1)}-1} \delta \mathbf{U}^{T} \boldsymbol{\Lambda}^{T} \mathbf{Q}^{T} \mathbf{D}_{H 1} \mathbf{B} \boldsymbol{\Lambda} \mathbf{U}+\right. \\
& \left.\left.(1+2 R)^{\frac{1}{M-1}}\left(\mathbf{U} \boldsymbol{\Lambda}^{T} \mathbf{B} \mathbf{D}_{H 2} \mathbf{B} \boldsymbol{\Lambda} \mathbf{U}\right)^{\frac{M}{2(M-1)}-1} \delta \mathbf{U}^{T} \boldsymbol{\Lambda}^{T} \mathbf{Q}^{T} \mathbf{D}_{H 2} \mathbf{B} \boldsymbol{\Lambda} \mathbf{U}\right)\right) \mathrm{d} V \\
& -\int_{S} \delta \mathbf{U}^{T} \mathbf{N}^{T} \mathbf{f} d S
\end{aligned}
$$

\subsection{Método de Solução}

A partir da equação 5.36 é possível, para todo o domínio composto de elementos finitos, definir um sistema de equações simultâneas não linear.

A solução do sistema não linear é então implementada. O método empregado é o denominado de Newton-Raphson por Dahlquist (1974). Em síntese, sendo uma equação não linear $\psi^{(m)}(\mathbf{u})=0$, e $\mathbf{u}^{*}$ é uma solução, a seguinte série é, expandida:

$$
\psi^{(m)}(\mathbf{u})=\psi^{(m)}\left(\mathbf{u}^{*}\right)+\left(\frac{\mathrm{d} \psi^{(m)}}{\mathrm{d} \mathbf{u}}\right)_{u-u^{*}}\left(\mathbf{u}-\mathbf{u}^{*}\right)+\frac{1}{2}\left(\frac{\mathrm{d}^{2} \psi}{\mathrm{d} \mathbf{u}^{2}}\right)_{u-u^{*}}\left(\mathbf{u}-\mathbf{u}^{*}\right)^{2}+\cdots
$$

onde $\psi^{(m)}(\mathbf{u})$ é uma função diferenciável e $\Delta u=u-u^{*}$.

No presente caso, os termos de maior ordem negligenciados, e 5.48 pode ser escrita na forma:

$$
\psi^{(m)} \approx \psi^{(m)^{*}}+\left[\frac{\mathrm{d} \psi^{(m)}}{\mathrm{d} \mathbf{u}}\right] \Delta \mathbf{u}+\cdots
$$

onde, para Hill (1950)

$$
\psi^{(m)^{*}}=\left(\frac{2}{3} \int_{V} \frac{\bar{S}}{\mathrm{~d} \bar{E}} \delta \mathbf{U}^{T} \boldsymbol{\Lambda}^{T} \mathbf{Q}^{T} \mathbf{D} \mathbf{B} \boldsymbol{\Lambda} \mathbf{U d} V-\int_{S} \delta \mathbf{U}^{T} \boldsymbol{\Lambda}^{T} \mathbf{N}^{T} \mathbf{f}^{T} \mathrm{~d} S\right)^{*}
$$

e

$$
\left[\frac{\mathrm{d} \psi^{(m)}}{\mathrm{d} \mathbf{U}}\right]^{*}=\left(\frac{\mathrm{d}}{\mathrm{d} \mathbf{u}}\left\{\frac{2}{3} \int_{V} \frac{\bar{S}}{\mathrm{~d} \bar{E}} \delta \mathbf{u}^{T} \mathbf{Q}^{T} \mathbf{D B} \mathbf{u d} V-\int_{S} \delta \mathbf{u}^{T} \mathbf{N}^{T} \mathbf{f}^{T} \mathrm{~d} S\right\} \frac{\mathrm{d} \mathbf{u}}{\mathrm{d} \mathbf{U}}\right)^{*}
$$


e para Hill (1979)

$$
\begin{aligned}
& \psi^{(m)^{*}}=\left(\int _ { V } \overline { S } \frac { 1 } { M } \left(2 ^ { 1 / M - 1 } ( M - 1 ) ( 1 + R ) ^ { 1 / M } \left(\left(\mathbf{U}^{T} \boldsymbol{\Lambda}^{T} \mathbf{B}^{T} \mathbf{D}_{H 1} \mathbf{B} \boldsymbol{\Lambda} \mathbf{U}\right)^{\frac{M}{2(M-1)}}+\right.\right.\right. \\
& \left.(1+2 R)^{\frac{1}{M-1}}\left(\mathbf{U}^{T} \boldsymbol{\Lambda}^{T} \mathbf{B}^{T} \mathbf{D}_{H 2} \mathbf{B} \boldsymbol{\Lambda} \mathbf{U}\right)^{\frac{M}{2(M-1)}}\right)^{\frac{M-1}{M}-1} \\
& \frac{M}{M-1}\left(\left(\mathbf{U}^{T} \boldsymbol{\Lambda}^{T} \mathbf{B}^{T} \mathbf{D}_{H 1} \mathbf{B} \boldsymbol{\Lambda} \mathbf{U}\right)^{\frac{M}{2(M-1)}-1} \delta \mathbf{U}^{T} \boldsymbol{\Lambda}^{T} \mathbf{Q}^{T} \mathbf{D}_{H 1} \mathbf{B} \boldsymbol{\Lambda} \mathbf{U}+\right. \\
& \left.\left.\left.(1+2 R)^{\frac{1}{M-1}}\left(\mathbf{U} \boldsymbol{\Lambda}^{T} \mathbf{B D}_{H 2} \mathbf{B} \boldsymbol{\Lambda} \mathbf{U}\right)^{\frac{M}{2(M-1)}}-1 \delta \mathbf{U}^{T} \boldsymbol{\Lambda}^{T} \mathbf{Q}^{T} \mathbf{D}_{H 2} \mathbf{B} \boldsymbol{\Lambda} \mathbf{U}\right)\right) \mathrm{d} V-\int_{S} \delta \mathbf{U}^{T} \boldsymbol{\Lambda}^{T} \mathbf{N}^{T} \mathbf{f}^{T} \mathrm{~d} S\right)^{*}
\end{aligned}
$$

e

$$
\begin{aligned}
& {\left[\frac{\mathrm{d} \psi^{(m)}}{\mathrm{d} \mathbf{U}}\right]^{*}=\left(\frac { \mathrm { d } } { \mathrm { d } \mathbf { u } } \left\{\int _ { V } \overline { S } \frac { 1 } { M } \left(2 ^ { 1 / M - 1 } ( M - 1 ) ( 1 + R ) ^ { 1 / M } \left(\left(\mathbf{u}^{T} \mathbf{B}^{T} \mathbf{D}_{H 1} \mathbf{B u}\right)^{\frac{M}{2(M-1)}}+\right.\right.\right.\right.} \\
& \left.(1+2 R)^{\frac{1}{M-1}}\left(\mathbf{u}^{T} \mathbf{B}^{T} \mathbf{D}_{H 2} \mathbf{B u}\right)^{\frac{M}{2(M-1)}}\right)^{\frac{M-1}{M}-1} \frac{M}{M-1}\left(\left(\mathbf{u}^{T} \mathbf{B}^{T} \mathbf{D}_{H 1} \mathbf{B u}\right)^{\frac{M}{2(M-1)}-1} \delta \mathbf{u}^{T} \mathbf{Q}^{T} \mathbf{D}_{H 1} \mathbf{B u}+\right. \\
& \left.\left.\left.\left.(1+2 R)^{\frac{1}{M-1}}\left(\mathbf{u B D}_{H 2} \mathbf{B u}\right)^{\frac{M}{2(M-1)}-1} \delta \mathbf{u}^{T} \mathbf{Q}^{T} \mathbf{D}_{H 2} \mathbf{B u}\right)\right) \mathrm{d} V-\int_{S} \delta \mathbf{u}^{T} \mathbf{N}^{T} \mathbf{f}^{T} \mathrm{~d} S\right\} \frac{\mathrm{d} \mathbf{u}}{\mathrm{d} \mathbf{U}}\right)^{*}
\end{aligned}
$$

A equação 5.49 ainda pode ser escrita na forma:

$$
\sum_{m=1}^{N}\left[\psi^{(m)}\left(\mathbf{U}^{*}\right)\right]+\sum_{m=1}^{N}\left[\frac{\mathrm{d} \psi^{(m)}}{\mathrm{d} \mathbf{U}}\right]^{*} \Delta \mathbf{U}=0
$$

ou ainda

$$
\mathbf{P}^{*} \Delta \mathbf{U}=\mathbf{F}-\mathbf{H}^{*}
$$

onde $\mathbf{P}^{*}$ é a matriz de rigidez total,

$$
\mathbf{P}^{*}=\left[\frac{\mathrm{d} \psi^{(m)}}{\mathrm{d} \mathbf{U}}\right]^{*}
$$

$\mathbf{F}-\mathbf{H}^{*}$ o vetor de carga, onde

$$
\mathbf{F}=\int_{S} \boldsymbol{\Lambda}^{T} \mathbf{N}^{T} \mathbf{f}^{T} \mathrm{~d} S
$$

e para Hill (1950)

$$
\mathbf{H}^{*}=\frac{2}{3} \int_{V} \frac{\bar{S}}{\mathrm{~d} \bar{E}} \boldsymbol{\Lambda}^{T} \mathbf{Q}^{T} \mathbf{B} \boldsymbol{\Lambda} \mathbf{U d} V
$$


e para Hill (1979)

$$
\begin{aligned}
& \mathbf{H}^{*}=\int_{V} \bar{S} \frac{1}{M}\left(2 ^ { 1 / M - 1 } ( M - 1 ) ( 1 + R ) ^ { 1 / M } \left(\left(\mathbf{U}^{T} \boldsymbol{\Lambda}^{T} \mathbf{B}^{T} \mathbf{D}_{H 1} \mathbf{B} \boldsymbol{\Lambda} \mathbf{U}\right)^{\frac{M}{2(M-1)}}+\right.\right. \\
& \left.(1+2 R)^{\frac{1}{M-1}}\left(\mathbf{U}^{T} \boldsymbol{\Lambda}^{T} \mathbf{B}^{T} \mathbf{D}_{H 2} \mathbf{B} \boldsymbol{\Lambda} \mathbf{U}\right)^{\frac{M}{2(M-1)}}\right)^{\frac{M-1}{M}-1} \\
& \frac{M}{M-1}\left(\left(\mathbf{U}^{T} \boldsymbol{\Lambda}^{T} \mathbf{B}^{T} \mathbf{D}_{H 1} \mathbf{B} \boldsymbol{\Lambda} \mathbf{U}\right)^{\frac{M}{2(M-1)}-1} \boldsymbol{\Lambda}^{T} \mathbf{Q}^{T} \mathbf{D}_{H 1} \mathbf{B} \boldsymbol{\Lambda} \mathbf{U}+\right. \\
& \left.\left.(1+2 R)^{\frac{1}{M-1}}\left(\mathbf{U} \boldsymbol{\Lambda}^{T} \mathbf{B D}_{H 2} \mathbf{B} \boldsymbol{\Lambda} \mathbf{U}\right)^{\frac{M}{2(M-1)}-1} \boldsymbol{\Lambda}^{T} \mathbf{Q}^{T} \mathbf{D}_{H 2} \mathbf{B} \boldsymbol{\Lambda} \mathbf{U}\right)\right) \mathrm{d} V
\end{aligned}
$$

$\Delta \mathbf{U}$ as pertubações e $N$ é o número total de elementos finitos.

A solução do problema físico é obtida pela introdução das apropriadas condições de contorno em (5.55). Para um incremento de deslocamento prescrito, as correspondentes perturbações deverão se anular e, para uma condição de contorno prescrita para forças de tração, o valor das forças de tração será fornecido através do vetor F. O procedimento de solução é o seguinte:

- Assumir uma estimativa inicial $\mathbf{U}^{(1)}$ e calcular $\mathbf{P}, \mathbf{H}$ e $\mathbf{F}$, correspondente a esta estimativa.

- Resolver a equação 5.55 e obter $\Delta \mathbf{U}$

- Obter uma nova estimativa inicial: $\mathbf{U}^{(2)}=\mathbf{U}^{(1)}+\Delta \mathbf{U}$

Este processo é repetido até que a convergência seja atingida. A convergência é verificada pela norma fracional. Definida

$$
\|\mathbf{U}\|=\sqrt{\mathbf{U}_{1}^{2}+\mathbf{U}_{2}^{2}+\cdots}
$$

e

$$
\|\Delta \mathbf{U}\|=\sqrt{\left(\Delta \mathbf{U}_{1}\right)^{2}+\left(\Delta \mathbf{U}_{2}\right)^{2}+\cdots}
$$

A norma fracional é definida pela razão $\|\mathbf{U}\| /\|\Delta \mathbf{U}\|$ e quando após subsequentes interações, este valor alcança uma magnitude menor que um valor predeterminado, $\left(10^{-5}\right.$, por exemplo) o processo interativo para e a solução é obtida. 


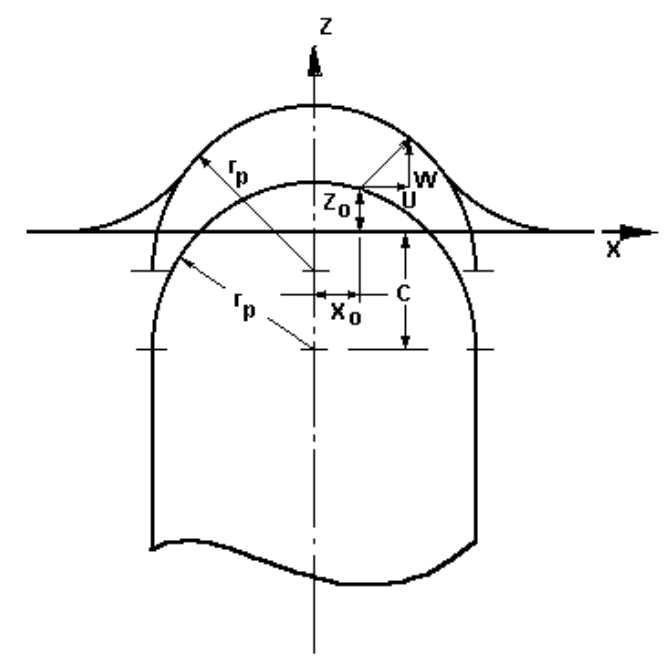

Figura 38: Vista esquemática dos requisitos geometricos para o nó em contato com o punção (Toh (1983)).

\subsection{Condições de Contorno}

A aplicação do presente método de elementos finitos, requer uma solução para a aplicação das condições de contorno, como já citado na seção 5.5.

\subsubsection{Condições de Contato - Punção Hemisférico}

As posições dos elementos materiais do punção e da chapa na região de contato não são independentes, mas se relacionam através da expressão matemática que exprime as condições de contato na superfície da cabeça do punção (figura 38). Esta expressão é:

$$
(X+U)^{2}+(Y+V)^{2}+(C+Z+W)^{2}=r_{p}^{2}
$$

onde $X, Y$ e $Z$ são as posições do elemento na configuração atual; $U, V$ e $W$ são os incrementos de deslocamento, e $C$ é um parâmetro relacionado com a altura do punção $h$ dado pela expressão:

$$
C=r_{p}-h
$$

As condições de contorno na região do prensa-chapas e da matriz são formulados de forma similar ao do punção. Considerando a geometria da matriz com referência às 
coordenadas globais

$$
\left(\sqrt{(X+U)^{2}+(Y+V)^{2}}-a\right)^{2}+\left(Z+W-r_{d}\right)^{2}=r_{p}^{2}
$$

onde $r_{d}$ é o raio da matriz e $a$ é a soma do raio da matriz e o raio da abertura da matriz.

As condições de contorno de tração associadas aos nós em contato com punção são formuladas com referência a figura 39. Considerando-se dois sistemas de coordenadas, $X-Y-Z$ e $X^{\prime}-Y^{\prime}-Z^{\prime}$, e assumido que a força normal atuando no ponto de contato é $F_{n}$, e a força tangencial de fricção correspondente no plano $X^{\prime}-Z^{\prime}$ é $T$, tem-se da figura 39,

$$
F_{t}^{\prime}=\frac{T \cos \theta_{1}}{\cos \left(\theta_{3}-\theta_{2}\right)}
$$

que é a projeção de $F_{t}$ no plano $X-Y$. As componentes da força de fricção tangencial, $F_{t}$, são

$$
\begin{aligned}
& F_{t_{x}}=F_{t}^{\prime} \cos \theta_{3}=\frac{\cos \theta_{1} \cos \theta_{3}}{\cos \left(\theta_{3}-\theta_{2}\right)} T \\
& F_{t_{y}}=F_{t}^{\prime} \operatorname{sen} \theta_{3}=\frac{\cos \theta_{1} \operatorname{sen} \theta_{3}}{\cos \left(\theta_{3}-\theta_{2}\right)} T \\
& F_{t_{z}}=\operatorname{sen} \theta_{1} T
\end{aligned}
$$

Então pode-se obter $F_{t}$ como

$$
F_{t}=\sqrt{\left(\frac{\cos \theta_{1} \cos \theta_{3}}{\cos \left(\theta_{3}-\theta_{2}\right)}\right)^{2}+\left(\frac{\cos \theta_{1} \operatorname{sen} \theta_{3}}{\cos \left(\theta_{3}-\theta_{2}\right)}\right)^{2}+\operatorname{sen}^{2} \theta_{1} T}
$$

e fazendo

$$
A=\sqrt{\left(\frac{\cos \theta_{1} \cos \theta_{3}}{\cos \left(\theta_{3}-\theta_{2}\right)}\right)^{2}+\left(\frac{\cos \theta_{1} \operatorname{sen} \theta_{3}}{\cos \left(\theta_{3}-\theta_{2}\right)}\right)^{2}+\operatorname{sen}^{2} \theta_{1}}
$$

então

$$
T=\frac{F_{t}}{A}
$$

Combinando as equações (5.66) e (5.68), junto com a geometria mostrada na figura 

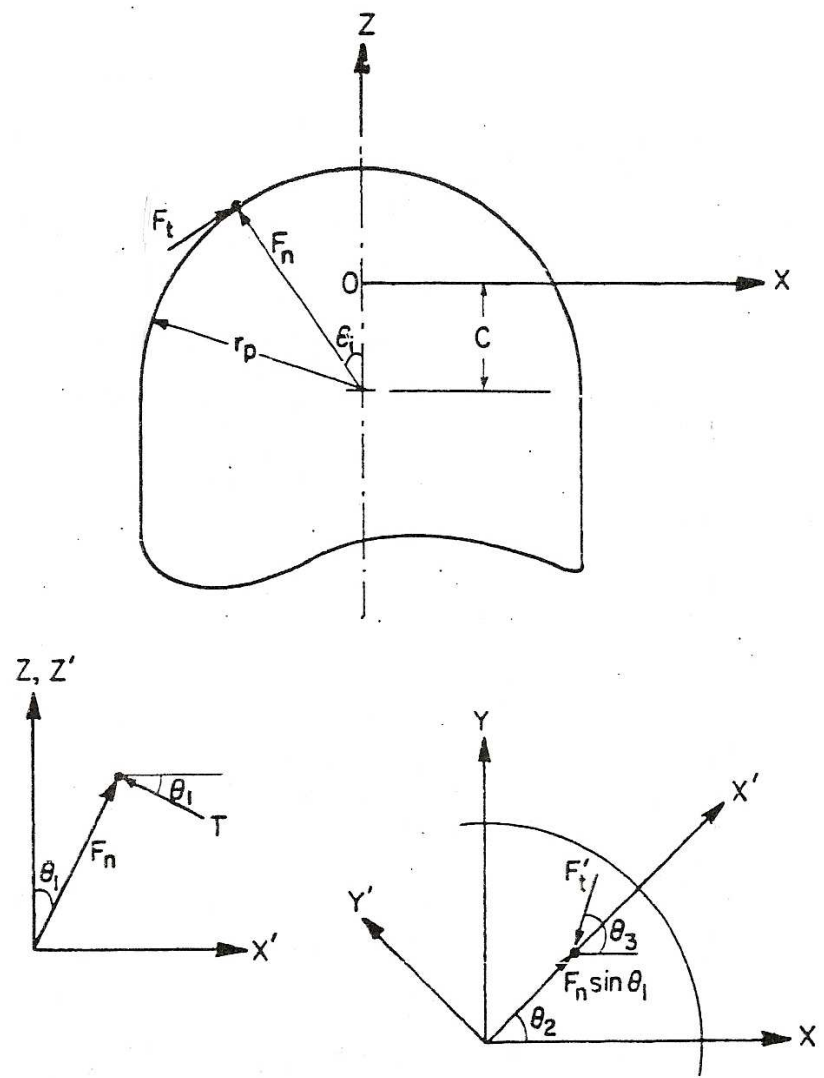

Figura 39: Condição de contorno para o nó em contato com o punção (Toh (1983)).

39, a componente de força no nó associada com o ponto de contato são

$$
\begin{aligned}
& F_{X}=F_{n} \operatorname{sen} \theta_{1} \cos \theta_{2}-\frac{\cos \theta_{1} \cos \theta_{3}}{A \cos \left(\theta_{3}-\theta_{2}\right)} F_{t} \\
& F_{Y}=F_{n} \operatorname{sen} \theta_{1} \operatorname{sen} \theta_{2}-\frac{\cos \theta_{1} \operatorname{sen} \theta_{3}}{A \cos \left(\theta_{3}-\theta_{2}\right)} F_{t} \\
& F_{Z}=F_{n} \cos \theta_{1}+\frac{\operatorname{sen} \theta_{1}}{A} F_{t}
\end{aligned}
$$

onde

$$
\begin{aligned}
\operatorname{sen} \theta_{1} & =\frac{\sqrt{(X+U)^{2}+(Y+V)^{2}}}{r_{p}} \\
\cos \theta_{1} & =\frac{(Z+W+C)}{r_{p}} \\
\operatorname{sen} \theta_{2} & =\frac{(Y+V)}{\sqrt{(X+U)^{2}+(Y+V)^{2}}} \\
\cos \theta_{2} & =\frac{(X+U)}{\sqrt{(X+U)^{2}+(Y+V)^{2}}} \\
\operatorname{sen} \theta_{3} & =\frac{V}{\sqrt{U^{2}+V^{2}}} \\
\cos \theta_{3} & =\frac{U}{\sqrt{U^{2}+V^{2}}}
\end{aligned}
$$




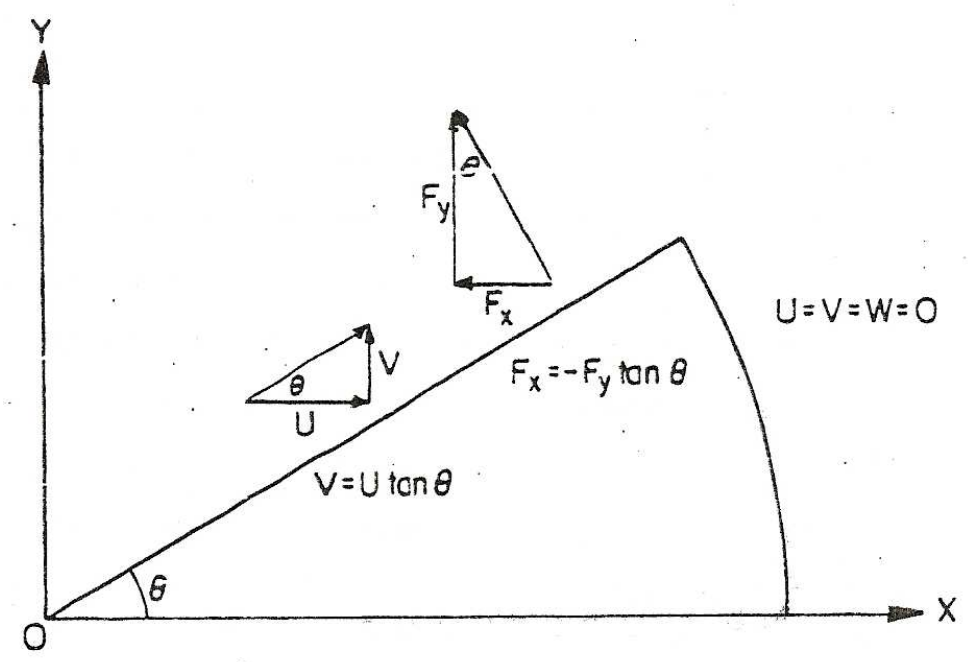

Figura 40: Condições de contorno para o estiramento (Toh (1983)).

A equação (5.71) é prescrita no vetor de carga, F, quando um nó entra em contato com o punção. Na presente formulação, a força de atrito, $F_{t}$, é igual a $\mu F_{n}$ onde $\mu$ é o coeficiente de atrito. As condições de contorno de tração para os nós em contato com a matriz são formuladas de modo similar aos nós em contato com o punção. Para reproduzir a força do prensa-chapas, ela é tratada como uma força de atrito tangencial concentrada nos nós localizados no contorno do blank. Alguns detalhes das condições de contorno para o estiramento podem ser encontrados na figura 40 e para a estampagem na figura 41 . A força no punção é calculada somando-se as forças $F_{n}$ na direção $Z$, de todos os nós em contato com o punção.

Com o avanço do punção, a porção da chapa em contato com o punção aumenta e os nós localizados na região do prensa-chapas se movem pela matriz, mudam-se as condições de contorno a cada passo. A técnica consiste em assumir a posição do contorno na próxima configuração, para determinar quais nós estão em contato com o punção e a matriz. Obtém-se a convergência da solução para esta situação e verifica-se se isto é verdadeiro. Ou seja, a abordagem é do tipo tentativa e erro. 


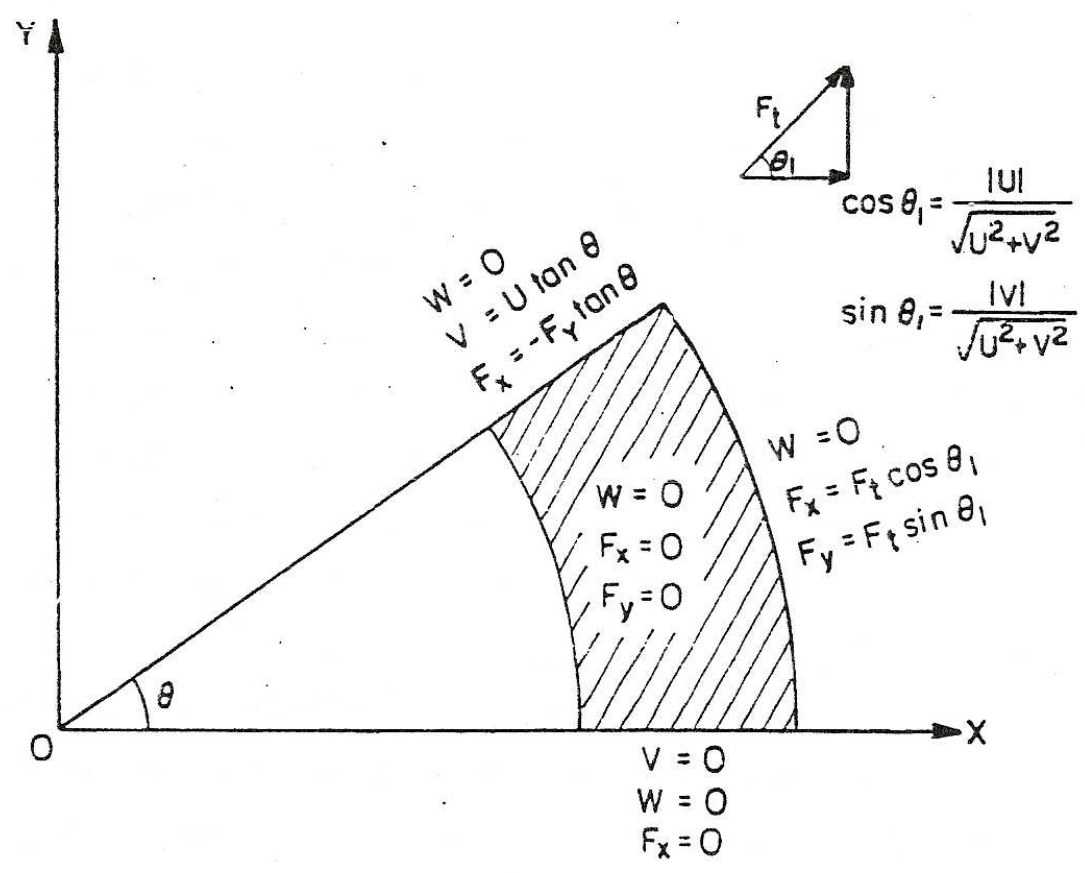

Figura 41: Condições de contorno para a parte da flange na estampagem profunda. Área hachurada indica a região da flange (Toh (1983)).

\subsubsection{Condições de Contato - Punção Quadrado}

Na estampagem de copos quadrados, as condições de contorno são mais complicadas que na estampagem com punção hemisférico. As condições de contato punção-chapa são formuladas separadamente de acordo com as relações geométricas. Com referência as figuras 42 e 43 as condições de contato com o punção são:

Na parte plana do punção:

$$
\left(X+U-\frac{a}{2}+r_{p}\right)^{2}+V^{2}+(z+W+C)^{2}=r_{p}^{2}
$$

onde $C=r_{p}-h, h$ sendo a profundidade do punção na configuração atual, $U, V$ e $W$ os deslocamentos globais incrementais nas direções $X, Y$ e $Z$, respectivamente.

No canto do punção:

$$
\left(X+U-\frac{a}{2}+r_{c}+\left(r_{p}-r_{c}\right) \cos \theta\right)^{2}+\left(Y+V-\frac{a}{2}+r_{c}+\left(r_{p}-r_{c}\right) \operatorname{sen} \theta\right)^{2}+(Z+W+C)^{2}=r_{p}^{2}
$$



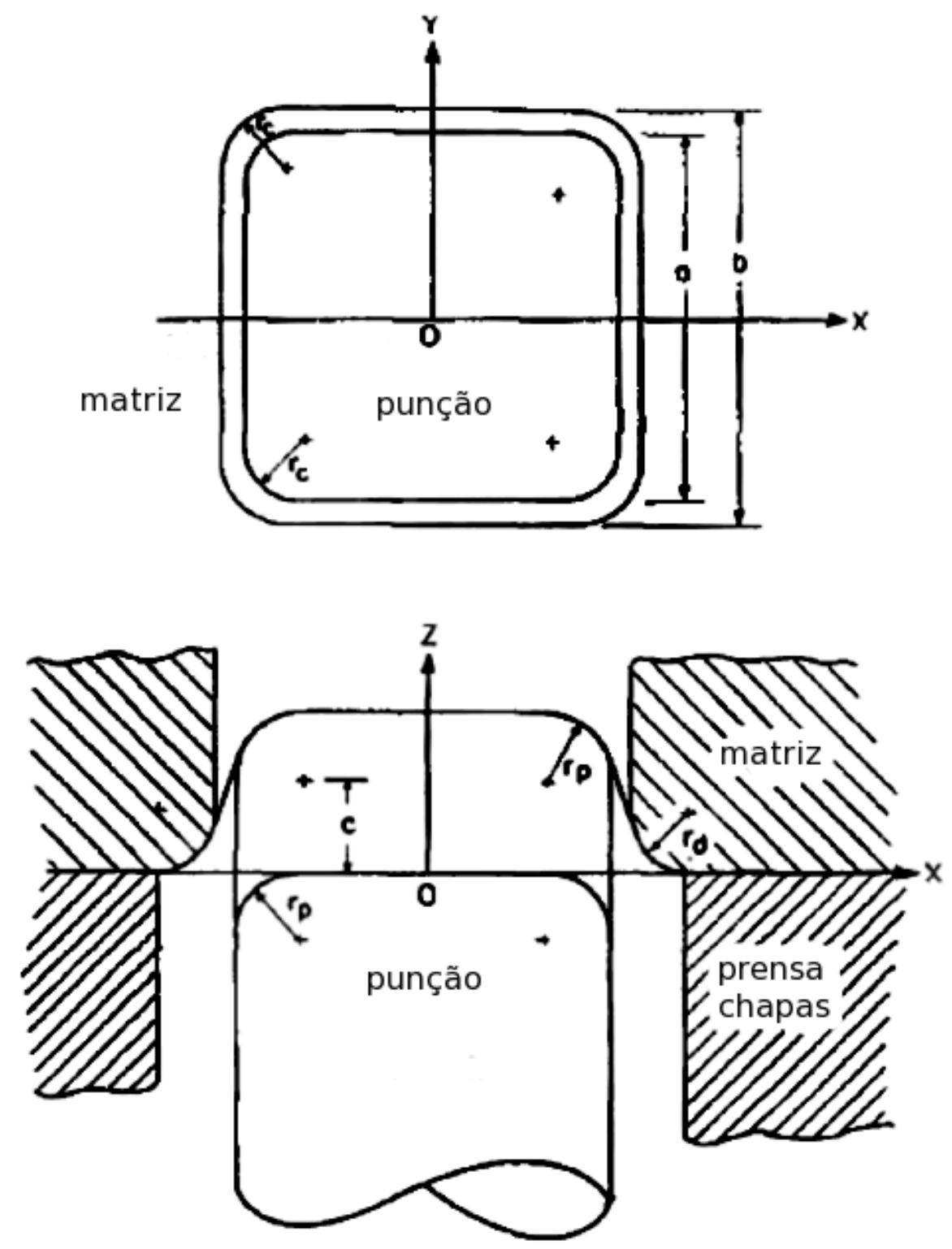

Figura 42: Vista esquemática da estampagem profunda com copo quadrado. Toh (1983)

onde

$$
\cos \theta=\frac{X+U-a / 2+r_{c}}{\sqrt{\left(X+U-\frac{a}{2}+r_{c}\right)^{2}+\left(Y+V-\frac{a}{2}+r_{c}\right)^{2}}}
$$

e

$$
\operatorname{sen} \theta=\frac{Y+V-a / 2+r_{c}}{\sqrt{\left(X+U-\frac{a}{2}+r_{c}\right)^{2}+\left(Y+V-\frac{a}{2}+r_{c}\right)^{2}}}
$$

De forma similar, tem-se as seguintes relações geométricas para a matriz:

Na parte plana da matriz:

$$
\left(X+U-\frac{b}{2}-r_{d}\right)^{2}+V^{2}+\left(Z+W-r_{d}\right)^{2}=r_{d}^{2}
$$




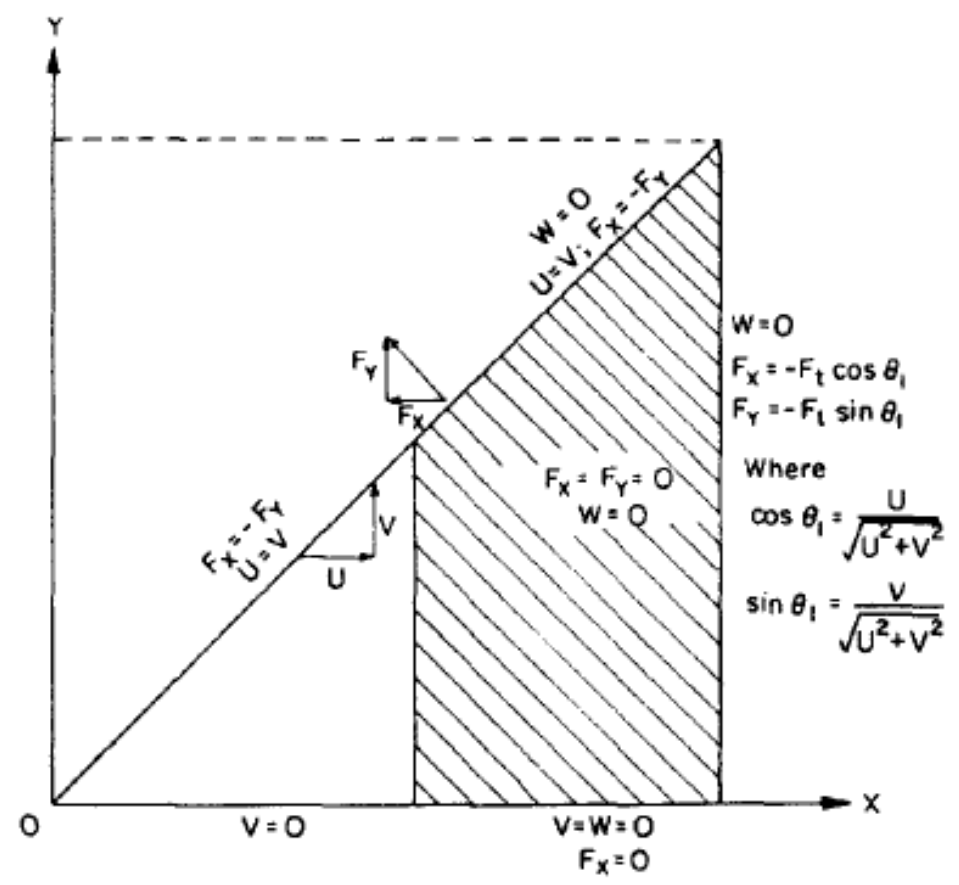

Figura 43: Vista das condições de contorno em uma chapa quadrada antes da deformação. Toh (1983)

No canto da matriz:

$$
\left(X+U-\frac{b}{2}+r_{c}-\left(r_{d}+r_{c}\right) \cos \theta\right)^{2}+\left(Y+V-\frac{b}{2}+r_{c}-\left(r_{d}+r_{c}\right) \operatorname{sen} \theta\right)^{2}+\left(Z+W-r_{d}\right)^{2}=r_{d}^{2}
$$

onde

$$
\cos \theta=\frac{X+U-b / 2+r_{c}}{\sqrt{\left(X+U-\frac{b}{2}+r_{c}\right)^{2}+\left(Y+V-\frac{b}{2}+r_{c}\right)^{2}}}
$$

$\mathrm{e}$

$$
\operatorname{sen} \theta=\frac{Y+V-b / 2+r_{c}}{\sqrt{\left(X+U-\frac{b}{2}+r_{c}\right)^{2}+\left(Y+V-\frac{b}{2}+r_{c}\right)^{2}}}
$$

As condições de contorno de tração para os nós em contato com a parte plana do punção são:

$$
\begin{aligned}
& F_{X}=-\mu F_{Z} \cos \theta_{2} \\
& F_{Y}=-\mu F_{Z} \operatorname{sen} \theta_{2}
\end{aligned}
$$

onde

$$
\begin{aligned}
\cos \theta_{2} & =\frac{U^{*}}{\sqrt{U^{*^{2}}+V^{*^{2}}}} \\
\operatorname{sen} \theta_{2} & =\frac{V^{*}}{\sqrt{U^{*^{2}}+V^{*^{2}}}}
\end{aligned}
$$


onde $\left(^{*}\right)$ denota valores pertubados.

Para os nós em contato com o raio do punção ou o raio da matriz, as condições de contorno seguem o mesmo procedimento feito para o punção esférico.

Na parte reta do raio do punção

$$
\begin{aligned}
& F_{X}=F_{n} \operatorname{sen} \theta_{1}-\frac{F_{t}}{B} \cos \theta_{1} \\
& F_{Y}=\frac{-F_{t}}{B} \cos \theta_{1} \tan \theta_{3} \\
& F_{Z}=F_{n} \cos \theta_{1}+\frac{F_{t}}{B} \operatorname{sen} \theta_{1}
\end{aligned}
$$

onde

$$
\begin{aligned}
& \cos \theta_{1}=\frac{r_{p}+Z+W^{*}-h}{r_{p}} \\
& \operatorname{sen} \theta_{1}=\frac{X+U^{*}-a / 2+r_{p}}{r_{p}} \\
& \tan \theta_{3}=\frac{V^{*}}{U^{*}} \\
& B=\sqrt{1+\left(\cos \theta_{1} \tan _{3}\right)^{2}}
\end{aligned}
$$

No canto do raio do punção

$$
\begin{aligned}
& F_{X}=\left(\operatorname{sen} \theta_{1} \cos \theta_{2}-\frac{\mu \cos \theta_{1} \cos \theta_{3}}{B_{1} \cos \left(\theta_{3}-\theta_{2}\right)}\right) F_{n} \\
& F_{Y}=\left(\operatorname{sen} \theta_{1} \operatorname{sen} \theta_{2}-\frac{\mu \cos \theta_{1} \operatorname{sen} \theta_{3}}{B_{1} \cos \left(\theta_{3}-\theta_{2}\right)}\right) F_{n} \\
& F_{Z}=\left(\cos \theta_{1}+\frac{\mu}{B_{1}} \sin \theta_{1}\right) F_{n}
\end{aligned}
$$

onde

$$
\begin{aligned}
& \cos \theta_{1}=\frac{r_{p}+Z+W^{*}-h}{r_{p}} \\
& \operatorname{sen} \theta_{1}=\sqrt{1-\cos ^{2} \theta_{1}} \\
& \cos \theta_{2}=\frac{\left(X+U^{*}-a / 2+r_{c}\right)}{\sqrt{\left(X+U^{*}-a / 2+r_{c}\right)^{2}+\left(Y+V^{*}-a / 2+r_{c}\right)^{2}}} \\
& \operatorname{sen} \theta_{2}=\frac{\left(Y+V^{*}-a / 2+r_{c}\right)}{\sqrt{\left(X+U^{*}-a / 2+r_{c}\right)^{2}+\left(Y+V^{*}-a / 2+r_{c}\right)^{2}}} \\
& \cos \theta_{3}=\frac{U^{*}}{\sqrt{U^{*^{2}+V^{* 2}}}} \\
& \operatorname{sen} \theta_{3}=\frac{V^{*}}{\sqrt{U^{*^{2}}+V^{*^{2}}}} \\
& B_{1}=\sqrt{\operatorname{sen}^{2} \theta_{1}+\frac{\cos ^{2} \theta_{1}}{\cos ^{2}\left(\theta_{3}-\theta_{2}\right)}}
\end{aligned}
$$


No parte reta do raio da matriz

$$
\begin{aligned}
& F_{X}=\left(\frac{\mu}{B_{2}} \cos \theta_{1}-\operatorname{sen} \theta_{1}\right) F_{n} \\
& F_{Y}=\left(\frac{\mu}{B_{2}} \cos \theta_{1} \tan \theta_{3}\right) F_{n} \\
& F_{Z}=-\left(\frac{\mu}{B_{2}} \operatorname{sen} \theta_{1}+\cos \theta_{1}\right) F_{n}
\end{aligned}
$$

onde

$$
\begin{aligned}
& \cos \theta_{1}=\frac{r_{d}-Z-W^{*}}{r_{d}} \\
& \tan \theta_{3}=\frac{V^{*}}{U^{*}} \\
& B_{2}=\sqrt{1+\left(\cos \theta_{1} \tan \theta_{3}\right)^{2}}
\end{aligned}
$$

No canto do raio da matriz

$$
\begin{aligned}
& F_{X}=\left(-\operatorname{sen} \theta_{1} \cos \theta_{2}+\frac{\mu \cos \theta_{1} \cos \theta_{3}}{B_{3} \cos \left(\theta_{3}-\theta_{2}\right)}\right) F_{n} \\
& F_{Y}=\left(-\operatorname{sen} \theta_{1} \operatorname{sen} \theta_{2}+\frac{\mu \cos \theta_{1} \operatorname{sen} \theta_{3}}{B_{3} \cos \left(\theta_{3}-\theta_{2}\right)}\right) F_{n} \\
& F_{Z}=-\left(\cos \theta_{1}+\frac{\mu}{B_{3}} \sin \theta_{1}\right) F_{n}
\end{aligned}
$$

onde

$$
\begin{aligned}
& \cos \theta_{1}=\frac{r_{p}-Z-W^{*}}{r_{p}} \\
& \cos \theta_{2}=\frac{\left(X+U^{*}-b / 2+r_{c}\right)}{\sqrt{\left(X+U^{*}-b / 2+r_{c}\right)^{2}+\left(Y+V^{*}-b / 2+r_{c}\right)^{2}}} \\
& \operatorname{sen} \theta_{2}=\frac{\left(Y+V^{*}-b / 2+r_{c}\right)}{\sqrt{\left(X+U^{*}-b / 2+r_{c}\right)^{2}+\left(Y+V^{*}-b / 2+r_{c}\right)^{2}}} \\
& B_{3}=\sqrt{\operatorname{sen}^{2} \theta_{1}+\frac{\cos ^{2} \theta_{1}}{\cos ^{2}\left(\theta_{3}-\theta_{2}\right)}}
\end{aligned}
$$

\subsubsection{Condições de atrito}

É importante levar em conta o efeito do atrito na interface punção-chapa, principalmente, na distribuição de deformações. A diminuição do atrito nesta interface diminuirá a energia requerida para o processo de conformação, e permitirá que maior deformação seja levada a cabo com a mesma configuração de equipamento. A condição de atrito, neste estudo é caracterizada pela conhecida Lei de Coulomb:

$$
\tau=\mu p
$$


onde $\tau$ é a magnitude da tensão de atrito ao longo da interface, e $p$ é a pressão interfacial local. Considerando que a tensão de atrito atua na direção oposta ao deslocamento relativo entre a ferramenta e a chapa metálica, então a equação 5.90 pode ser expressa na forma:

$$
\tau=-\mu p \frac{\mathbf{u}_{r e l}}{\left|\mathbf{u}_{r e l}\right|}
$$

onde $\mathbf{u}_{r e l} /\left|\mathbf{u}_{r e l}\right|$ é o vetor unitário na direção do deslocamento relativo, $\mathbf{u}_{r e l}$. O coeficiente de atrito, é uma constante a ser determinada por testes onde as forças tangenciais e normais são medidas na superfície da matriz. 


\section{Implementação Computacional}

\subsection{Funcionamento do Programa}

Desenvolvido por Toh (1983) , O SHEET é um programa destinado a reproduzir importantes ensaios de conformação de chapas metálicas, que visam estabelecer os limites da conformabilidade de materiais diversos nas situações mais comuns do processo de estampagem (estiramento e estampagem profunda).

A partir de uma formulação de elementos finitos voltada para materiais rígido-plásticos, a mecânica da deformação das chapas metálicas pode ser estudada através da opção por um dos seguintes problemas:

Opção 1 - Estiramento de tira retangular (caso não axi-simétrico): consiste em produzir a deformação de uma chapa através da aplicação de uma força normal à sua superfície. A deformação é causada pelo deslocamento de um punção hemisférico perpendicularmente à superfície da chapa, de forma retangular, que é impedida de se deslocar nas extremidades. (figura 44 a)

Opção 2 - Estiramento de chapa circular (caso axi-simétrico): uma chapa de forma circular é impedida de se deslocar em toda a extensão do seu perímetro e submetida à deformação devida a força aplicada por um punção hemisférico que se desloca perpendicularmente (direção $z$ ) em relação à superfície da chapa (plano $x-y$ ).

Opção 3 - Estiramento de chapa semi-circular (caso não axi-simétrico): similar ao caso 2, porém a chapa circular agora tem recortes de forma circular (cut-off) onde 


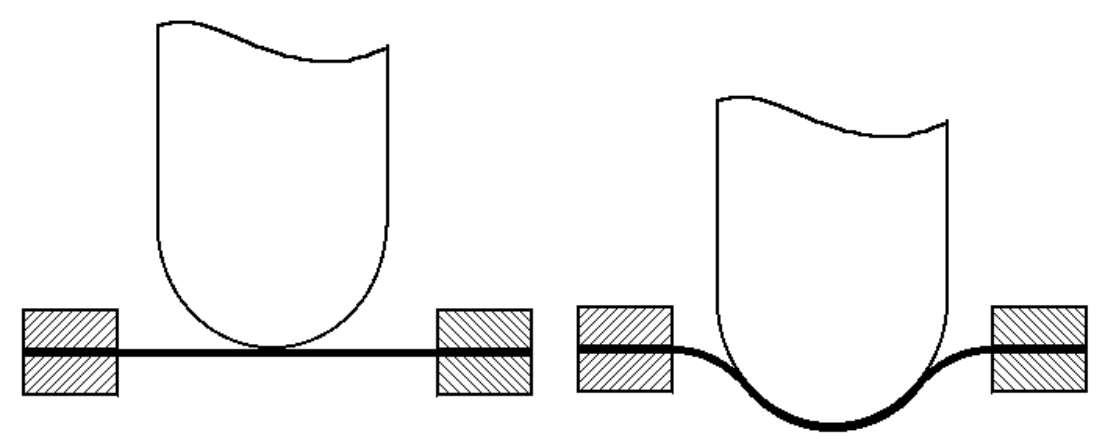

(a) Estiramento com punção hemisférico

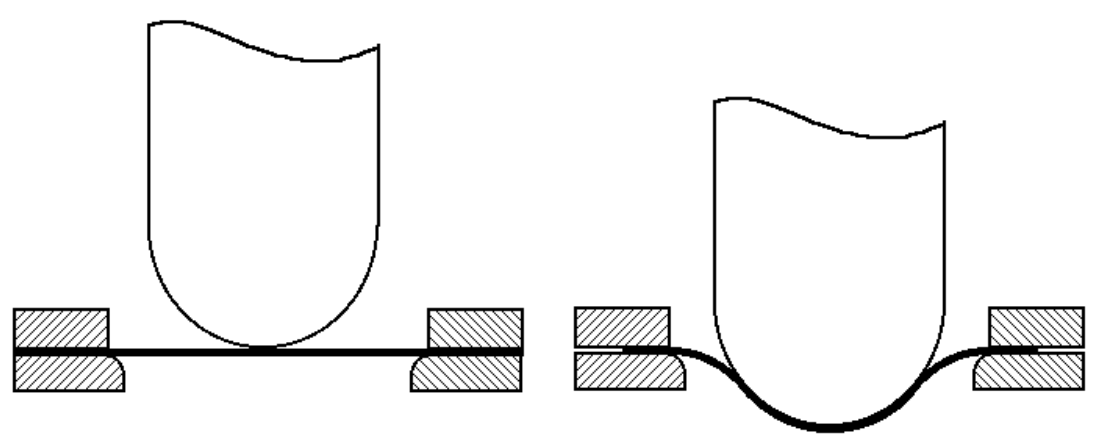

(b) Estampagem profunda com punção hemisférico

Figura 44: Vista esquemática do (a) estiramento e (b) estampagem profunda. (Toh (1983)).

não há restrição ao deslocamento.

Opção 4 - Estampagem profunda de copo cilíndrico (caso axi-simétrico): a chapa de forma circular tem restrição parcial ao deslocamento nas bordas, (flange) que não podem se deslocar na mesma direção que o punção hemisférico (z). A restrição imposta pelo prensa-chapas não impede que a chapa, na flange, se desloque no plano $x-y$ (figura $44 \mathrm{~b})$

Opção 5 - Estampagem profunda de copo quadrado (caso não axi-simétrico): similar ao caso 4, porém o punção agora é de seção quadrada e fundo plano. 


\subsection{Estrutura do Programa}

O código do programa é dividido em subrotinas, como listadas a seguir:

Principal: estabelece um vetor para a distribuição e manuseio dos dados de entrada. Abre o arquivo de entrada de dados e cria o arquivo de saída. Verifica o tipo de problema a ser estudado.

Stamp: lê os dados de entrada e aloca os dados no vetor de armazenamento de dados. Lê os parâmetros de material.

Datain: faz a leitura da localização relativa do nó na malha, coordenadas nodais, estimativas iniciais de deslocamento (deformação) e relações de conectividade.

Solve: estabelece os procedimentos de solução para cada um dos casos (ordena a seqüência de execução de cada uma das subrotinas envolvidas na solução do problema específico). Controla o avanço do punção em todas as etapas previstas para o processamento e controla a interferência (penetração) do punção na chapa através da redução do avanço do punção na etapa em que isto ocorrer.

Asembl: faz a montagem das matrizes de cada elemento segundo a equação 5.36 e 5.51 e monta o sistema de equações 5.55 .

Tranfm: transforma as coordenadas locais de cada nó do elemento em coordenadas globais. Ver seção 5.4

Strain: a partir da equação constitutiva rígido-plástica, faz o cálculo das tensões e deformações para cada elemento.

Boucon: incorpora as condições de contorno relativas às forças de tração e de geometria.

Geombc: faz a modificação da matriz de rigidez e do vetor de carga para as condições prescritas de deslocamento e grau de liberdade.

Cirblk: introduz as condições de contorno específicas para o caso da chapa circular . 
Bansol: faz a triangularização da matriz de rigidez global pelo método de eliminação de Gauss.

Thom: realiza os procedimentos interativos de solução do sistema de equações pelo método de Newton Raphson. O controle do processo é feito por um coeficiente de aceleração e a condição de convergência é verificada pela norma fracional. Ver seção 5.5. Os resultados de cada interação são impressos no arquivo de saída.

Raph: solução do sistema de equações para deslocamentos correspondentes aos vetores de carga.

Datout: imprime os resultados obtidos para os cálculos realizados para cada etapa (avanço do punção).

Force: calcula as forças nodais e carga total no punção.

Update: atualiza as geometrias e condições de contorno.

Squa: atualiza as condições de contorno para o punção de seção quadrada.

Deep: controla a movimentação dos nós localizados na flange no processo de estampagem profunda.

Dieras: atualiza as condições de contorno nos cantos da matriz.

Squadi: atualiza as condições de contorno para os cantos da matriz para o caso da estampagem profunda do copo de seção quadrada.

Uma facilidade oferecida pelo programa é geração dos dados relativos aos nós, automaticamente, através da informação dos dados nodais adequados.

\subsection{Validação do Programa SHEET}

A validação do programa SHEET para a estampagem profunda foi feita comparando os resultados obtidos com os apresentados por Toh (1983). Como alguns parâmetros 


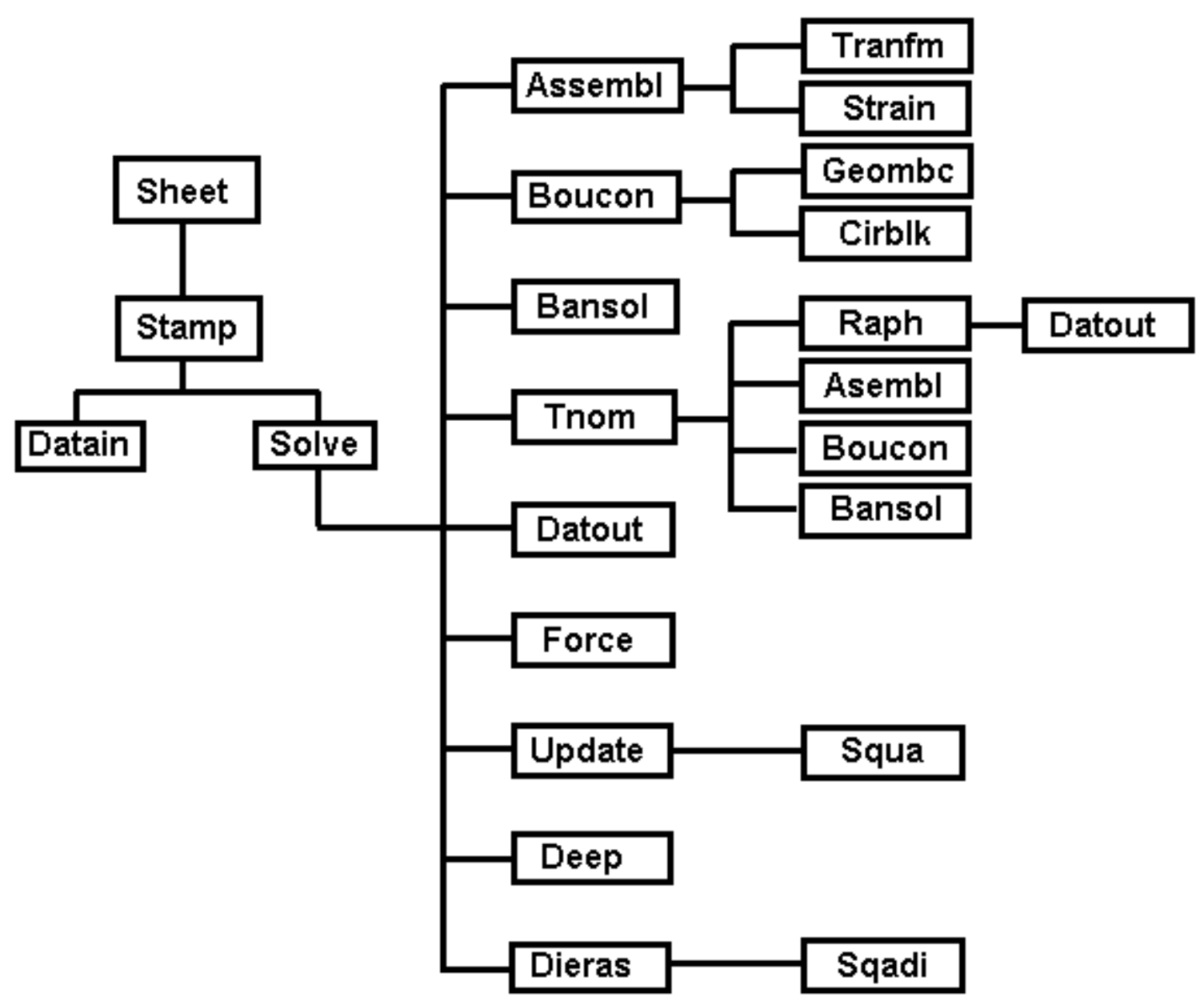

Figura 45: Fluxograma do programa SHEET (Toh (1983)).

(malha usada, avanço do punção, deslocamentos iniciais, tolerância de contato) usados por Toh (1983) não estão disponíveis, sendo necessário portanto a verificação destes na influência dos resultados obtidos.

\subsubsection{Estampagem profunda com punção hemisférico}

Os parametros fornecidos por Toh (1983) foram:

material cobre macio

Valor $\boldsymbol{R}^{1} 1,0$

\footnotetext{
${ }^{1}$ Coeficiente de anisotropia normal
} 
lei constitutiva

$$
\begin{array}{ll}
\text { para } \varepsilon<0,36: & \bar{\sigma}=\left(0,08208+0,42257 \bar{\varepsilon}^{0,504}\right) \mathrm{GPa} \\
\operatorname{para} \varepsilon>0,36: & \bar{\sigma}=\left(0,08208+0,37089 \bar{\varepsilon}^{0,375}\right) \mathrm{GPa}
\end{array}
$$

espessura da chapa $0,889 \mathrm{~mm}$

raio do blank $55,88 \mathrm{~mm}$

diametro da abertura da matriz $53,92 \mathrm{~mm}$

raio do punção $25,4 \mathrm{~mm}$

raio da matriz $12,7 \mathrm{~mm}$

força do prensa-chapas $4900 \mathrm{~N}$

coeficientes de atrito $\mu_{p}=\mu_{d}=0,04$ para o punção e matriz, respectivamente

O programa usa para a modelagem numérica apenas uma parte do blank, uma seção de $10^{\circ}$ (figura 41). Para esta seção foi usada a malha na figura 46, com 81 elementos e 55 nós. Com esta malha obtiveram-se os seguintes resultados para a distribuição da deformação nas espessuras para várias razões $r_{b} / R_{b}$, onde $R_{b}$ é o raio inicial do blank e $r_{b}$ o raio final da chapa, e força no punção, figuras 47 e 48, respectivamente. Para se chegar aos presentes resultados foram necessários vários testes com diversas malhas, e para cada malha sendo simulada com uma variação dos parâmetros não fornecidos por Toh (1983), como o avanço do punção. A malha da figura 46 foi a que obteve os resultados mais próximos aos de Toh (1983).

\subsubsection{Estampagem profunda com punção quadrado}

Os parâmetros fornecidos por Toh (1983) foram (ver figura 42):

material aço acalmado com alumínio 


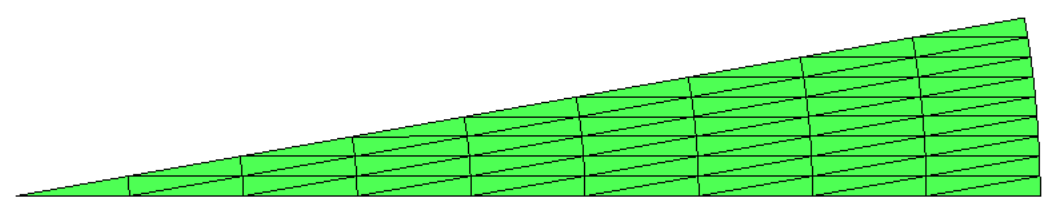

Figura 46: Malha usada na estampagem profunda com punção hemisférico.

Valor $\boldsymbol{R} 1,6$

lei constitutiva $\bar{\sigma}=739 \bar{\varepsilon}^{0,228} \mathrm{MPa}$

espessura da chapa $0,86 \mathrm{~mm}$

dimensão do punção, $\boldsymbol{a} 40 \mathrm{~mm}$ x $40 \mathrm{~mm}$

dimensão da matriz, $\boldsymbol{b} 42,5 \mathrm{~mm}$ x $42,5 \mathrm{~mm}$

raio do punção, $\boldsymbol{r}_{\boldsymbol{p}} 5 \mathrm{~mm}$

raio da matriz, $\boldsymbol{r}_{\boldsymbol{d}} 5 \mathrm{~mm}$

raio de canto, $\boldsymbol{r}_{\boldsymbol{c}} 3,2 \mathrm{~mm}$

dimensão do blank $110 \mathrm{~mm}$ x $110 \mathrm{~mm}$

coeficientes de atrito $\mu_{p}=0,2$ e $\mu_{d}=0,04$ para o punção e matriz, respectivamente força do prensa-chapas $4900 \mathrm{~N}$

Para a modelagem, o programa necessita de apenas 1/8 do blank, como mostrado na figura 43, e foi discretizada com 76 elementos e 53 nós (figura 49), conforme Toh 


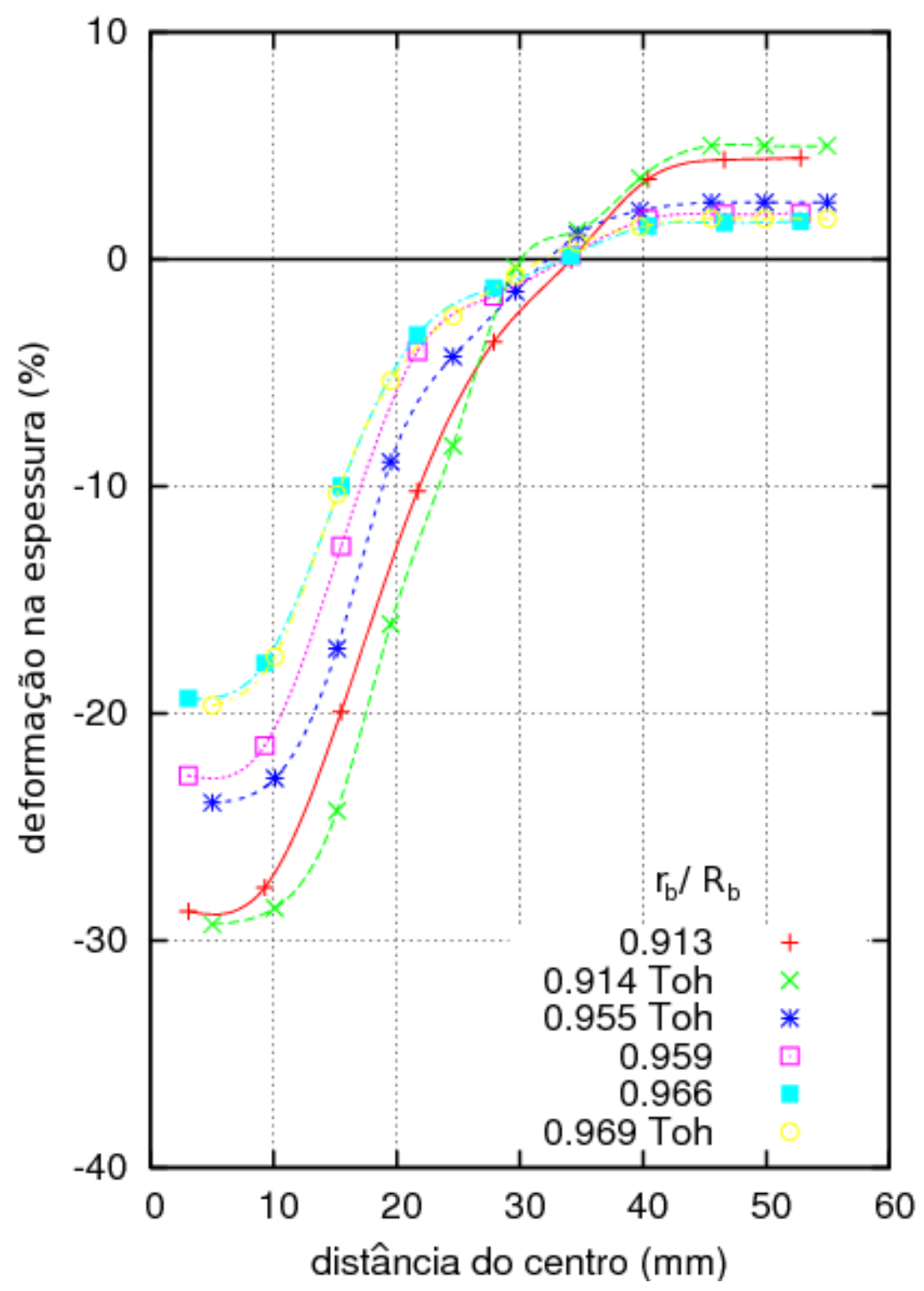

Figura 47: Comparação entre os resultados obtidos por Toh (1983) e a presente dissertação, para a distribuição da deformação na espessura para vários $r_{b} / R_{b}$.

(1983). Os resultados obtidos foram comparados aos de Toh (1983) (figura 50) a uma profundidade de 20,2 mm, podendo se observar um discrepância no valor em um elemento. Isso ocorre devido ao fato da malha não se identica à de Toh. Malhas com diferenças de milímetros entre os nós produziram resultados diferentes (por exemplo, figura 51), sendo necessário uma variação da localização dos nós para se obter a malha da figura 49, a qual mais se aproximou dos resultados obtidos por Toh. A malha deformada na profundidade de 20,2 mm pode ser vista na figura 55. Outros gráficos comparando os resultados da presente dissertação com os obtidos por Toh (1983) podem ser vistos nas figuras 52, 53 e 54. 


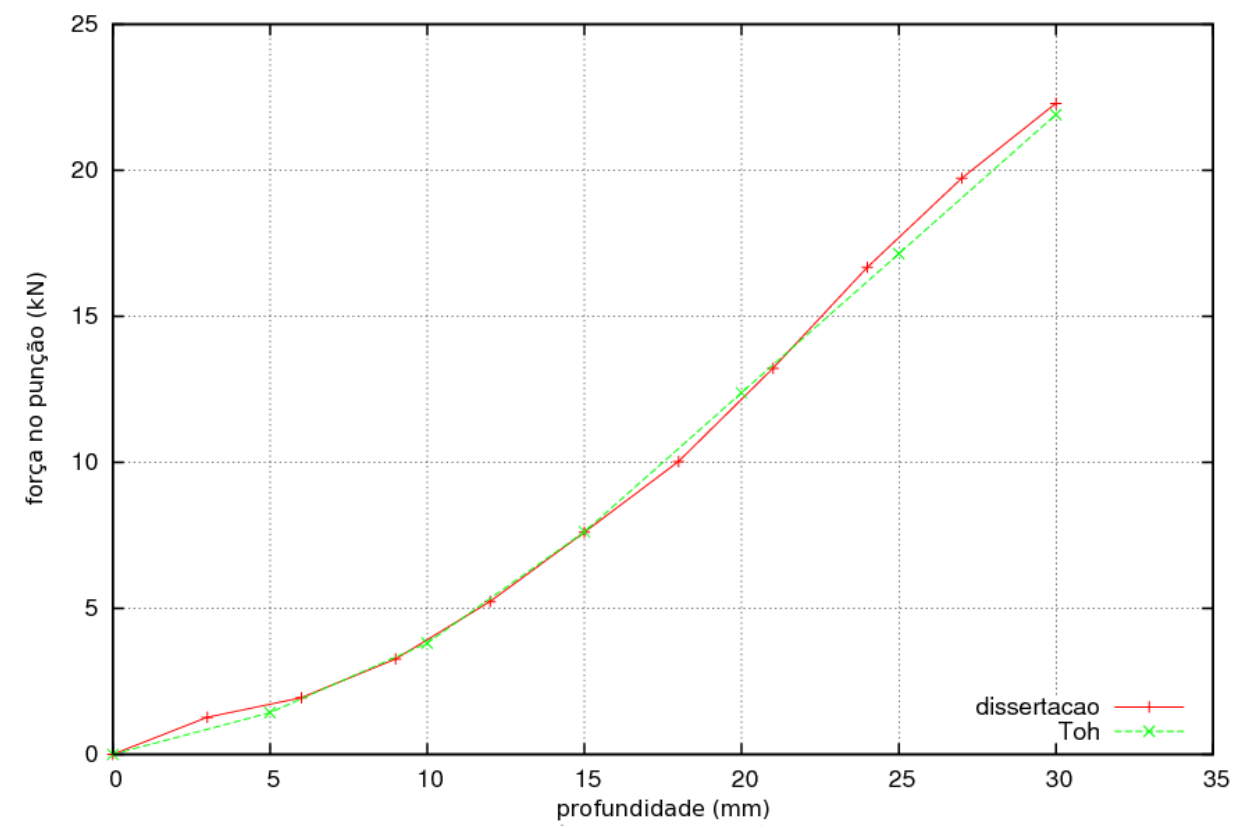

Figura 48: Comparação entre os resultados obtidos por Toh (1983) e a presente dissertação, para a força no punção.

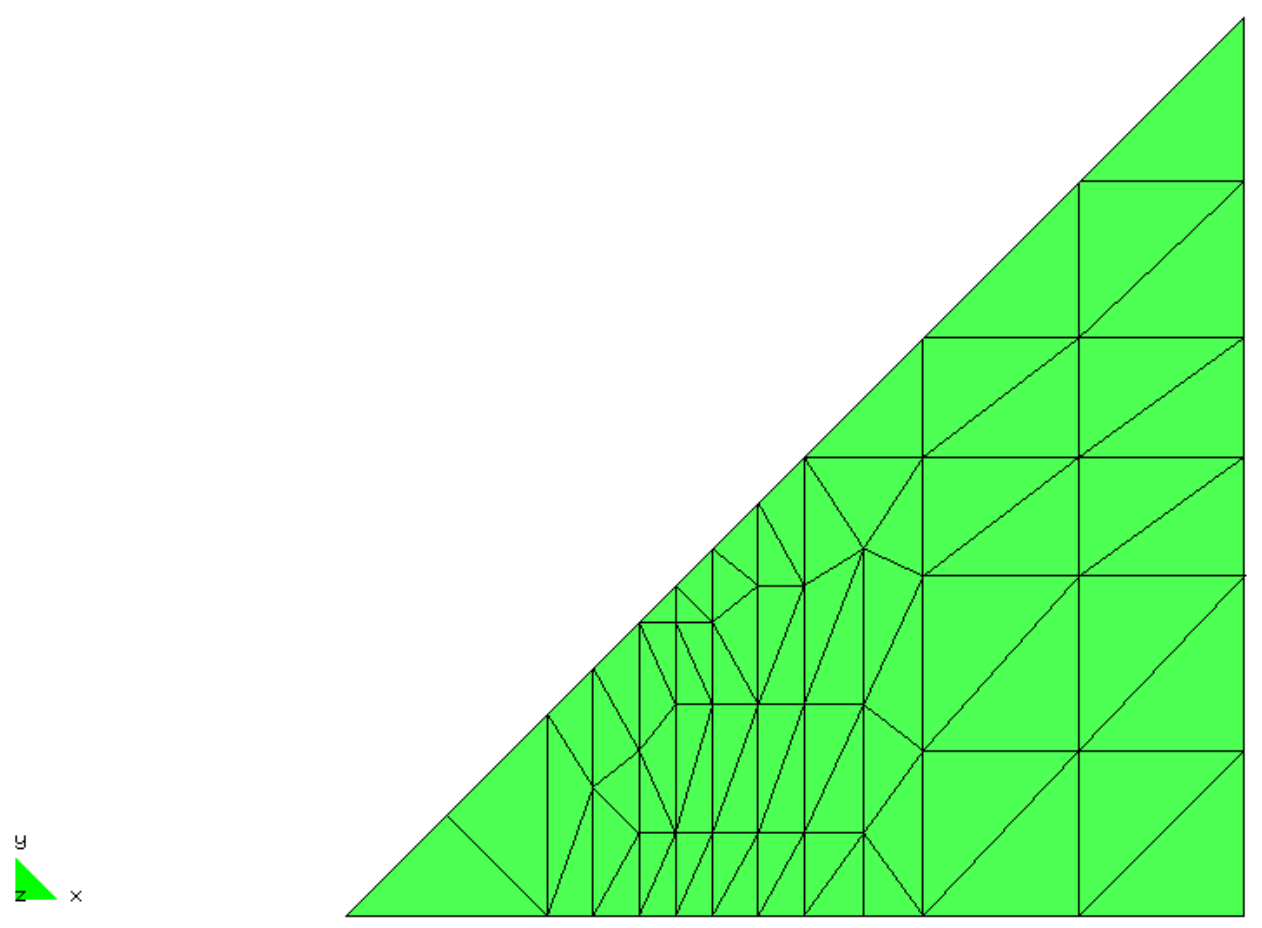

Figura 49: Malha usada na modelagem. 


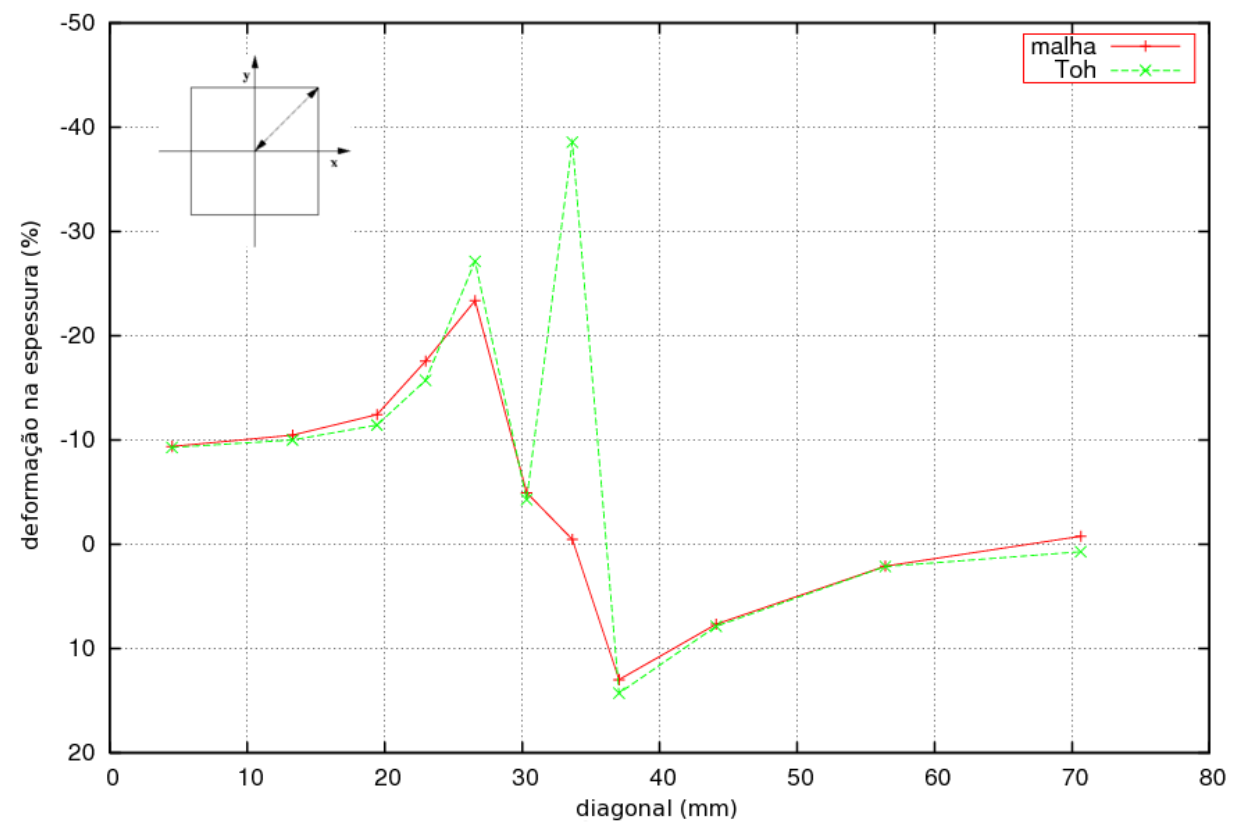

Figura 50: Deformação na espessura na diagonal, comparação dos resultado obtidos com os de Toh (1983).

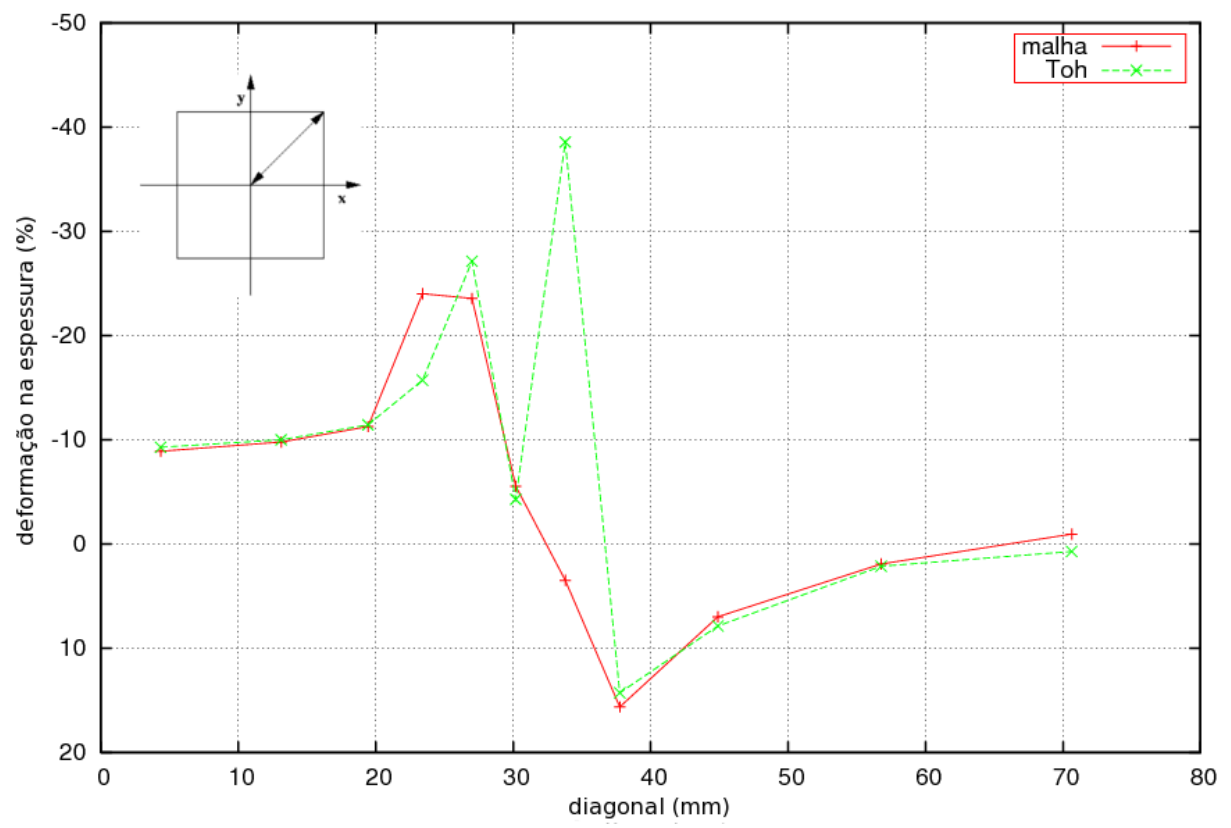

Figura 51: Deformação na espessura em uma malha não otimizada. 


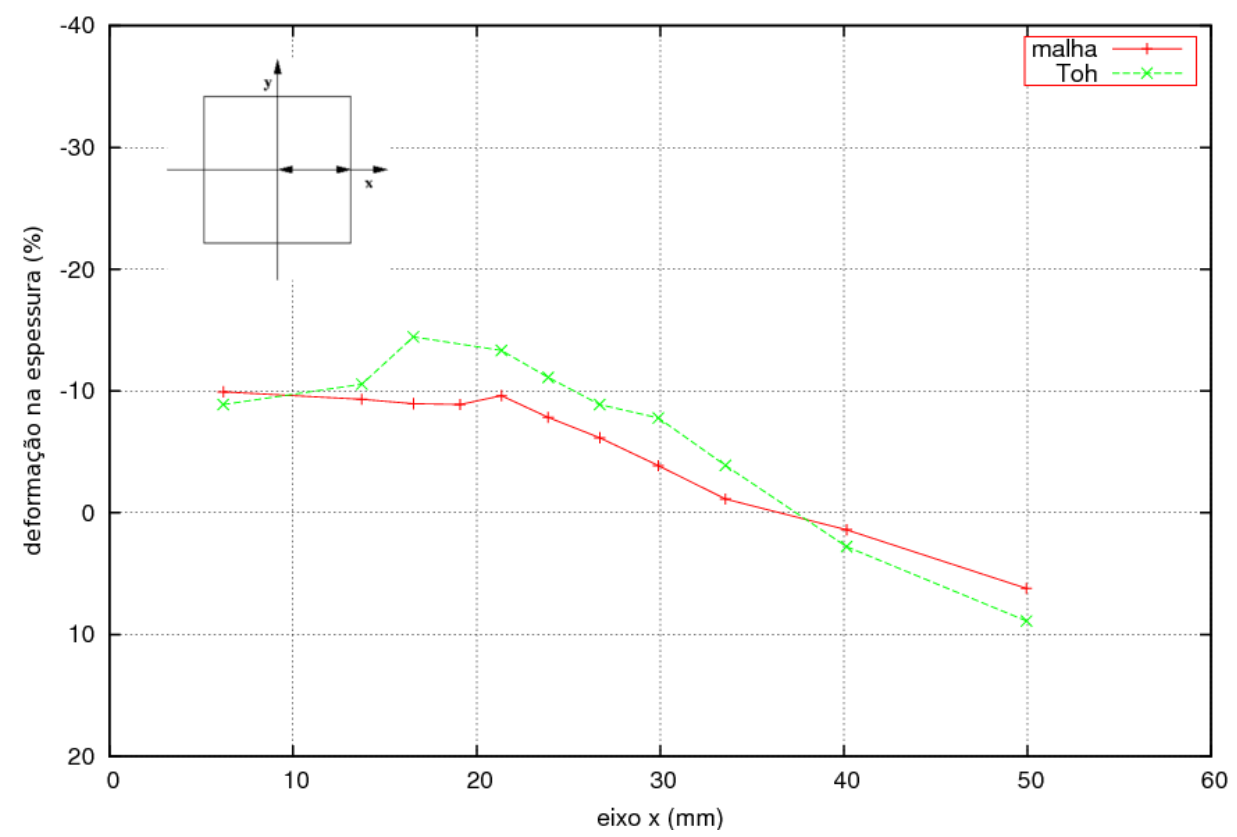

Figura 52: Deformação na espessura no eixo $x$, comparação dos resultados obtidos com os de Toh (1983).

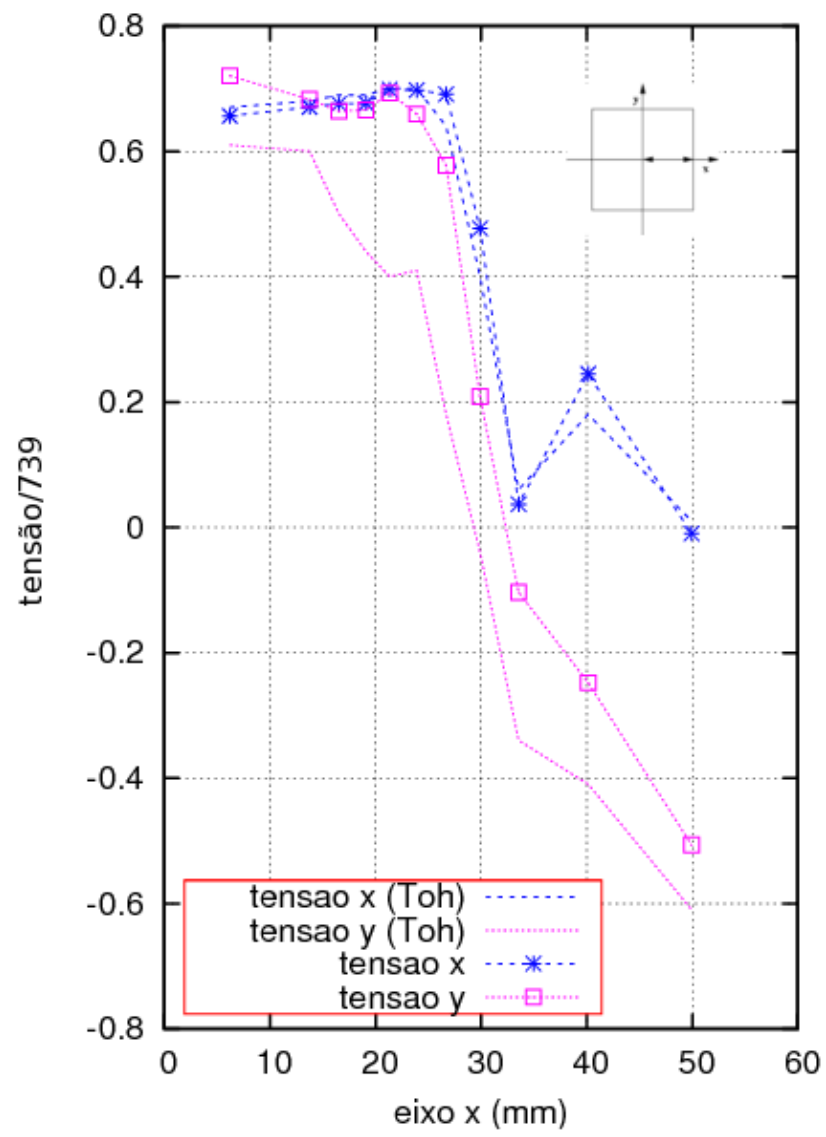

Figura 53: Distribuição da tensão no eixo $x$ (dividido pela constante $K$, equação 3.87), comparação dos resultados obtidos com os de Toh (1983). 


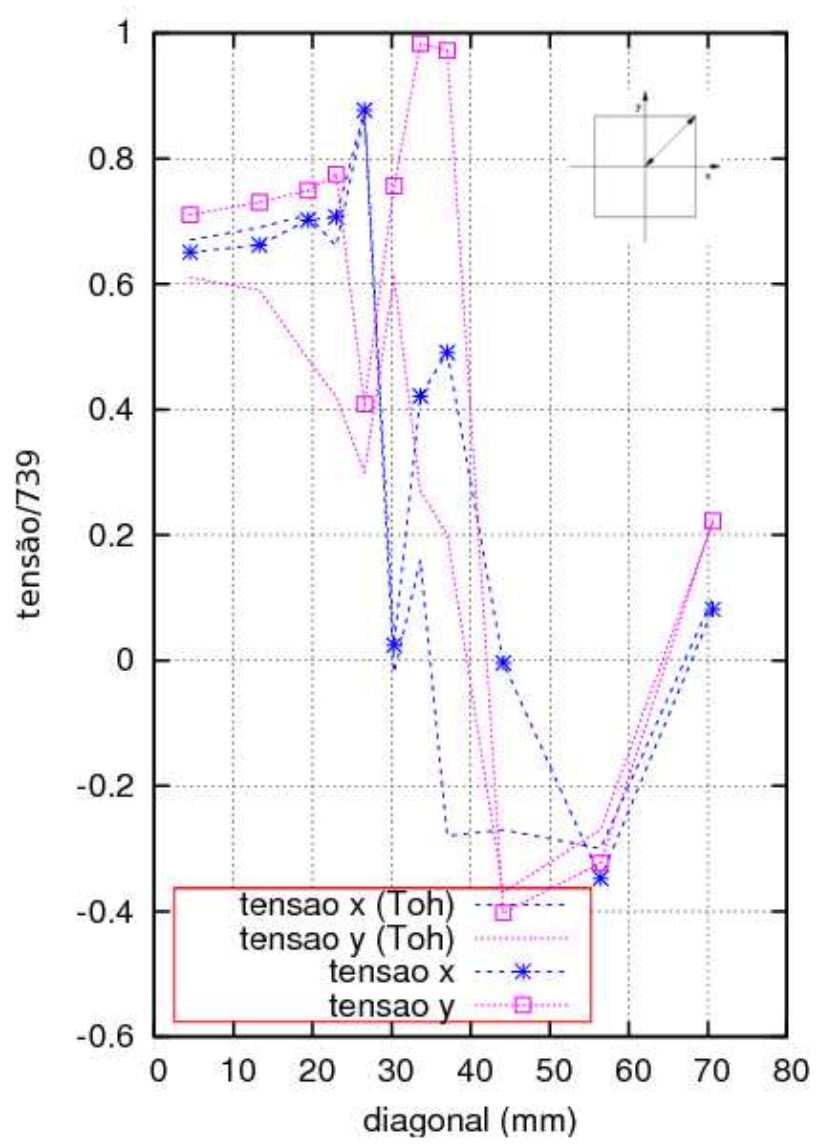

Figura 54: Distribuição da tensão na diagonal (dividido pela constante $K$, equação 3.87), comparação dos resultados obtidos com os de Toh (1983).

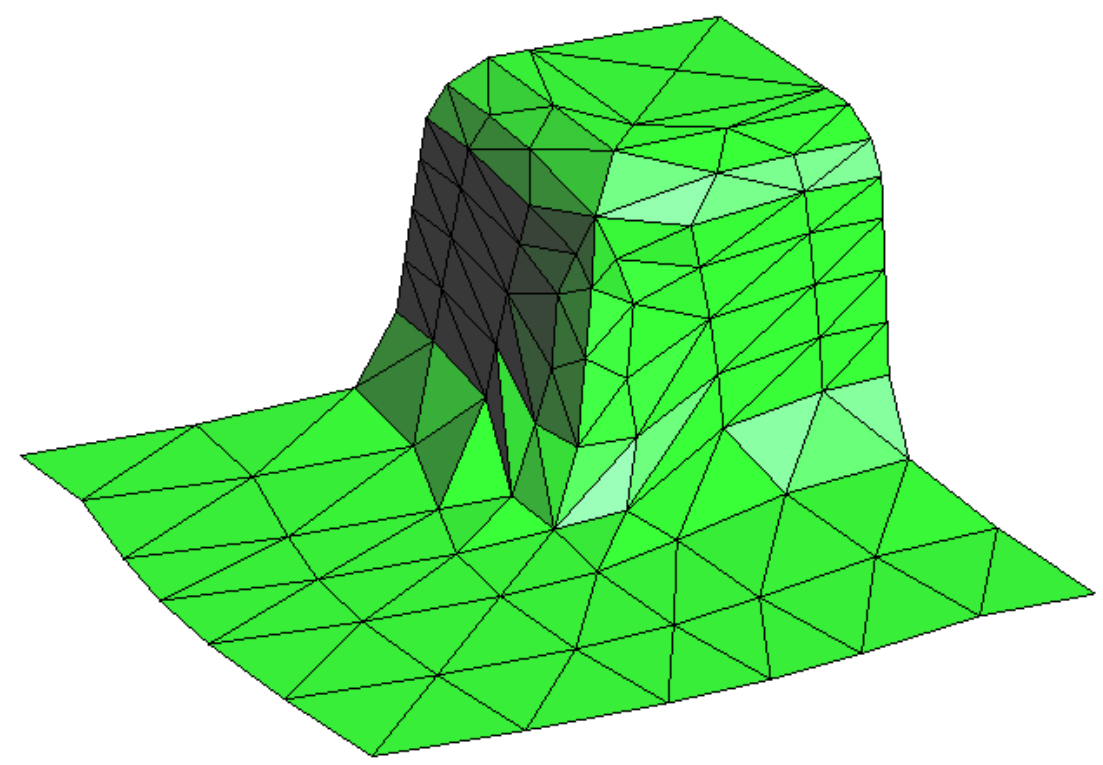

Figura 55: Malha deformada na profundidade final de 20,2 $\mathrm{mm}$. 


\section{Resultados e Discussões}

Para a inclusão do critério de escoamento de Hill (1979) no programa SHEET as sub-rotinas ASEMBL e STRAIN (ver seção 6.2) foram modificadas. As equações 5.52 e 5.53 foram incluidas na sub-rotina ASEMBL e a equação 3.69 é incluida na sub-rotina STRAIN. As sub-rotinas modificadas estão listadas no Anexo A.

Resultados numéricos obtidos com o programa SHEET modificado são apresentados. O primeiro problema trata do estiramento de um blank circular de aço inoxidável AK STEEL ${ }^{\circledR} 409$ HP-10 sendo os resultados obtidos comparados com dados experimentais de Comstock Jr et al. (2001). No segundo problema e terceiro problema a estampagem profunda com punção quadrado é modelado. No segundo, a estampagem é feita usando o alumínio e, no terceiro, é usado um aço baixo carbono (mild steel). Os resultados obtidos com valores de $M$ (ver seção 3.5) variando de 1,6 a 2,0 são comparados com experimentos realizados por Danckert (1995).

Para demonstar que a o critério de Hill (1979) retorna para Hill (1950) quando $M=2,0$, o problema da seção 6.3.2 é modelado no programa modificado com $M=2,0$ e os demais parâmetros idênticos à seção 6.3.2. O resultado para distribuição da deformação na diagonal pode ser visto na figura 56.

\subsection{Estiramanto com punção hemisférico}

Os parametros fornecidos por Comstock Jr et al. (2001) foram 


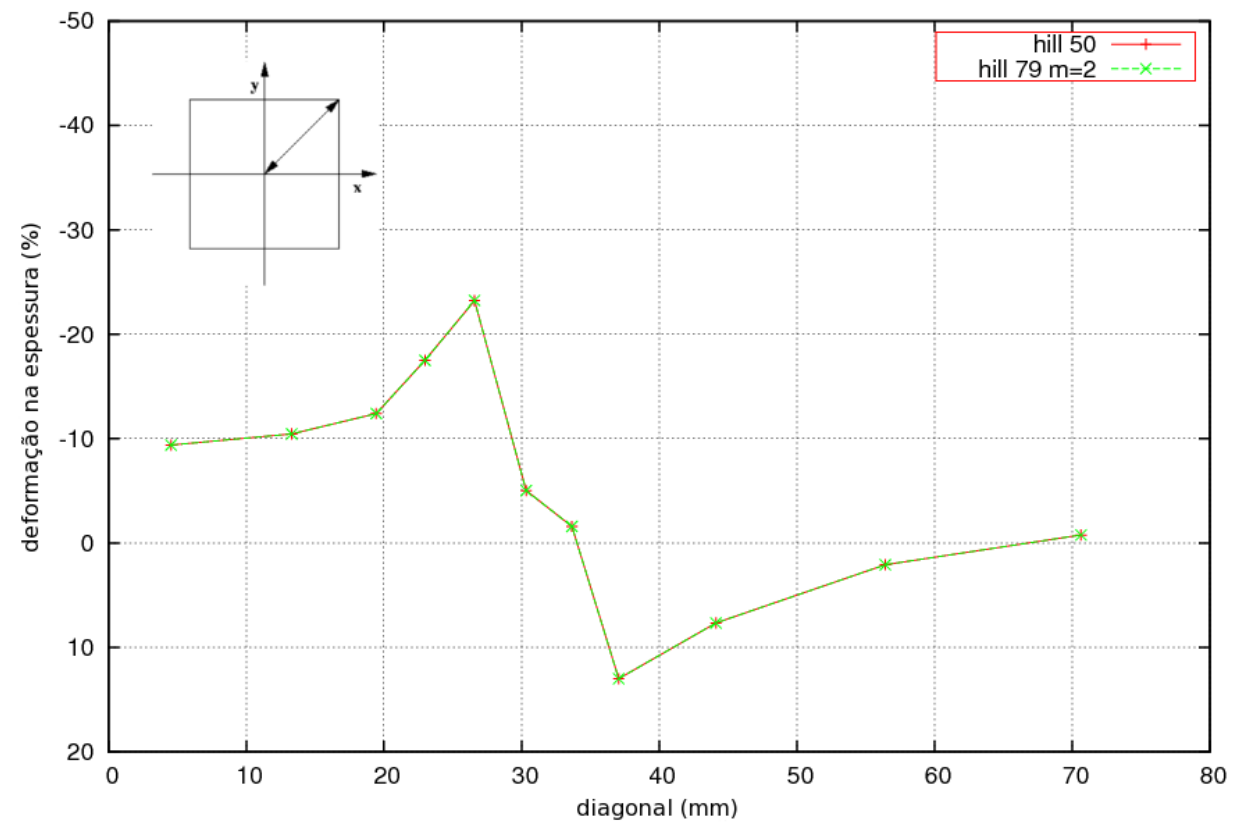

Figura 56: Comparação entre os resultados obtidos pelo programa SHEET original e o SHEET modificado.

Valor $\boldsymbol{R} 1,49$

lei constitutiva $\bar{\sigma}=711 \bar{\varepsilon}^{0,199} \mathrm{MPa}$

espessura da chapa $1,17 \mathrm{~mm}$

raio do blank $13,8 \mathrm{~mm}$

diâmetro da abertura da matriz $13,8 \mathrm{~mm}$

coeficientes de atrito $\mu_{p}=0,13$ para o punção

Para a modelagem foi usada uma seção de $10^{\circ}$ com a seguinte malha, figura 57 , com 17 elementos e 19 nós. O problema foi modelado com o parâmetro de Hill $M=2,0$ e $M=1,9$. Os resultados para as deformações principais podem ser vistos nos gráficos da figura 58. Os resultados obtidos mostram uma melhora significativa nas curvas quando $M=1,9$. Esta melhora se deve ao fato de que a diminuição dos valores de $M$ leva a um alongangamento da superfície de escoamento na direção do carregamento biaxial (figuras 59 e 60 ), isto leva a necessidade de maiores tensões principais $\left(\sigma_{1}\right.$ e $\left.\sigma_{2}\right)$ para o 

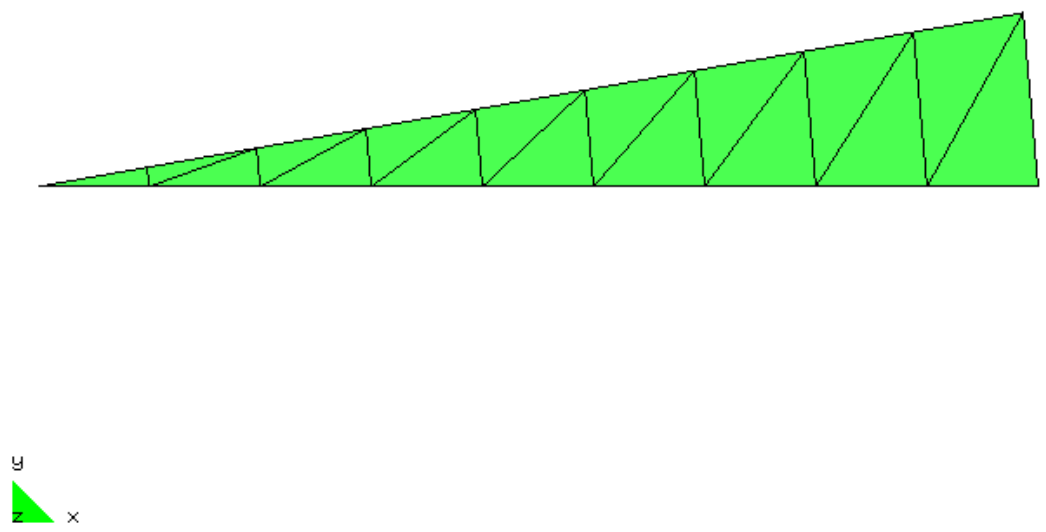

Figura 57: Malha usada no estiramento de blank circular

escoamento plástico, então uma pressão maior é necessária para que a mesma configuração de deformação seja alcançada, aumentando a pressão de contato entre o punção e a chapa, o que leva a um efeito semelhante ao aumento do coeficiente de atrito entre a chapa e o punção, observado por Wang (1984), no caso há o aumento da força normal que leva ao aumento da força de atrito. 

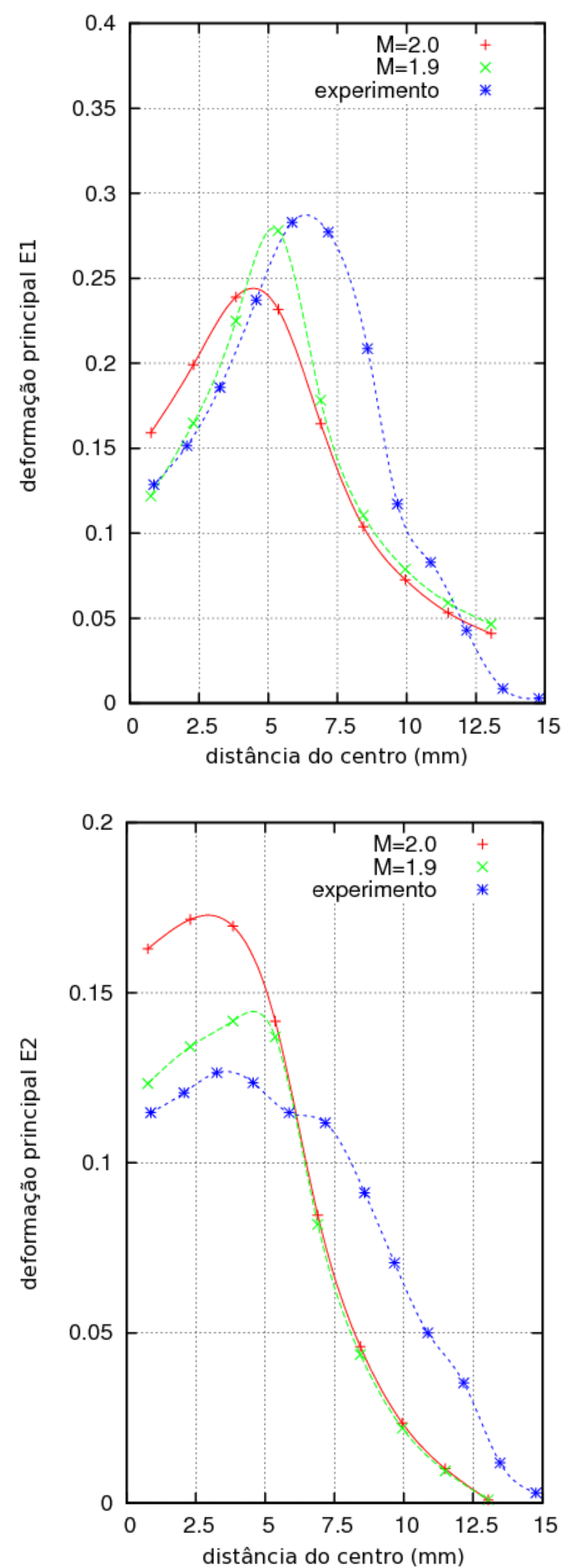

Figura 58: Comparação entre os resultados obtidos numericamente e os resultados experimentais 


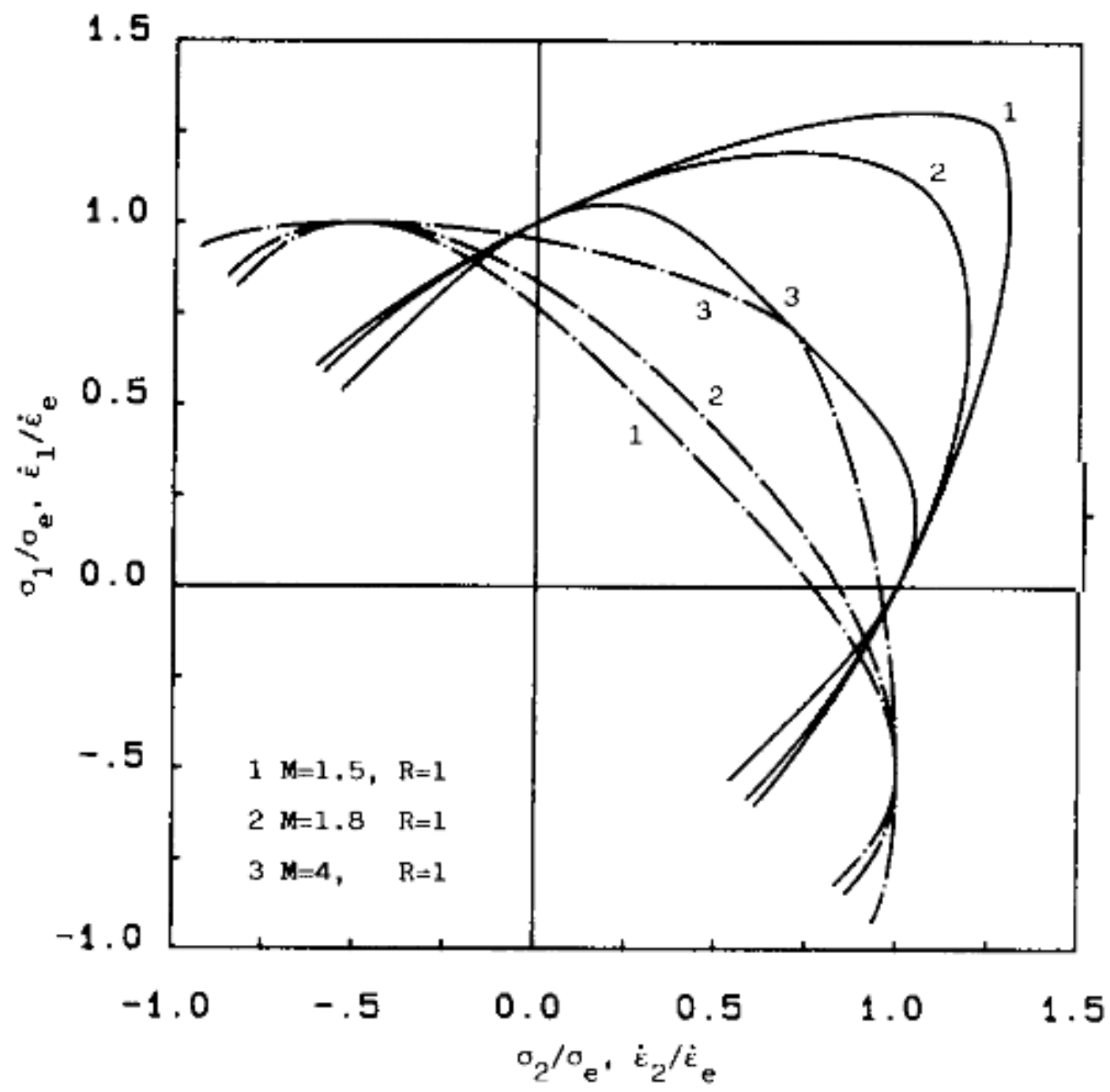

Figura 59: Superfícies de escoamento para a tensão plana (linhas contínuas) e superfície da taxa de deformação associada (linhas descontínuas) (Lian et al. (1989)). 


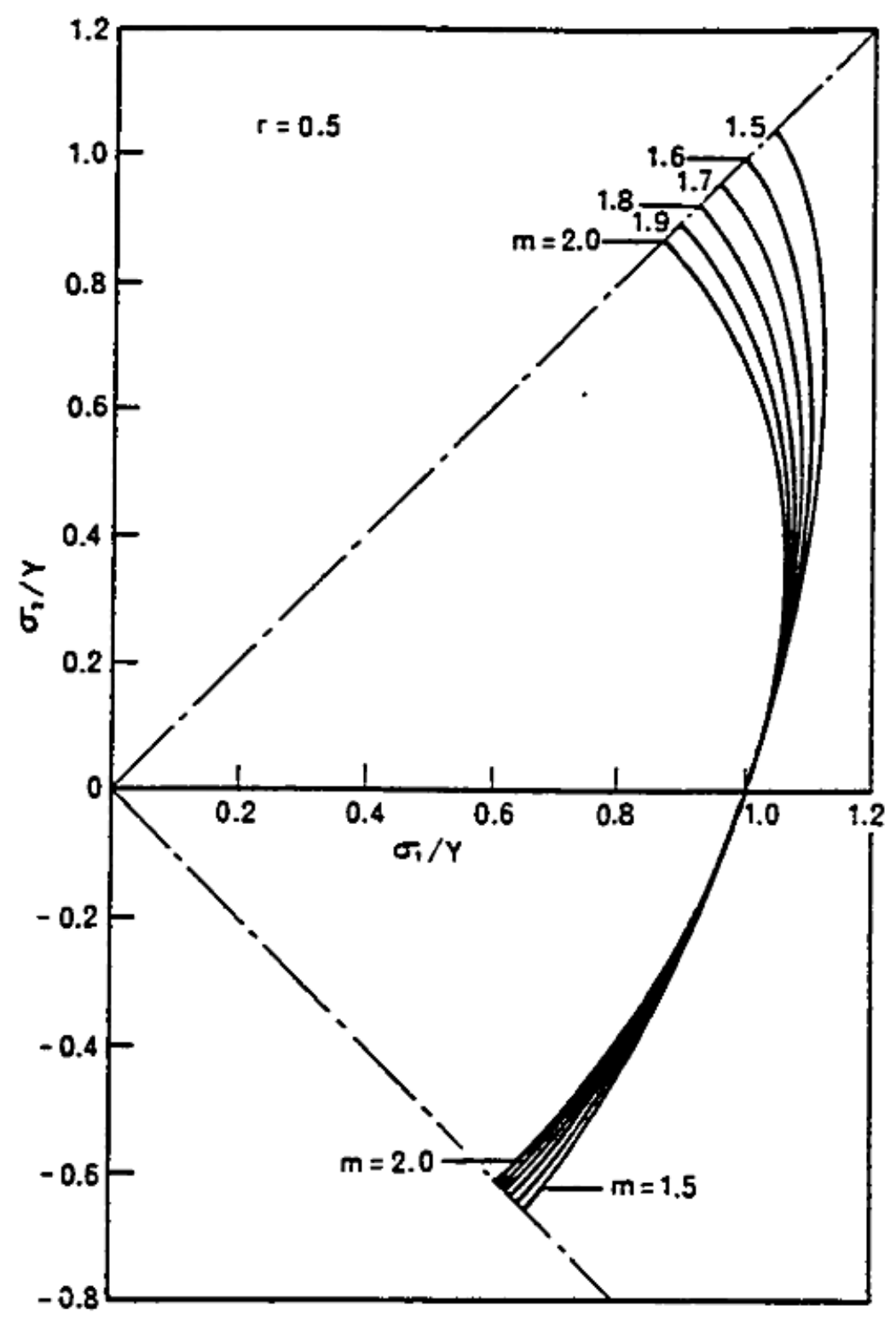

Figura 60: Superfície de escoamento para tensão plana, $r=R=0,5$ e $m$ é o mesmo que $M$ (Parmar e Mellor (1978)). 


\subsection{Estampagem profunda com punção quadrado}

\subsubsection{Alumínio}

Os parâmetros fornecidos por Danckert (1995) foram

material Alumínio

Valor $\boldsymbol{R} 0,64$

lei constitutiva $\bar{\sigma}=576,79(0,01658 \bar{\varepsilon})^{0,3593} \mathrm{MPa}$

espessura da chapa $0,81 \mathrm{~mm}$

dimensão do punção, $\boldsymbol{a} 70 \mathrm{~mm} \times 70 \mathrm{~mm}$

dimensão da matriz, $\boldsymbol{b} 74 \mathrm{~mm} \times 74 \mathrm{~mm}$

raio do punção, $\boldsymbol{r}_{\boldsymbol{p}} 8 \mathrm{~mm}$

raio da matriz, $\boldsymbol{r}_{\boldsymbol{d}} 5 \mathrm{~mm}$

raio de canto do punção, $\boldsymbol{r}_{\boldsymbol{c p}} 10 \mathrm{~mm}$

raio de canto da matriz, $\boldsymbol{r}_{\boldsymbol{c d}} 12 \mathrm{~mm}$

dimensão do blank $150 \mathrm{~mm}$ x $150 \mathrm{~mm}$

coeficientes de atrito $\mu_{p}=0,162$ e $\mu_{d}=0,162$ para o punção e matriz respectivamente

força do prensa-chapas $19620 \mathrm{~N}$

Para a modelagem foi usado $1 / 8$ do blank como mostrado na figura 61 , onde pode-se ver a malha usada que possui 196 elementos e 120 nós. O problema foi modelado com a força no prensa chapas igual a zero, pois o problema não convergia para força maior que zero. Foram obtidos resultados para valores de $M$ de 2,0 a 1,6. Os resultados foram comparados com resultados experimentais fornecido pela conferência NUMISHEET 93 
(Machinouchi (1993)) e resultados computacionais de Chung et al. (2000), figura 62. O resultado computacional de Chung et al. (2000) usado é para força no prensa-chapas igual a zero. As figuras 63 e 64 mostram a distribuição da deformação na espessura em 1/4 da peça final na profundidade de $15 \mathrm{~mm}$ e em 1/8 do blank não deformada, respectivamente, para $M=2,0$ e $M=1,6$. Observando-se a figura 62 ve-se que diminuindo-se o $M$ temse uma pequena diminuição dos valores da deformação na região do punção e nenhuma mudança significativa para as demais regiões. 


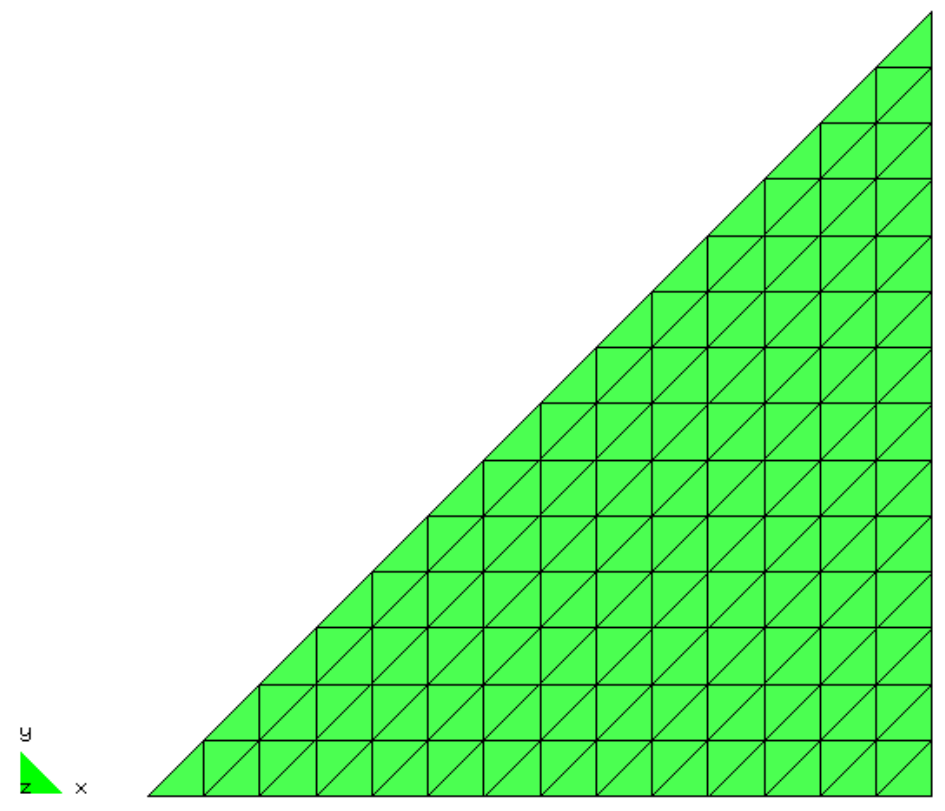

Figura 61: Malha usada na estampagem profunda

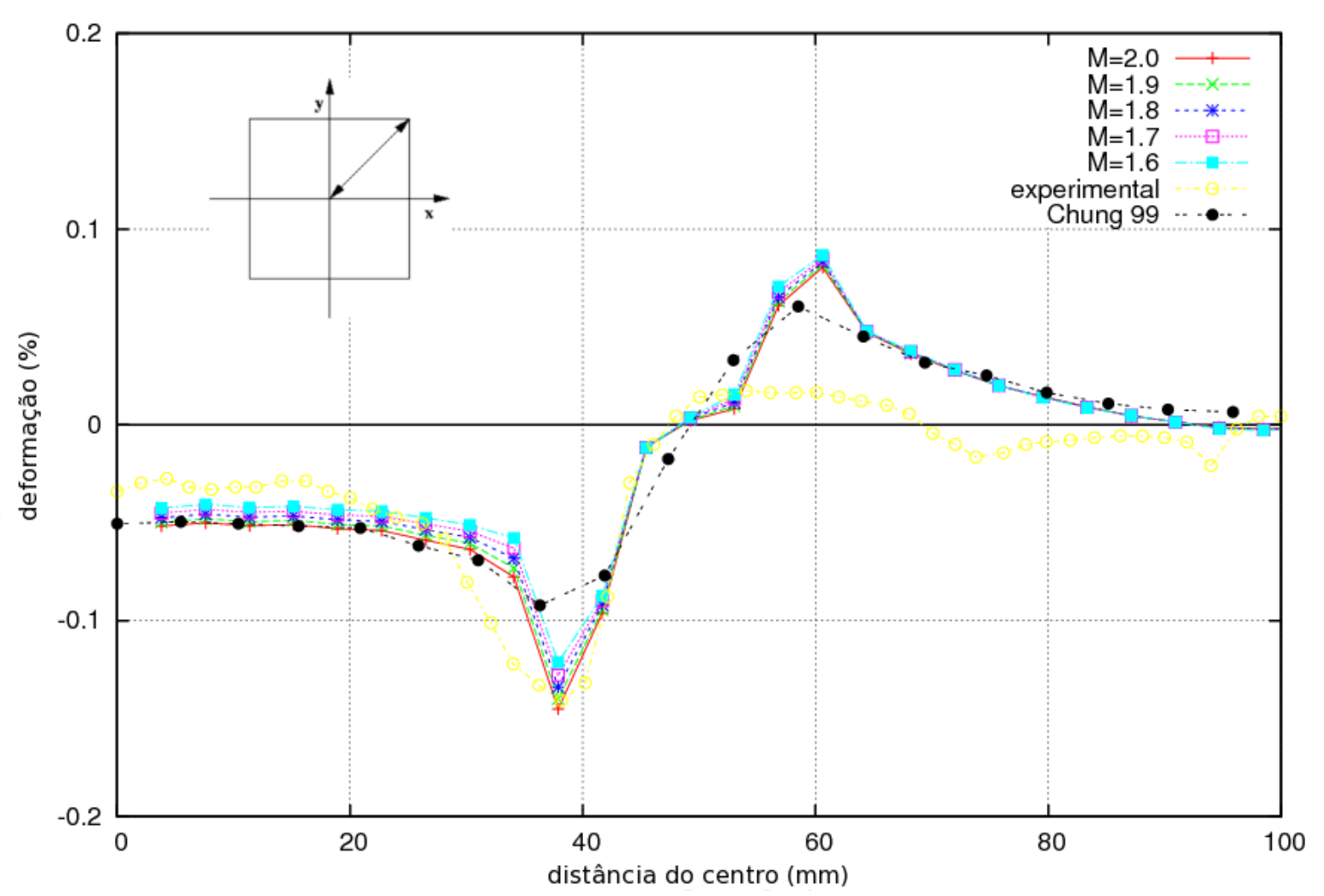

Figura 62: Deformação na espessura na diagonal 


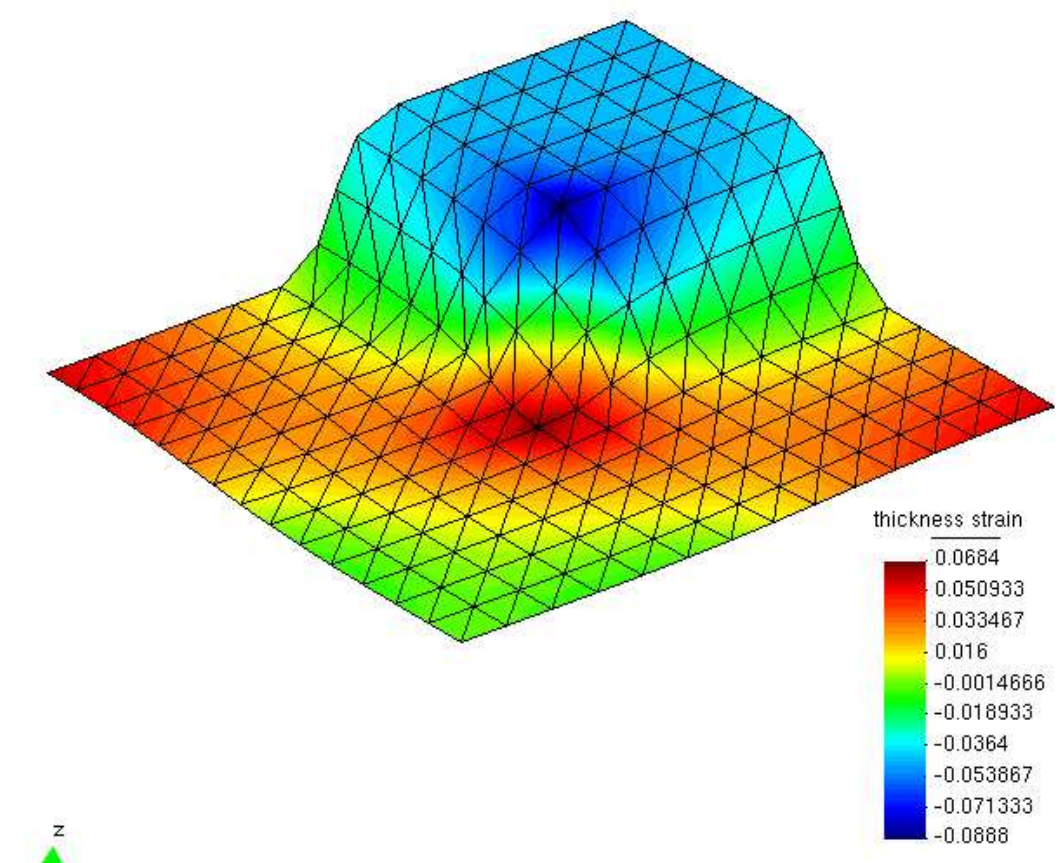

Contour Fill of Deslocamentos, |Deslocamentos|. Deformation (x1): Deslocamentos of Analise, step 1

a)

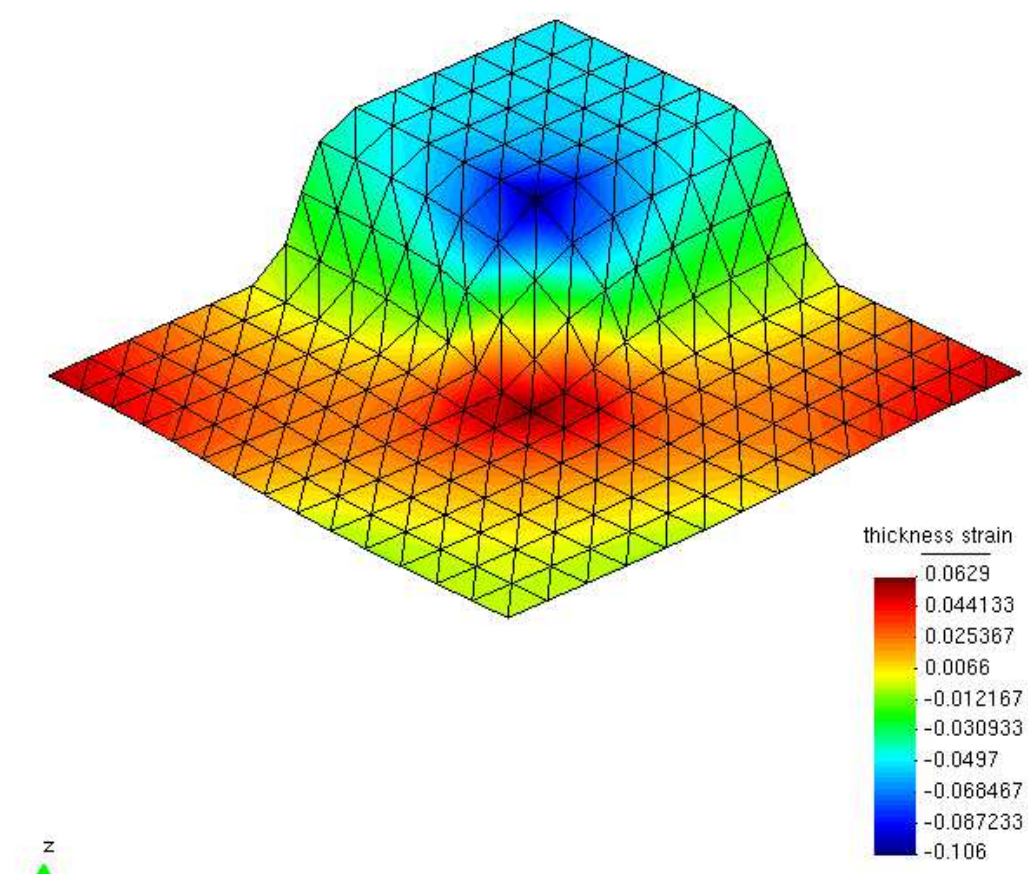

Contour Fill of Deslocamentos, |Deslocamentos|. Deformation (x1): Deslocamentos of Analise, step 1

b)

Figura 63: Distribuição da deformação na espessura na peça final: a) $M=1,6$ e b) $M=$ 2,0 


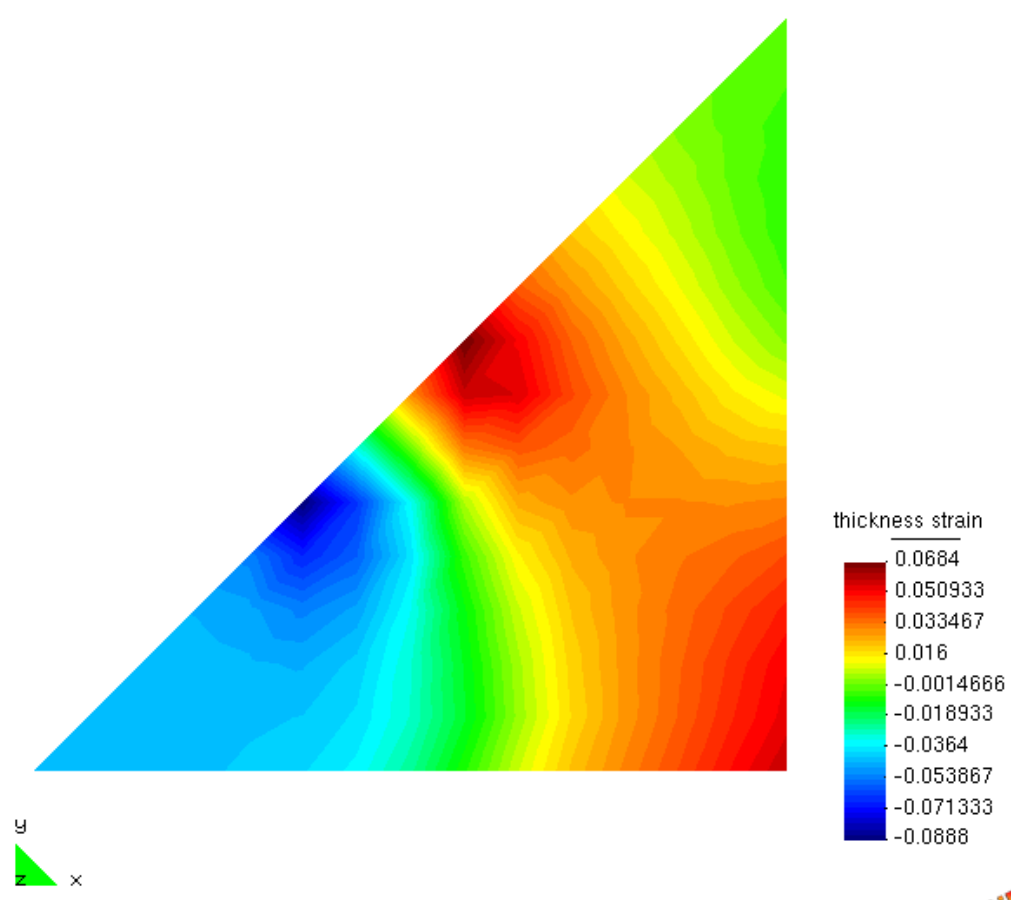

Contour Fill of Deformacoes.

a)

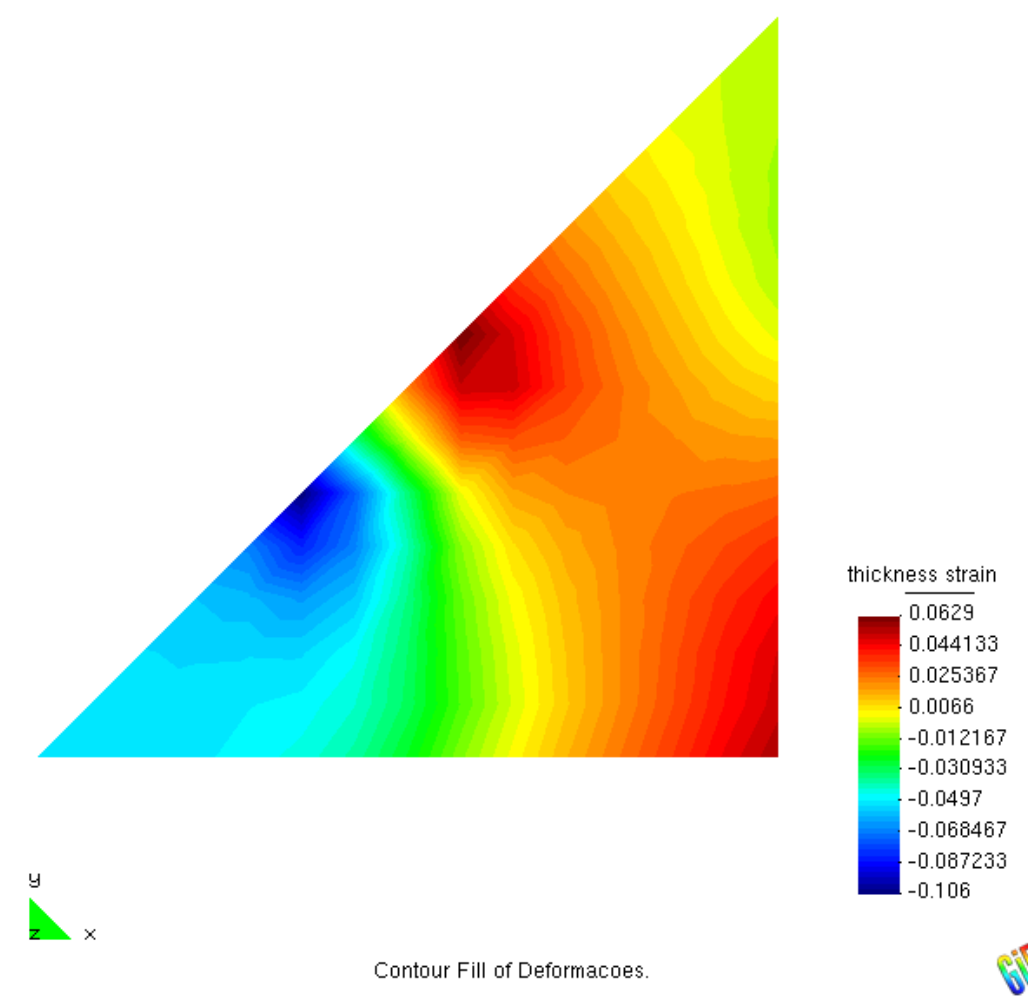

b)

Figura 64: Distribuição da deformação na espessura: a) $M=1,6$ e b) $M=2,0$ 


\subsubsection{Aço baixo carbono}

Os parâmetros fornecidos por Danckert (1995) foram

material aço carbono

Valor $\boldsymbol{R} 1,77$

lei constitutiva $\bar{\sigma}=565,32(0,007117 \bar{\varepsilon})^{0,2589} \mathrm{MPa}$

espessura da chapa $0,78 \mathrm{~mm}$

dimensão do punção, $\boldsymbol{a} 70 \mathrm{~mm} \times 70 \mathrm{~mm}$

dimensão da matriz, $\boldsymbol{b} 74 \mathrm{~mm} \times 74 \mathrm{~mm}$

raio do punção, $\boldsymbol{r}_{\boldsymbol{p}} 8 \mathrm{~mm}$

raio da matriz, $\boldsymbol{r}_{\boldsymbol{d}} 5 \mathrm{~mm}$

raio de canto do punção, $\boldsymbol{r}_{\boldsymbol{c} \boldsymbol{p}} 10 \mathrm{~mm}$

raio de canto da matriz, $\boldsymbol{r}_{\boldsymbol{c d}} 12 \mathrm{~mm}$

dimensão do blank 150 mm x 150 mm

coeficientes de atrito $\mu_{p}=0,144$ e $\mu_{d}=0,144$ para o punção e matriz respectivamente força do prensa-chapas $19620 \mathrm{~N}$

Para a modelagem foi usado o mesmo blank e malha do problema anterior, figura 61. O problema também foi modelado com a força no prensa chapas igual a zero, pois o problema não convergia para força maior que zero. Foram obtidos resultados para valores de $M$ de 2,0 a 1,65. Os resultados foram comparados com resultados experimentais obtidos por Danckert (1995) para profundidades de 15mm (figura 66) e profundidade de $40 \mathrm{~mm}$ (figuras 67 e 68). As figuras 69 e 72 mostram a distribuição da deformação na espessura em 1/8 do blank para profundidades de $15 \mathrm{~mm}$ e $40 \mathrm{~mm}$, respectivamente. As 
figuras 70 e 73 mostram 1/4 da peça, com a distribuição de deformação na espessura, para profundidades de $15 \mathrm{~mm}$ e $40 \mathrm{~mm}$. As figuras 71 e 74 mostram a formação de orelhas na estampagem profunda. As tabelas 1 e 2 mostram os deslocamentos na diagonal e na direção $X$, respectivamente, para os valores de $M$ usados na modelagem e compara com os valores experimentais de Danckert (1995). Como no problema anterior, vê-se pela figura 66 e mais nitidamente na figura 67 a redução da deformação na região do punção. Este fato também é explicado pelo alongamento da superfície de tensão de escoamento na direção do carregamento biaxial conforme se diminue o valor de $M$. Vê-se pela figura 59 que para uma diminuição do valor de $M$ há um alongamento da curva de tensão de escoamento e uma diminuição das deformações principais na direção do carregamaneto biaxial. Como na região plana do punção há um carregamento próximo do biaxial, as deformações nesta região diminuem. Nas demais regiões o carregamento é semelhante à tração uniaxial (região da parede) e de compressão uniaxial (região da aba), onde a mudança no parâmetro $M$ não tem muita influência.

\begin{tabular}{lcccccc}
\multicolumn{7}{c}{ Deslocamento na diagonal "DD" $(\mathrm{mm})$} \\
\hline & $M=2$ & $M=1,9$ & $M=1,85$ & $M=1,75$ & $M=1,65$ & experimental \\
\hline profundidade $15 \mathrm{~mm}$ & $-3,07$ & $-3,08$ & $-3,1$ & $-3,11$ & $-3,17$ & $-3,13$ \\
profundidade 40 mm & $-13,66$ & $-13,82$ & $-13,86$ & $-14,03$ & $-14,14$ & $-15,78$ \\
\hline
\end{tabular}

Tabela 1: Tabela com deslocamentos em DD. Valores experimentais por Danckert (1995).

\begin{tabular}{lcccccc}
\multicolumn{7}{c}{ Deslocamento na direção $X$} \\
\hline & $M=2$ & $M=1,9$ & $M=1,85$ & $M=1,75$ & $M=1,65$ & experimental \\
\hline profundidade 15 mm & $-5,36$ & $-5,4$ & $-5,4$ & $-5,47$ & $-5,51$ & $-6,16$ \\
profundidade 40 mm & $-24,6$ & $-24,8$ & $-24,9$ & $-25,2$ & $-25,5$ & $-28,64$ \\
\hline
\end{tabular}

Tabela 2: Tabela com deslocamentos em DX, Valores experimentais por Danckert (1995). 


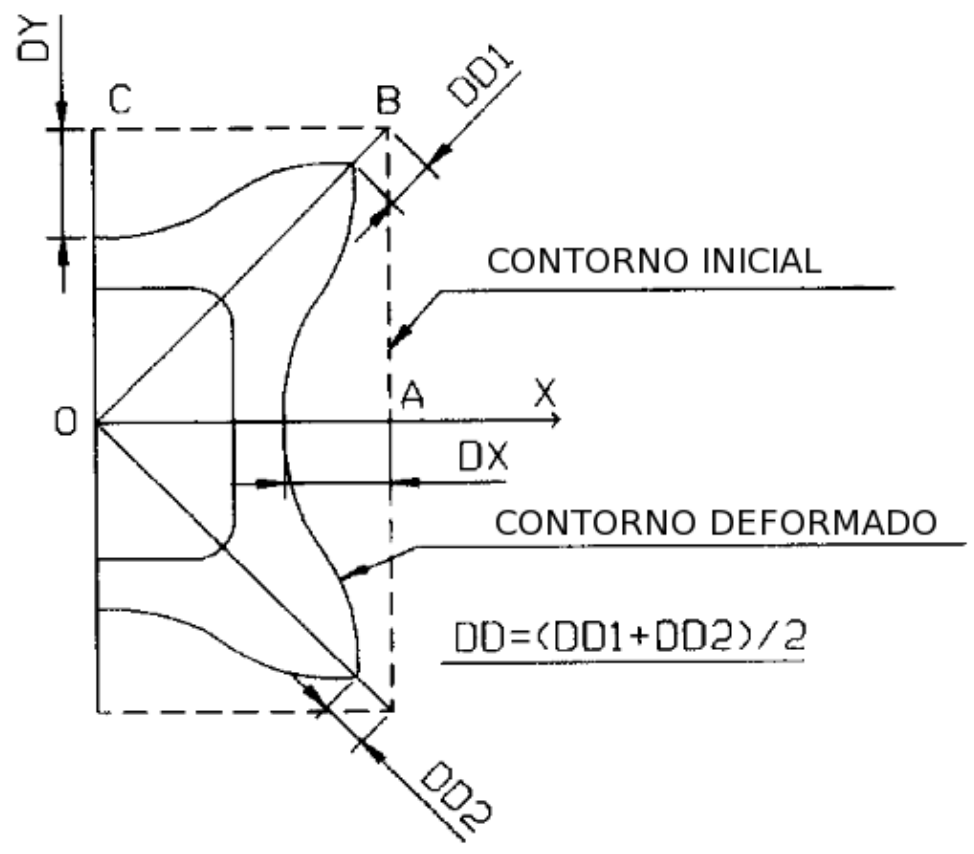

Figura 65: Parâmetros mensuradas Danckert (1995).

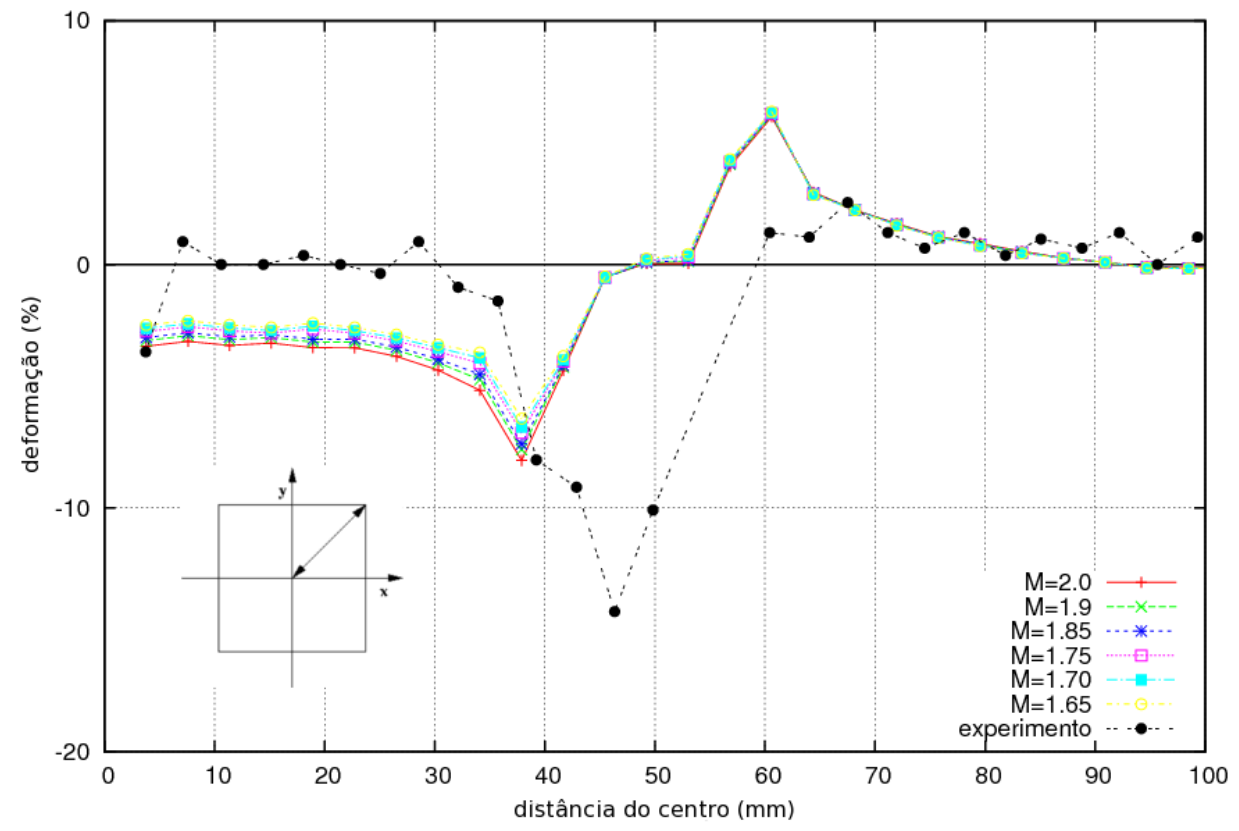

Figura 66: Deformação na espessura na diagonal. 


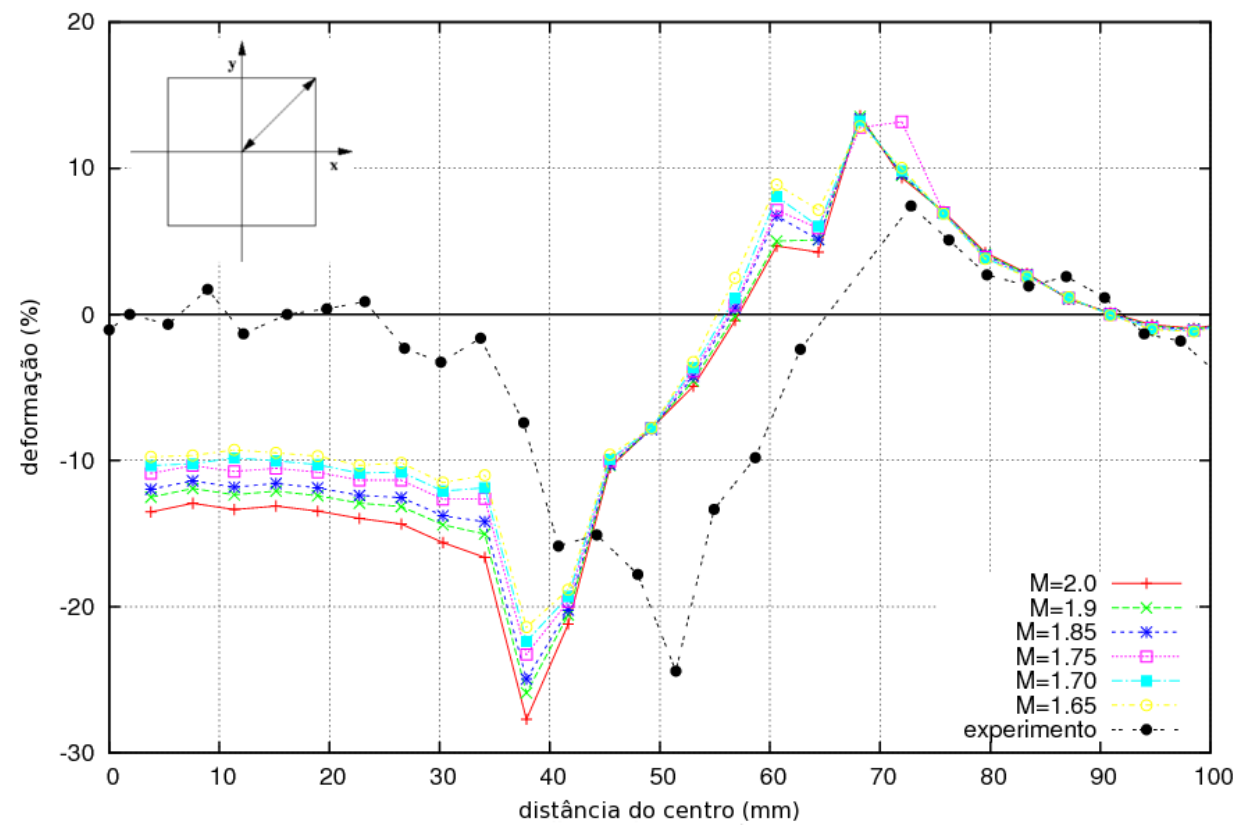

Figura 67: Deformação na espessura na diagonal.

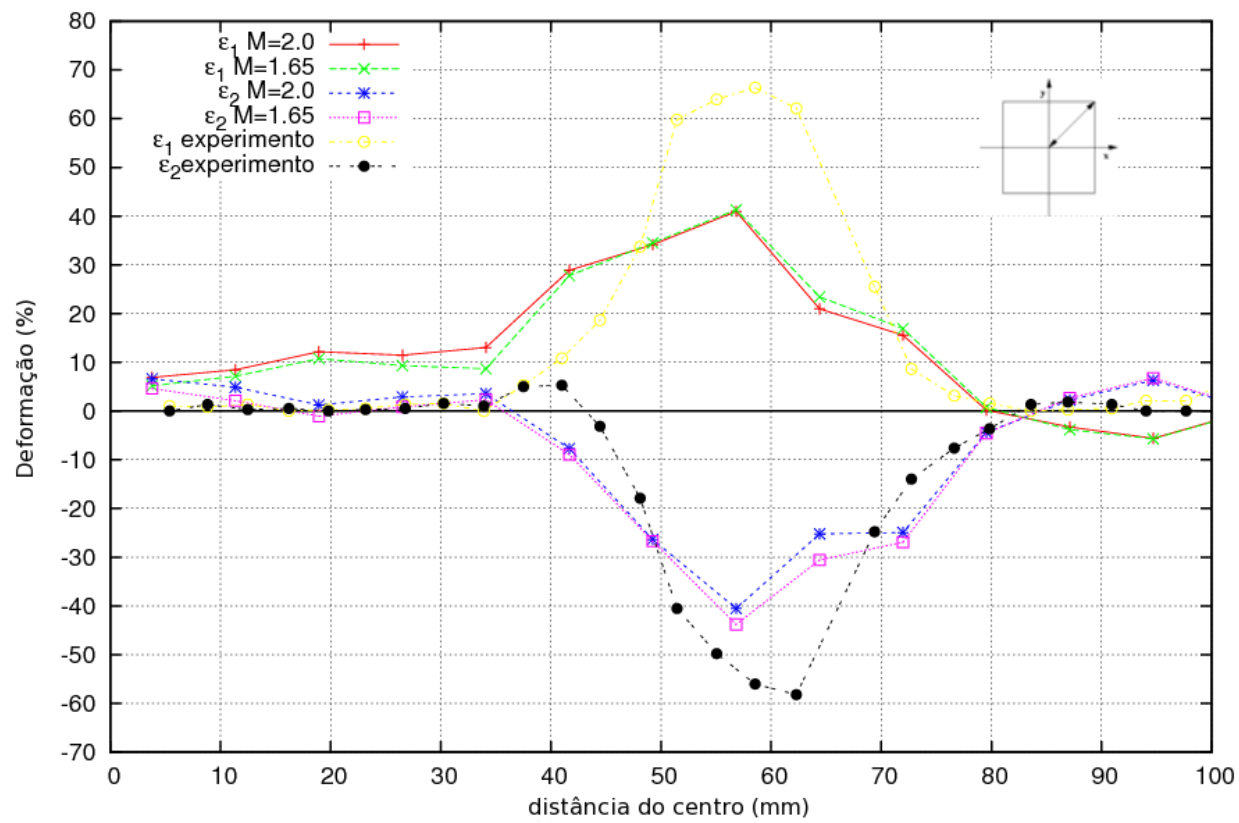

Figura 68: Deformações principais na diagonal. 


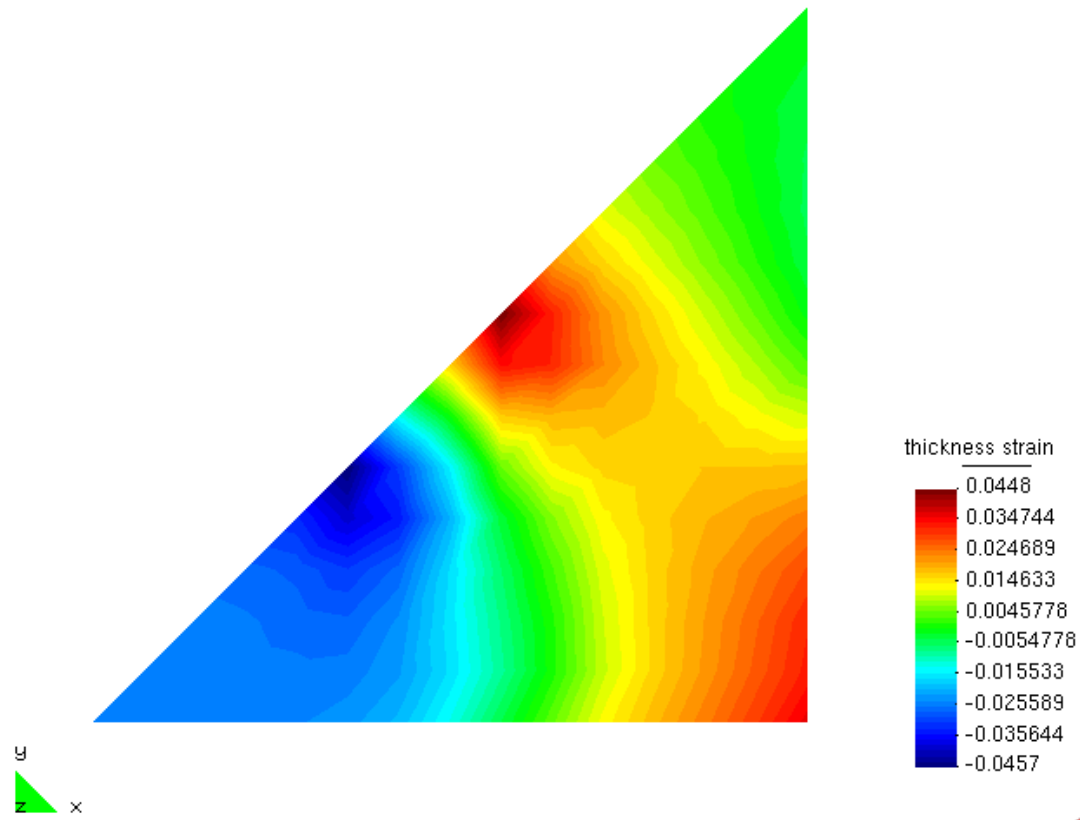

Contour Fill of Deformacoes.

a)

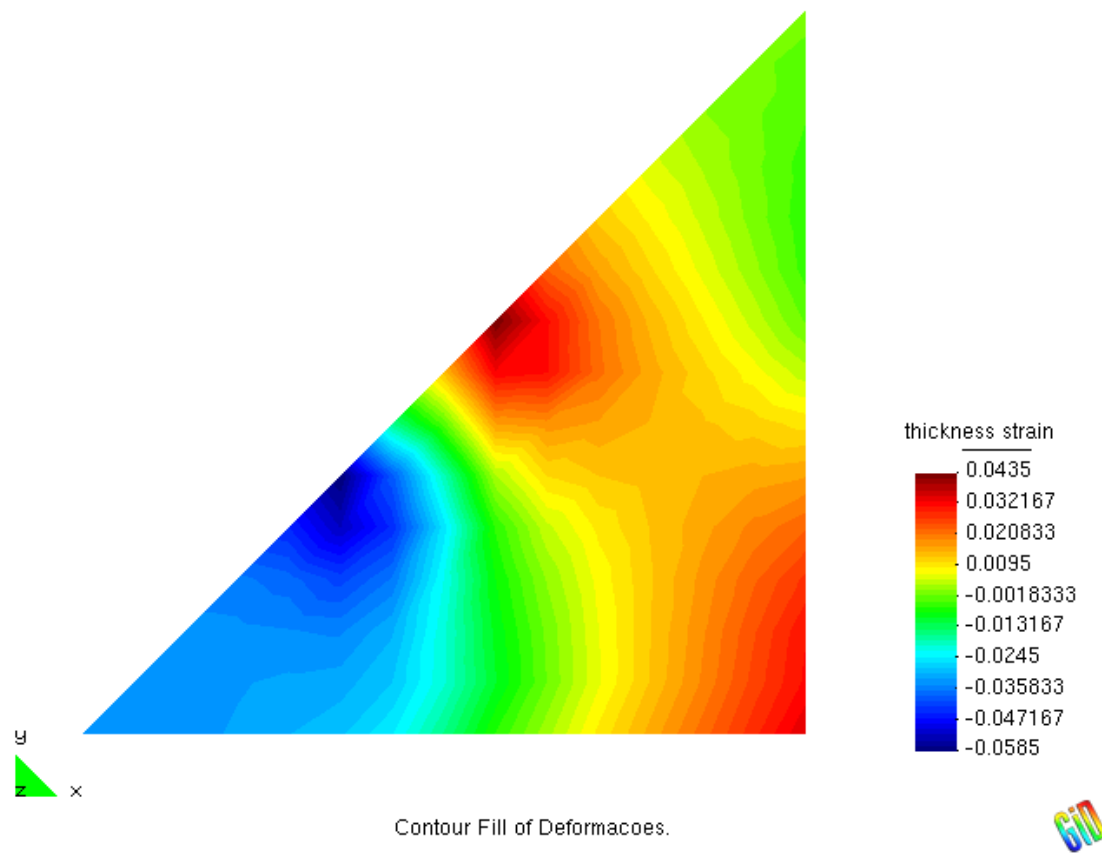

b)

Figura 69: Distribuição da deformação na espessura (profundidade $15 \mathrm{~mm}$ ): a) $M=1,65$ e b) $M=2,0$ 


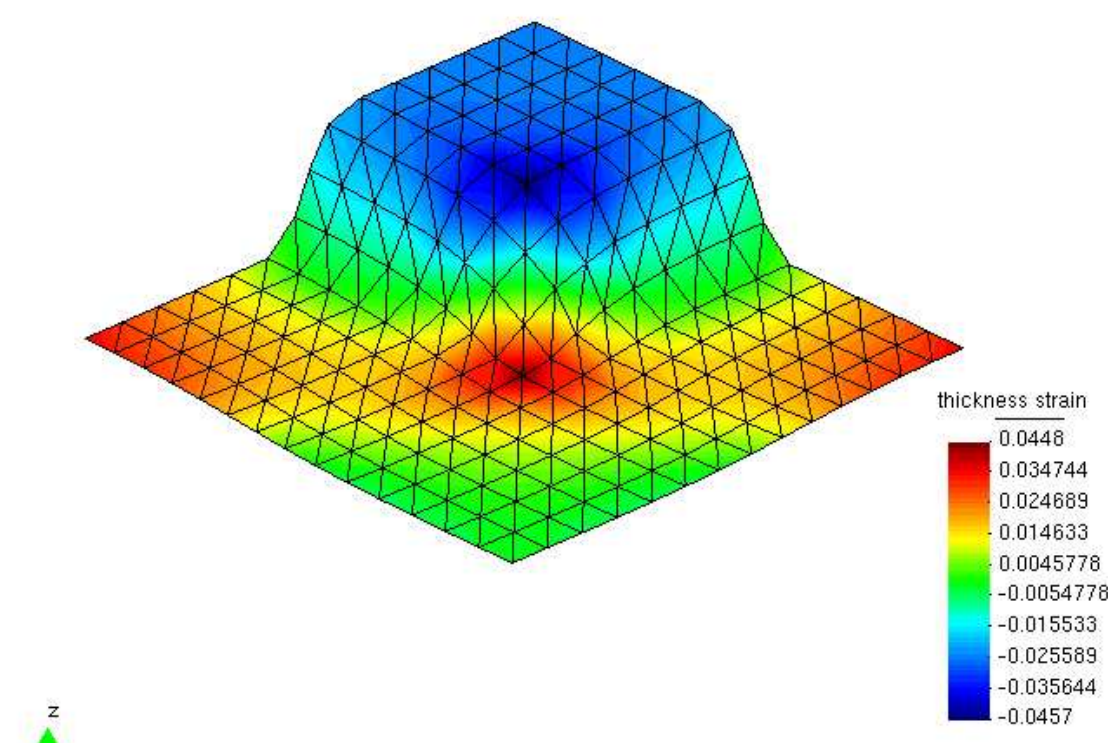

Contour Fill of Deslocamentos, |Deslocamentos|. Deformation (x1): Deslocamentos of Analise, step 1.

a)

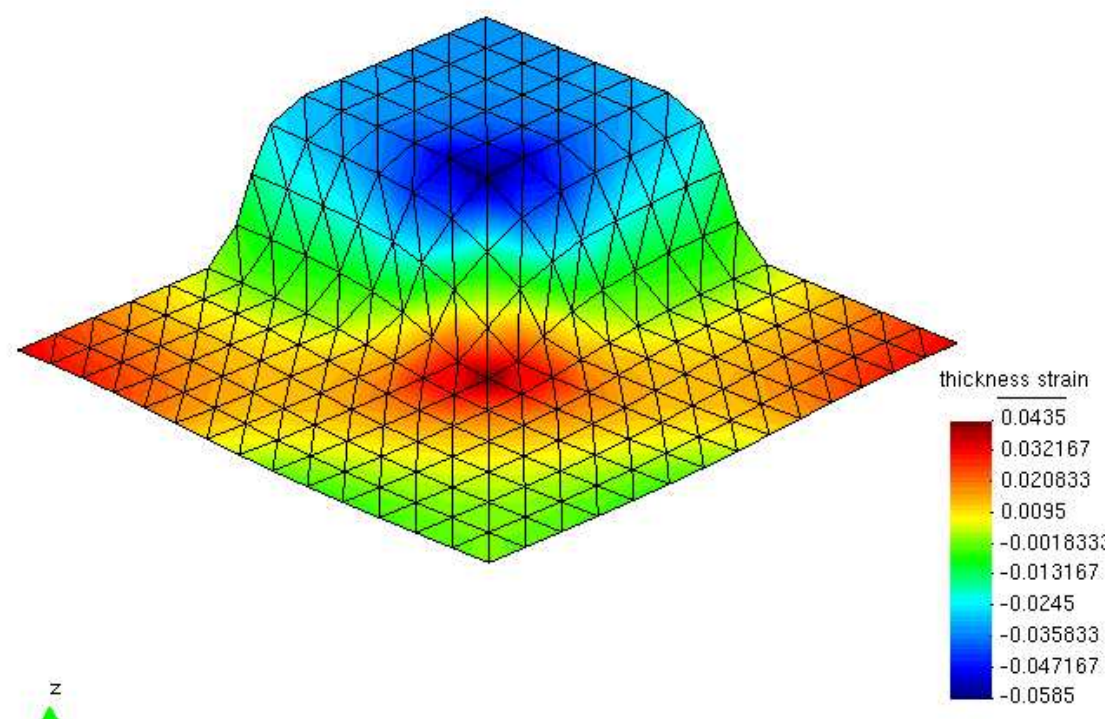

Contour Fill of Deslocamentos, |Deslocamentos|. Deformation (x1): Deslocamentos of Analise, step 1.

b)

Figura 70: Distribuição da deformação na espessura na peça final (profundidade $15 \mathrm{~mm}$ ): a) $M=1,65$ e b) $M=2,0$ 


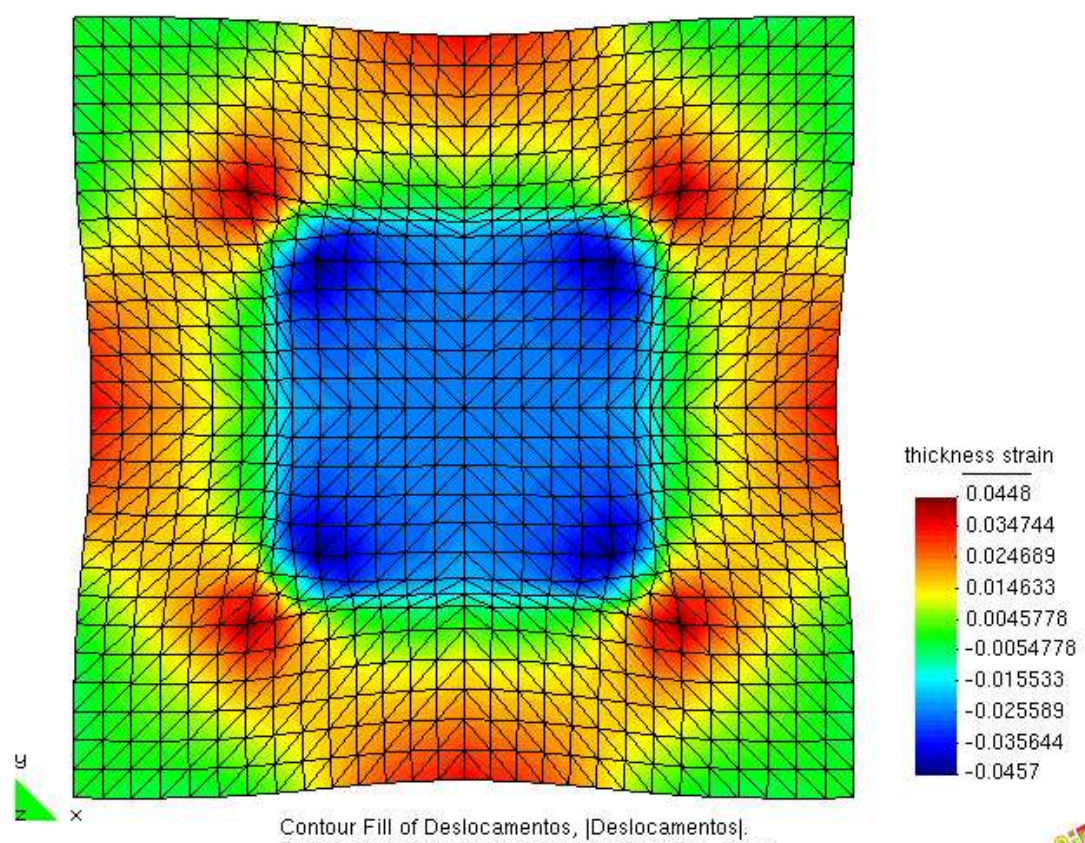

Contour Fill of Deslocamentos, |Deslocamentos|. Deformation (x1): Deslocamentos of Analise, step 1.

a)

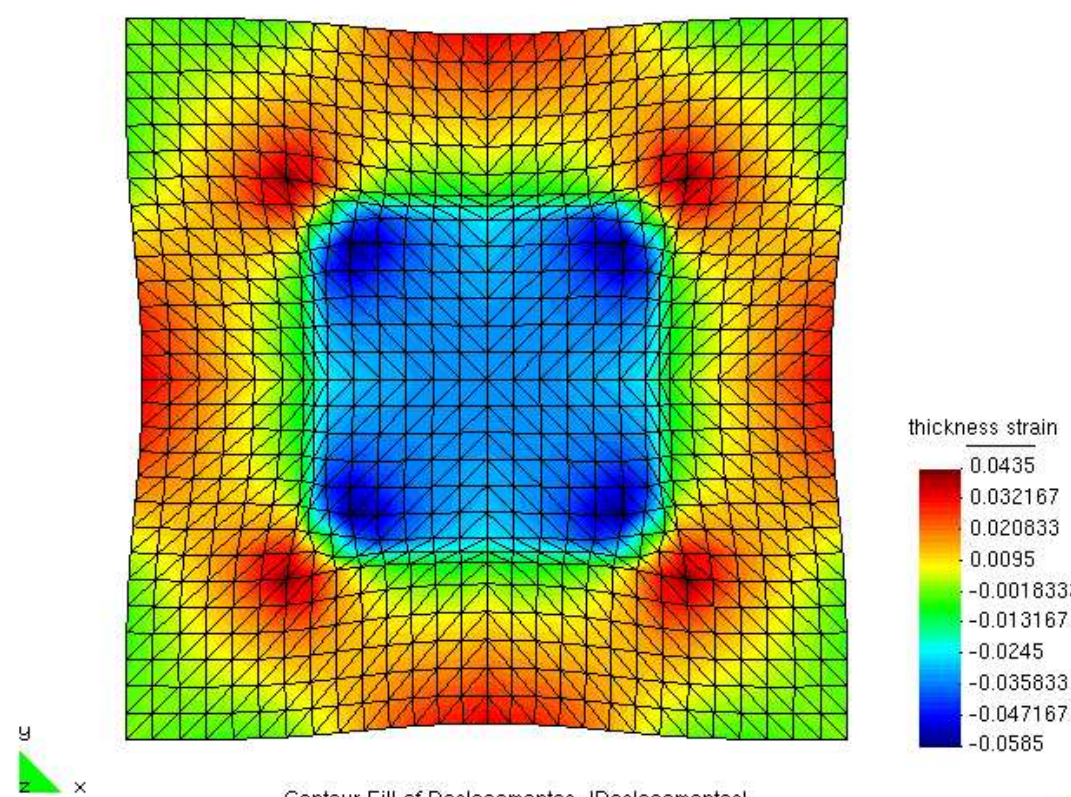

Contour Fill of Deslocamentos, |Deslocamentos|.

Deformation (x1): Deslocamentos of Analise, step 1.

b)

Figura 71: Formação de orelhas (profundidade $15 \mathrm{~mm}$ ): a) $M=1,65$ e b) $M=2,0$ 


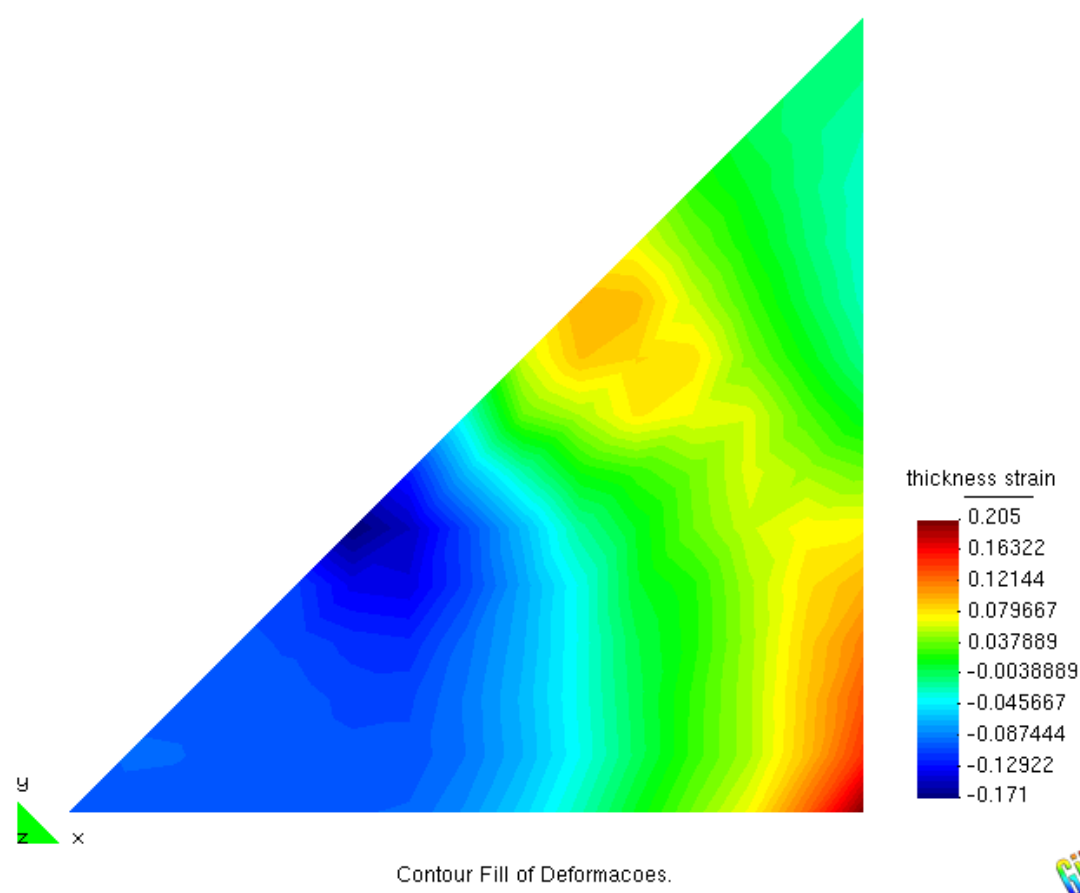

a)

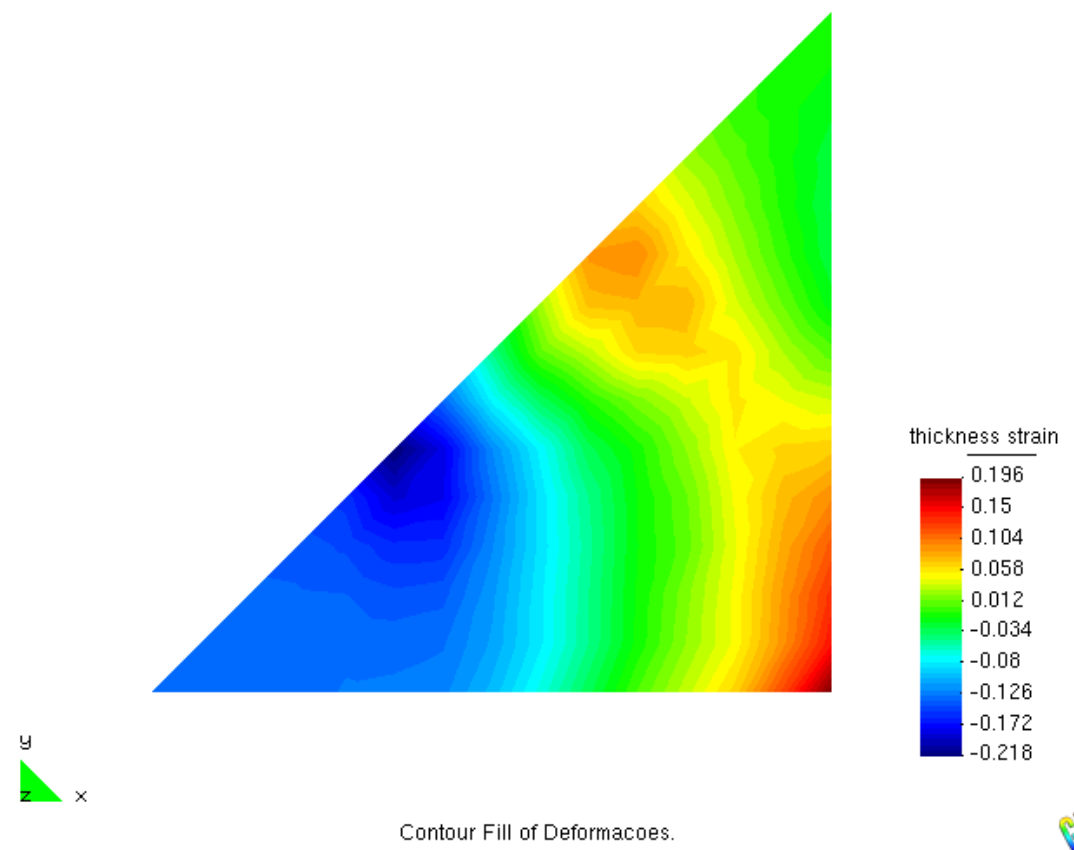

b)

Figura 72: Distribuição da deformação na espessura (profundidade 40mm): a) $M=1,65$ e b) $M=2,0$ 


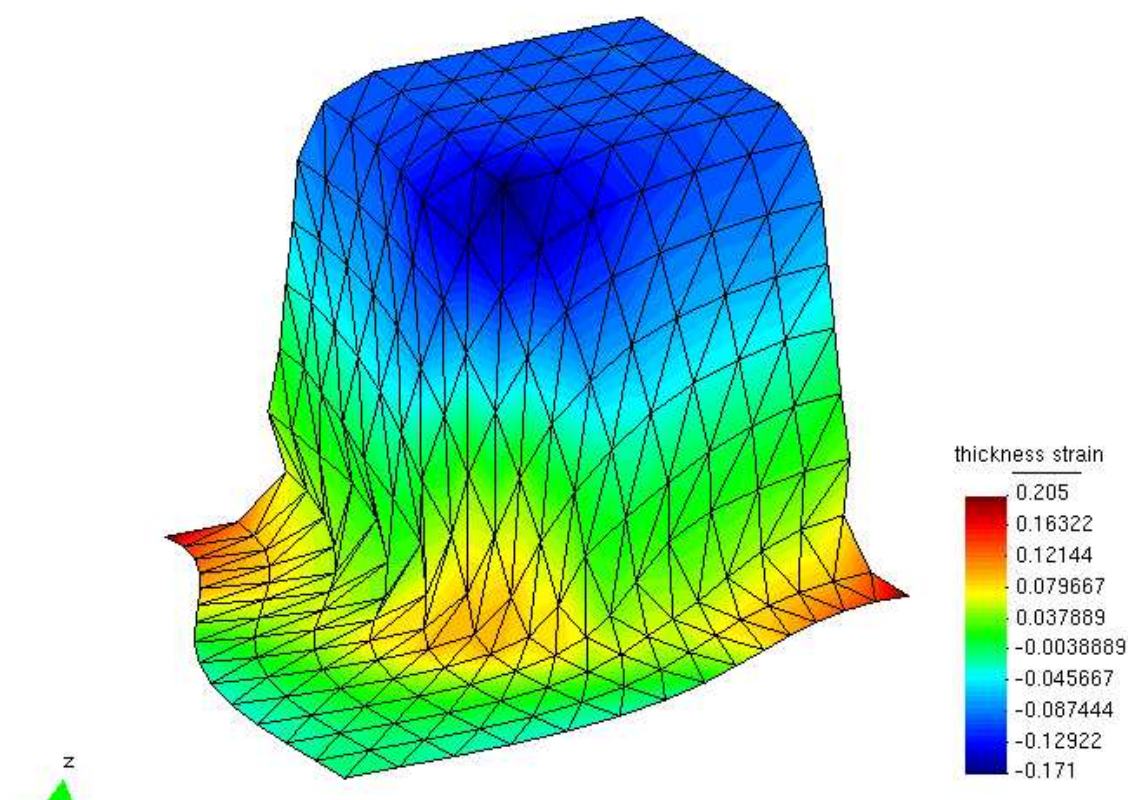

Contour Fill of Deslocamentos, |Deslocamentos|. Deformation ( $x 1$ ): Deslocamentos of Analise, step 1.

a)

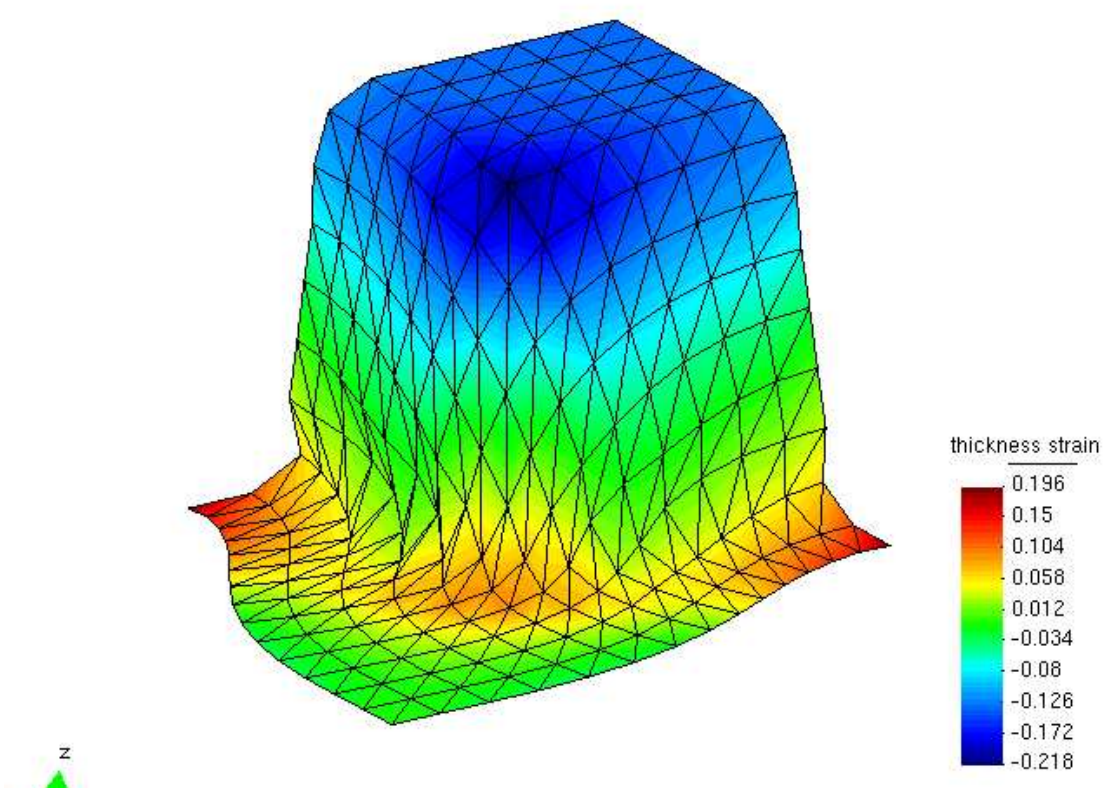

Contour Fill of Deslocamentos, |Deslocamentos|. Deformation ( $x 1$ ): Deslocamentos of Analise, step 1.

b)

Figura 73: Distribuição da deformação na espessura na peça final (profundidade $40 \mathrm{~mm}$ ): a) $M=1,65$ e b) $M=2,0$ 


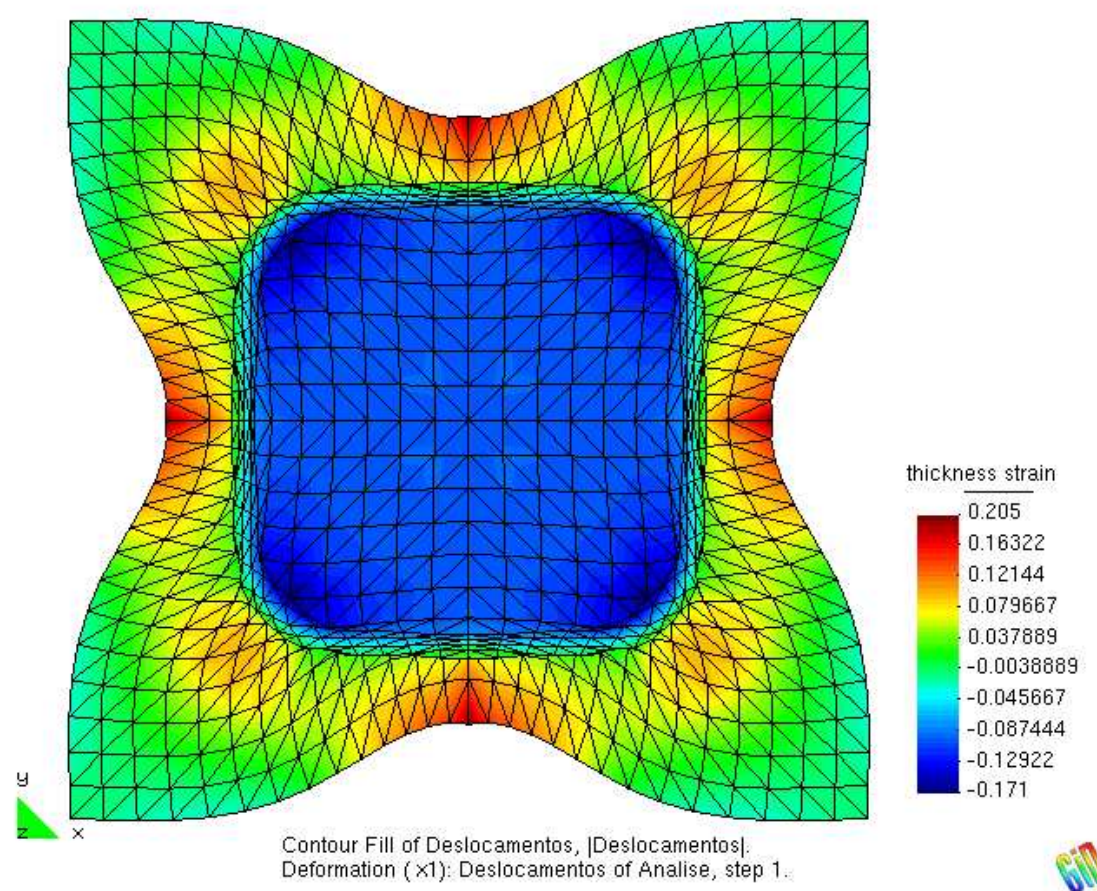

a)

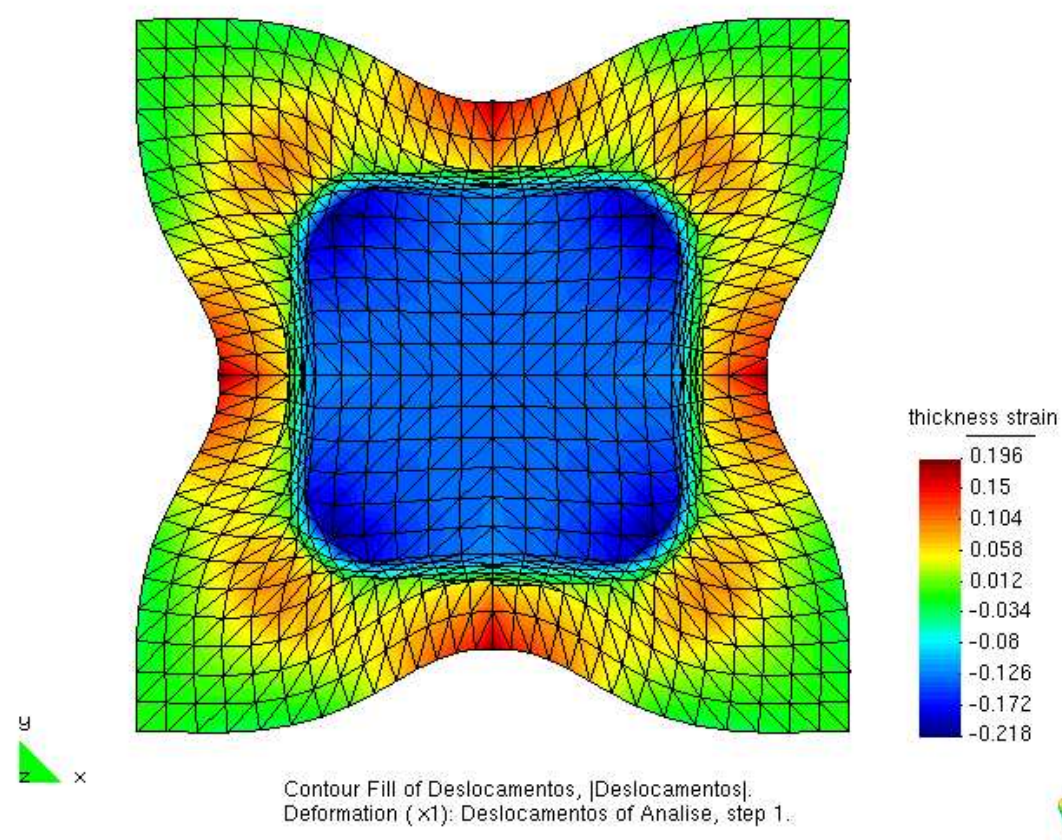

b)

Figura 74: Formação de orelhas (profundidade $40 \mathrm{~mm}$ ): a) $M=1,65$ e b) $M=2,0$ 


\section{Conclusões e Sugestões para Trabalhos Futuros}

\subsection{Conclusões}

O critério de Hill (1979) foi implementado no programa SHEET, obtendo-se bons resultados, coerentes com os apresentados pela literatura disponível, como no estiramento de punção hemisférico de Comstock Jr et al. (2001). Para a estampagem profunda obtiveramse curvas próximas para os dois casos analisados, alumínio e aço doce, e a influência do critério de escoamento na deformação pode ser analisada variando-se o parâmetro $M$ do critério de Hill (1979).

Variando o parâmetro $M$, observaram-se mudanças nos valores das deformações em regiões submetidas a tração biaxial, devido ao alongamento da curva de tensão de escoamento e pelo achatamento da curva de deformação efetiva, provocados pela diminuição do valor de $M$.

O critério de Barlat et al. (1993) não foi implementado, como já dito na página 75 . A implementação demandaria muitas modificações no código fonte do programa SHEET, pois além de implementar o critério de escoamento seria necessário modificar completamente a sub-rotina de cálculo de mudança de coordenadas global-local, afim de considerar a rotação dos eixos de anisotropia, para cada passo de deformação. Assumindo a isotropia planar, o programa SHEET pode simplificar cálculos diversos, não só a translação de coordenadas, mas também partes dos calculos de deformação dos elementos.

A implementação do critério de Hill (1979) mostrou a importância do critério de 
escoamento na análise de conformação de chapas e que, portanto, a escolha de um critério adequado leva a obtenção de resultados mais próximos do real, podendo assim prever falhas e defeitos com maior precisão.

\subsection{Sugestões para Trabalhos Futuros}

Como sugestão para trabalhos futuros, fica a modificação da sub-rotina de translação de coordenadas, habilitando o programa a incluir com mais facilidade critérios de escoamentos com anisotropia planar, como o de Barlat et al. (1993).

Outro ponto a ser explorado seria a implementação de elementos sensíveis a flexão, como o elemento de casca, que possui graus de liberdade de rotação, ou elemento de membrana com flexão, que adicionam o efeito da flexão entre elementos sem acrescentar graus de liberdades a mais. Estes seriam muito úteis na análise da estampagem profunda, já que os elementos de membrana não levam em conta o dobramento que ocorre nos raios do punção e da matriz. 


\section{Referências}

BARLAT, F. et al. Linear transformation-based anisotropic yield functions. International Journal of Plasticity, v. 21, p. 1009-1039, 2005.

BARLAT, F. et al. Plane stress yield function for aluminum alloy sheets. International Journal of Plasticity, v. 19, p. 1297-1319, 2003.

BARLAT, F.; CHUNG, K. Anisotropic potentials for platically deforming metals. Modelling Simul. Mater. Sci. Eng, v. 1, p. 403, 1993.

BARLAT, F.; CHUNG, K.; RICHMOND, O. Plastic dual potential for fcc metals and application to minimum plastic work path calculations. International Journal Plasticity, v. 9, p. 51, 1993.

BARLAT, F.; LEGE, D.; BREM, J. A six-component yield function for anisotropic materials. International Journal Plasticity, v. 7, p. 693, 1991.

BARLAT, F.; LIAN, J. Plastic behavior and stretchability of sheet metals. part 1: Yield function for orthotropic sheets under plane stress conditions. International Journal of Plastics, v. 5, p. 51, 1989.

BARLAT, F. et al. Yield function development for aluminum alloy sheets. Journal of the Mechanics and Physics of Solids, v. 45, n. 11-12, p. 1727-1763, 1997.

BASSANI, J. L. Yield caracterization of metals with transversely isotropic plastic properties. International Journal of Mechanical Sciences, v. 19, p. 651, 1977.

CARDOSO, R. P. R. et al. Development of a one point quadrature shell element for nonlinear applications with contact and anisotropy. Computer Methods in Applied Mechanics and Engineering, v. 191, p. 5177-5206, 2002.

CHEN, C. C. Tese (Doutorado) - University of California, Berkeley, 1978.

CHEN, W. F.; HAN, D. J. Plasticity for Structural Engineers. New York: Springer-Verlag New York Inc., 1988.

CHUNG, K. et al. Blank design for a sheet forming application using the anisotropic strain-rate potential srp98. In: AL, Z. et (Ed.). The Integration of Material, Process and Product Design. Rotterdam: [s.n.], 1999. p. 213-219.

CHUNG, K. et al. Finite element simulation of sheet forming based on a planar anisotropic strain-rate potential. International Journal of Plasticity, v. 12, p. 93, 1996.

CHUNG, K.; SHAH, K. Finite element simulation of sheet metal forming for planar anisotropic metals. International Journal Plasticity, v. 8, p. 453, 1992. 
CHUNG, K.; YOON, J. W.; RICHMOND, O. Ideal sheet forming with frictional constraints. International Journal of Plasticity, v. 16, p. 595-610, 2000.

COMSTOCK JR, E. J.; LI, K.; WAGONER, R. H. Simulation of axisymmetric sheet forming tests. Journal of Materials Processing Technology, v. 117, p. 153-168, 2001.

DAHLQUIST, G. Numerical Methods. [S.l.]: Prentice Hall, 1974.

DANCKERT, J. Experimental investigation of square-cup deep-drawing process. Journal of Materials Processing Technology, v. 50, p. 375-384, 1995.

GAN, W.; WAGONER, R. H. Die desing method for sheet springback. International Journal of Mechanical Sciences, v. 46, n. 7, p. 1097-1113, 2004.

GERMAIN, Y.; CHUNG, K.; WAGONER, R. H. A rigid-viscoplastic finite element program for sheet metal forming analysis. International Journal of Mechanical Sciences, v. 31, n. 1, p. 1-24, 1989.

GOTOH, M. A theory of plastic anisotropy based on a yield function of fourth order-1 and 2. International Journal of Mechanical Sciences, v. 19, p. 505, 1977.

GUO, Y. Q. et al. Finite element procedures for strain estimations of sheet metal forming parts. International Journal for Numerical Methods in Engineering, v. 30, n. 8, p. 1385-1401, 1990.

HILL, R. The Mathematical Theory of Plasticity. England: Oxford Univ. Press., 1950.

HILL, R. Theorical plasticity of textured aggregates. Mathematical Proceedings Cambridge Philosophical Society., v. 85, p. 179, 1979.

HOSFORD, W. F. A generalized isotropic yield function. Journal of Applied Mechanics, v. 39 , p. $607,1972$.

KIM, D. et al. Non-quadratic anisotropic potentials based on linear transformation of plastic strain rate. International Journal of Plasticity, v. 23, p. 1380-1399, 2007.

KIM, J. H.; KOBAYASHI, S. Deformation analysis of axisymmetric sheet metal forming by the rigid-plastic finite element method. In: KOISTINEN, D. P.; WANG, N. M. (Ed.). Mechanics of Sheet Metal Forming. New York: Plenum Press, 1978. p. 341.

KOBAYASHI, S.; OH, I. S.; ALTAN, T. Metal Forming and the Finite-Element Method. Oxford, N.Y., U.S.A: Oxford University Press, 1989.

LIAN, J.; ZHOU, D.; BAUDELET, B. Application of hill's new yield theory to sheet metal forming-part i. hill's 1979 criterion and its application to predicting sheet forming limit. Internacional Journal of Mechanical Science, v. 31, n. 4, p. 237-247, 1989.

MACHINOUCHI, A. (Ed.). Proceedings of the 2nd International Conference NUMISHEET 93. Isehara, Japan: [s.n.], 1993.

MANABE, K. et al. Validation of fe simulation based on surface roughness model in micro-deep drawing. Journal of Materials Processing Technology, v. 204, n. 1-3, p. 89-93, 2008. 
MARCINIAK, Z.; DUNCAN, J. L. The Mechanics of Sheet Metal Forming. Great Britain: Edward Arnold, a division of Hodder and Stoughton Limited, 1992.

MARCINIAK, Z.; DUNCAN, J. L.; HU, S. J. The Mechanics of Sheet Metal Forming. Great Britain: Edward Arnold, a division of Hodder and Stoughton Limited, 2002.

MEHTA, H. S.; KOBAYASHI, S. Finite Analysis and Experimental Investigation of Sheet Metal Stretching. University of California, 1971.

OLIENICK FILHO, E. G. Análise da estampagem de chapas metálicas pelo método dos elementos finitos. Dissertação (Mestrado) - PPGEM/CEFET-PR, Curitiba - PR, 2003.

ONATE, E.; ZIENKIEWICS, O. C. A viscous shell formulation for the analysis of thin sheet metal forming. International Journal of Mechanical Sciences, v. 25, n. 5, p. 305-335, 1983.

PARMAR, A.; MELLOR, P. B. Predictionsof limit strains of sheet metal using a more general yield criterion. Internationa Journal of Mechanical Science, v. 20, p. 385, 1978.

RODRIGUES, J.; MARTINS, P. Tecnologia Mecânica. [S.l.]: Escolar Editora, 2005.

TEKKAYA, A. E. State-of-the-art of simulation of sheet metal forming. Journal of Materials Processing Technology, v. 103, p. 14-22, 2000.

TOH, C. H. Process Modeling of Sheet Metal Forming of General Shapes by the Finite Element Method Based on Large Strain Formulation. Tese (Doutorado) — Graduate Division of the University of California, Berkeley, USA, 1983.

WANG, N. M. A rigid-plastic rate-sensitive finite element method for modeling sheet metal forming processes. In: PITTMAN, J. F. T. et al. (Ed.). Numerical analysis of forming processes. [S.l.]: Wiley, 1984. cap. 5, p. 117.

WANG, N. M.; BUDIANSKKY, B. Analysis of sheet metal by a finite element method. Journal of Applied Mechanics, v. 45, p. 73-83, 1978.

WIFI, A. S. An incremental complete solution of the stretch-forming and deep drawing of a circular blank using a hemispherical punch. International Journal of Mechanical Science, v. 18, p. 23, 1976.

WOO, D. M. On the complete solution of the deep-drawing problem. International Journal of Mechanical Sciences, v. 10, p. 83-94, 1968.

WOODTHORPE, J.; PEARCE, R. The anomalous behaviour of aluminium sheet under balance biaxial tension. Internacional Journal of Mechanical Science, v. 12, p. 341, 1970.

YOO, D. J. et al. Rigid-plastic finite element analysis of sheet metal forming processes using continuous contact treatment and membrane elements incorporating bending effects. International Journal of Mechanical Sciences, v. 36, n. 6, p. 513-546, 1994.

YOON, J. et al. Prediction of six or eight ears in a drawn cup based on a new anisotropic yield function. International Journal of Plasticity, v. 22, p. 174-193, 2006. 
YOON, J. W. et al. Finite element method for sheet forming based on an anisotropic strain-rate potential and the convected coordinate system. Internacional Journal of Mechanical Science, v. 37, n. 7, p. 733, 1995.

YOON, J. W.; YANG, D. Y.; CHUNG, K. Elasto-plastic finite element method based on incremental deformation theory and continuum based shell elements for planar anisotropic sheet materials. Computer Methods in Applied Mechanics and Engineering, v. 174, p. 23-56, 1999. 


\section{ANEXOA - Sub-rotinas Modificadas}

A seguir estão listados as sub-rotinas modificadas para a inclusão do critério de escoamento de Hill (1979). O programa completo se encontra em Toh (1983).

\section{A.1 Sub-rotina ASEMBL}

SUBROUTINE ASEMBL (ISTEP, X, Y , Z, U, V , W , FTX , FTY , FTZ, IE, EFSTRN,

\$ EX , EY, EXY , TAUO , FNX , FNY, FNZ, DEF , XL , YL , ZL , UL , VL , WL , EXZ, EYZ, EZ,

2 XO, YO, EFSTRO, EFSTRT, EFO, EFF, DEFO, TSTRAN , TSTRAO)

INITIALIZE MATRICES' COMPUTE ELEMENTAL QUANTITIES AND ASSEMBLE

THE OVERALL ELEMENTAL STIFFNESS MATRICES

DIMENSION U(1), V(1),W(1), FTX (1), FTY(1), FTZ(1), FF(9), Q (9),

$\$ \quad B(10,10), \operatorname{BT}(10,10), B B(10,10), Q K(10,10), \operatorname{IE}(4,1), \operatorname{LP}(9)$

$2 \quad, X(1), Y(1), Z(1), \operatorname{EFSTRN}(1), \operatorname{EX}(1), \operatorname{EY}(1), \operatorname{EXY}(1), \operatorname{TAUO}(1)$,

$3 \operatorname{BUU}(4,9), \operatorname{BU}(4,1), \operatorname{BTU}(9,4,9), \operatorname{BTBUU}(9,9), \operatorname{BTUBU}(9,9)$

$4 \quad, \operatorname{FNX}(1), \operatorname{FNY}(1), \operatorname{FNZ}(1), \operatorname{DEF}(1), \mathrm{XL}(1), \mathrm{YL}(1), \mathrm{ZL}(1), \mathrm{XO}(1), \mathrm{YO}(1)$

$5 \quad, \mathrm{UL}(1), \operatorname{VL}(1), \operatorname{WL}(1), \operatorname{ETA}(3,3), \operatorname{BTETA}(9,9), \operatorname{BTETAU}(9,9)$

$6 \quad, \operatorname{ETABU}(9,9), \operatorname{BEBOST}(9,9), \operatorname{EXZ}(1), \operatorname{EYZ}(1), \operatorname{EZ}(1)$

$7 \quad, \operatorname{ETAB}(4,1), \operatorname{ETAC}(9,9), \operatorname{ETACT}(9,9), \operatorname{QK} 1(9,9), \mathrm{QK} 2(9,9)$

$8 \quad, \operatorname{QK} 3(9,1), \operatorname{EFO}(1), \operatorname{EFF}(1), \operatorname{EFSTRO}(1), \operatorname{EFSTRT}(1), \operatorname{DEFO}(1)$,

$9 \operatorname{TStran}(1), \operatorname{TStRaO}(1), \operatorname{TTT}(9), \operatorname{TTdU}(9,9), \operatorname{ETAD} 1(3,3), \operatorname{BTUETAd} 1(9,9)$

A $\quad, \operatorname{BTETAD} 1(9,9), \operatorname{VQK}(9,9), \operatorname{BTUBU} 1(9,9), \operatorname{VQK} 1(9,9)$

B

C

COMMON/ONE/ MAXNP, MAXEL, MAXMAT, ICLASS 
COMMON/TWO/ NNP, NEL, NEQ, SIGO(1)

COMMON/FIVE/ THC, FMDI , XN , RA, WIDTH, XENGTH , FM

COMMON/THREE/R (900), AK (900, 900), HH (900)

COMMON/FORN/ SAVES $(900,900)$

COMMON/FOUR/ TO, DT, TT, DTO

COMMON/TRAN/ D1, D2 , D3 , D4 , D5, D6, D7, D8, D9

COMMON/CMAT/ CONT1, CONT2, RVALUE

COMMON/HILL79/ MH

REAL MH

DO $50 \quad I=1, N E Q$

$\mathrm{HH}(\mathrm{I})=0.0$

$R(I)=0.0$

DO $50 \mathrm{~J}=1$, NEQ

50

$$
\operatorname{AK}(I, J)=0.0
$$

! COMPUTE ELEMENT STIFFNESS MATRICES AND LOADS AND SYSTEM MATRICES

DO $60 \mathrm{M}=1$, NEL

$L L=\operatorname{IE}(1, M)$

$\mathrm{MM}=\operatorname{IE}(2, \mathrm{M})$

$\mathrm{NN}=\operatorname{IE}(3, \mathrm{M})$

CALL TRANFM (LL , MM , NN , X, Y , Z, XL, YL , ZL , UL, VL, WL , U , V , W)

D0 $600 \quad I=1,9$

DO $600 \mathrm{~J}=1,9$

$\operatorname{ETAC}(I, J)=0.0$

600

CONTINUE

$\mathrm{B} 1=\mathrm{YL}(\mathrm{MM})-\mathrm{YL}$ (NN)

$B 2=Y L(N N)-Y L(L L)$

$B 3=Y L(L L)-Y L(M M)$

$\mathrm{C} 1=\mathrm{XL}(\mathrm{NN})-\mathrm{XL}(\mathrm{MM})$

$\mathrm{C} 2=\mathrm{XL}(\mathrm{LL})-\mathrm{XL}(\mathrm{NN})$

$\mathrm{C} 3=\mathrm{XL}(\mathrm{MM})-\mathrm{XL}(\mathrm{LL})$

! GLOBAL NODAL POINT FORCE COMPONENTS

$\mathrm{FF}(1)=\mathrm{FTX}(\mathrm{LL})+\mathrm{FNX}(\mathrm{LL})$

FF (2) $=$ FTY (LL) $+F N Y(L L)$

$\mathrm{FF}(3)=\mathrm{FTZ}(\mathrm{LL})+\mathrm{FNZ}(\mathrm{LL})$

FF (4) $=$ FTX (MM) +FNX (MM)

$F F(5)=F T Y(M M)+F N Y(M M)$ 
$F F(6)=F T Z(M M)+F N Z(M M)$

$\mathrm{FF}(7)=\mathrm{FTX}(\mathrm{NN})+\mathrm{FNX}(\mathrm{NN})$

$\mathrm{FF}(8)=\mathrm{FTY}(\mathrm{NN})+\mathrm{FNY}(\mathrm{NN})$

$\mathrm{FF}(9)=\mathrm{FTZ}(\mathrm{NN})+\mathrm{FNZ}(\mathrm{NN})$

CALL STRAIN (M, LL , MM, NN , XL , YL , UL , VL, WL , DELTA , TO , DT , ESO , BH , EFSTRN

\$ , TT , EFO , EX , EY , EXY , TAUO , EFF , DEF , EXZ, EYZ, EZ, EFSTRO, EFSTRT, TSTRAN

2 ,DEFO, DTO, TSTRAO)

ELEMENT MATRIX B

IF(DELTA.LE.0.0) GOTO 916

$\mathrm{DELTO}=\mathrm{XO}(\mathrm{MM}) * \mathrm{YO}(\mathrm{NN})-\mathrm{YO}(\mathrm{MM}) * \mathrm{XO}(\mathrm{NN})-\mathrm{XO}(\mathrm{LL}) * \mathrm{YO}(\mathrm{NN})+\mathrm{XO}(\mathrm{LL}) * \mathrm{YO}(\mathrm{MM})+$

$\$$ $\mathrm{XO}(\mathrm{NN}) * \mathrm{YO}(\mathrm{LL})-\mathrm{XO}(\mathrm{MM}) * \mathrm{YO}(\mathrm{LL})$

IF (DELTO.LE.0.0) GOTO 916

IF (ISTEP.GT.1) DELTO=DELTA

$\mathrm{RFT}=2.0 / 3.0$

IF (ICLASS.EQ . 4 . AND . ABS (EFSTRN (M)) .1E.1.00E-20)

$\$ \quad \operatorname{EFSTRN}(\mathrm{M})=1.00 \mathrm{E}-15$

$\operatorname{IF}(\operatorname{ICLASS} \cdot E Q \cdot 5 \cdot \operatorname{AND} \cdot \operatorname{EFSTRN}(M) \cdot E Q \cdot 0 \cdot 0) \operatorname{EFSTRN}(M)=1 \cdot 00 \mathrm{E}-15$

IF (ICLASS . EQ . 6 AND . EFSTRN (M) . EQ . 0 .0) $\operatorname{EFSTRN~(M)~}=1 \cdot 00 \mathrm{E}-15$

!

FIX1 $=2 . * *(1 . / \mathrm{MH}-1) *.(1 .+\mathrm{RVALUE}) * *(1 . / \mathrm{MH})$

EFFTT $=$ EFSTRN $(M)$

$\operatorname{EFFTT}=\operatorname{EFSTRN}(\mathrm{M}) * *(\mathrm{MH} /(\mathrm{MH}-1).) / \mathrm{FIX} 1$

VOLU $=$ THC $*$ DELTO $/ 2.0 * \operatorname{EXP}($ EFSTRO $(\mathrm{M}))$

FXITORTT $=F I X 1 *(M H-1$.$) / MH * E F F T T * *(-1 . / M H) *(E S O+B H * E F S T R N(M))$

IF (EFFTT.LT.1.00E-15) FXITORGRAD=0.0

IF (EFFTT .LT.1.00E-15) FXITORPROD=0.0

! NEAR-RIGID ELEMENT TREATMENT FOR SQUARE CUP DRAWING

RIGID $=0.005$

IF (ICLASS . GE . 5 . AND . EFSTRN (M) .LE. RIGID) FXITORTT=FIX1* (MH-1.)

$\$ \quad / M H * E F F T T * *(-1 . / M H) *(E S O / R I G I D+B H) * E F S T R N(M)$

! 
$\mathrm{AA}=1.0 /(2.0 * \mathrm{DELTA} * \mathrm{DELTA})$

$\mathrm{XBU}=\mathrm{B} 1 * \mathrm{UL}(\mathrm{LL})+\mathrm{B} 2 * \mathrm{UL}(\mathrm{MM})+\mathrm{B} 3 * \mathrm{UL}(\mathrm{NN})$

$\mathrm{XCU}=\mathrm{C} 1 * \mathrm{UL}(\mathrm{LL})+\mathrm{C} 2 * \mathrm{UL}(\mathrm{MM})+\mathrm{C} 3 * \mathrm{UL}(\mathrm{NN})$

$\mathrm{XBV}=\mathrm{B} 1 * \mathrm{VL}(\mathrm{LL})+\mathrm{B} 2 * \mathrm{VL}(\mathrm{MM})+\mathrm{B} 3 * \mathrm{VL}(\mathrm{NN})$

$\mathrm{XCV}=\mathrm{C} 1 * \mathrm{VL}(\mathrm{LL})+\mathrm{C} 2 * \mathrm{VL}(\mathrm{MM})+\mathrm{C} 3 * \mathrm{VL}(\mathrm{NN})$

$\mathrm{XBW}=\mathrm{B} 1 * \mathrm{WL}(\mathrm{LL})+\mathrm{B} 2 * \mathrm{WL}(\mathrm{MM})+\mathrm{B} 3 * \mathrm{WL}(\mathrm{NN})$

$\mathrm{XCW}=\mathrm{C} 1 * \mathrm{WL}(\mathrm{LL})+\mathrm{C} 2 * \mathrm{WL}(\mathrm{MM})+\mathrm{C} 3 * \mathrm{WL}(\mathrm{NN})$

$B(1,1)=A * B 1+A A * B 1 * X B U$

$\mathrm{B}(1,2)=\mathrm{AA} * \mathrm{~B} 1 * \mathrm{XBV}$

$\mathrm{B}(1,3)=\mathrm{AA} * \mathrm{~B} 1 * \mathrm{XBW}$

$\mathrm{B}(1,4)=\mathrm{A} * \mathrm{~B} 2+\mathrm{AA} * \mathrm{~B} 2 * \mathrm{XBU}$

$\mathrm{B}(1,5)=\mathrm{AA} * \mathrm{~B} 2 * \mathrm{XBV}$

$\mathrm{B}(1,6)=\mathrm{AA} * \mathrm{~B} 2 * \mathrm{XBW}$

$B(1,7)=A * B 3+A A * B 3 * X B U$

$\mathrm{B}(1,8)=\mathrm{AA} * \mathrm{~B} 3 * \mathrm{XBV}$

$B(1,9)=A A * B 3 * X B W$

$\mathrm{B}(2,1)=\mathrm{AA} * \mathrm{C} 1 * \mathrm{XCU}$

$\mathrm{B}(2,2)=\mathrm{A} * \mathrm{C} 1+\mathrm{AA} * \mathrm{C} 1 * \mathrm{XCV}$

$\mathrm{B}(2,3)=\mathrm{AA} * \mathrm{C} 1 * \mathrm{XCW}$

$\mathrm{B}(2,4)=\mathrm{AA} * \mathrm{C} 2 * \mathrm{XCU}$

$B(2,5)=A * C 2+A A * C 2 * X C V$

$\mathrm{B}(2,6)=\mathrm{AA} * \mathrm{C} 2 * \mathrm{XCW}$

$\mathrm{B}(2,7)=\mathrm{AA} * \mathrm{C} 3 * \mathrm{XCU}$

$\mathrm{B}(2,8)=\mathrm{A} * \mathrm{C} 3+\mathrm{AA} * \mathrm{C} 3 * \mathrm{XCV}$

$\mathrm{B}(2,9)=\mathrm{AA} * \mathrm{C} 3 * \mathrm{XCW}$

$\mathrm{B}(3,1)=\mathrm{A} * \mathrm{C} 1 / 2.0+\mathrm{AA} * \mathrm{C} 1 * \mathrm{XBU}$

$\mathrm{B}(3,2)=\mathrm{A} * \mathrm{~B} 1 / 2.0+\mathrm{AA} * \mathrm{C} 1 * \mathrm{XBV}$

$\mathrm{B}(3,3)=\mathrm{AA} * \mathrm{C} 1 * \mathrm{XBW}$

$B(3,4)=A * C 2 / 2.0+A A * C 2 * X B U$

$\mathrm{B}(3,5)=\mathrm{A} * \mathrm{~B} 2 / 2 \cdot 0+\mathrm{AA} * \mathrm{C} 2 * \mathrm{XBV}$

$\mathrm{B}(3,6)=\mathrm{AA} * \mathrm{C} 2 * \mathrm{XBW}$

$\mathrm{B}(3,7)=\mathrm{A} * \mathrm{C} 3 / 2,0+\mathrm{AA} * \mathrm{C} 3 * \mathrm{XBU}$

$\mathrm{B}(3,8)=\mathrm{A} * \mathrm{~B} 3 / 2 \cdot 0+\mathrm{AA} * \mathrm{C} 3 * \mathrm{XBV}$

$\mathrm{B}(3,9)=\mathrm{AA} * \mathrm{C} 3 * \mathrm{XBW}$

$\mathrm{AB} 2=\mathrm{AA} * \mathrm{~B} 2$ 


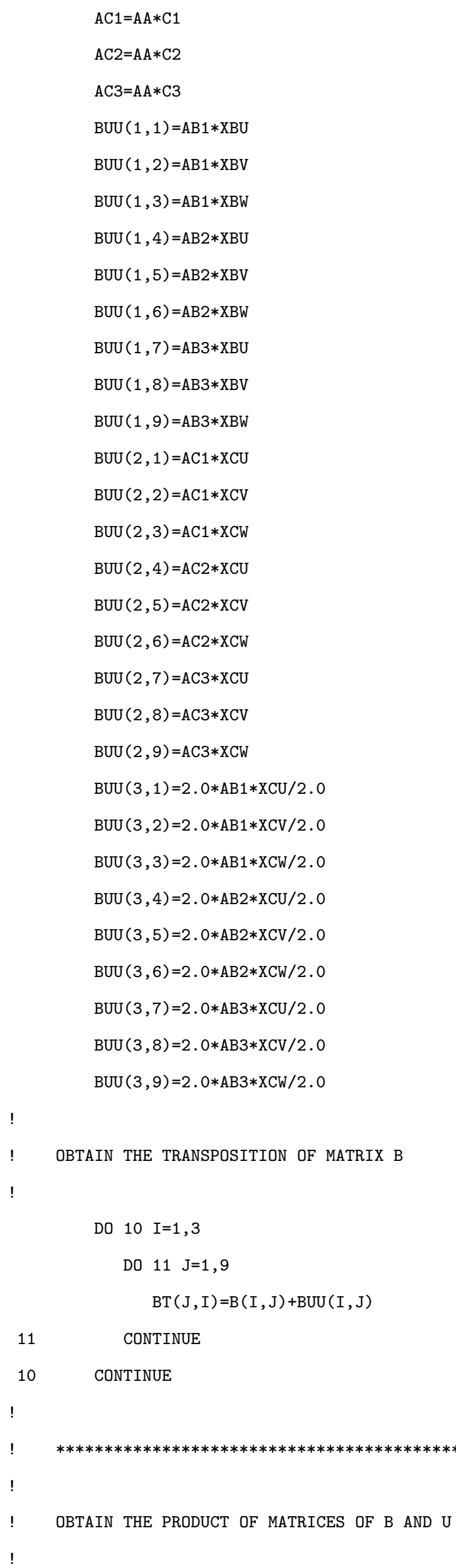


DO $900 \mathrm{~K}=1,3$

$\mathrm{BU}(\mathrm{K}, 1)=\mathrm{B}(\mathrm{K}, 1) * \mathrm{UL}(\mathrm{LL})+\mathrm{B}(\mathrm{K}, 2) * \mathrm{VL}(\mathrm{LL})+\mathrm{B}(\mathrm{K}, 3) * \mathrm{WL}(\mathrm{LL})+\mathrm{B}(\mathrm{K}, 4) * \mathrm{UL}(\mathrm{MM})$

$\$ \quad+\mathrm{B}(\mathrm{K}, 5) * \mathrm{VL}(\mathrm{MM})+\mathrm{B}(\mathrm{K}, 6) * \mathrm{WL}(\mathrm{MM})+\mathrm{B}(\mathrm{K}, 7) * \mathrm{UL}(\mathrm{NN})+\mathrm{B}(\mathrm{K}, 8) * \mathrm{VL}(\mathrm{NN})+$

$2 \quad \mathrm{~B}(\mathrm{~K}, 9) * \mathrm{WL}(\mathrm{NN})$

900 CONTINUE

!

! EVALUATE THE D MATRIX

DO $510 \quad I=1,3$

DO $510 \mathrm{~J}=1,3$

$\operatorname{ETA}(I, J)=0.0$

$\operatorname{ETAD} 1(\mathrm{I}, \mathrm{J})=0.0$

510 CONTINUE

$\operatorname{ETAD} 1(1,1)=1.0$

$\operatorname{ETAD} 1(2,2)=1.0$

$\operatorname{ETAD} 1(1,2)=1.0$

$\operatorname{ETAD} 1(2,1)=1.0$

$\operatorname{ETA}(1,1)=1.0$

$\operatorname{ETA}(1,2)=-1.0$

$\operatorname{ETA}(2,1)=-1.0$

$\operatorname{ETA}(2,2)=1.0$

$\operatorname{ETA}(3,3)=4.0$

DO $520 \mathrm{~L}=1,3$

$\operatorname{ETAB}(L, 1)=0.0$

$\operatorname{ETAB} 1(\mathrm{~L}, 1)=0.0$

DO $540 \mathrm{~N}=1,3$

$\operatorname{ETAB}(\mathrm{L}, 1)=\operatorname{ETAB}(\mathrm{L}, 1)+\operatorname{ETA}(\mathrm{L}, \mathrm{N}) * \mathrm{BU}(\mathrm{N}, 1)$

$\operatorname{ETAB} 1(\mathrm{~L}, 1)=\operatorname{ETAB} 1(\mathrm{~L}, 1)+\operatorname{ETAD} 1(\mathrm{~L}, \mathrm{~N}) * \mathrm{BU}(\mathrm{N}, 1)$

540

CONTINUE

520 CONTINUE

D0 $530 I=1,3$

D0 $550 \mathrm{~J}=1,9$

$\operatorname{BTETA}(I, J)=0.0$

$\operatorname{ETABU}(I, J)=0.0$

$\operatorname{BTETAD} 1(\mathrm{I}, \mathrm{J})=0.0$

$\operatorname{ETAD} 1 B U(I, J)=0.0$

DO $560 \mathrm{~K}=1,3$

$\operatorname{BTETA}(I, J)=\operatorname{BTETA}(I, J)+\operatorname{ETA}(I, K) * B(K, J)$

$\operatorname{ETABU}(I, J)=\operatorname{ETABU}(I, J)+\operatorname{ETA}(I, K) * \operatorname{BUU}(K, J)$

$\operatorname{BTETAD} 1(\mathrm{I}, \mathrm{J})=\operatorname{BTETAD} 1(\mathrm{I}, \mathrm{J})+\operatorname{ETAD} 1(\mathrm{I}, \mathrm{K}) * \mathrm{~B}(\mathrm{~K}, \mathrm{~J})$

$\operatorname{ETAD} 1 B U(I, J)=\operatorname{ETAD} 1 B U(I, J)+\operatorname{ETAD} 1(I, K) * \operatorname{BUU}(\mathrm{K}, \mathrm{J})$ 


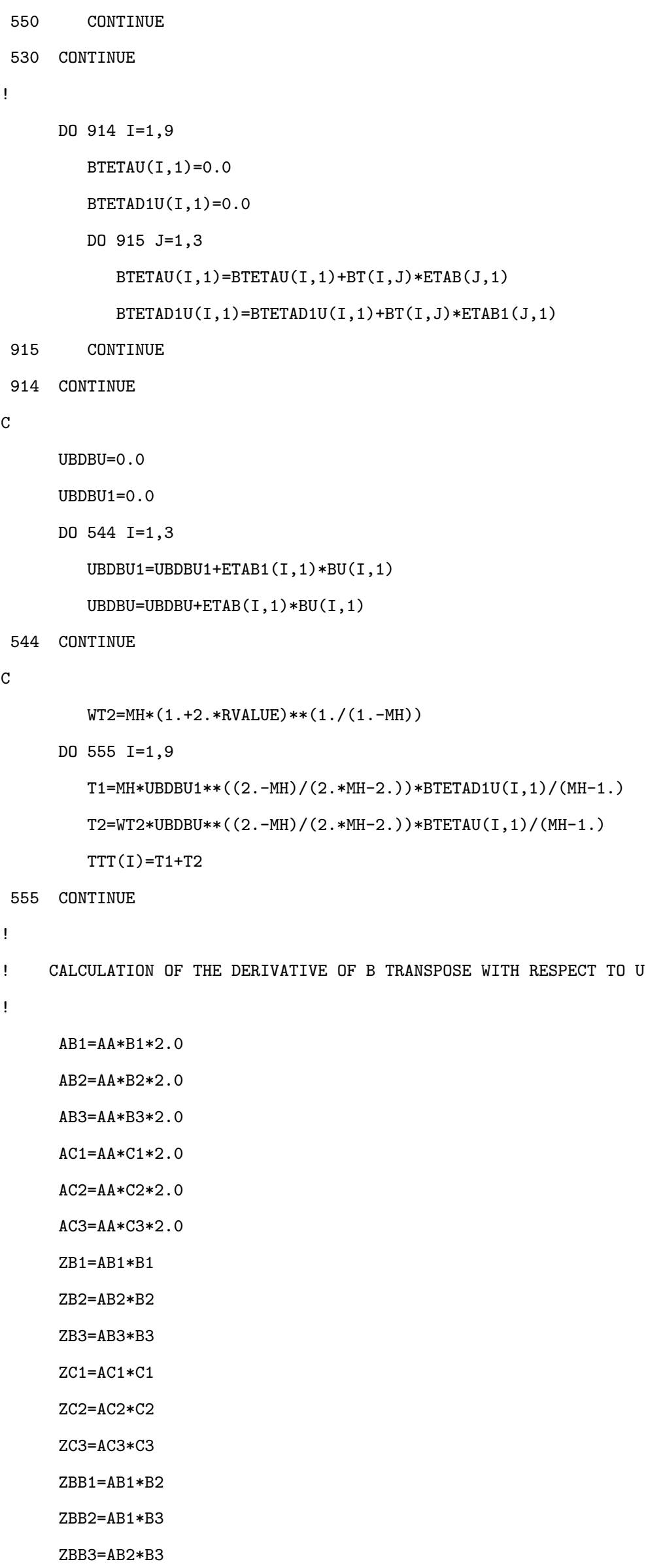


$\mathrm{ZCC} 1=\mathrm{AC} 1 * \mathrm{C} 2$

$\mathrm{ZCC} 2=\mathrm{AC} 1 * \mathrm{C} 3$

$\mathrm{ZCC} 3=\mathrm{AC} 2 * \mathrm{C} 3$

$\mathrm{ZCB} 1=\mathrm{AB} 1 * \mathrm{C} 1$

$\mathrm{ZCB} 2=\mathrm{AB} 2 * \mathrm{C} 1$

$\mathrm{ZCB} 3=\mathrm{AB} 3 * \mathrm{C} 1$

$\mathrm{ZCB} 4=\mathrm{AB} 1 * \mathrm{C} 2$

$\mathrm{ZCB} 5=\mathrm{AB} 2 * \mathrm{C} 2$

$\mathrm{ZCB} 6=\mathrm{AB} 3 * \mathrm{C} 2$

$\mathrm{ZCB} 7=\mathrm{AB} 1 * \mathrm{C} 3$

$\mathrm{ZCB} 8=\mathrm{AB} 2 * \mathrm{C} 3$

$\mathrm{ZCB} 9=\mathrm{AB} 3 * \mathrm{C} 3$

!

DO $901 \mathrm{I}=1,7,3$

$\operatorname{ETAC}(I, I)=D 1$

$\operatorname{ETAC}(I, I+1)=D 2$

$\operatorname{ETAC}(I, I+2)=D 3$

DO $902 \mathrm{~J}=1,3$

DO $903 \mathrm{~K}=2,9$

IF(K.EQ.4.OR.K.EQ.7) GOTO 903

$\operatorname{BTU}(\mathrm{I}, \mathrm{J}, \mathrm{K})=0.0$

903

CONTINUE

CONTINUE

901

DO $904 \mathrm{II}=2,8,3$

$\operatorname{ETAC}(I I, I I-1)=D 4$

$\operatorname{ETAC}(I I, I I)=D 5$

$\operatorname{ETAC}(\mathrm{II}, \mathrm{II}+1)=\mathrm{D} 6$

DO $905 \mathrm{JJ}=1,3$

DO $906 \mathrm{KK}=1,9$

IF (KK.EQ.2) GOTO 906

IF (KK.EQ.5.OR.KK.EQ.8) GOTO 906

$\mathrm{BTU}(\mathrm{II}, \mathrm{JJ}, \mathrm{KK})=0.0$

906

CONTINUE

905 CONTINUE

904

CONTINUE

DO 907 III $=3,9,3$

$\operatorname{ETAC}(I I I, I I I-2)=D 7$

$\operatorname{ETAC}($ III, III-1) $=$ D8

$\operatorname{ETAC}($ III, III $)=D 9$

DO $908 \mathrm{JJJ}=1,3$

DO $909 \mathrm{KKK}=1,8$

IF (KKK.EQ.3.OR.KKK.EQ.6) GOTO 909 
$\mathrm{BTU}(\mathrm{III}, \mathrm{JJJ}, \mathrm{KKK})=0.0$

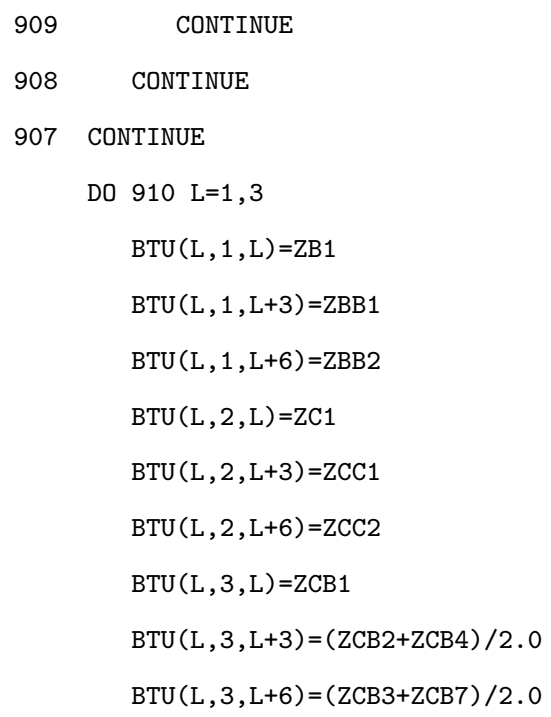

910 CONTINUE

DO $911 \mathrm{~K}=4,6$

$\operatorname{BTU}(K, 1, K-3)=Z B B 1$

$\mathrm{BTU}(\mathrm{K}, 1, \mathrm{~K})=\mathrm{ZB} 2$

$\mathrm{BTU}(\mathrm{K}, 1, \mathrm{~K}+3)=\mathrm{ZBB} 3$

$\operatorname{BTU}(K, 2, K-3)=Z C C 1$

$\operatorname{BTU}(K, 2, K)=Z C 2$

$\mathrm{BTU}(\mathrm{K}, 2, \mathrm{~K}+3)=\mathrm{ZCC} 3$

$\operatorname{BTU}(K, 3, K-3)=(Z C B 4+Z C B 2) / 2.0$

$\operatorname{BTU}(K, 3, K)=Z C B 5$

$\operatorname{BTU}(K, 3, K+3)=(\mathrm{ZCB} 6+\mathrm{ZCB} 8) / 2.0$

911 CONTINUE

DO $912 \mathrm{~N}=7,9$

$\operatorname{BTU}(\mathrm{N}, 1, \mathrm{~N}-6)=\mathrm{ZBB} 2$

$\mathrm{BTU}(\mathrm{N}, 1, \mathrm{~N}-3)=\mathrm{ZBB} 3$

$\operatorname{BTU}(N, 1, N)=Z B 3$

$\mathrm{BTU}(\mathrm{N}, 2, \mathrm{~N}-6)=\mathrm{ZCC} 2$

$\mathrm{BTU}(\mathrm{N}, 2, \mathrm{~N}-3)=\mathrm{ZCC} 3$

$\mathrm{BTU}(\mathrm{N}, 2, \mathrm{~N})=\mathrm{ZC} 3$

$\operatorname{BTU}(\mathrm{N}, 3, \mathrm{~N}-6)=(\mathrm{ZCB} 7+\mathrm{ZCB} 3) / 2.0$

$\mathrm{BTU}(\mathrm{N}, 3, \mathrm{~N}-3)=(\mathrm{ZCB} 8+\mathrm{ZCB} 6) / 2.0$

$\operatorname{BTU}(\mathrm{N}, 3, \mathrm{~N})=\mathrm{ZCB} 9$

912 CONTINUE

D0 $610 \mathrm{I}=1,9$

DO $610 \mathrm{~J}=1,9$

$\operatorname{ETACT}(J, I)=\operatorname{ETAC}(I, J)$

610 CONTINUE

DO $660 \mathrm{~K}=1,9$

$\mathrm{QK} 3(\mathrm{~K}, 1)=0.0$ 


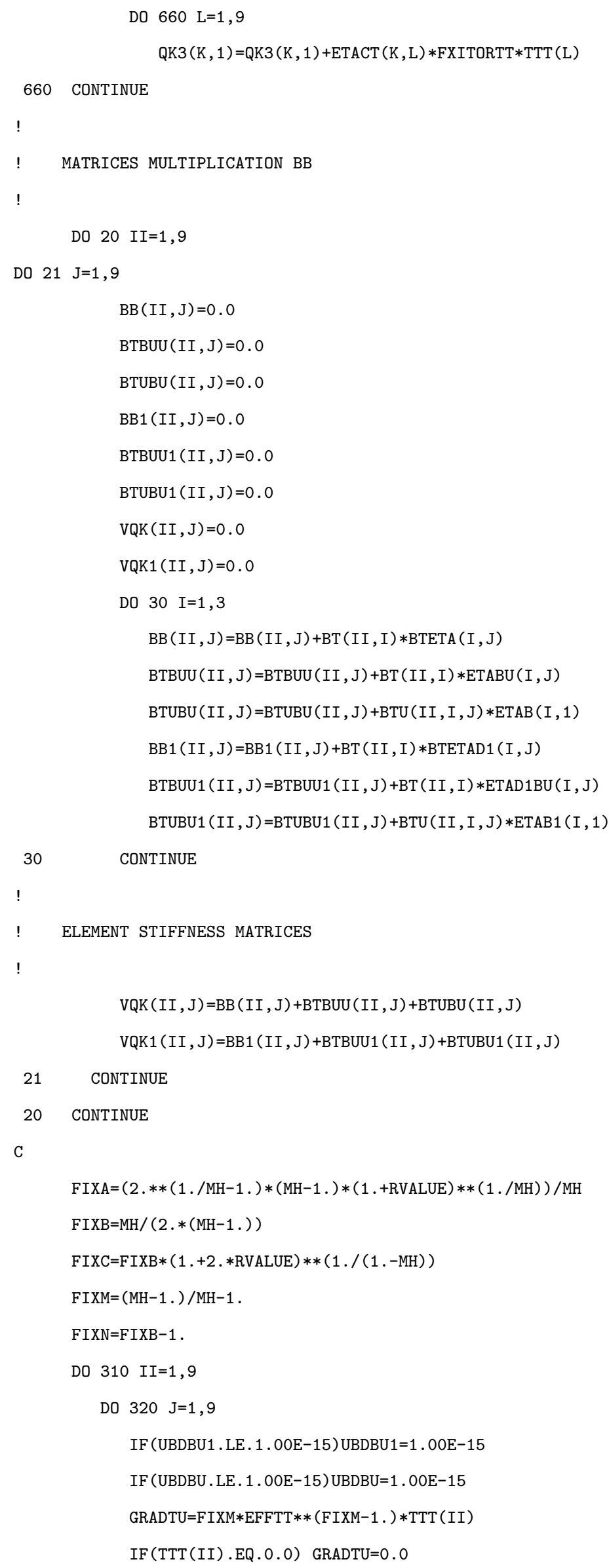


IF (EFFTT . LT . 1.00E-15) GRADTU $=0.0$

GRADT1 $=$ FIXN $*$ UBDBU1 $* *($ FIXN -1.$) * 2 . *$ BTETAD1U $(I I, 1)$

GRADT2 $=$ FIXN $*$ UBDBU $* *($ FIXN-1 . $) * 2 . * \operatorname{BTETAU}($ II, 1$)$

C

IF (FIXN.EQ . 0.0) GRADT1 $=0.0$

IF (FIXN.EQ . 0.0) GRADT2=0.0

IF (EFFTT . LE . 1.00E-15) EFFTT=1 .00E-15

FIX5 $=$ UBDBU $1 * * F I X N$

PROD1 $=($ GRADTU $*$ UBDBU1 $* *$ FIXN+EFFTT $* * F I X M * G R A D T 1) * 2$.

$\$$

*BTETAD1U $(\mathrm{J}, 1)$

PROD2 $=($ GRADTU $* U B D B U * * F I X N+E F F T T * * F I X M * G R A D T 2) * 2 . * \operatorname{BTETAU}(J, 1)$

GRADVAREFF $=$ FIXB $*($ PROD $1+E F F T T * * F I X M * U B D B U 1 * * F I X N * 2$.

*VQK1 $(\mathrm{II}, \mathrm{J}))+\mathrm{FIXC} *(\mathrm{PROD} 2+\mathrm{EFFTT} * * \mathrm{FIXM} * \mathrm{UBDBU} * * \mathrm{FIXN} * 2$.

$\mathrm{A} \quad * \operatorname{VQK}(\mathrm{II}, \mathrm{J}))$

C

PRODTT $=($ FIXA $*$ EFFTT $* * F I X M) *($ FIXA $*$ EFFTT $* *$ FIXM $) *$ TTT $($ II $) *$ TTT $(\mathrm{J})$

$\mathrm{QK}(\mathrm{II}, \mathrm{J})=\mathrm{FIXA} * \mathrm{ESO} * \mathrm{GRADVAREFF}+\mathrm{BH} *(\mathrm{PRODTT}+\mathrm{EFSTRN}(\mathrm{M}) *$

FIXA*GRADVAREFF)

IF (ICLASS . GE . 5. AND . EFSTRN (M) . LE . RIGID) QK (II , J ) $=($ ESO/RIGID

$+\mathrm{BH}) *(\mathrm{PRODTT}+\mathrm{EFSTRN}(\mathrm{M}) * \mathrm{FIXA} *$ GRADVAREFF $)$

C

$320 \quad$ CONTINUE

310 CONTINUE

C

DO $350 \quad I=1,9$

DO $350 \mathrm{~J}=1,9$

$\mathrm{QK}(\mathrm{I}, \mathrm{J})=\mathrm{QK}(\mathrm{I}, \mathrm{J}) * \mathrm{VOLU}$

350 CONTINUE

DO $620 \mathrm{I}=1,9$

D0 $620 \mathrm{~J}=1,9$

$\mathrm{QK} 1(\mathrm{I}, \mathrm{J})=0.0$

DO $630 \mathrm{~K}=1,9$

$\mathrm{QK} 1(\mathrm{I}, \mathrm{J})=\mathrm{QK} 1(\mathrm{I}, \mathrm{J})+\mathrm{QK}(\mathrm{I}, \mathrm{K}) * \operatorname{ETAC}(\mathrm{K}, \mathrm{J})$

630

CONTINUE

620 CONTINUE

D0 $640 \mathrm{I}=1,9$

DO $640 \mathrm{~J}=1,9$

$\mathrm{QK} 2(\mathrm{I}, \mathrm{J})=0.0$

DO $650 \mathrm{~K}=1,9$

$\mathrm{QK} 2(\mathrm{I}, \mathrm{J})=\mathrm{QK} 2(\mathrm{I}, \mathrm{J})+\operatorname{ETACT}(\mathrm{I}, \mathrm{K}) * \mathrm{QK} 1(\mathrm{~K}, \mathrm{~J})$

650

CONTINUE

640 CONTINUE 
! OBTAIN THE PRODUCT OF BBU AND CALCULATE THE GLOBAL LOAD VECTOR

DO $40 \mathrm{KK}=1,9$

$\operatorname{BTETAU}(K K, 1)=\mathrm{QK} 3(\mathrm{KK}, 1)$

$\mathrm{HH}(\mathrm{KK})=\mathrm{BTETAU}(\mathrm{KK}, 1) * \mathrm{VOLU}$

$Q(K K)=-H H(K K)$

40 CONTINUE

!

$\operatorname{LIM}=9$

DO 70 I=3,LIM, 3

I $\mathrm{J}=\mathrm{I} / 3$

$\mathrm{LP}(\mathrm{I}-2)=3 * \mathrm{IE}(\mathrm{IJ}, \mathrm{M})-2$

$\operatorname{LP}(\mathrm{I}-1)=3 * \operatorname{IE}(\mathrm{IJ}, \mathrm{M})-1$

$70 \quad \mathrm{LP}(\mathrm{I})=3 * \operatorname{IE}(\mathrm{IJ}, \mathrm{M})$

DO $80 \mathrm{~J}=1$,LIM

$\mathrm{MO}=\mathrm{LP}(\mathrm{J})$

$R(M O)=R(M O)+Q(J)$

DO $90 \mathrm{~K}=1$, LIM

$\mathrm{NO}=\mathrm{LP}(\mathrm{K})$

IF (NO.LE.O) GOTO 90

$\mathrm{QK}(\mathrm{J}, \mathrm{K})=\mathrm{QK} 2(\mathrm{~J}, \mathrm{~K})$

$A K(M O, N O)=A K(M O, N O)+Q K(J, K)$

90 CONTINUE

80 CONTINUE

60 CONTINUE

! STORE THE OVERALL STIFFNESS MATRIX AND LOAD VECTOR

DO $700 \mathrm{MO}=1$, NEQ

$\mathrm{HH}(\mathrm{MO})=-\mathrm{R}(\mathrm{MO})$

DO 700 NO=1, NEQ

$\operatorname{SAVES}(M O, N O)=A K(M O, N O)$

700 CONTINUE

916 RETURN

END 


\section{A.2 Sub-rotina STRAIN}

SUBROUTINE STRAIN (M, LL , MM, NN , X , Y , U , V , W , DELTA, TO , DT , ESO , BH , EFSTRN,

1 TT, EFO , EX , EY , EXY , TAUO , EFF , DEF , EXZ, EYZ, EZ , EFSTRO, EFSTRT , TSTRAN

2 ,DEFO, DTO, TSTRAO)

$!$

THIS SUBROUTINE CALCULATE THE ELEMENT STRAIN AND STRESS

$!$

DIMENSION X(1), Y(1),U(1),V(1),W(1), EFSTRN (1), EX (1), EY (1), EXY(1),

$\$ \quad \operatorname{TAUO}(1), \operatorname{DEF}(1), \operatorname{EXZ}(1), \operatorname{EYZ}(1), \operatorname{EZ}(1), \operatorname{EFO}(1), \operatorname{EFF}(1), \operatorname{EFSTRO}(1)$,

2

EFSTRT (1), $\operatorname{TSTRAN}(1), \operatorname{DEFO}(1), \operatorname{TSTRAO}(1)$

COMMON/ONE/ MAXNP, MAXEL, MAXMAT, ICLASS

COMMON/TWO/ NNP, NEL, NEQ, SIGO(1)

COMMON/FIVE/ THC, FMDI , XN , RA, WIDTH, XENGTH , FM

COMMON/CMAT/ CONT1, CONT2, RVALUE

COMMON/HILL79/ MH

COMMON/VISCO/ ISRE

COMMON/GRAD/ $\operatorname{DFGRD~}(400,9), \operatorname{DJACOB}(400)$

COMMON/THICK/ THCKC (400), TUP (400)

REAL MH

C

$\mathrm{B} 1=\mathrm{Y}(\mathrm{MM})-\mathrm{Y}(\mathrm{NN})$

$B 2=Y(N N)-Y(L L)$

$B 3=Y(L L)-Y(M M)$

$\mathrm{C} 1=\mathrm{X}(\mathrm{NN})-\mathrm{X}(\mathrm{MM})$

$\mathrm{C} 2=\mathrm{X}(\mathrm{LL})-\mathrm{X}(\mathrm{NN})$

$\mathrm{C} 3=\mathrm{X}(\mathrm{MM})-\mathrm{X}(\mathrm{LL})$

DELTA $=\mathrm{X}(\mathrm{MM}) * \mathrm{Y}(\mathrm{NN})$

IF (DELTA.LE.0.0) GOTO 10

$\mathrm{XX}=$ DELTA

$\operatorname{DUDX}=(\mathrm{B} 1 * \mathrm{U}(\mathrm{LL})+\mathrm{B} 2 * \mathrm{U}(\mathrm{MM})+\mathrm{B} 3 * \mathrm{U}(\mathrm{NN})) / \mathrm{XX}$

DUDY $=(\mathrm{C} 1 * \mathrm{U}(\mathrm{LL})+\mathrm{C} 2 * \mathrm{U}(\mathrm{MM})+\mathrm{C} 3 * \mathrm{U}(\mathrm{NN})) / \mathrm{XX}$

$\mathrm{DVDX}=(\mathrm{B} 1 * \mathrm{~V}(\mathrm{LL})+\mathrm{B} 2 * \mathrm{~V}(\mathrm{MM})+\mathrm{B} 3 * \mathrm{~V}(\mathrm{NN})) / \mathrm{XX}$

$\mathrm{DVDY}=(\mathrm{C} 1 * \mathrm{~V}(\mathrm{LL})+\mathrm{C} 2 * \mathrm{~V}(\mathrm{MM})+\mathrm{C} 3 * \mathrm{~V}(\mathrm{NN})) / \mathrm{XX}$

$\mathrm{DWDX}=(\mathrm{B} 1 * \mathrm{~W}(\mathrm{LL})+\mathrm{B} 2 * \mathrm{~W}(\mathrm{MM})+\mathrm{B} 3 * \mathrm{~W}(\mathrm{NN})) / \mathrm{XX}$

$\mathrm{DWDY}=(\mathrm{C} 1 * \mathrm{~W}(\mathrm{LL})+\mathrm{C} 2 * \mathrm{~W}(\mathrm{MM})+\mathrm{C} 3 * \mathrm{~W}(\mathrm{NN})) / \mathrm{XX}$ 
! COMPUTATIONS OF DEFORMATION GRADIENTS

!

$\operatorname{DFGRD}(\mathrm{M}, 1)=1.0+\operatorname{DUDX}$

$\operatorname{DFGRD}(\mathrm{M}, 2)=\operatorname{DUDY}$

$\operatorname{DFGRD}(M, 3)=0.0$

$\operatorname{DFGRD}(M, 4)=\operatorname{DVDX}$

$\operatorname{DFGRD}(M, 5)=1.0+\operatorname{DVDY}$

$\operatorname{DFGRD}(M, 6)=0.0$

$\operatorname{DFGRD}(M, 7)=\operatorname{DWDX}$

$\operatorname{DFGRD}(\mathrm{M}, 8)=\operatorname{DWDY}$

$\operatorname{DFGRD}(M, 9)=1.0$

$!$

! DETERMINATION OF JACOBIANS

$\operatorname{DJACOB}(M)=\operatorname{DFGRD}(M, 1) * \operatorname{DFGRD}(M, 5)-\operatorname{DFGRD}(M, 2) * \operatorname{DFGRD}(M, 4)$

!

$\mathrm{EX}(\mathrm{M})=\mathrm{DUDX}+0.5 *(\mathrm{DUDX} * * 2+\mathrm{DVDX} * * 2+\mathrm{DWDX} * * 2)$

$\mathrm{EY}(\mathrm{M})=\mathrm{DVDY}+0.5 *(\mathrm{DUDY} * * 2+\mathrm{DVDY} * * 2+\mathrm{DWDY} * * 2)$

$\operatorname{EXY}(\mathrm{M})=0.5 *(\mathrm{DUDY}+\mathrm{DVDX}+\mathrm{DUDX} * \mathrm{DUDY}+\mathrm{DVDX} * \mathrm{DVDY}+\mathrm{DWDX} * \mathrm{DWDY})$

$\operatorname{EXZ}(M)=0.5 * \operatorname{DWDX}$

$\operatorname{EYZ}(\mathrm{M})=0.5 * \mathrm{DWDY}$

$\operatorname{EXZ}(\mathrm{M})=2 \cdot 0 * \operatorname{EXZ}(\mathrm{M})$

$\operatorname{EYZ}(\mathrm{M})=2 \cdot 0 * \mathrm{EYZ}(\mathrm{M})$

$\operatorname{EXZ}(M)=0.0$

$\operatorname{EYZ}(\mathrm{M})=0.0$

$E Z(M)=-E X(M)-E Y(M)$

!

! TO CALCULATE THE ELEMENT EFFECTIVE STRESSES AND STRAINS

C HILL 79

C

$\mathrm{CR}=2 . * *(-1 .+1 . / \mathrm{MH}) *(1 .+\mathrm{RVALUE}) * *(1 . / \mathrm{MH})$

$\mathrm{SS} 1=((\mathrm{EX}(\mathrm{M})+\mathrm{EY}(\mathrm{M})) * * 2) * *(\mathrm{MH} /(2 * *(-1 .+\mathrm{MH})))$

$\mathrm{SS} 2=(4 . * \mathrm{EXY}(\mathrm{M}) * \mathrm{EXY}(\mathrm{M})+(\mathrm{EX}(\mathrm{M})-\mathrm{EY}(\mathrm{M})) * * 2) * *(\mathrm{MH} /(2 \cdot *(-1 .+\mathrm{MH})))$

$\operatorname{EFSTRN}(\mathrm{M})=\mathrm{CR} *(\mathrm{SS} 1+\mathrm{SS} 2 *(1 .+2 . * \mathrm{RVALUE}) * *(1 . /(1 .-\mathrm{MH}))) * *((-1 .+\mathrm{MH}) / \mathrm{MH})$

$\mathrm{TT}=\mathrm{TO}+\mathrm{DT}$

$\operatorname{DEF}(\mathrm{M})=0.5 * \operatorname{ALOG}(1 \cdot 0+2 \cdot 0 * \operatorname{EFSTRN}(\mathrm{M}))$

$\mathrm{EFF}(\mathrm{M})=\mathrm{EFO}(\mathrm{M})+\mathrm{DEF}(\mathrm{M})$

$!$ RIGID-PLASTIC CONSTITUTIVE EQUATION 
$!$

$\mathrm{ESO}=\operatorname{CONT} 1 *(\operatorname{CONT} 2+\mathrm{EFO}(\mathrm{M})) * * \mathrm{XN}$

$\mathrm{SH}=\mathrm{XN} * \mathrm{CONT} 1 *(\mathrm{CONT} 2+\mathrm{EFO}(\mathrm{M})) * *(\mathrm{XN}-1.0)$

IF (ICLASS.NE.4) GOTO 50

$\operatorname{IF}(E F O(M) . G T .0 .36)$ GOTO 41

$\mathrm{ESO}=\mathrm{CONT} 1 *(\mathrm{CONT} 2+0.422569 * \mathrm{EFO}(\mathrm{M}) * * \mathrm{XN})$

$\mathrm{SH}=\mathrm{CONT} 1 * \mathrm{XN} * 0.422563 * \mathrm{EFO}(\mathrm{M}) * *(\mathrm{XN}-1.0)$

GOTO 50

$41 \mathrm{ESO}=\mathrm{CONT} 1 *(\mathrm{CONT} 2+0.37089 * \mathrm{EFO}(\mathrm{M}) * * 0.375)$

$\mathrm{SH}=\mathrm{CONT} 1 * 0.375 * 0.37089 * \mathrm{EFO}(\mathrm{M}) * *(-0.625)$

$50 \mathrm{BH}=\mathrm{SH}-2.0 * \mathrm{ESO}$

GOTO 60

$!$

$60 \operatorname{EXY}(\mathrm{M})=2.0 * \operatorname{EXY}(\mathrm{M})$

$!$

THICKNESS STRAIN CALCULATION

$\operatorname{THCKC}(\mathrm{M})=\operatorname{TUP}(\mathrm{M}) *(1.0+\mathrm{EZ}(\mathrm{M}))$

$\operatorname{EFSTRT}(M)=A L O G(\operatorname{THCKC}(M) / T H C)$

$\operatorname{TSTRAN}(M)=\operatorname{TSTRAO}(M)+\operatorname{EFSTRN}(M)$

$\operatorname{TAUO}(\mathrm{M})=\mathrm{ESO}+\mathrm{BH} * \mathrm{EFSTRN}(\mathrm{M})$

IF (DELTA.GT.0.0) GOTO 30

10 WRITE $(16,20)$ M, DELTA

20 FORMAT(//5X, 'THE AREA OF TRIANGLE NO.', I8,5X,'IS $\ldots . .$. ', F12.5)

30 RETURN

END 


\section{ANEXOB - Obtenção de $\mathrm{P}^{*}$}

A equação 5.56, matriz de rigidez global $\mathbf{P}^{*}$, é obtida a partir da equação

$$
\mathbf{P}^{*}=\left(\frac{\mathrm{d}}{\mathrm{d} \mathbf{u}}\left\{\int_{V} \bar{S} \delta(\mathrm{d} \bar{E}) \mathrm{d} V-\int_{S} \delta \mathbf{u} \mathbf{N}^{T} \mathbf{f} \mathrm{d} S\right\} \frac{\mathrm{d} \mathbf{u}}{\mathrm{d} \mathbf{U}}\right)^{*}
$$

como (ver seção 3.7)

$$
\bar{S}=\bar{\sigma}_{0}+\left(h_{0}-2 \bar{\sigma}_{0}\right) \mathrm{d} \bar{E}
$$

a equação B.1 pode ser reescrita

$$
\mathbf{P}^{*}=\left(\frac{\mathrm{d}}{\mathrm{d} \mathbf{u}}\left\{\int_{V}\left(\bar{\sigma}_{0}+\left(h_{0}-2 \bar{\sigma}_{0}\right) \mathrm{d} \bar{E}\right) \delta(\mathrm{d} \bar{E}) \mathrm{d} V-\int_{S} \delta \mathbf{u} \mathbf{N}^{T} \mathbf{f} \mathrm{d} S\right\} \frac{\mathrm{d} \mathbf{u}}{\mathrm{d} \mathbf{U}}\right)^{*}
$$

então tem-se

$$
\mathbf{P}^{*}=\left(\frac{\mathrm{d}}{\mathrm{d} \mathbf{u}}\left\{\int_{V} \bar{\sigma}_{0} \delta(\mathrm{d} \bar{E}) \mathrm{d} V+\int_{V}\left(h_{0}-2 \bar{\sigma}_{0}\right) \mathrm{d} \bar{E} \delta(\mathrm{d} \bar{E}) \mathrm{d} V-\int_{S} \delta \mathbf{u} \mathbf{N}^{T} \mathbf{f} \mathrm{d} S\right\} \frac{\mathrm{d} \mathbf{u}}{\mathrm{d} \mathbf{U}}\right)^{*}
$$

com

$$
\frac{\mathrm{d} \mathbf{u}}{\mathrm{d} \mathbf{U}}=\Lambda
$$

Sendo $\mathrm{d} \bar{E}$ um escalar e $\delta(\mathrm{d} \bar{E})$ um tensor de primeira ordem o segundo termo da equação B.4 é obtido da seguinte forma

$$
\frac{\mathrm{d}}{\mathrm{d} \mathbf{u}}\{\mathrm{d} \bar{E} \delta(\mathrm{d} \bar{E})\}=\mathrm{d} \bar{E} \frac{\mathrm{d}}{\mathrm{d} \mathbf{u}}\{\delta(\mathrm{d} \bar{E})\}+\delta(\mathrm{d} \bar{E}) \otimes \frac{\mathrm{d}}{\mathrm{du}}\{\mathrm{d} \bar{E}\}
$$

com o símbolo $\otimes$ representando o produto tensorial. 